\title{
Predicted Impacts of Future Water Level Decline on Monitoring Wells Using a Ground-Water Model of the Hanford Site
}

S. K. Wurstner

M. D. Freshley

December 1994

Prepared for the U.S. Department of Energy under Contract DE-AC06-76RLO 1830

Pacific Northwest Laboratory

Richland, Washington 99352

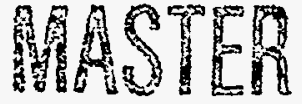




\section{DISCLAIMER}

This report was prepared as an account of work sponsored by an agency of the United States Government. Neither the United States Government nor any agency thereof, nor any of their employees, make any warranty, express or implied, or assumes any legal liability or responsibility for the accuracy, completeness, or usefulness of any information, apparatus, product, or process disclosed, or represents that its use would not infringe privately owned rights. Reference herein to any specific commercial product, process, or service by trade name, trademark, manufacturer, or otherwise does not necessarily constitute or imply its endorsement, recommendation, or favoring by the United States Government or any agency thereof. The views and opinions of authors expressed herein do not necessarily state or reflect those of the United States Government or any agency thereof. 


\section{DISCLAIMER}

Portions of this document may be illegible in electronic image products. Images are produced from the best available original document. 


\section{Executive Summary}

Since Hanford Site operations were curtailed in 1987, and the Site mission has shifted from the production of nuclear materials to environmental restoration, waste management, and technology development, the volume of water discharged to the ground has been greatly reduced. As a result, the water table has begun to decline, potentially impacting existing monitoring wells used by contractors on the Hanford Site. Pacific Northwest Laboratory conducted a study for the U.S. Department of Energy, as part of the Hanford Site Ground-Water Surveillance Project, to evaluate the impacts of declining water levels on existing monitoring wells in the unconfined aquifer system at the Hanford Site.

A ground-water flow model was used to predict water level decline in selected wells in the operating areas $(100,200,300$, and 400 Areas) and the 600 Area. To predict future water levels, the unconfined aquifer system was simulated with the two-dimensional version of a ground-water model of the Hanford Site, which is based on the Coupled Fluid, Energy, and Solute Transport (CFEST) Code in conjunction with the Geographic Information Systems (GIS) software package. The model was developed using the assumption that artificial recharge to the unconfined aquifer system from Site operations was much greater than any natural recharge from precipitation or from the basalt aquifers below. However, artificial recharge is presently decreasing and projected to decrease even more in the future. As the flow system approaches pre-Hanford conditions, this assumption will become invalid and it will be crucial to include natural recharge in the model to accurately represent the water balance and the driving forces that control water movement.

Wells currently used for monitoring at the Hanford Site are beginning to go dry or are difficult to sample, and as the water table declines over the next 5 to 10 years, a larger number of wells is expected to be impacted. The water levels predicted by the ground-water model were compared with monitoring well completion intervals to determine which wells will become dry in the future. Predictions of wells that will go dry within the next 5 years have less uncertainty than predictions for wells that will become dry within 5 to 10 years. Each prediction is an estimate based on assumed future Hanford Site operating conditions and model assumptions. Future conditions that differ from those used in the model will result in a different hydrologic response. 
Dewatering of the unconfined aquifer in response to decreased wastewater discharge in the future will be transient, and portions of the aquifer will release water at different times. The effects of declining discharges at the Hanford Site will be observed in the unconfined aquifer system for several decades to come. 


\section{Acknowledgments}

Several people contributed to the development of this report. The authors would like to thank John McDonald for generating the hydrographs and compiling the effluent discharge data for the model inputs. The following people provided information on effluent volumes and projections for future Site operations: Sandy Thomas, Westinghouse Hanford Company (WHC); Scott Meyer, City of Richland; Marvin McCarthy, WHC; Jim Chasse, Washington Public Power Supply System (WPPSS); Kristi Lueck, WHC; Craig Perkins, WHC; A. J. (Tony) Diliberto, WHC; Michael Brown, WHC; and Joseph Thrasher, WHC. Mickie Chamness compiled the well screen data, and Bill Webber provided selected results from his letter report for inclusion in this study. Thanks and appreciation also go to Charlie Cole, Evan Dresel, and Stuart Luttrell for providing technical peer reviews. 


\section{Contents}

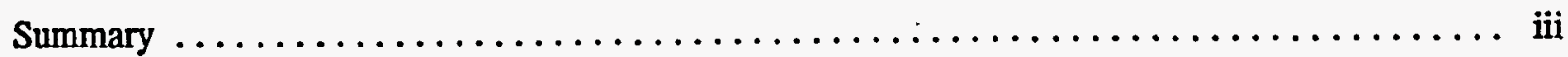

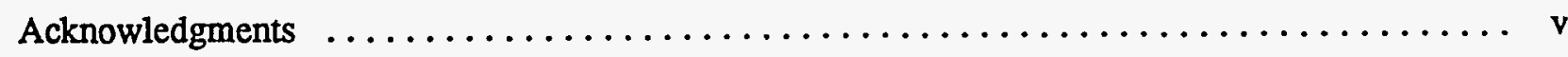

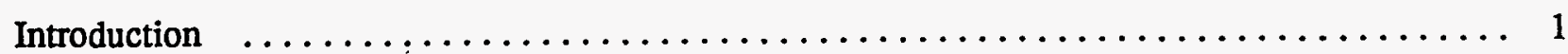

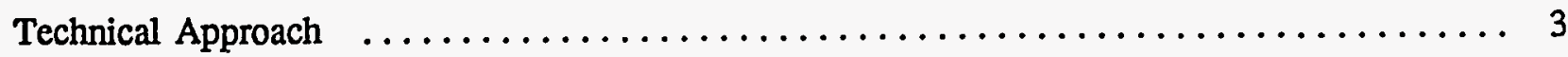

Assumptions and Limitations $\ldots \ldots \ldots \ldots \ldots \ldots \ldots \ldots \ldots \ldots \ldots \ldots \ldots \ldots \ldots \ldots \ldots \ldots \ldots$

Results and Discussion $\ldots \ldots \ldots \ldots \ldots \ldots \ldots \ldots \ldots \ldots \ldots \ldots \ldots \ldots \ldots \ldots \ldots \ldots \ldots \ldots \ldots \ldots \ldots, 13$

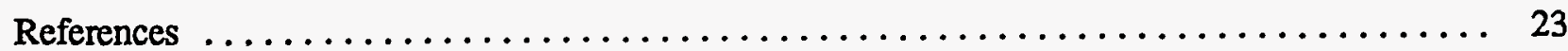

Appendix A - Estimates of Effluent Discharge Volumes for the Hanford Site $\ldots \ldots \ldots \ldots$ A.1

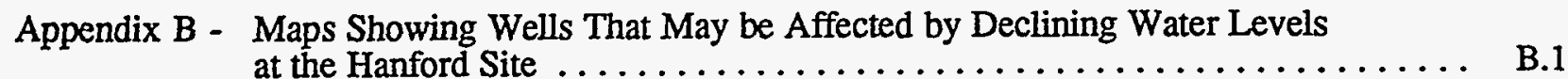

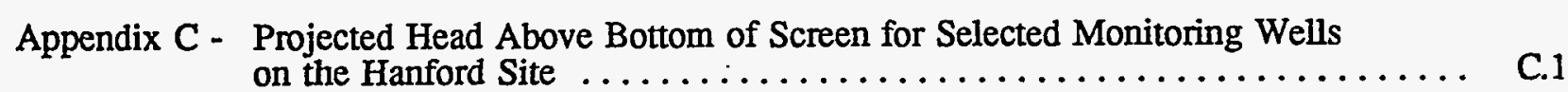

Appendix D - $\begin{aligned} & \text { Hydrographs of Selected Monitoring Wells Showing Observed Data and } \\ & \text { Model Results } \ldots \ldots \ldots \ldots \ldots \ldots \ldots \ldots \ldots \ldots \ldots \ldots \ldots \ldots \ldots \ldots \ldots \ldots \ldots \ldots \ldots\end{aligned}$ 


\section{Figures}

1. Map of Wells Used in this Study $\ldots \ldots \ldots \ldots \ldots \ldots \ldots \ldots \ldots \ldots \ldots \ldots$

2. Hanford Effluent Discharge Sites Included in the Model $\ldots \ldots \ldots \ldots \ldots \ldots \ldots$

3. Bar Graph of Waste Streams in the 200 East, 200 West, 100-BC, and 100-N Areas of the Hanford Site, 1979 through 1993

4. Comparison of Model Results to Interpreted Water Table Contours for

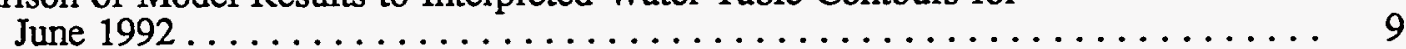

5. Model Results for December $1995 \ldots \ldots \ldots \ldots \ldots \ldots \ldots \ldots \ldots \ldots \ldots \ldots \ldots$

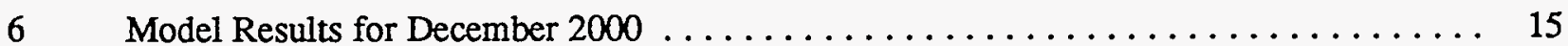

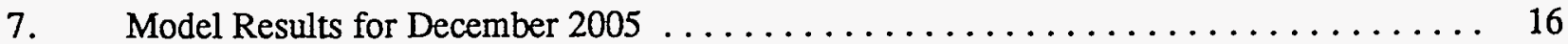

8. Modeled Water Table Changes From December 1993 to December 2005 .................................. 17

9. Predicted Impact of Declining Water Levels on 200 East Area Wells for January 2000, Determined Using the Ground Water Model . . . . . . . . . . . . 18

10. Predicted Impact of Declining Water Levels on 200 East Area Wells for January 2000, Determined Using the Straight-Line Projection Method . . . . . . . . 19

11. Predicted Impact of Declining Water Levels on 200 West Area Wells for January 2000 , Determined Using the Ground Water Model .............. 20

12. Predicted Impact of Declining Water Levels on 200 West Area Wells for January 2000, Determined Using the Straight-Line Projection Method

\section{Table}

1. Specific-Yield Data from the Hanford Site 


\section{Introduction}

The purpose of this study was to predict the impacts of declining water levels on monitoring wells in the unconfined aquifer system at the Hanford Site. The scope of this effort includes evaluation of monitoring wells in the operating areas $(100,200,300$, and 400 Areas) and across the Site in the 600 Area.

Discharges of wastewater to the ground at the Hanford Site have created ground-water mounds in the unconfined aquifer system near each of the major operating areas (Woodruff et al. 1993). Water levels have changed continually during Site operations because of variations in the volume of wastewater discharged (Zimmerman et al. 1986; Newcomer 1990). Since 1987, the Site mission has shifted from the production of nuclear materials to environmental restoration, waste management, and technology development. To restrict further degradation of the ground water, DOE and the Washington Department of Ecology have signed Consent Order Number 91NM-177. Under this order, disposal of untreated effluent to the soil column will be discontinued after June 1995. The Consent Order identifies Phase I and II streams. Tri-Party Agreement (TPA) Milestone M-17-00 "Complete Liquid Effluent Treatment Facilities/Upgrades for all Phase I Streams" requires DOE to cease disposal of all untreated effluent to the soil column by June 1995. In response to this drastic decrease in the volume of wastewater discharged to the ground, the water table has begun to decline, potentially impacting existing monitoring wells used by contractors on the Hanford Site.

This evaluation of impacts to existing monitoring wells was conducted under the Hanford Site Ground-Water Surveillance Project for the U.S. Department of Energy. A ground-water model of the unconfined aquifer system at the Hanford Site (Wurstner and Devary 1993) based on the Coupled Fluid, Energy, and Solute Transport (CFEST) Code was used in conjunction with the commercially available Geographic Information Systems (GIS) software package, Arc/Info.

Model predictions developed for this study are compared with those of a companion study, funded by Westinghouse Hanford Company, which makes estimates on a straight-line projection of well hydrographsa.

(a) Webber, W. D., and J. P. McDonald. 1994. Impact of Declining Water Levels on Ground-Water Monitoring Networks at the Hanford Site, Richland, Washington. Letter Report for Westinghouse Hanford Company by Pacific Northwest Laboratory, Richland, Washington. 



\section{Technical Approach}

The approach used in this study was to predict water level decline in selected wells with a ground-water flow model. The future water levels were then compared with completion depths and screened intervals of wells used for monitoring the unconfined aquifer system.

To predict future water levels for the monitoring well evaluation, the aquifer was simulated with the two-dimensional model based on CFEST (Wurstner and Devary 1993). The transmissivity distribution and initial conditions were derived from the inverse calibration performed for 1979 (Jacobson and Freshley 1990). The distribution of hydraulic heads from the inverse calibration (1979 data) was used as initial conditions for the simulation. The simulation was conducted in two stages. The first stage consisted of predicting water levels through 1993 and comparing with observed maps of hydraulic heads. The second stage consisted of continuing the simulations to project the future water table response.

The hydraulic heads predicted with the ground-water flow model were compared with the bottom of screened or perforated intervals recorded for wells in the unconfined aquifer system (Chamness and Merz 1993). This comparison provides an estimate of whether the well is "dry" or how much head is available in the well for operating sample pumps. Because CFEST predicts hydraulic heads at nodes that may not coincide with well locations, Arc/Info was used to interpolate head values from CFEST to the well locations. Arc/Info was also used to generate plots of wells in categories according to the height of the water column available for sampling. All Hanford Site wells located within the model boundary, and for which screen interval information was available, were included in the study and are illustrated in Figure 1.

Monthly effluent discharges from 1980 to 1992 were based on WHC effluent reports. Figure 2 shows the location of these discharge sites, and Figure 3 summarizes and compares the aggregate volumes in the 200 East, $200 \mathrm{West}, 100-\mathrm{BC}$, and $100-\mathrm{N}$ areas. The discharge estimates were revised based on input from staff members responsible for effluent monitoring. The revised estimates, with an assessment of their quality, is provided in Appendix A. Effluent discharges for 1993 to 2005 are also based on projected Site operations and decreased wastewater discharges. These estimates are also summarized in Appendix A. No natural recharge was included in these simulations, although it is recognized to occur on the Hanford Site (Gee et al. 1992). Natural 


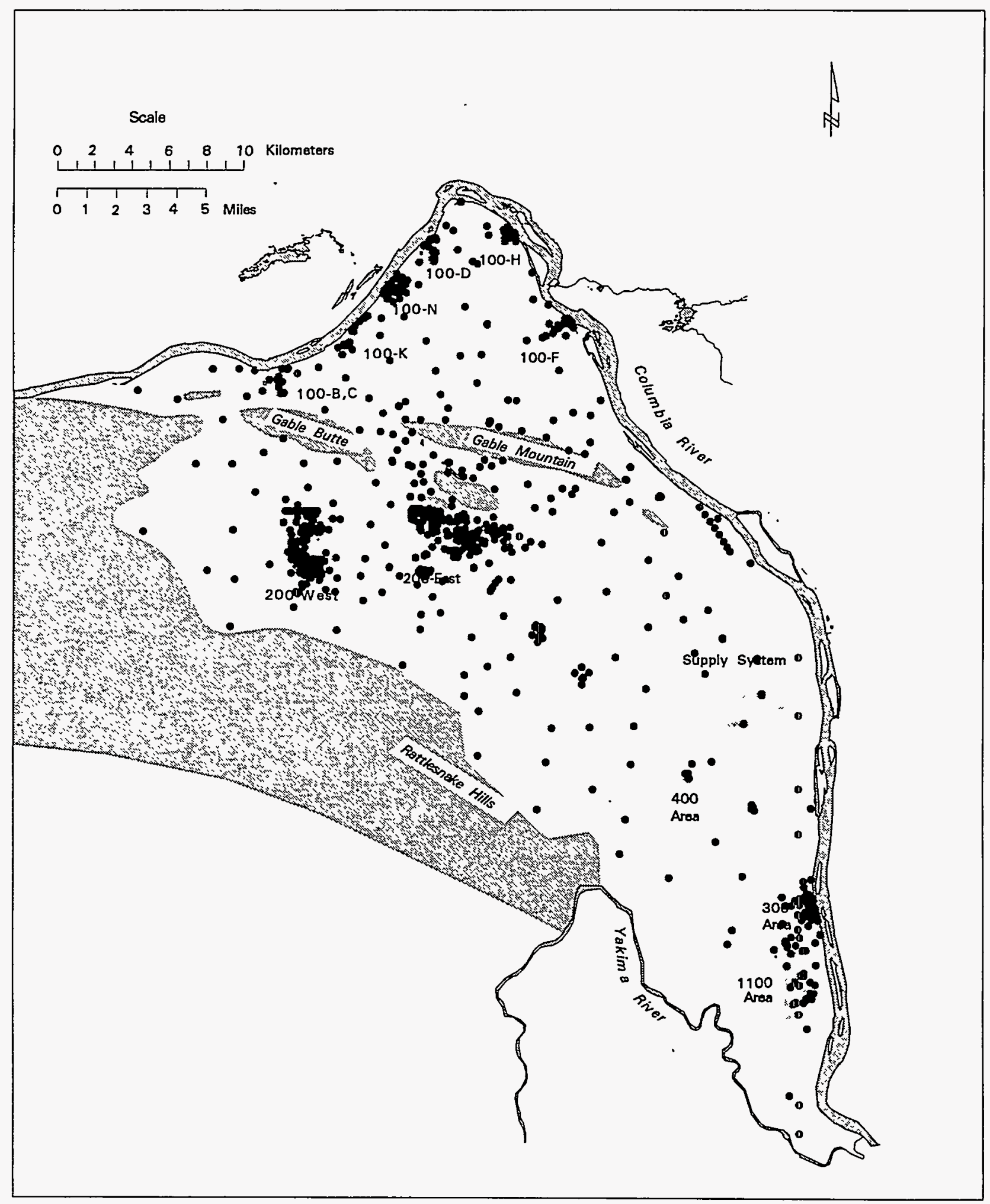

Figure 1. Map of Wells Used in this Study. 


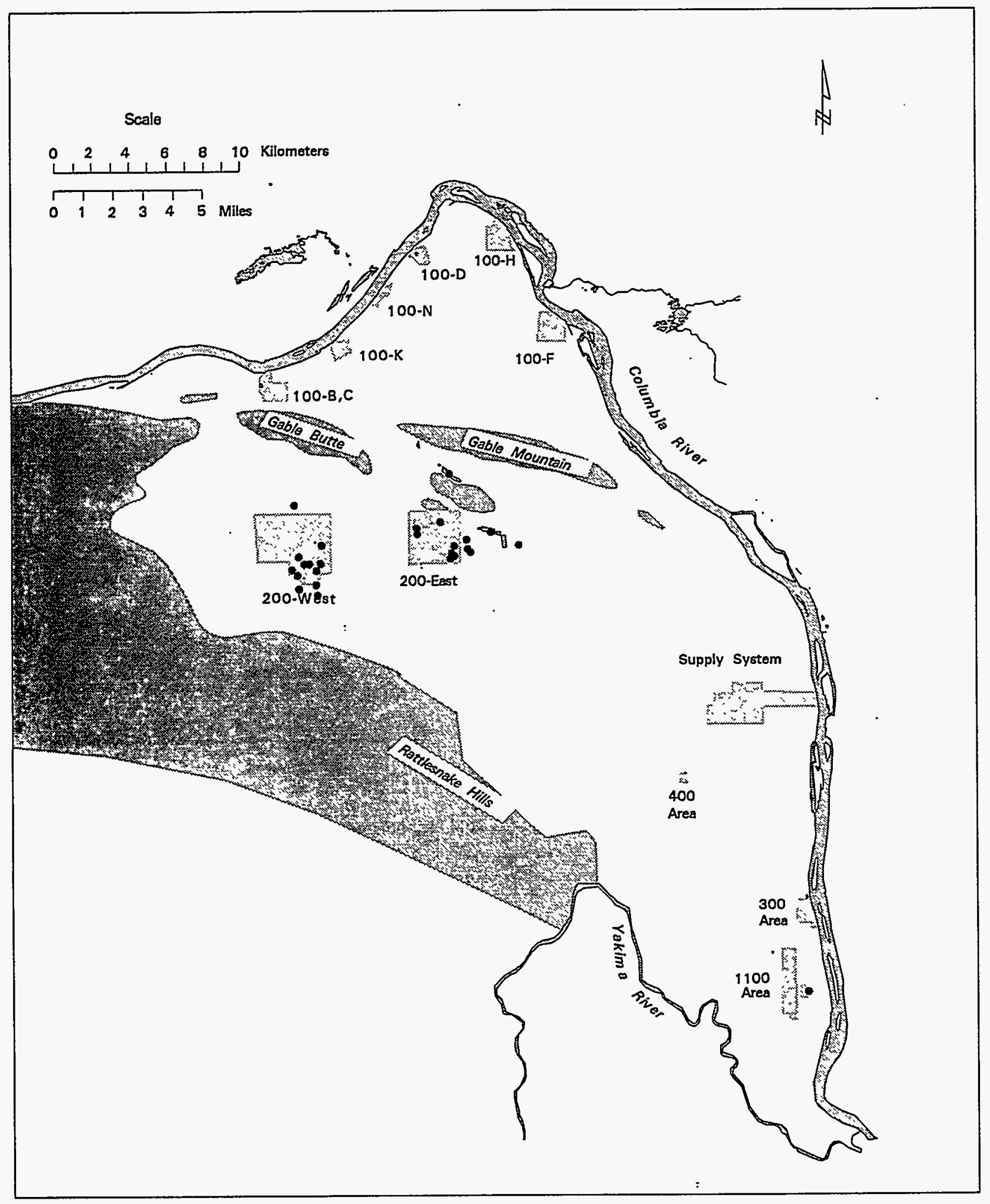

Figure 2. Hanford Effluent Discharge Sites Included in the Model. 


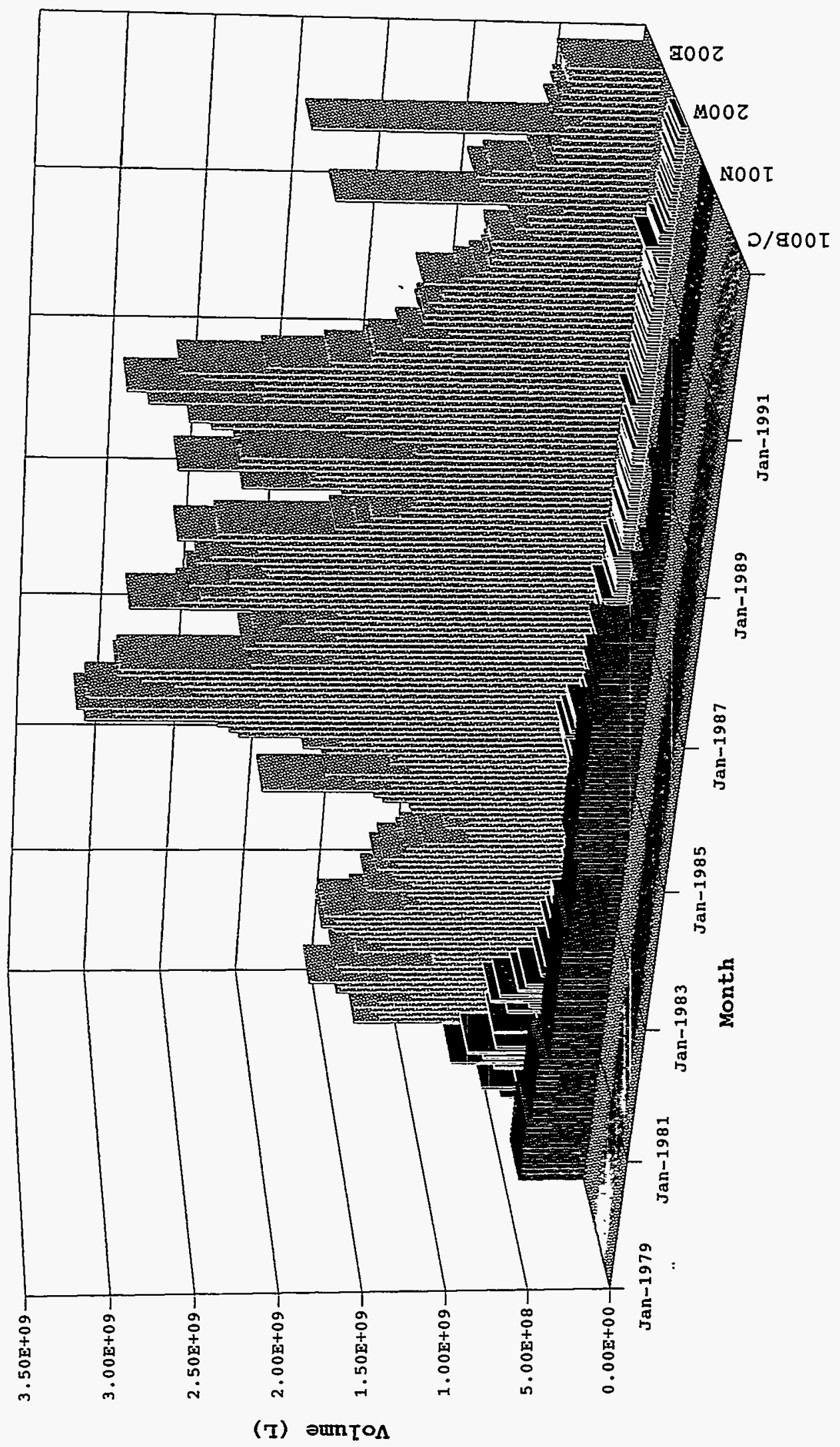

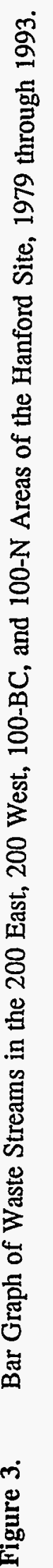


Table 1. Specific Yield Data from the Hanford Site

\begin{tabular}{lccl}
\multicolumn{1}{c}{ Well } & Storativity & Specific Yield & \multicolumn{1}{c}{ Reference } \\
\hline 199-K-10 & 0.00007 & 0.04 & Bierschenk 1957 \\
699-S22-E9B & 0.005 & 0.02 & Swanson 1992 \\
699-S27-E9A & 0.013 & 0.37 & Swanson et al. 1994 \\
699-36-61B & & 0.05 & Kipp and Mudd 1973 \\
699-37-82A & 0.02 & 0.18 & Graham et al. 1981 \\
$699-43-88$ & & 0.05 & Graham et al. 1981 \\
$699-47-35 C$ & 0.002 & 0.15 & Graham et al. 1981 \\
$699-55-50 B$ & & 0.2 & Bierschenk 1957, Thorne and \\
& & & Newcomer 1992 \\
$699-62-43 B$ & & 0.06 & Bierschenk 1957
\end{tabular}

recharge will be included in future work with the two- and three-dimensional models being developed by the Ground-Water Surveillance Project.

Boundary conditions are the same as those described in Wurstner and Devary (1993). These consist primarily of prescribed head along the Columbia and Yakima rivers, no-flow boundaries at the bottom of the aquifer and along the basalt outcrops and subcrops, and constant flux along a portion of Rattlesnake Mountain (reflecting discharges from springs) and along the Dry Creek valley. A prescribed head boundary was also used at the entrance of Cold Creek Valley onto the Hanford Site. This boundary is impacted by offsite irrigation upgradient from the Hanford Site boundary. A value of $150 \mathrm{~m}$ was used to reflect recent water level observations in well 699-43-104, located near the boundary. No natural recharge from precipitation was included and no interconnection between the unconfined aquifer and the deeper basalt aquifers was accounted for.

The transient simulation used in this study required specification of the storativity, or specific yield, as it is labeled for an unconfined aquifer. Relatively few specific yield values have been measured for the unconfined aquifer at the Hanford Site because determination of this parameter requires a multiple-well pumping test. The values that have been reported (summarized in Table 1$)$ are similar to the normal range of specific yield $(0.01$ to 0.30$)$ reported for unconfined aquifers (Freeze and Cherry 1979). 
The approach used for treating specific yield was to assume a constant value for the entire aquifer. While specific yield is recognized to vary spatially with transmissivity, the few measurements did not provide a basis to assign different values to different elements or zones. This will be investigated as part of the three-dimensional model development because it will be important for transient simulations with that model, and the effects of spatial variability between layers may be important. Several values were tried until the predicted heads for 1992 provided a good match to the interpretation of measured values (Figure 4). A specific yield of 0.35 provided the best match. As shown in Figure 4, the predicted and observed contours match in most locations on the Site, subject to interpretation of the measured and predicted values. Several areas of interpretation are different around Gable Mountain. The area north of Gable Mountain is the location of a possible zone of perched water and the interpretations south and east of Gable Mountain reflect measurements at only a few well locations. For most of the 200 East and 200 West Areas, the model is in good agreement with observations. Once the match between predicted and observed heads was judged to be adequate by visual inspection, the simulations were continued through the year 2005. The model predicted flow conditions 12 years into the future (1993 - 2005), approximately the same duration for which it had reproduced observations (1979 - 1992). 


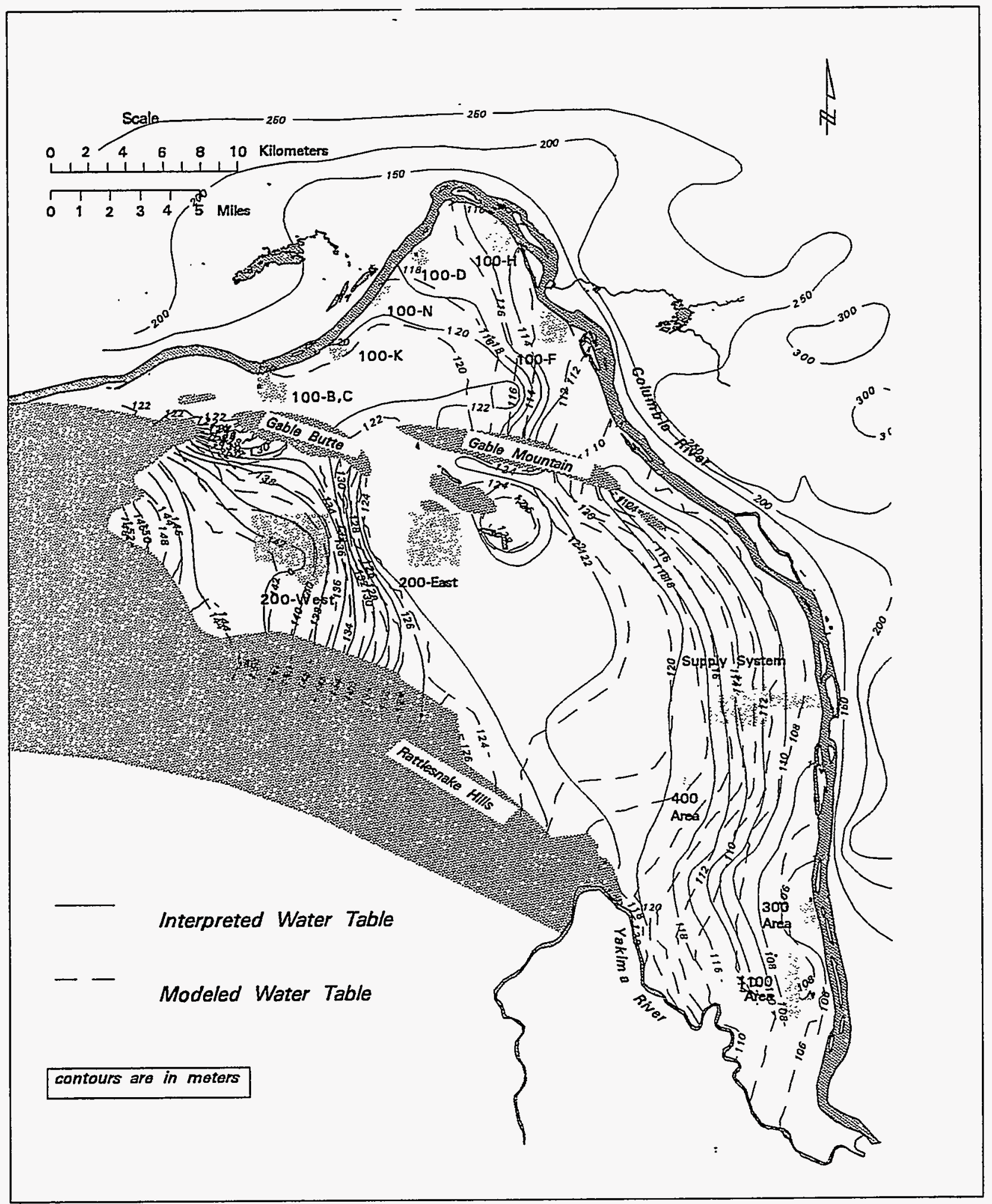

Figure 4. Comparison of Model Results to Interpreted Water Table Contours for June 1992. 



\section{Assumptions and Limitations}

There is a degree of uncertainty inherent in all models that comes from the assumptions made when developing and applying them. Predictions with models can be made confidently for a time period comparable to the period that was matched historically, provided that future conditions do not invalidate some of the model assumptions. In making longer predictions, the cumulative errors arising from making inappropriate assumptions for the conceptual model, model structure, and parameter estimates may become significantly large (Bredehoeft and Konikow 1993).

Many assumptions that are associated with the current Hanford Site regional ground-water model may no longer be appropriate. Since the conceptual model was developed, new hydrogeologic data have been collected, new interpretations have been made, and the hydrogeologic conditions and driving forces (e.g., boundary conditions) at the Hanford Site have changed. The current model was developed based on the assumption that artificial recharge from Site operations was much greater than any natural recharge from precipitation or the basalt aquifers below the unconfined aquifer system at the Hanford Site. In the past, it was reasonable to ignore natural recharge because the flow system was dominated by effects from artificial recharge. However, artificial recharge is presently decreasing and is projected to decrease even more in the future. As the flow system approaches pre-Hanford conditions, this assumption becomes invalid, and it is crucial to include natural recharge in the model to accurately represent the water balance and the driving forces that control water movement.

Additional sources for uncertainty include the use of a constant value for specific yield and a constant "average" head value assigned to the Columbia and Yakima rivers. In addition, the hydraulic conductivity distribution used in the model is based on an averaging of properties across Hanford and Ringold formation layers into a transmissivity value for each element. The flow system response to water being released from storage in the Hanford formation, for example, will be quite different from the response observed in the Ringold sediments because of the difference in hydraulic properties. This assumption may greatly influence the results of a transient twodimensional simulation. In the 100 and 300 Areas, the finite elements are large, resulting in poor resolution for the solution of ground-water flow and the interpolation of results from the CFEST nodes to the well locations. 
The straight-line projection model used in a companion study has limitations because it assumes that water levels will continue to decline at the same rate in the future as they have in the past. As a result, projecting recent changes onto future conditions will not necessarily reflect the water table response to future changes in discharge unless these changes in discharge are like those of the recent past.

Both analysis methods are subject to error in the well screen elevation values. Well screen information is reported in the Hanford Environmental Information System (HEIS) database as depth from ground. However, ground surface elevation is not available for most wells, although casing elevation is. Therefore, well screen elevations were calculated from casing elevations with $2 \mathrm{ft}$ subtracted to provide elevation from ground surface (most Hanford wells have a stick-up height between 2 and $3 \mathrm{ft}$ ). This error is consistent between the two methods since the same well data were used in both. 


\section{Results and Discussion}

Simulation of future flow conditions in the unconfined aquifer system estimate how water level elevations may change in response to projected decreases in effluent discharges. Figures 5 , 6 , and 7 show the predicted water table for 1995, 2000, and 2005. The change (decline) in the water table that is projected to occur from 1993 to 2005 can be seen in Figure 8. The analysis comparing well screen elevations to the water table is presented for 1995, 2000, and 2005. Because of the uncertainty in the model associated with the assumptions previously described, the model should not be relied on for exact dates and water levels. However, it is reasonable to use the modeling results as a semi-quantitative representation of future conditions.

The model results may be assessed by comparison with straight-line method results for the 200 Areas. Figure 9 shows wells located in the 200 East Area that may be affected by declining water levels by the year 2000 as predicted by the CFEST simulations. Results of the straight-line projections for the same time are shown in Figure 10. Most wells fall in the same category for both analysis methods, although in this area the straight-line method tends to predict more wells going dry than does the ground-water model. Figures 11 and 12 show results for the 200 West Area. CFEST simulation results (Figure 11) generally provide higher water levels and therefore predict less wells going dry than does the straight-line projection method (Figure 12).

Appendix B contains maps of selected areas showing wells that may be affected by the change in water table elevation predicted by the model. In general, the predictions indicate that wells in the 100-N, 200-West and B-pond areas will be impacted the most by water level changes. Estimates of the water column in selected wells are presented in tabular form in Appendix C. A few wells were selected to compare with the hydrographs generated from observed data and the straight-line projection analysis (Appendix D). In most cases, the results of the model predictions show wells going dry later than do the results of the straight-line projection. This is because the straight-line projection is an extension of the current rate of decline of the water table. However, in the real system, the water levels in the unconfined aquifer system should approach a steady state asymptotically. Therefore, the straight-line projection method will overestimate the true water level decline until the curve nears steady state. The rate of decrease in discharges at the Site has been greater in the past than it will be in the future, and the straight-line projection method does not account for this. The method using a ground-water model considers this non-linear approach to steady state, and thus, in general, provides a better prediction of future water levels than the 


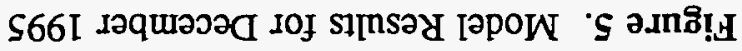

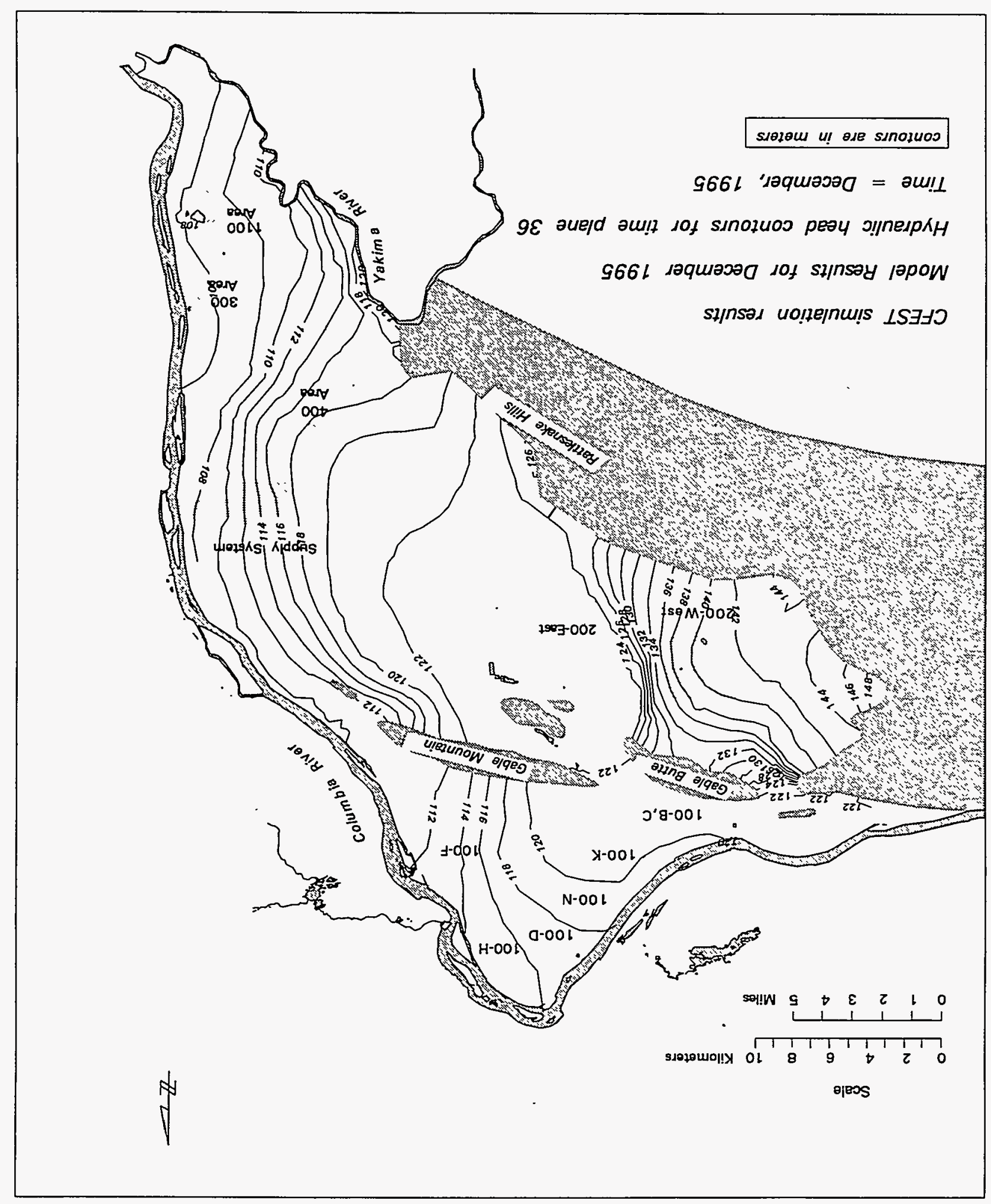




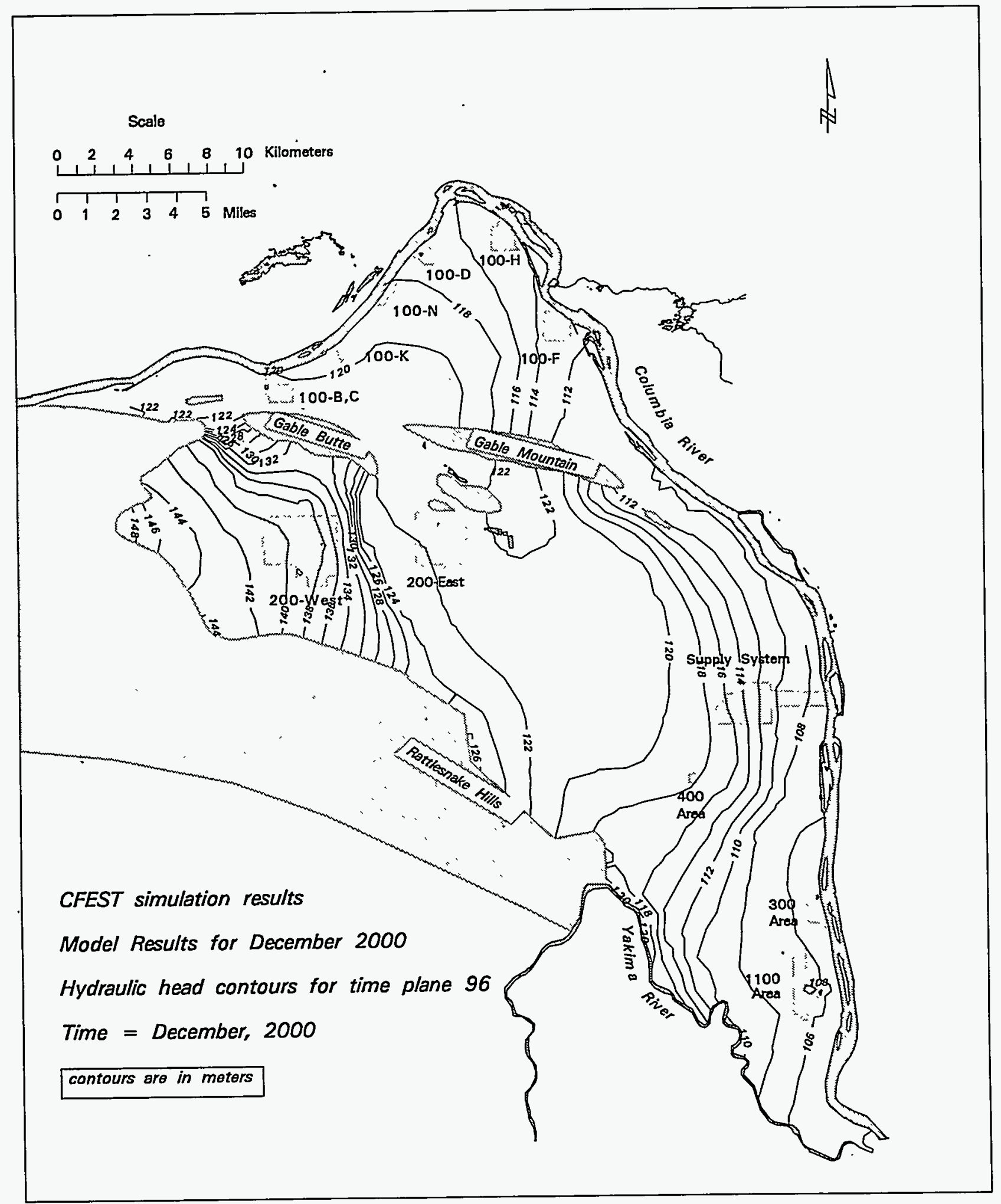

Figure 6. Model Results for December 2000 


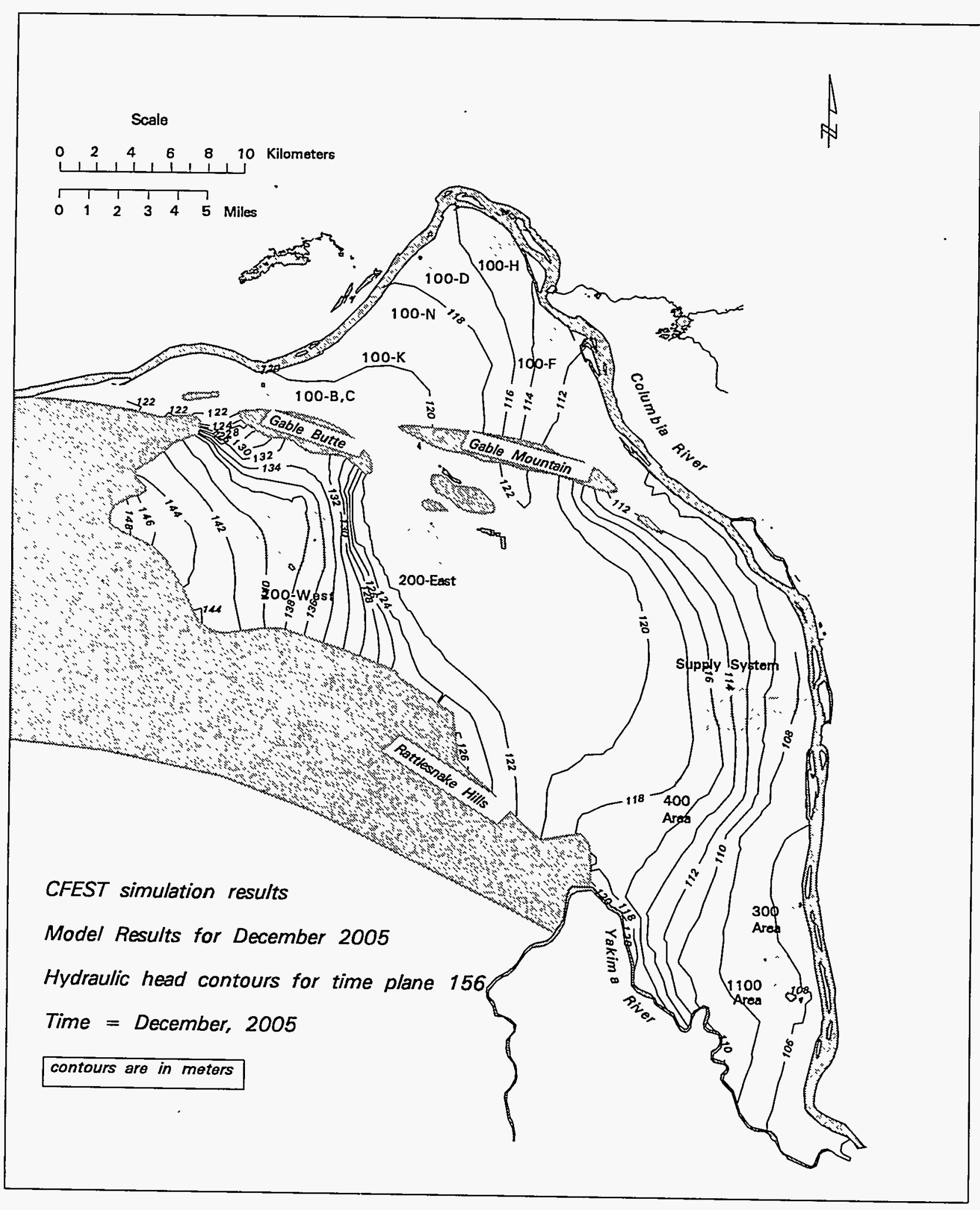

Figure 7. Model Results for December 2005 


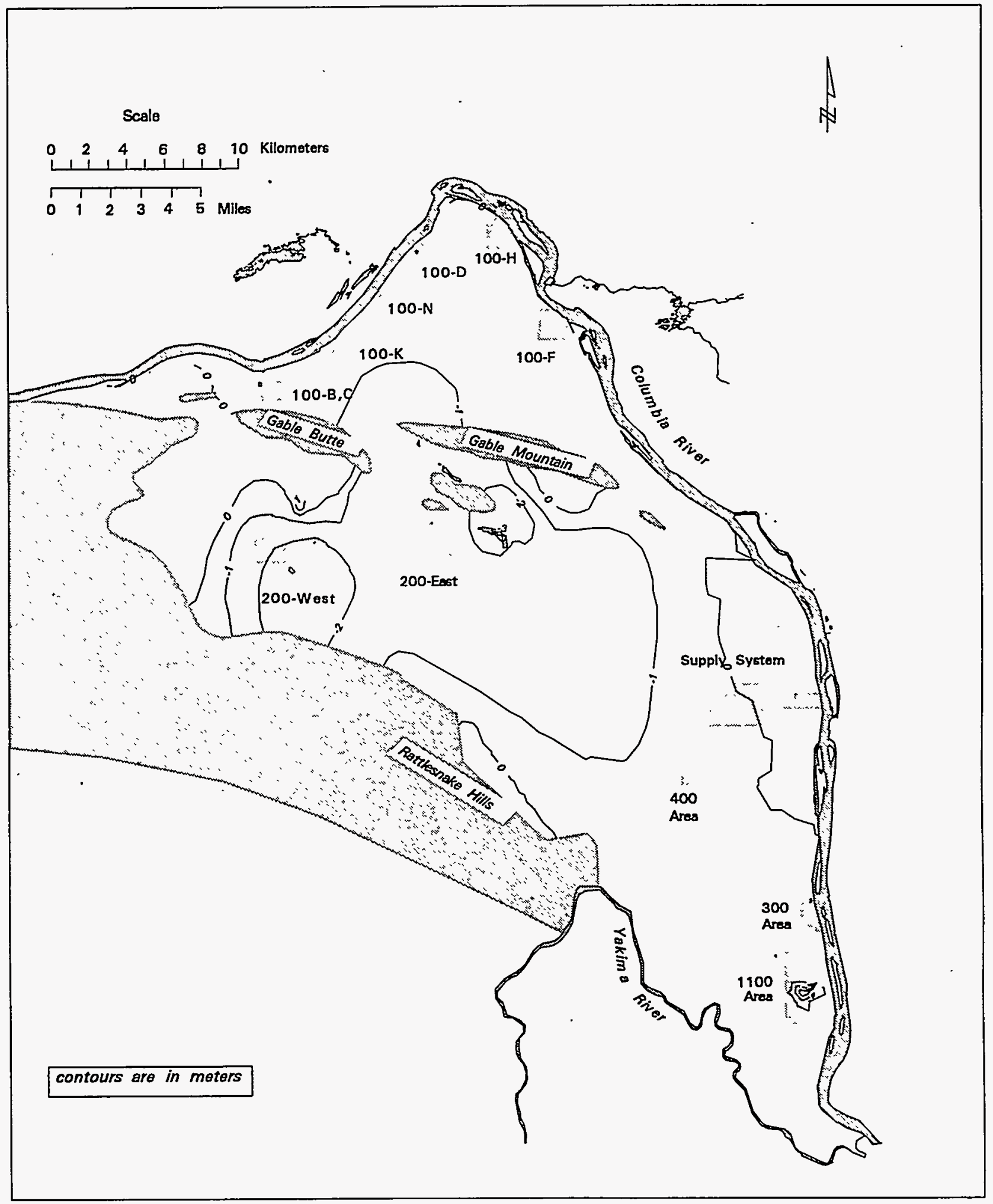

Figure 8. Modeled Water Table Changes From December 1993 to December 2005 


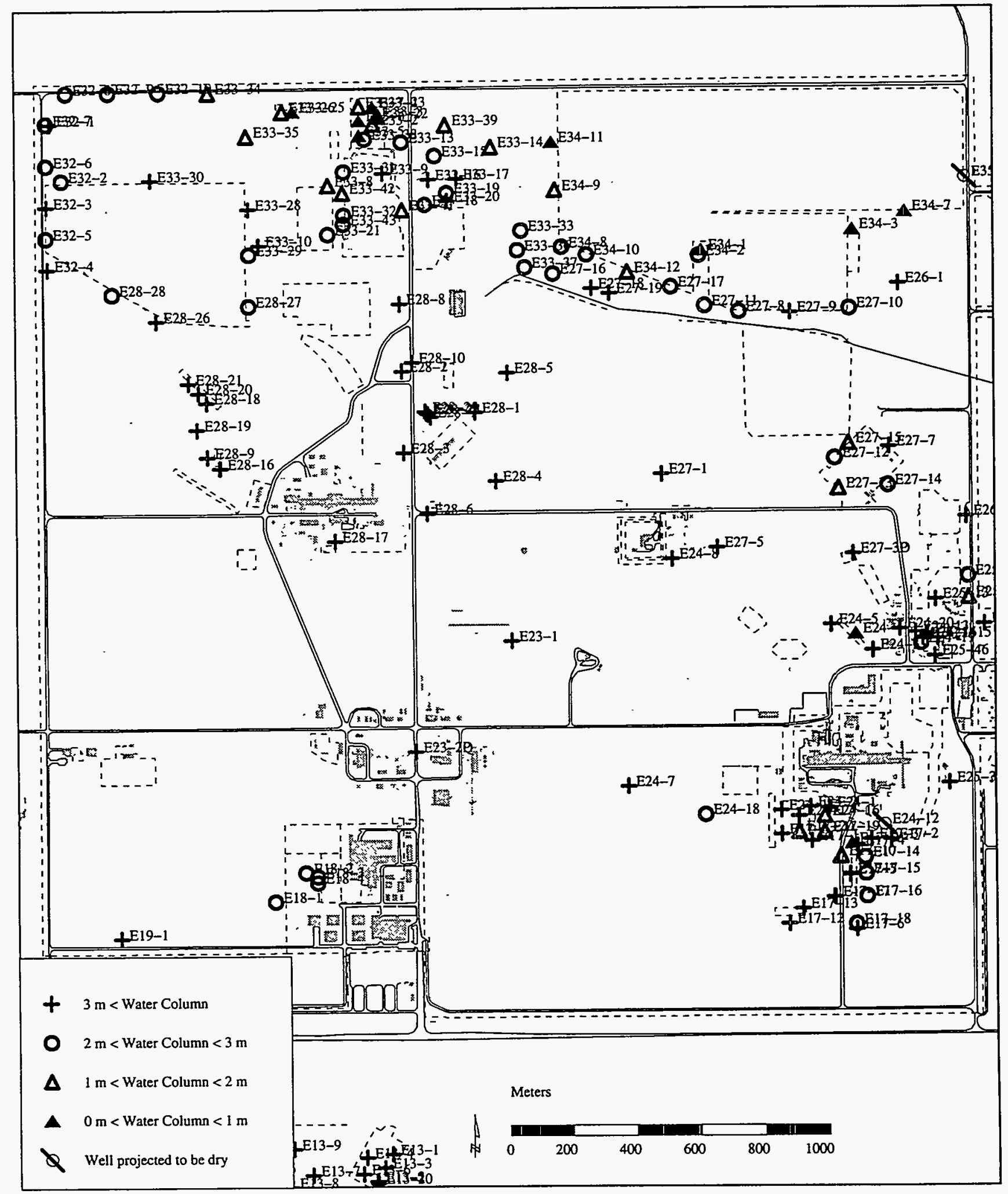

Figure 9. Predicted Impact of Declining Water Levels on 200 East Area Wells for January 2000, Determined Using the Ground-Water Model 


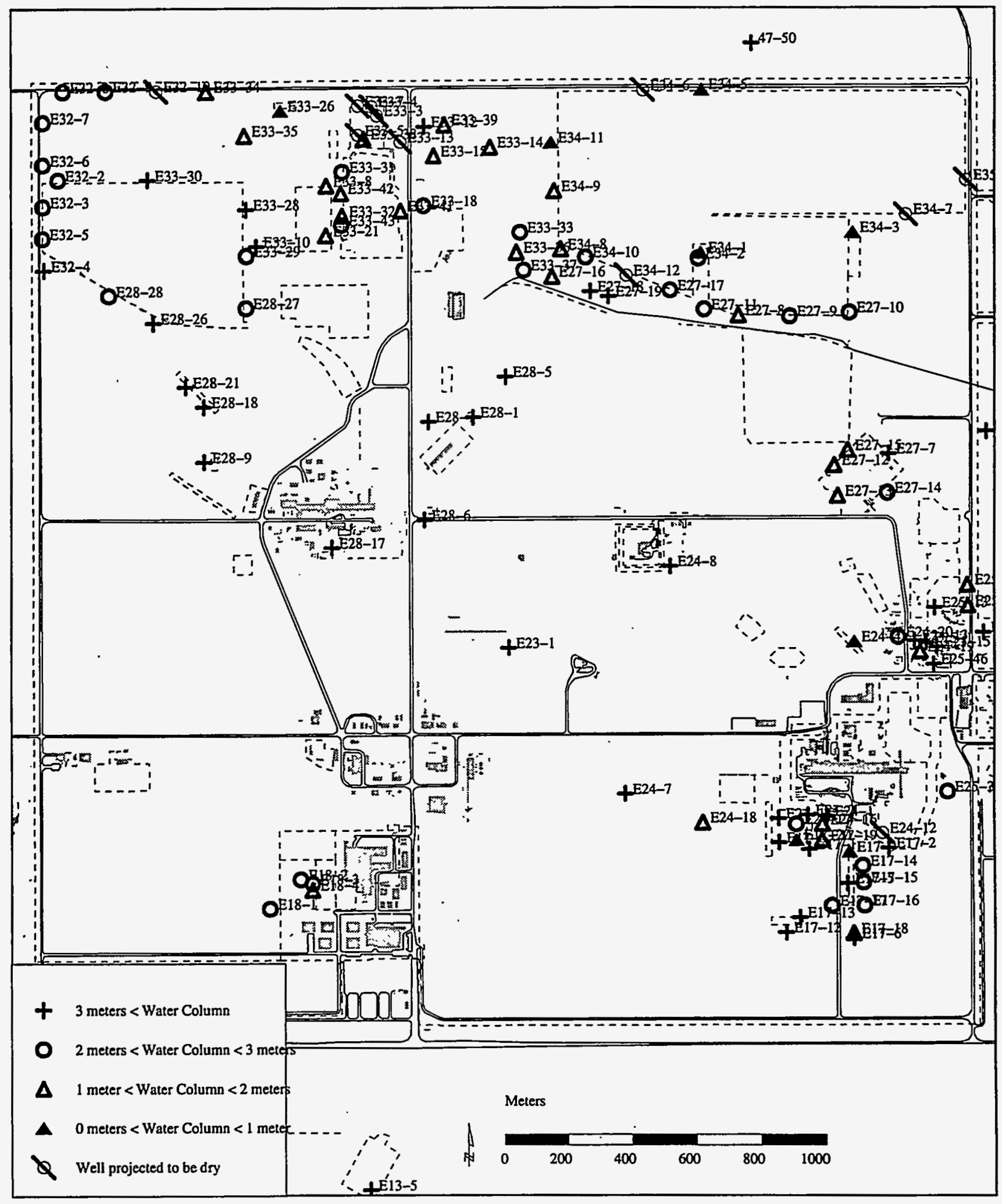

Figure 10. Projected Impact of Declining Water Levels on 200 East Area Wells for January 2000, Determined Using the Straight-Line Projection Method 


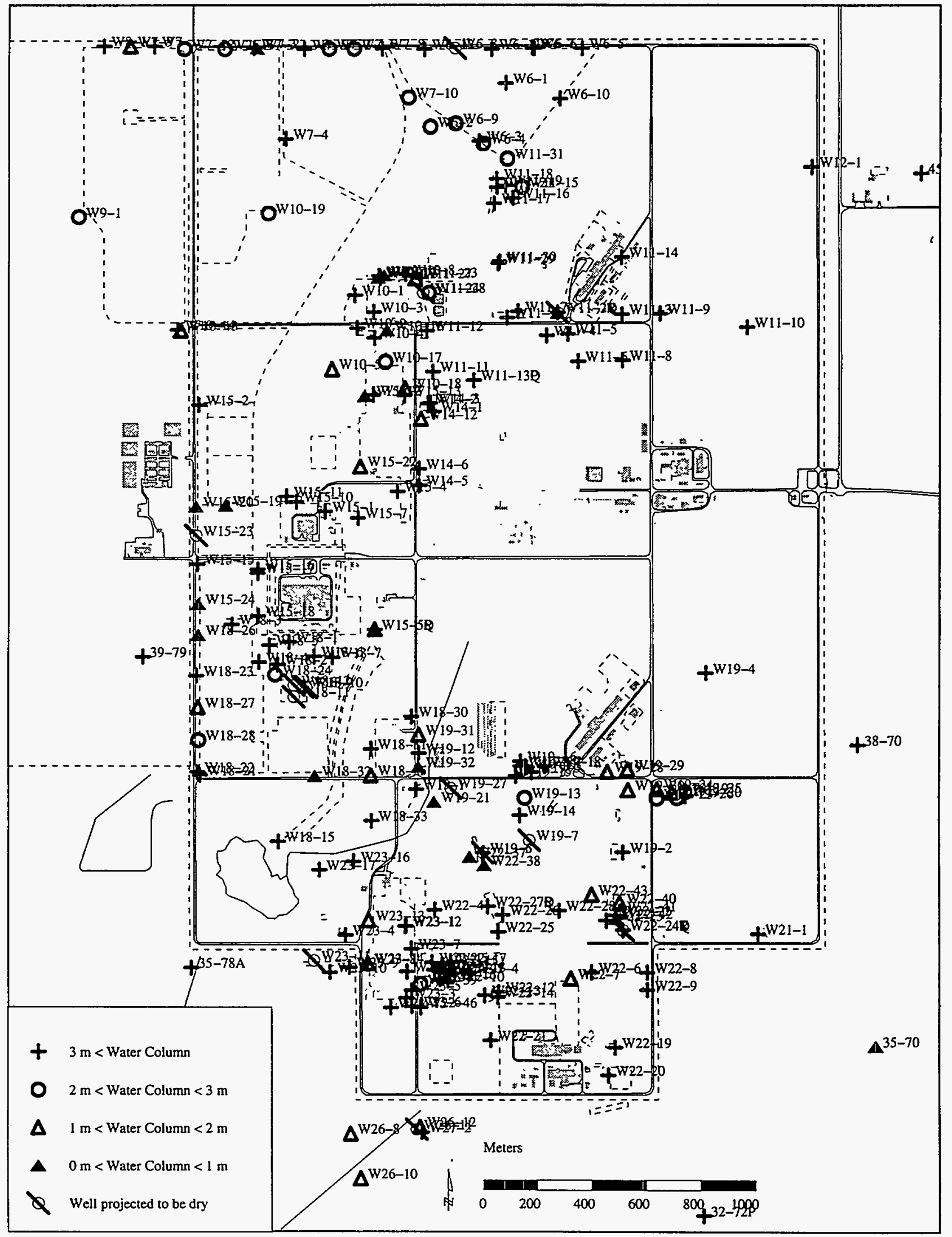

Figure 11. Predicted Impact of Declining Water Levels on 200 West Area Wells for January 2000, Determined Using the Ground-Water Model 


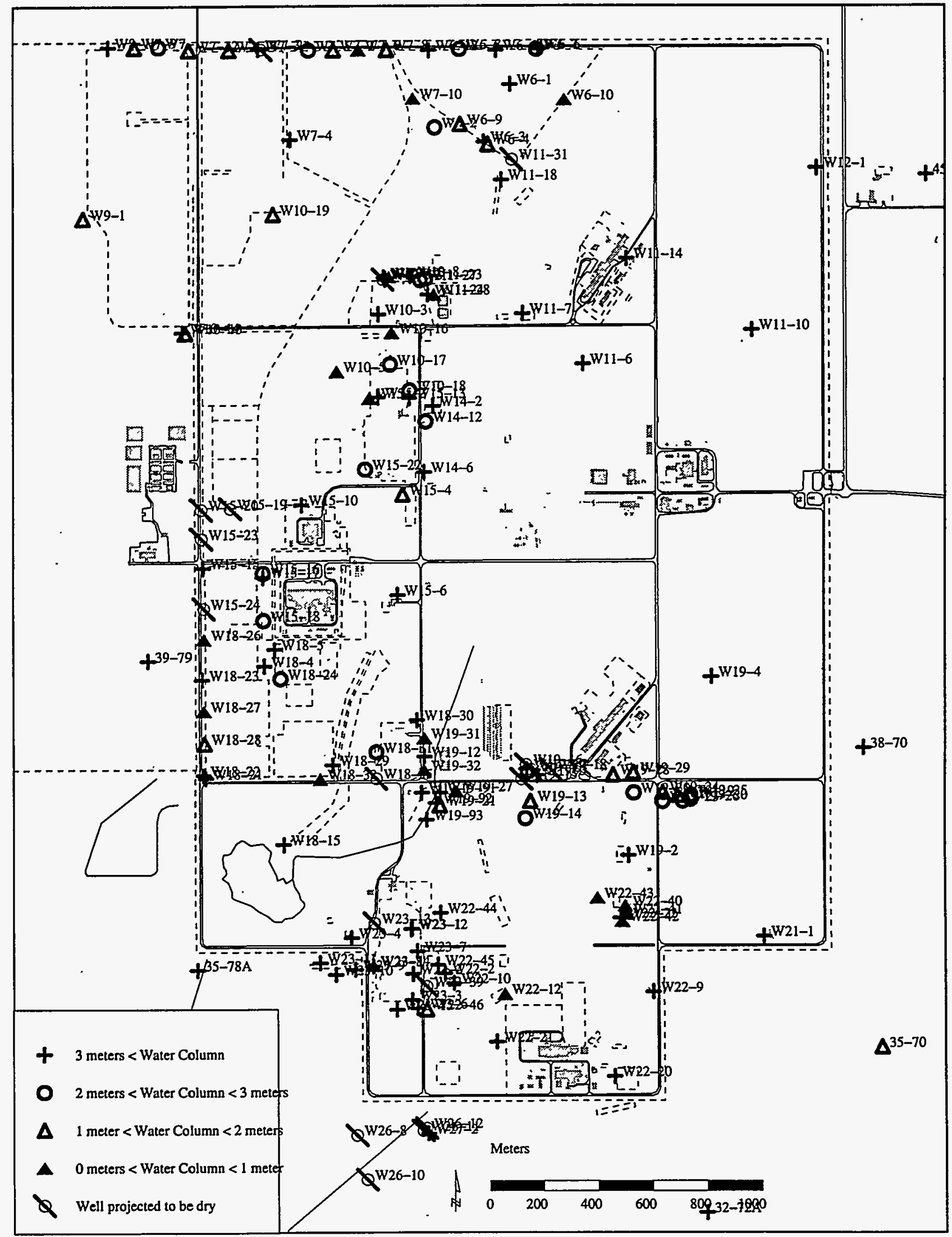

Figure 12. Projected Impact of Declining Water Levels on 200 West Area Wells for January 2000, Determined Using the Straight-Line Projection Model 
straight-line projection method. For one example (well 299-E34-11), the straight-line projection suggests that the water level in the well will be above the bottom of the screen long after the model results show the well going dry. This may be accounted for by the fact that the ground-water model is regional in scale, with the result that some local flow system characteristics may not be captured as well by the model. Both analysis methods are models, so a comparison does not represent a calibration of the ground-water flow model.

It is clear that within the next 5 to 10 years, more wells currently used for monitoring will begin to go dry or will be difficult to sample. Predictions of wells that will go dry within the next 5 years have less uncertainty than predictions of wells that will become dry within 5 to 10 years. Each of these predictions is an estimate based on assumed future Site operational conditions and model assumptions. Future conditions that differ from those used in the model will result in a different hydrologic response.

Dewatering of the unconfined aquifer system in response to decreased wastewater discharge will be transient. Different portions of the aquifer will release water at different times in the future. As Site operations cease, the flow system in the unconfined aquifer will approach conditions similar to pre-Hanford conditions, although irrigation in the Cold Creek Valley area will provide more recharge to the unconfined aquifer than was present during pre-Hanford time.

Zimmerman et al. (1986) observed that storage changes occurred in the unconfined aquifer through 1970 in response to discharges to the ground at the Hanford Site. Similarly, it can be expected that the effects of declining discharges at the Site will be observed in the unconfined aquifer for several decades into the future. 


\section{References}

Bierschenk, W.H. 1957. Hydraulic Characteristics of Hanford Aquifers. HW-48916, General Electric Company, Hanford Atomic Products Operation, Richland Washington.

Bredehoeft, J. D., and L. F. Konikow. 1993. "Ground-Water Models: Validate or invalidate." Editorial in Ground Water, 31(2):178

Chamness, M. A., and J. K. Merz. 1993. Hanford Wells. PNL-8800, Pacific Northwest Laboratory, Richland, Washington.

Freeze, R. A., and J. A. Cherry. 1979. Groundwater. Prentice-Hall, Inc., Englewood Cliffs, New Jersey.

Gee, G. W., M. J. Fayer, M. L. Rockhold, and M. D. Campbell. 1992. "Variations in Recharge at the Hanford Site." Northwest Science, 66(4): 237.

Graham, M.J., M.D. Hall, S.R. Strait, and W.R. Brown. 1981. Hydrology of the Separations Area. RHO-ST-42, Rockwell Hanford Operations, Richland, Washington.

Jacobson, E. A., and M. D. Freshley. 1990. An Initial Inverse Calibration of the Ground-Water Flow Model for the Hanford Unconfined Aquifer. PNL-7144, Pacific Northwest Laboratory, Richland, Washington.

Kipp, K.L. and R.D. Mudd. 1973. Collection and Analysis of Pump Test Data for Transmissivity Values. BNWL-1709, Pacific Northwest Laboratory, Richland, Washington.

Newcomer, D. R. 1990. Evaluation of Hanford Site Water-Table Changes - 1980 to 1990. PNL7498, Pacific Northwest Laboratory, Richland, Washington.

Swanson, L. C. 1992. Phase 1 Hydrogeologic Summary of the 300-FF-5 Operable Unit, 300 Area. WHC-SD-EN-TI-052, Westinghouse Hanford Company, Richland, Washington.

Swanson, L. C., S. P. Reidel, K. A. Lindsey, and D. J. Anderson. 1994. 1994 Characterization Report for the Proposed State-Approved Land Disposal Site. WHC-SD-C018H-RPT-003, Westinghouse Hanford Company, Richland, Washington.

Thorne, P. D., and D. R. Newcomer. 1992. Summary and Evaluation of Available Hydraulic Property.Data for the Hanford Site Unconfined Aquifer System. PNL-8337, Pacific Northwest Laboratory, Richland, Washington..

Woodruff, R. K., R. W. Hanf, and R. E. Lundgren. 1993. Hanford Site Environmental Report for Calendar Year 1992. PNL-8682, Pacific Northwest Laboratory, Richland, Washington.

Wurstner, S. K., and J. L. Devary. 1993. Hanford Site Ground-Water Model: Geographic Information System Linkages and Model Enhancements, FY 1993. PNL-8991, Pacific Northwest Laboratory, Richland, Washington.

Zimmerman, D. A., A. E. Reisenauer, G. D. Black, and M. A. Young. 1986. Hanford Site Water Table Changes 1950 Through 1980 - Data Observations and Evaluation. PNL-5506, Pacific Northwest Laboratory, Richland; Washington. 
Appendix A

\section{Estimates of Effluent Discharge Volumes for the Hanford Site}




\begin{tabular}{|c|c|c|c|c|c|c|}
\hline \multirow[b]{2}{*}{$\begin{array}{l}\text { Release } \\
\text { Site }\end{array}$} & \multicolumn{2}{|c|}{1980} & \multicolumn{2}{|c|}{$1981^{\circ}$} & \multicolumn{2}{|c|}{1982} \\
\hline & $\begin{array}{c}\text { Average } \\
\text { Effluent } \\
\text { Volume }(\mathrm{m} 3 / \mathrm{d})\end{array}$ & $\begin{array}{l}\text { Data Quality } \\
\text { Indicator }\end{array}$ & $\begin{array}{c}\text { Average } \\
\text { Effluent } \\
\text { Volume (m3/a) }\end{array}$ & $\begin{array}{l}\text { Data Quality } \\
\text { Indicator }\end{array}$ & $\begin{array}{c}\text { Average } \\
\text { Effluent } \\
\text { Volume (m3/d) }\end{array}$ & $\begin{array}{l}\text { Data Quality } \\
\text { Indicator }\end{array}$ \\
\hline $216-A-10$ & $0.00 E+00$ & Good & $1.63 \mathrm{E}-01$ & Good & $3.44 E+01$ & Good \\
\hline $216-A-25$ & $2.72 E+04$ & Good Estimate & $1.69 \mathrm{E}+04$ & Good Estimate & $1.89 E+04$ & Good Estimate \\
\hline $216-A-3$ & $8.04 \mathrm{E}-02$ & Estimate (?) & $4.30 \mathrm{E}-02$ & Good & $0.00 \mathrm{E}+00$ & Good \\
\hline $216-\mathrm{A}-30$ & $2.30 \mathrm{E}+02$ & Good & $4.18 \mathrm{E}+02$ & Good & $4.77 E+02$ & Good \\
\hline $216-\mathrm{A}-36 \mathrm{~B}$ & $0.00 E+00$ & Good & $0.00 \mathrm{E}+00$ & Good & $6.50 E+00$ & Good \\
\hline $216-\mathrm{A}-37-1$ & $8.23 E+01$ & Good & $2.67 E+01$ & Good & $4.00 \mathrm{E}+01$ & Good \\
\hline $216-A-37-2$ & $0.00 \mathrm{E}+00$ & Good & $0.00 \mathrm{E}+00$ & Good & $0.00 \mathrm{E}+00$ & Good \\
\hline $216-A-45$ & $0.00 \mathrm{E}+00$ & Good & $0.00 \mathrm{E}+00$ & Good & $0.00 \mathrm{E}+00$ & Good \\
\hline $216-A-8$ & $0.00 \mathrm{E}+00$ & Good & $0.00 E+00$ & Good & $0.00 \mathrm{E}+00$ & Good \\
\hline $216-B-3$ & $1.20 E+04$ & Good & $1.67 \mathrm{E}+04$ & Good & $1.42 \mathrm{E}+04$ & Good \\
\hline $216-\mathrm{B}-55$ & $1.11 E+02$ & Good & $1.12 E+02$ & Good & $5.65 \mathrm{E}+01$ & Good \\
\hline $216-B-62$ & $4.32 E+01$ & Good & $4.89 E+01$ & Good & $3.36 \mathrm{E}+01$ & Good \\
\hline $216-B-63$ & $8.90 \mathrm{E}+02$ & Good & $9.77 \mathrm{E}+02$ & Good & $8.60 \mathrm{E}+02$ & Good \\
\hline $216-5-10$ & $5.04 \mathrm{E}+02$ & Estimate(?) & $5.45 \mathrm{E}+02$ & Estimate (2) & $5.44 \mathrm{E}+02$ & Estimate (?) \\
\hline $216-5-19$ & $1.41 E+02$ & Good & $2.35 E+02$ & Good & $1.25 E+02$ & Good \\
\hline $216-S-25$ & $6.94 \mathrm{E}+01$ & Good & $0.00 \mathrm{E}+00$ & Good & $0.00 \mathrm{E}+00$ & Good \\
\hline $216-s-26$ & $0.00 \mathrm{E}+00$ & Good & $0.00 \mathrm{E}+00$ & Good & $0.00 \mathrm{E}+00$ & Good \\
\hline $216-U-10$ & $9.76 \mathrm{E}+03$ & Good Estimate & $5.19 \mathrm{E}+03$ & Good Estimate & $1.36 \mathrm{E}+03$ & Good Estimate \\
\hline $216-U-12$ & $0.00 \mathrm{E}+00$ & Good & $4.38 \mathrm{E}-02$ & Good & $5.18 \mathrm{E}-01$ & Good \\
\hline $216-U-14$ & $0.00 \mathrm{E}+00$ & Estimate & $0.00 \mathrm{E}+00$ & Estimate & $0.00 E+00$ & Estimate \\
\hline $216-U-16$ & $0.00 \mathrm{E}+00$ & Good & $0.00 \mathrm{E}+00$ & Good & $0.00 \mathrm{E}+00$ & Good \\
\hline $216-U-17$ & $0.00 \mathrm{E}+00$ & Good & $0.00 \mathrm{E}+00$ & Good & $0.00 \mathrm{E}+00$ & Good \\
\hline $216-W-L C$ & $0.00 \mathrm{E}+00$ & Good & $1.23 \mathrm{E}+02$ & Good & $4.90 E+02$ & Good \\
\hline $216-z-20$ & $0.00 \mathrm{E}+00$ & Good & $3.00 \mathrm{E}+02$ & Good & $1.04 \mathrm{E}+03$ & Good \\
\hline $\begin{array}{l}\text { North Richland } \\
\text { Well Field } \\
\text { Wellom }\end{array}$ & $1.69 E+04$ & Average & $1.72 E+04$ & Estimate & ש & Est1mate \\
\hline
\end{tabular}

Data Qual1ty Indlcators:

Good = All indications are that the data value is the actual amount released

Good Estimate $=$ a reliable estimate

Estimate = an estimate, but not as reliable as a "good estimate"

Estimate(?) = There is reason to suspect that the data value may be an estimate

Average $=$ Data value is some type of average

Poor $=$ Data is most likely inaccurate 


\begin{tabular}{|c|c|c|c|c|c|c|}
\hline \multirow[b]{2}{*}{$\begin{array}{l}\text { Release } \\
\text { site }\end{array}$} & \multicolumn{2}{|c|}{1983} & \multicolumn{2}{|c|}{1984} & \multicolumn{2}{|c|}{1985} \\
\hline & $\begin{array}{c}\text { Average } \\
\text { Effluent } \\
\text { Volume (m } 3 / d)\end{array}$ & $\begin{array}{l}\text { Data Quality } \\
\text { Indicator }\end{array}$ & $\begin{array}{c}\text { Average } \\
\text { Effluent } \\
\text { Volume }(\mathrm{m} 3 / \mathrm{d})\end{array}$ & $\begin{array}{l}\text { Data Quality } \\
\text { Indicator }\end{array}$ & $\begin{array}{c}\text { Average } \\
\text { Effluent } \\
\text { volume (m3/d) }\end{array}$ & $\begin{array}{l}\text { Data Quality } \\
\text { Indicator }\end{array}$ \\
\hline $216-A-10$ & $8.41 \mathrm{E}+01$ & Good & $2.83 E+02$ & Good & $2.77 \mathrm{E}+02$ & Good \\
\hline $216-A-25$ & $4.05 E+04$ & Good Estimate & $6.41 E+04$ & Good Estimate & $5.19 E+04$ & Good Estimate \\
\hline $216-\mathrm{A}-3$ & $0.00 \mathrm{E}+00$ & Good & $0.00 E+00$ & Good & $0.00 \mathrm{E}+00$ & Good \\
\hline $216-A-30$ & $4.45 E+02$ & Good Estimate & $7.91 \mathrm{E}+02$ & Good Estimate & $1.30 \mathrm{E}+03$ & Good Estimate \\
\hline $216-A-36 B$ & $2.71 E+01$ & Good & $1.30 \mathrm{E}+02$ & Good & $2.06 \mathrm{E}+02$ & Good \\
\hline $216-A-37-1$ & $3.93 \mathrm{E}+01$ & Good & $1.46 \mathrm{E}+02$ & Good & $1.34 E+02$ & Good \\
\hline $216-A-37-2$ & $1.67 E+02$ & Good Estimate & $3.96 \mathrm{E}+02$ & Good Estimate & $6.49 \mathrm{E}+02$ & Good Estimate \\
\hline $216-A-45$ & $0.00 \mathrm{E}+00$ & Good & $0.00 \mathrm{E}+00$ & Good & $0.00 \mathrm{E}+00$ & Good \\
\hline $216-A-8$ & $3.58 \mathrm{E}-01$ & Good & $2.91 E+00$ & Good & $6.88 \mathrm{E}-01$ & Good \\
\hline $216-B-3$ & $1.93 E+04$ & Good & $1.45 \mathrm{E}+04$ & Good & $1.46 \mathrm{E}+04$ & Estimate \\
\hline $216-B-55$ & $1.05 \mathrm{E}+02$ & Good & $9.28 \mathrm{E}+01$ & Good & $2.44 \mathrm{E}+01$ & Good \\
\hline $216-\mathrm{B}-62$ & $6.52 \mathrm{E}+01$ & Good & $1.91 E+01$ & Good & $1.07 \mathrm{E}+01$ & Good \\
\hline $216-B-63$ & $8.51 \mathrm{E}+02$ & Good & $8.26 \mathrm{E}+02$ & Good & $8.52 \mathrm{E}+02$ & Good \\
\hline $216-s-10$ & $5.46 \mathrm{E}+02$ & Estimate (?) & $5.46 \mathrm{E}+02$ & Est1mate (?) & $5.55 \mathrm{E}+02$ & Estimate (?) \\
\hline $216-s-19$ & $1.77 \mathrm{E}+02$ & Good & $9.74 \mathrm{E}+01$ & Good & $0.00 \mathrm{E}+00$ & Good \\
\hline $216-s-25$ & $0.00 \mathrm{E}+00$ & Good & $0.00 \mathrm{E}+00$ & Good & $8.64 E+01$ & Good \\
\hline $216-s-26$ & $0.00 \mathrm{E}+00$ & Good & $3.15 E+01$ & Good & $1.23 E+02$ & Good \\
\hline $216-U-10$ & $9.65 \mathrm{E}+02$ & Good Estimate & $3.24 \mathrm{E}+03$ & Good Estimate & $0.00 \mathrm{E}+00$ & Good \\
\hline $216-U-12$ & $3.49 \mathrm{E}+00$ & Good & $1.50 \mathrm{E}+01$ & Good & $1.30 \mathrm{E}+01$ & Good \\
\hline $216-U-14$ & $0.00 \mathrm{E}+00$ & Estimate & $0.00 \mathrm{E}+00$ & Estimate & $1.15 \mathrm{E}+03$ & Good \\
\hline $216-U-16$ & $0.00 \mathrm{E}+00$ & Good & $7.81 \mathrm{E}+02$ & Good & $3.50 \mathrm{E}+02$ & Good \\
\hline $216-U-17$ & $0.00 \mathrm{E}+00$ & Good & $0.00 \mathrm{E}+00$ & Good & $0.00 \mathrm{E}+00$ & Good \\
\hline 216-W-LC & $1.07 \mathrm{E}+03$ & Good & $2.36 \mathrm{E}+02$ & Good & $1.98 \mathrm{E}+02$ & Good \\
\hline $216-z-20$ & $1.44 \mathrm{E}+03$ & Good & $1.90 E+03$ & Good & $1.06 \mathrm{E}+03$ & Good \\
\hline $\begin{array}{l}\text { North Richland } \\
\text { Well Field }\end{array}$ & $1.31 E+04$ & Estimate & $2.29 E+04$ & Good Estimate & $2.02 E+04$ & Good Est1mate \\
\hline
\end{tabular}

Data Quality Indicators:

Good $=$ All indications are that the data value is the actual amount released

Good Estimate = a reliable estimate

Estimate = an estimate, but not as reliable as a "good estimate"

Estimate(?) = There is reason to suspect that the data value may be an estimate

Average $=$ Data value is some type of average

Poor = Data is most likely inaccurate 


\begin{tabular}{|c|c|c|c|c|c|c|}
\hline \multirow[b]{2}{*}{$\begin{array}{l}\text { Release } \\
\text { Site }\end{array}$} & \multicolumn{2}{|c|}{1986} & \multicolumn{2}{|c|}{1987} & \multicolumn{2}{|c|}{1988} \\
\hline & $\begin{array}{c}\text { Average } \\
\text { Effluent } \\
\text { Volume (m3/d) }\end{array}$ & $\begin{array}{l}\text { Data Quality } \\
\text { Indicator }\end{array}$ & $\begin{array}{c}\text { Average } \\
\text { Ef fluent } \\
\text { Volume }(\mathrm{m} 3 / d)\end{array}$ & $\begin{array}{l}\text { Data Quality } \\
\text { Indicator }\end{array}$ & $\begin{array}{c}\text { Average } \\
\text { Effluent } \\
\text { volume }(\mathrm{m} 3 / \mathrm{d})\end{array}$ & $\begin{array}{l}\text { Data Quailty } \\
\text { Indicator }\end{array}$ \\
\hline $216-A-10$ & $2.02 E+02$ & Good & $1.74 E+01$ & Good & $0.00 \mathrm{E}+00$ & Good \\
\hline $216-A-25$ & $0.00 \mathrm{E}+00$ & Poor & $0.00 \mathrm{E}+00$ & Good & $0.00 E+00$ & Good \\
\hline $216-A-3$ & $0.00 E+00$ & Good & $0.00 \mathrm{E}+00$ & Good & $0.00 \mathrm{E}+00$ & Good \\
\hline $216-A-30$ & $1.40 E+03$ & Good & $1.09 E+03$ & Good Estimate & $6.81 E+02$ & Good \\
\hline $216-A-36 B$ & $1.55 E+02$ & Good & $7.64 \mathrm{E}+01$ & Good & $0.00 \mathrm{E}+00$ & Good \\
\hline $216-A-37-1$ & $1.37 \mathrm{E}+02$ & Good & $6.77 E+01$ & Good & $1.34 \mathrm{E}+02$ & Good \\
\hline $216-A-37-2$ & $6.98 \mathrm{E}+02$ & Good & $5.37 \mathrm{E}+02$ & Good Estimate & $3.35 E+02$ & Good \\
\hline $216-A-45$ & $0.00 \mathrm{E}+00$ & Good & $1.22 \mathrm{E}+02$ & Good & $1.36 \mathrm{E}+02$ & Good \\
\hline $216-A-8$ & $0.00 \mathrm{E}+00$ & Good & $0.00 \mathrm{E}+00$ & Good & $0.00 \mathrm{E}+00$ & Good \\
\hline $216-B-3$ & $6.24 \mathrm{E}+04$ & Good Estimate & $5.39 \mathrm{E}+04$ & Estimate & $7.30 \mathrm{E}+04$ & Good Estimate \\
\hline $216-B-55$ & $1.17 \mathrm{E}+01$ & Good & $0.00 E+00$ & Good & $7.23 E+00$ & Good \\
\hline $216-\mathrm{B}-62$ & $1.07 E+01$ & Good & $0.00 \mathrm{E}+00$ & Good & $0.00 \mathrm{E}+00$ & Good \\
\hline $216-B-63$ & $1.41 E+03$ & Good & $9.59 \mathrm{E}+02$ & Good & $7.90 \mathrm{E}+02$ & Good \\
\hline $216-S-10$ & $5.45 E+02$ & Est1mate (?) & $5.45 E+02$ & Estimate (?) & $5.75 E+02$ & Estimate (?) \\
\hline $216-s-19$ & $0.00 \mathrm{E}+00$ & Good & $0.00 \mathrm{E}+00$ & Good & $0.00 \mathrm{E}+00$ & Good \\
\hline $216-S-25$ & $0.00 E+00$ & Good & $0.00 \mathrm{E}+00$ & Good & $0.00 \mathrm{E}+00$ & Good \\
\hline $216-s-26$ & $1.13 E+02$ & Good & $9.41 E+01$ & Good & $5.17 E+01$ & Good \\
\hline $216-U-10$ & $0.00 \mathrm{E}+00$ & Good & $0.00 \mathrm{E}+00$ & Good & $0.00 E+00$ & Good \\
\hline $216-U-12$ & $1.08 \mathrm{E}+01$ & Good & $1.76 \mathrm{E}+00$ & Good & $2.85 \mathrm{E}-01$ & Good \\
\hline $216-U-14$ & $1.49 E+03$ & Good & $9.81 \mathrm{E}+02$ & Good & $7.86 \mathrm{E}+02$ & Good \\
\hline $216-U-16$ & $0.00 \mathrm{E}+00$ & Good & $0.00 \mathrm{E}+00$ & Good & $0.00 \mathrm{E}+00$ & Good \\
\hline $216-U-17$ & $0.00 \mathrm{E}+00$ & Good & $0.00 \mathrm{E}+00$ & Good & $2.00 \mathrm{E}+00$ & Good \\
\hline $216-W-L C$ & $1.22 \mathrm{E}+02$ & Good & $1.66 \mathrm{E}+02$ & Good & $3.84 \mathrm{E}+01$ & Good \\
\hline $216-2-20$ & $8.14 \mathrm{E}+02$ & Good & $5.61 \mathrm{E}+02$ & Good & $6.02 \mathrm{E}+02$ & Good \\
\hline $\begin{array}{l}\text { North Richland } \\
\text { Well Field }\end{array}$ & $1.94 \mathrm{E}+04$ & Good Estimate & $1.48 \mathrm{E}+04$ & Good Estimate & $1.00 \mathrm{E}+04$ & Good \\
\hline
\end{tabular}

Data Qual1ty Indicatoxe:

Good = All indications are that the data value is the actual amount released

Good Estimate $=$ a reliable estimate

Estimate = an estimate, but not as reliable as a "good estimate"

Estimate(?) = There is reason to suspect that the data value may be an estimate

Average $=$ Data value is some type of average

Poor $=$ Data is most likely inaccurate 


\begin{tabular}{|c|c|c|c|c|c|c|}
\hline \multirow[b]{2}{*}{$\begin{array}{l}\text { Release } \\
\text { Site }\end{array}$} & \multicolumn{2}{|c|}{1989} & \multicolumn{2}{|c|}{1990} & \multicolumn{2}{|c|}{1991} \\
\hline & $\begin{array}{c}\text { Average } \\
\text { Ef } f \text { luent } \\
\text { Volume }(\mathrm{m} 3 / \mathrm{d})\end{array}$ & $\begin{array}{l}\text { Data Quality } \\
\text { Indicator }\end{array}$ & $\begin{array}{c}\text { Average } \\
\text { Effluent } \\
\text { Volume }(m 3 / d)\end{array}$ & $\begin{array}{l}\text { Data Quality } \\
\text { Indicator }\end{array}$ & $\begin{array}{c}\text { Average } \\
\text { Ef fluent } \\
\text { Volume }(\mathrm{m} 3 / \mathrm{d})\end{array}$ & $\begin{array}{l}\text { Data Quality } \\
\text { Indicator }\end{array}$ \\
\hline $216-A-10$ & $0.00 E+00$ & Good & $0.00 \mathrm{E}+00$ & Good & $0.00 \mathrm{E}+00$ & Good \\
\hline $216-A-25$ & $0.00 \mathrm{E}+00$ & Good & $0.00 \mathrm{E}+00$ & Good & $0.00 \mathrm{E}+00$ & Good \\
\hline $216-A-3$ & $0.00 \mathrm{E}+00$ & Good & $0.00 \mathrm{E}+00$ & Good & $0.00 \mathrm{E}+00$ & Good \\
\hline $216-A-30$ & $9.44 \mathrm{E}+02$ & Good Estimate & $6.15 E+02$ & Good Est1mate & $4.92 \mathrm{E}+02$ & Good Estimate \\
\hline $216-A-36 B$ & $0.00 \mathrm{E}+00$ & Good & $0.00 \mathrm{E}+00$ & Good & $0.00 \mathrm{E}+00$ & Good \\
\hline $216-A-37-1$ & $3.72 \mathrm{E}+01$ & Good & $0.00 \mathrm{E}+00$ & Good & $0.00 \mathrm{E}+00$ & Good \\
\hline $216-A-37-2$ & $4.72 \mathrm{E}+02$ & Good Estimate & $3.08 \mathrm{E}+02$ & Good Estimate & $2.46 E+02$ & Good Estimate \\
\hline $216-A-45$ & $2.48 \mathrm{E}+01$ & Good & $0.00 \mathrm{E}+00$ & Good & $0.00 \mathrm{E}+00$ & Good \\
\hline $216-A-8$ & $0.00 \mathrm{E}+00$ & Good & $0.00 \mathrm{E}+00$ & Good & $0.00 \mathrm{E}+00$ & Good \\
\hline $216-B-3$ & $4.04 E+04$ & Good Estimate & $2.79 E+04$ & Estimate & $2.51 \mathrm{E}+04$ & Estimate \\
\hline $216-8-55$ & $1.79 \mathrm{E}+00$ & Good & $2.65 \mathrm{E}-02$ & Good & $0.00 E+00$ & Good \\
\hline $216-\mathrm{B}-62$ & $0.00 \mathrm{E}+00$ & Good & $0.00 \mathrm{E}+00$ & Good & $0.00 E+00$ & Good \\
\hline $216-B-63$ & $1.05 E+03$ & Good & $6.40 \mathrm{E}+02$ & Good & $7.54 \mathrm{E}+02$ & Good \\
\hline $216-s-10$ & $.5 .99 \mathrm{E}+02$ & Estimate (?) & $5.46 \mathrm{E}+02$ & Estimate (?) & $3.20 \mathrm{E}+02$ & Estimate (?) \\
\hline $216-s-19$ & $0.00 E+00$ & Good & $0.00 \mathrm{E}+00$ & Good & $0.00 \mathrm{E}+00$ & Good \\
\hline $216-s-25$ & $0.00 \mathrm{E}+00$ & Good & $0.00 E+00$ & Good & $0.00 E+00$ & Good \\
\hline $216-s-26$ & $3.62 E+01$ & Good & $3.83 \mathrm{E}+01$ & Good & $2.37 E+01$ & Good \\
\hline $216-\mathrm{U}-10$ & $0.00 \mathrm{E}+00$ & Good & $0.00 \mathrm{E}+00$ & Good & $0.00 \mathrm{E}+00$ & Good \\
\hline $216-U-12$ & $0.00 \mathrm{E}+00$ & Good & $0.00 \mathrm{E}+00$ & Good & $0.00 E+00$ & Good \\
\hline $216-U-14$ & $1.05 \mathrm{E}+03$ & Good & $6.29 \mathrm{E}+02$ & Good & $8.82 \mathrm{E}+02$ & Good \\
\hline $216-U-16$ & $0.00 \mathrm{E}+00$ & Good & $0.00 E+00$ & Good & $0.00 \mathrm{E}+00$ & Good \\
\hline $216-U-17$ & $3.79 \mathrm{E}+00$ & Good & $0.00 \mathrm{E}+00$ & Good & $0.00 \mathrm{E}+00$ & Good \\
\hline 216-W-LC & $6.48 \mathrm{E}+01$ & Good & $8.09 E+01$ & Good & $1.08 \mathrm{E}+02$ & Good \\
\hline $216-z-20$ & $6.88 \mathrm{E}+02$ & Good & $8.28 \mathrm{E}+02$ & Good & $7.44 \mathrm{E}+02$ & Good \\
\hline $\begin{array}{l}\text { North Richland } \\
\text { Well Field }\end{array}$ & $1.82 \mathrm{E}+04$ & Good & 1.30E+04 & Good & $1.41 \mathrm{E}+04$ & Good \\
\hline
\end{tabular}

Data Quality Indicators:

Good = All indications are that the data value is the actual amount released

Good Estimate = a reliable estimate

Estimate = an estimate, but not as reliable as a "good estimate"

Estimate(?) = There is reason to suspect that the data value may be an estimate

Average = Data value is some type of average

Poor $=$ Data is most likely inaccurate 


\begin{tabular}{|c|c|c|}
\hline \multirow[b]{2}{*}{$\begin{array}{l}\text { Release } \\
\text { Site }\end{array}$} & \multicolumn{2}{|c|}{1992} \\
\hline & $\begin{array}{c}\text { Average } \\
\text { Effluent } \\
\text { Volume }(\mathrm{m} 3 / \mathrm{d})\end{array}$ & $\begin{array}{l}\text { Data Quality } \\
\text { Indlcator }\end{array}$ \\
\hline $216-A-10$ & $0.00 \mathrm{E}+00$ & Good \\
\hline $216-A-25$ & $0.00 \mathrm{E}+00$ & Good \\
\hline $216-A-3$ & $0.00 \mathrm{E}+00$ & Good \\
\hline $216-A-30$ & $0.00 \mathrm{E}+00$ & Good \\
\hline $216-A-36 B$ & $0.00 \mathrm{E}+00$ & Good \\
\hline $216-A-37-1$ & $0.00 \mathrm{E}+00$ & Good \\
\hline $216-A-37-2$ & $0.00 \mathrm{E}+00$ & Good \\
\hline $216-A-45$ & $0.00 \mathrm{E}+00$ & Good \\
\hline $216-A-8$ & $0.00 E+00$ & Good \\
\hline $216-B-3$ & $2.14 E+04$ & Estimate \\
\hline $216-B-55$ & $0.00 \mathrm{E}+00$ & Good \\
\hline $216-B-62$ & $0.00 \mathrm{E}+00$ & Good \\
\hline $216-\mathrm{B}-63$ & $1.40 \mathrm{E}+02$ & Good \\
\hline $216-5-10$ & $0.00 \mathrm{E}+00$ & Good \\
\hline $216-5-19$ & $0.00 \mathrm{E}+00$ & Good \\
\hline $216-s-25$ & $0.00 \mathrm{E}+00$ & Good \\
\hline $216-s-26$ & $4.50 \mathrm{E}+01$ & Good \\
\hline $216-U-10$ & $0.00 \mathrm{E}+00$ & Good \\
\hline $216-U-12$ & $0.00 \mathrm{E}+00$ & Good \\
\hline $216-U-14$ & $7.60 \mathrm{E}+02$ & Good \\
\hline $216-\mathrm{U}-16$ & $0.00 \mathrm{E}+00$ & Good \\
\hline $216-\mathrm{U}-17$ & $2.71 \mathrm{E}+00$ & Good \\
\hline $216-W-L C$ & $7.50 \mathrm{E}+01$ & Good \\
\hline $216-z-20$ & $2.45 \mathrm{E}+02$ & Good \\
\hline $\begin{array}{l}\text { North Richland } \\
\text { Well Field }\end{array}$ & $7.07 E+03$ & Good \\
\hline
\end{tabular}

Data Qual1ty Indicators:

Good = All indications are that the data value is the actual amount released

Good Estimate $=$ a reliable estimate

Estimate = an estimate, but not as reliable as a "good estimate"

Estimate(?) = There is reason to suspect that the data value may be an estimate

Average $=$ Data value is some type of average

Poor = Data is most likely inaccurate 


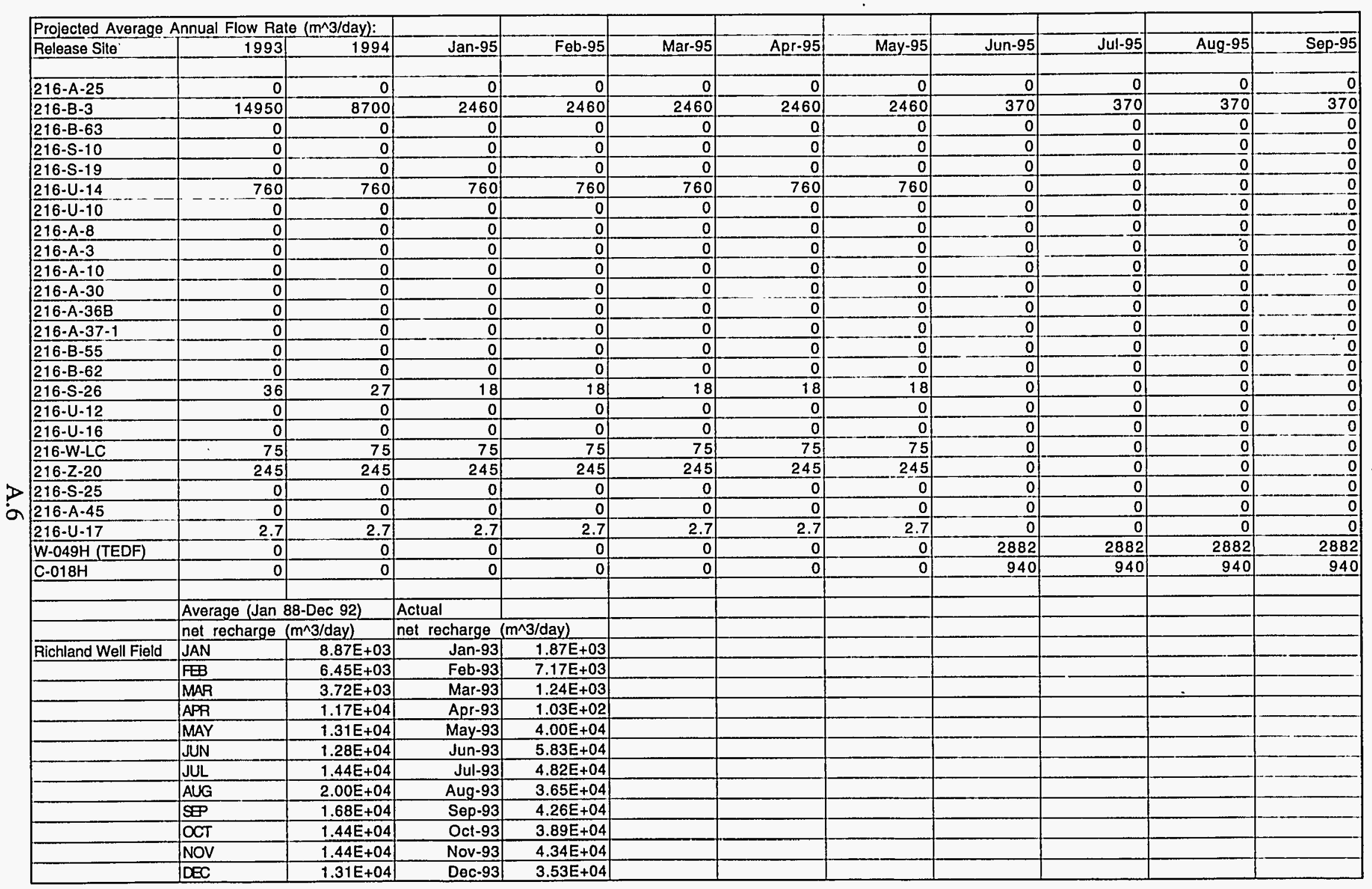




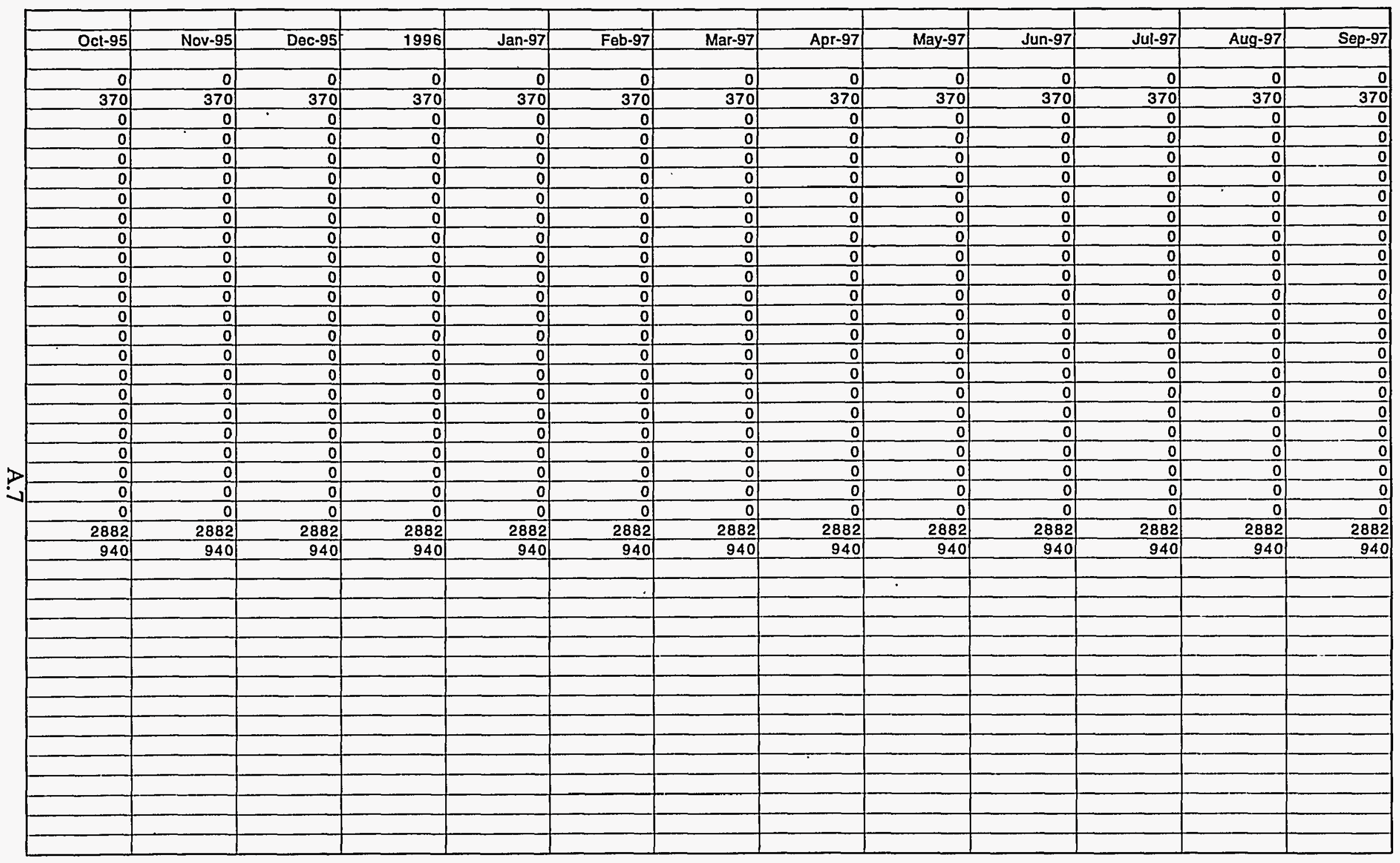




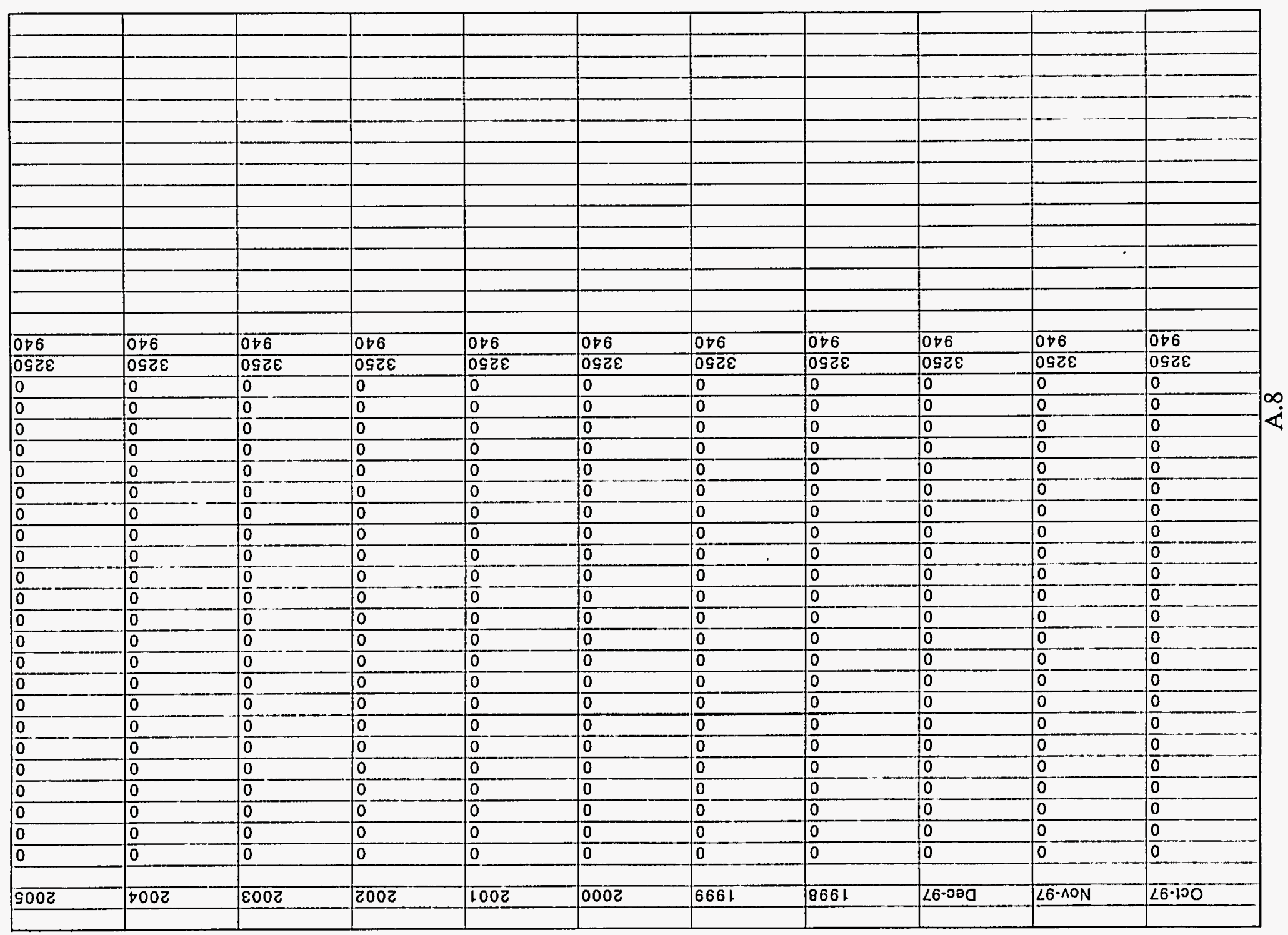

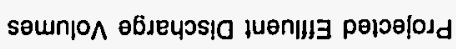




\section{Appendix B}

\section{Maps Showing Wells That May be Affected}

by Declining Water Levels at the Hanford Site 


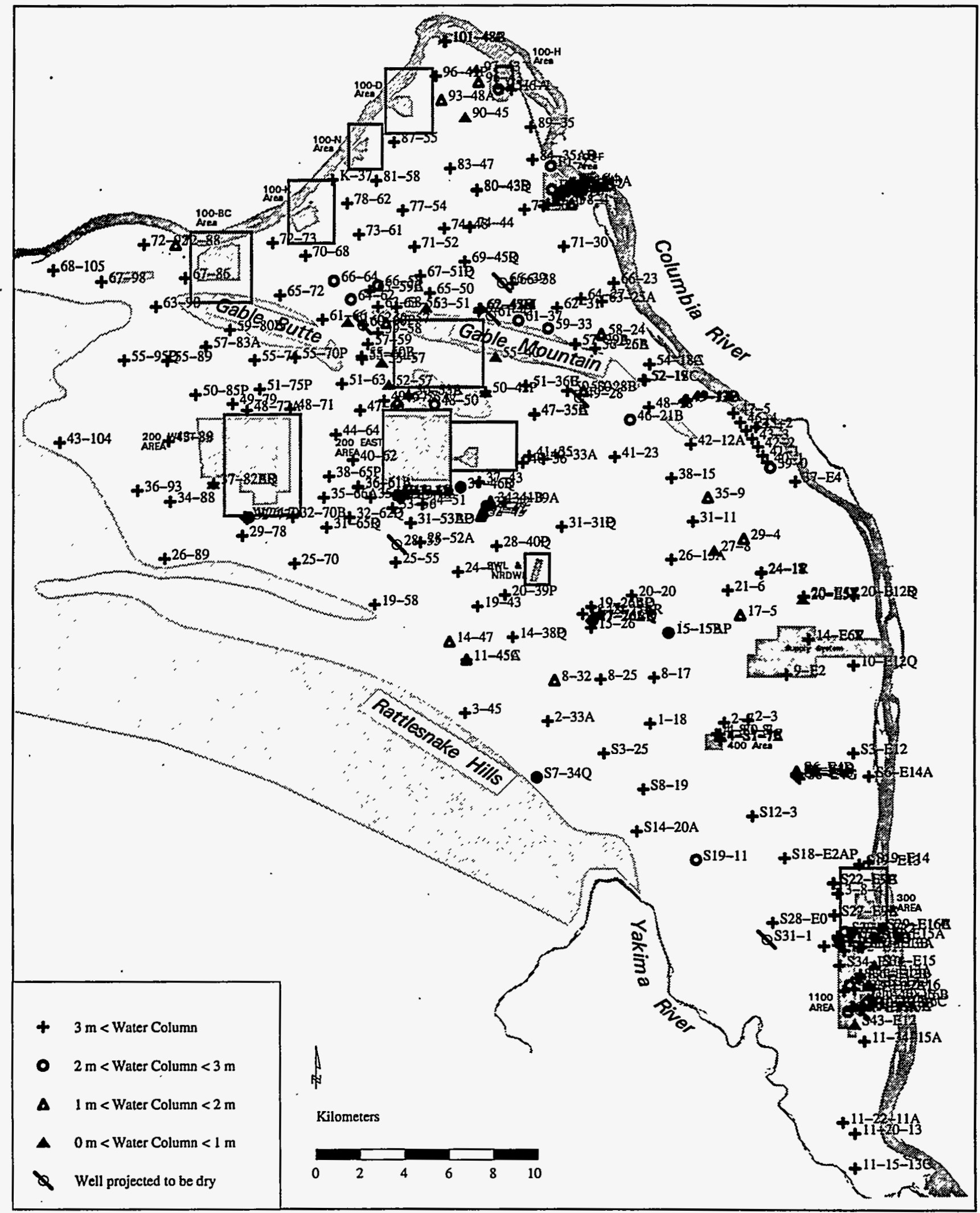

600 Area selected wells which may be impacted by declining water levels before 1 January 1995

B.1 


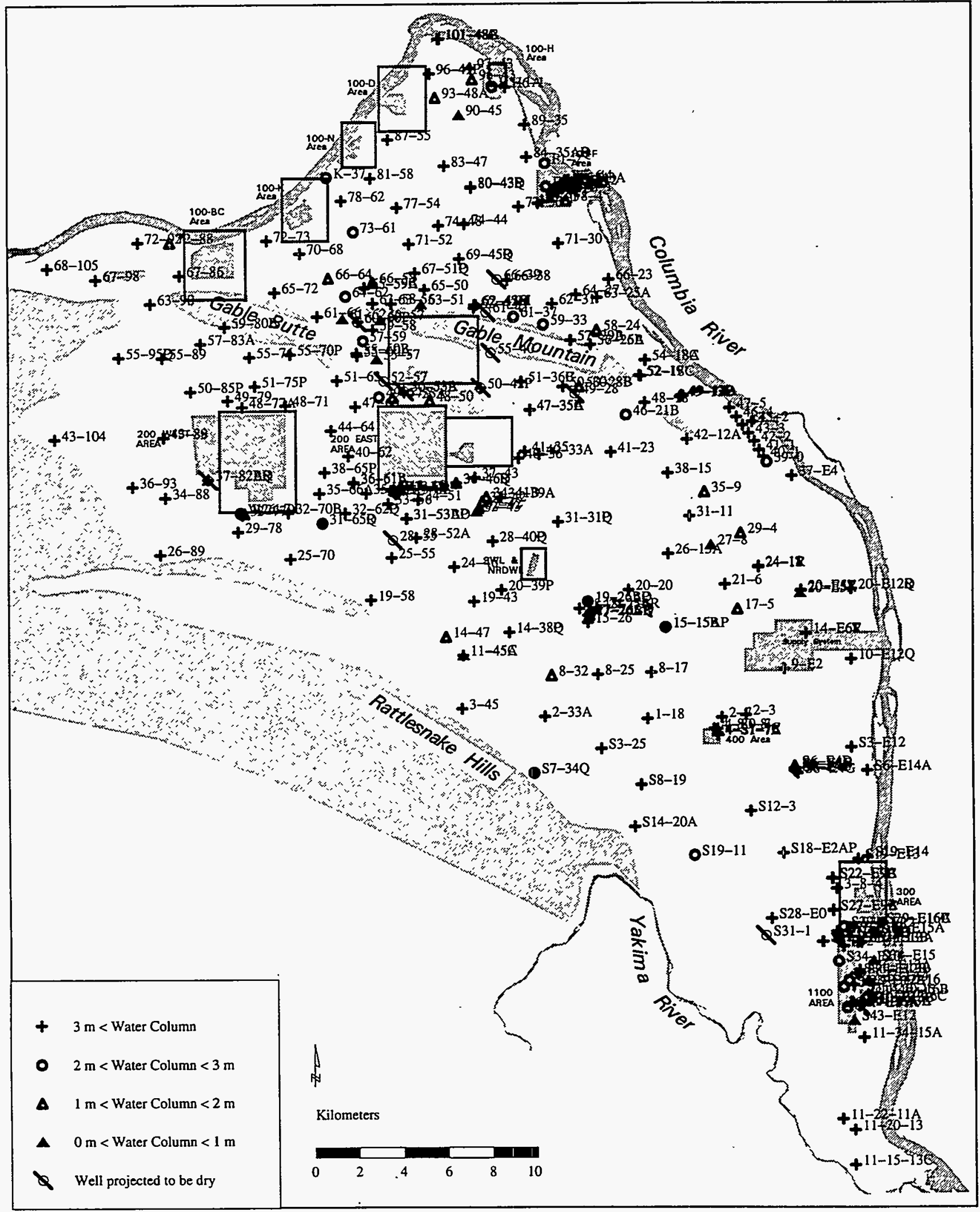

600 Area selected wells which may be impacted by declining water levels before 1 January 2000

B.2 


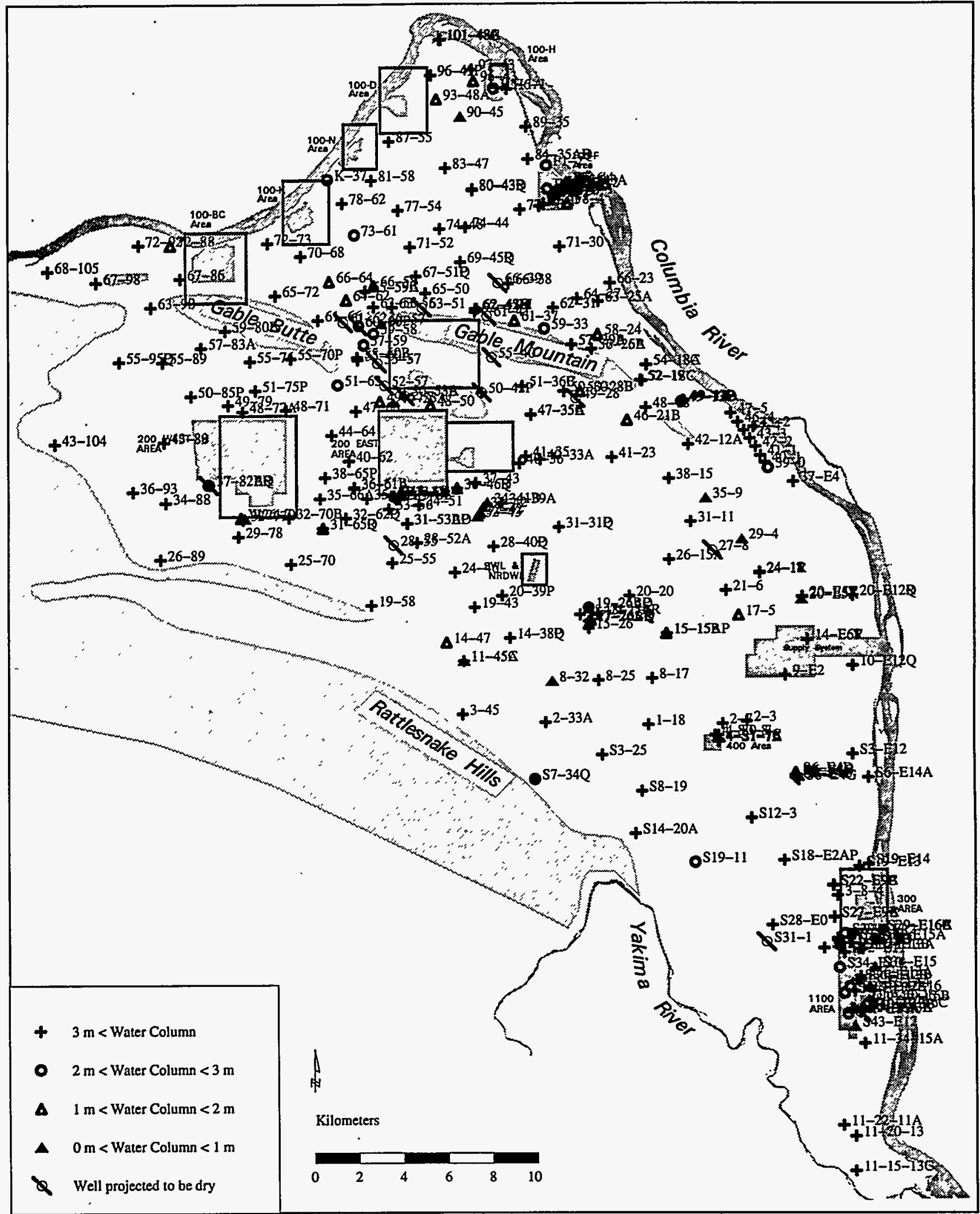

600 Area selected wells which may be impacted by declining water levels before 1 January 2005

B. 3 


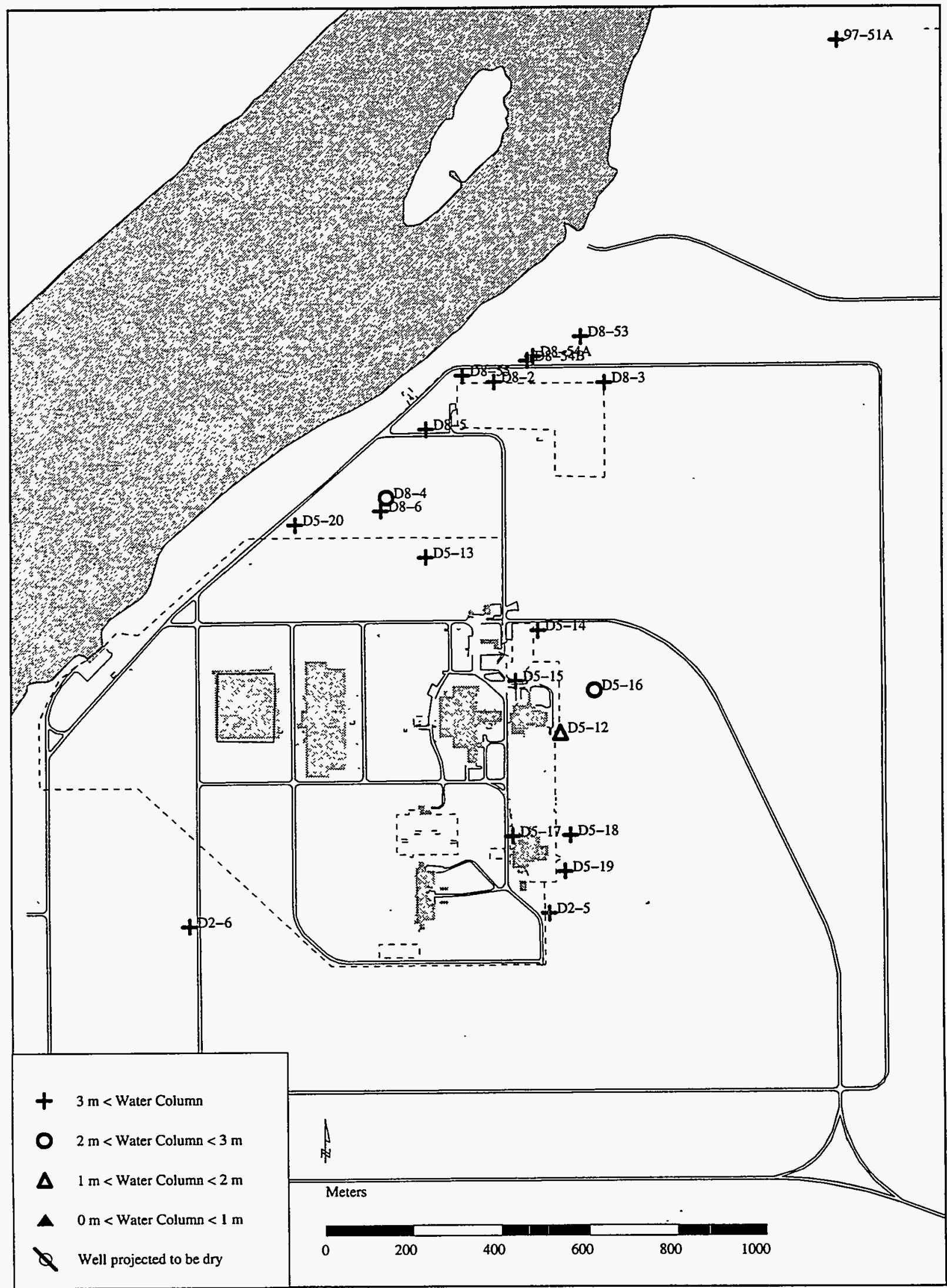

100-D Area selected wells which may be impacted by declining water levels before 1 January 1995 
$S^{\circ} \cdot \mathrm{g}$

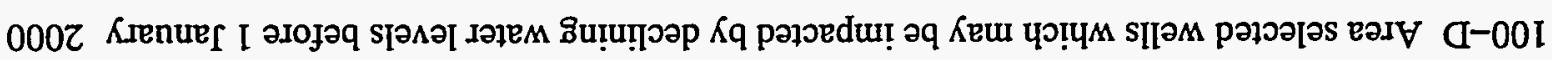

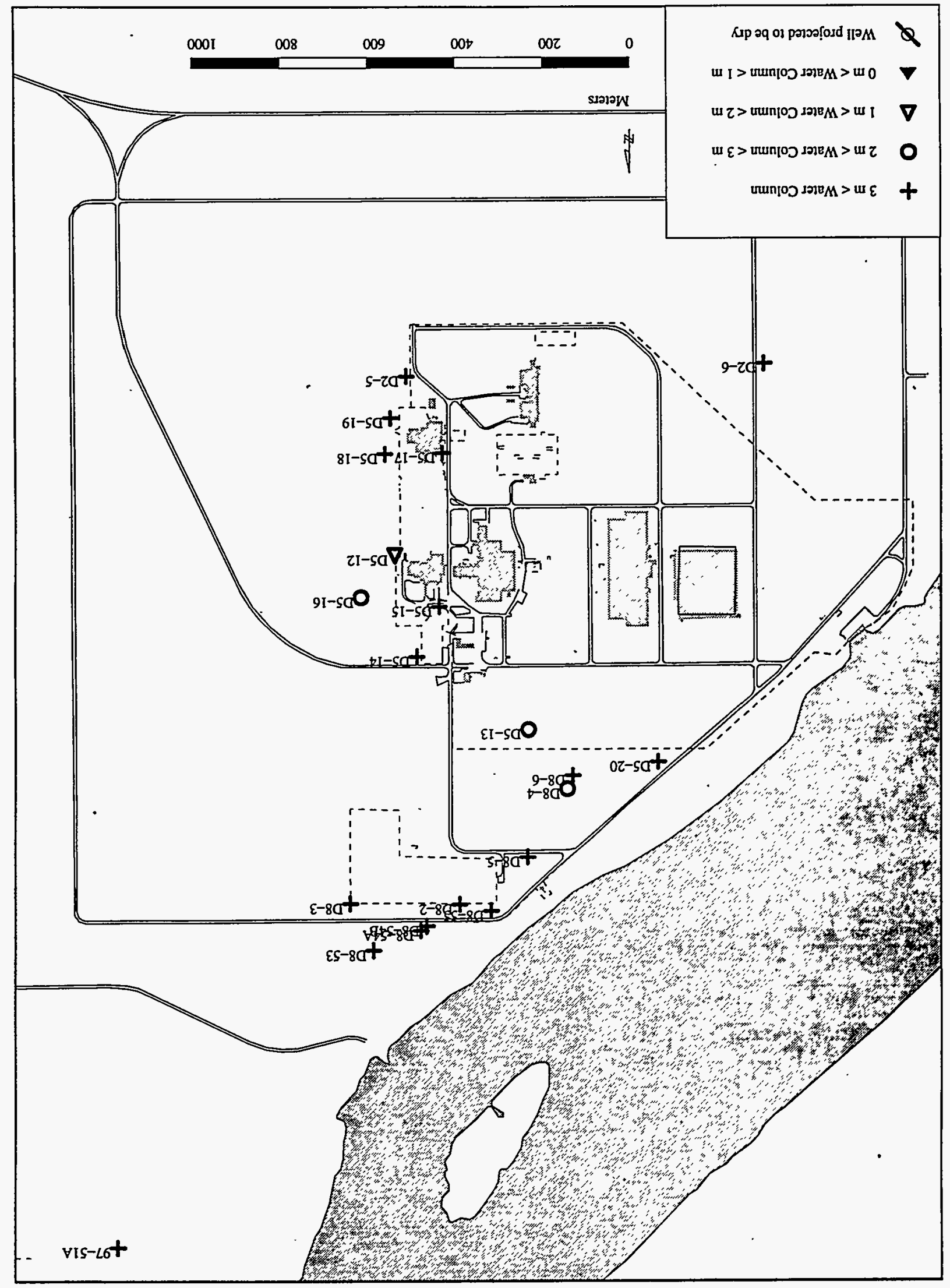




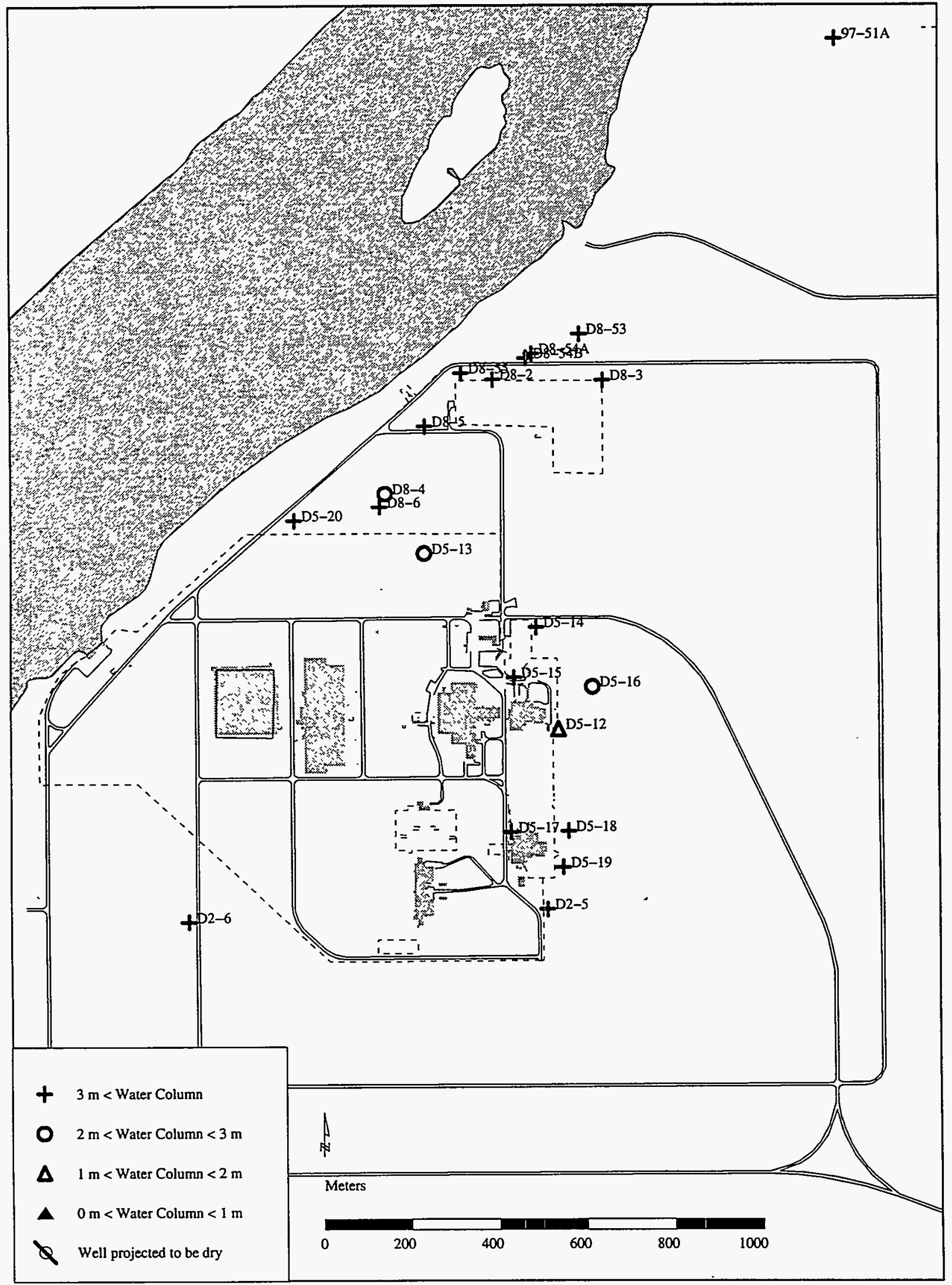

100-D Area selected wells which may be impacted by declining water levels before 1 January 2005

B. 6 
$L^{\circ} \mathrm{g}$

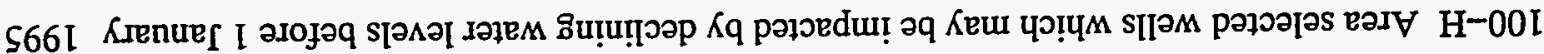

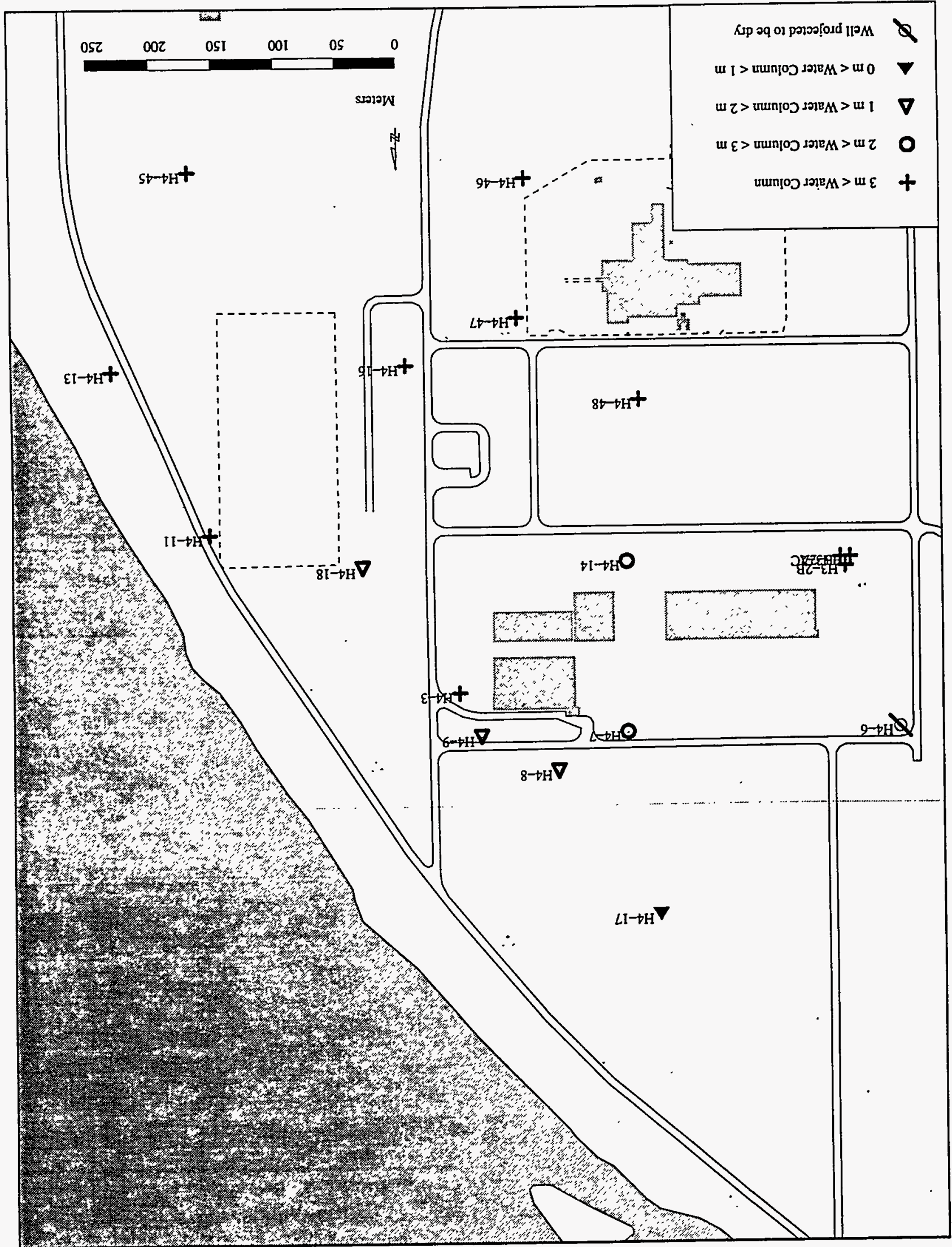




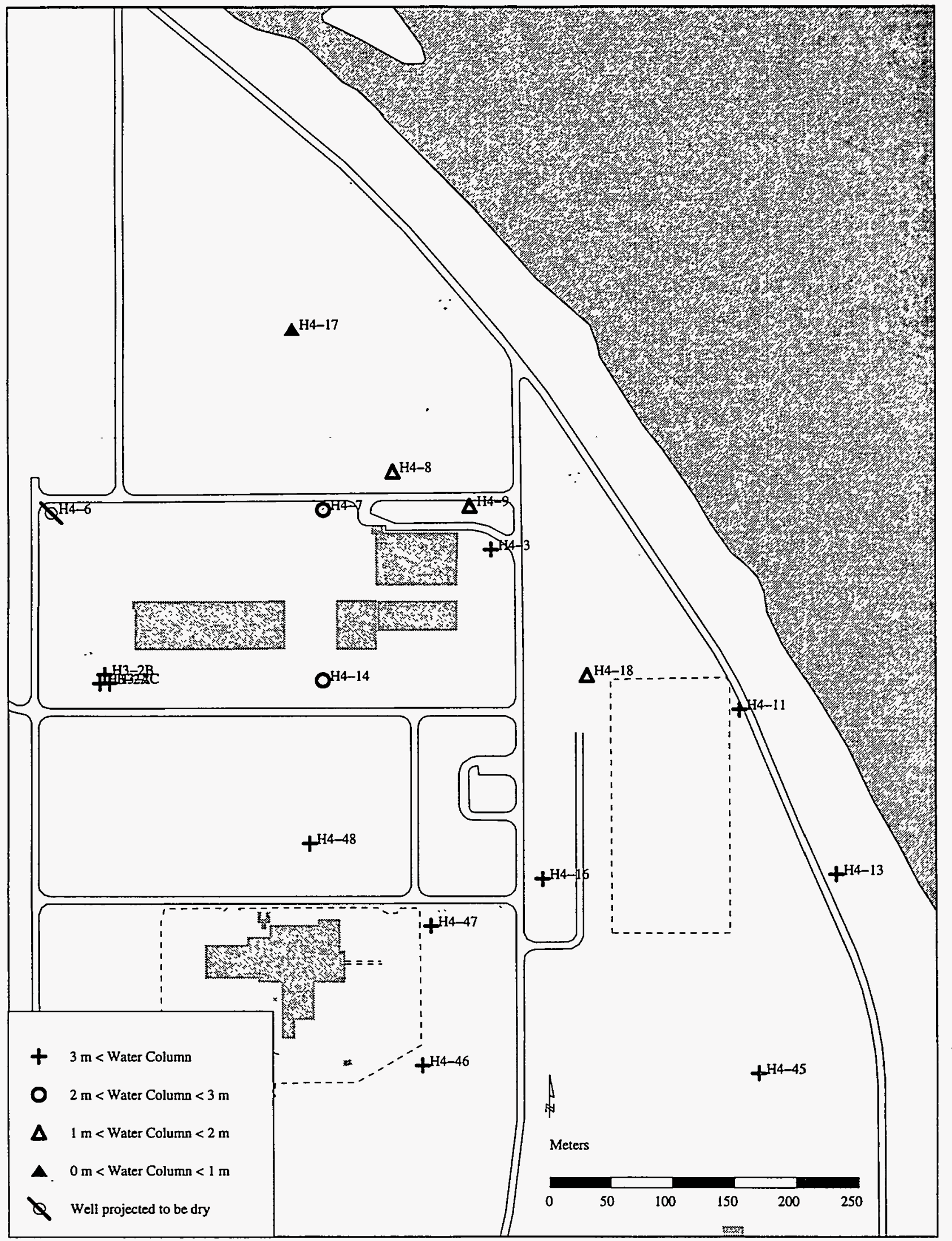

100-H Area selected wells which may be impacted by declining water levels before 1 January 2000

B. 8 


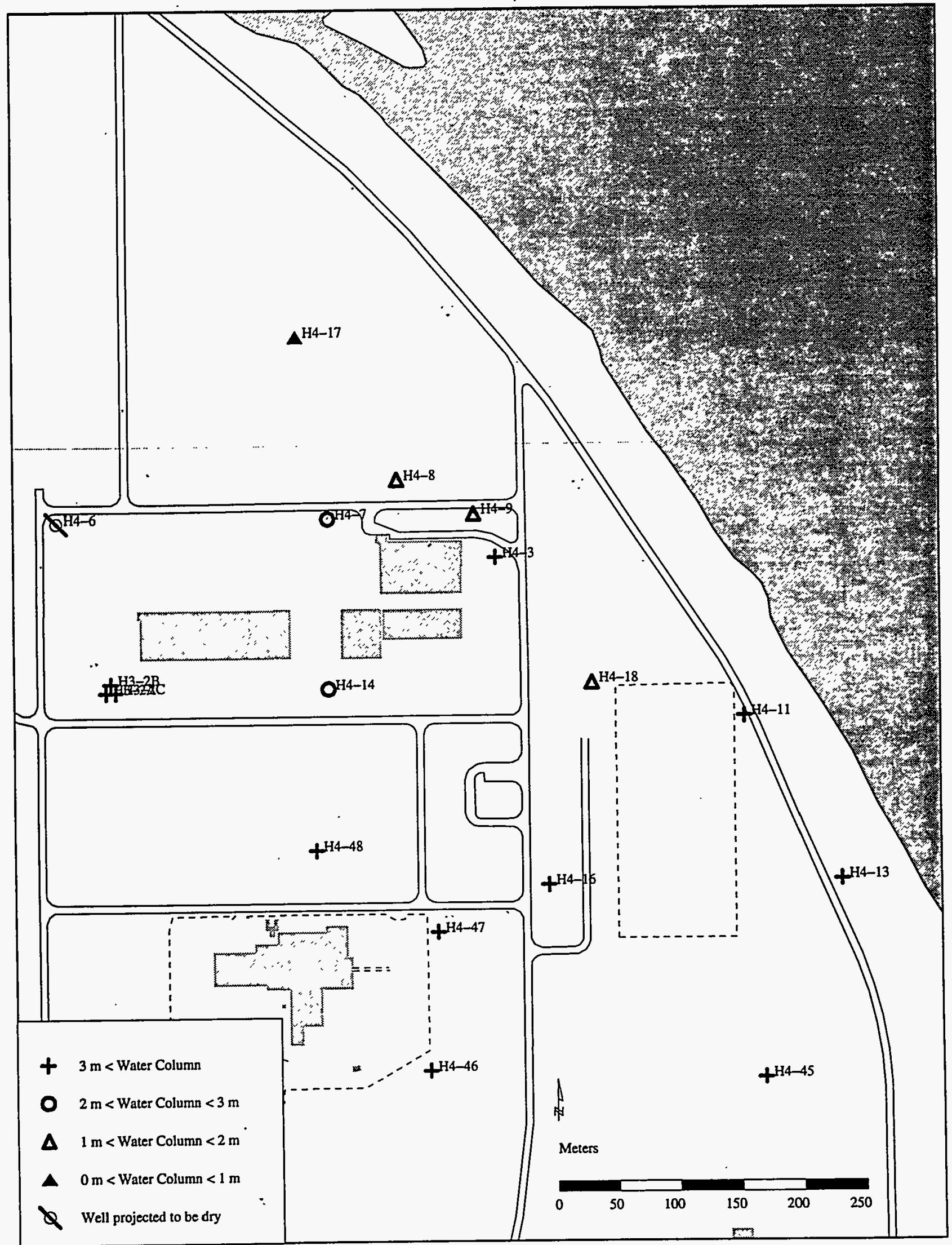

100-H Area selected wells which may be impacted by declining water levels before 1 January 2005 


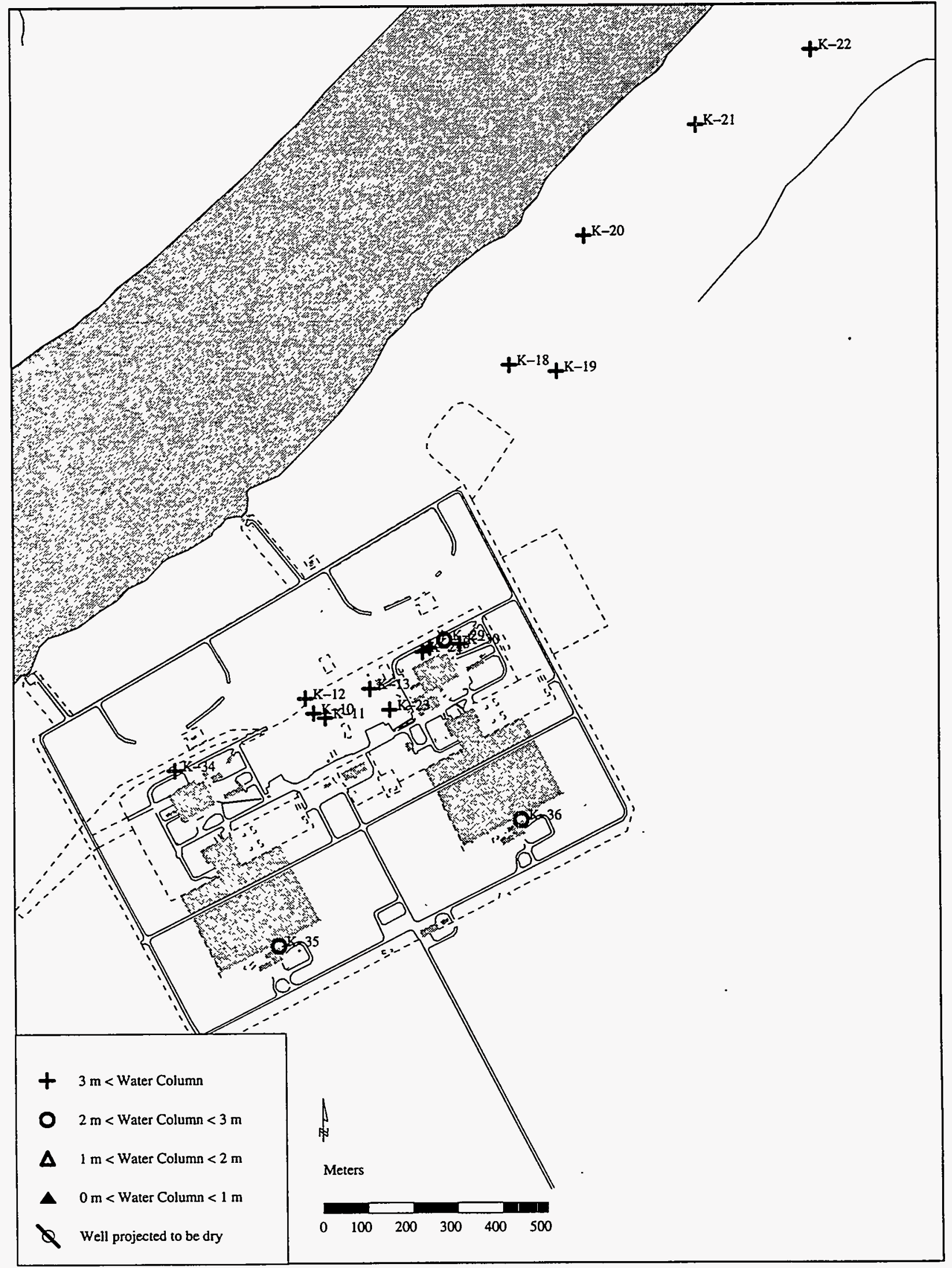

100-K Area selected wells which may be impacted by declining water levels before 1 January 1995

B. 10 
II'G

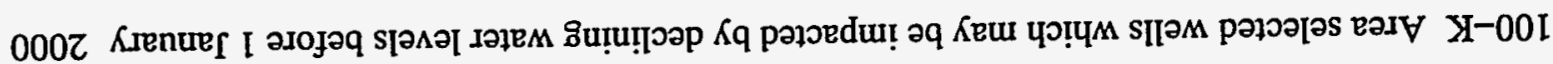

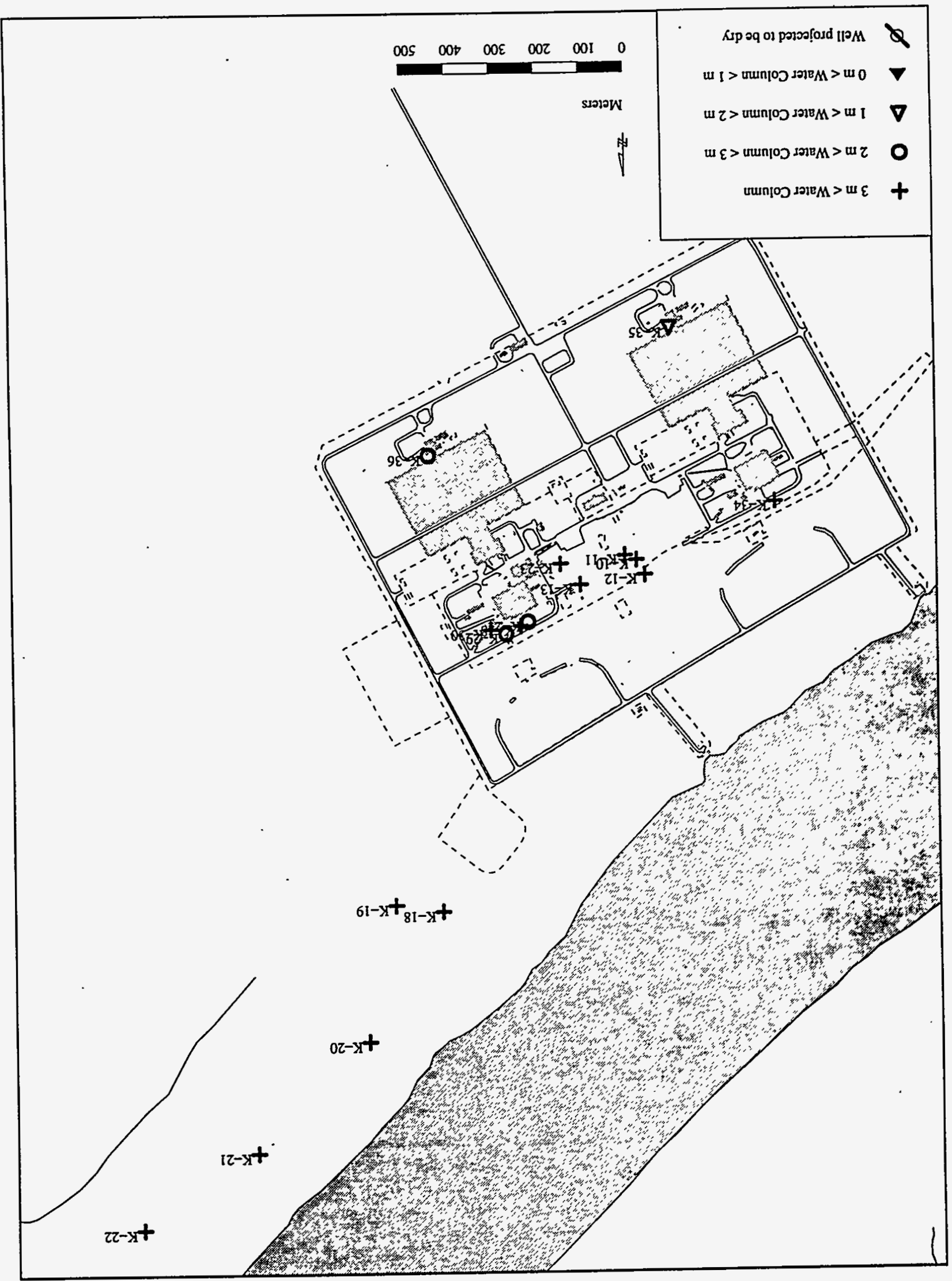




\section{ZI'G}

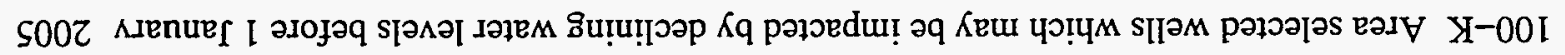

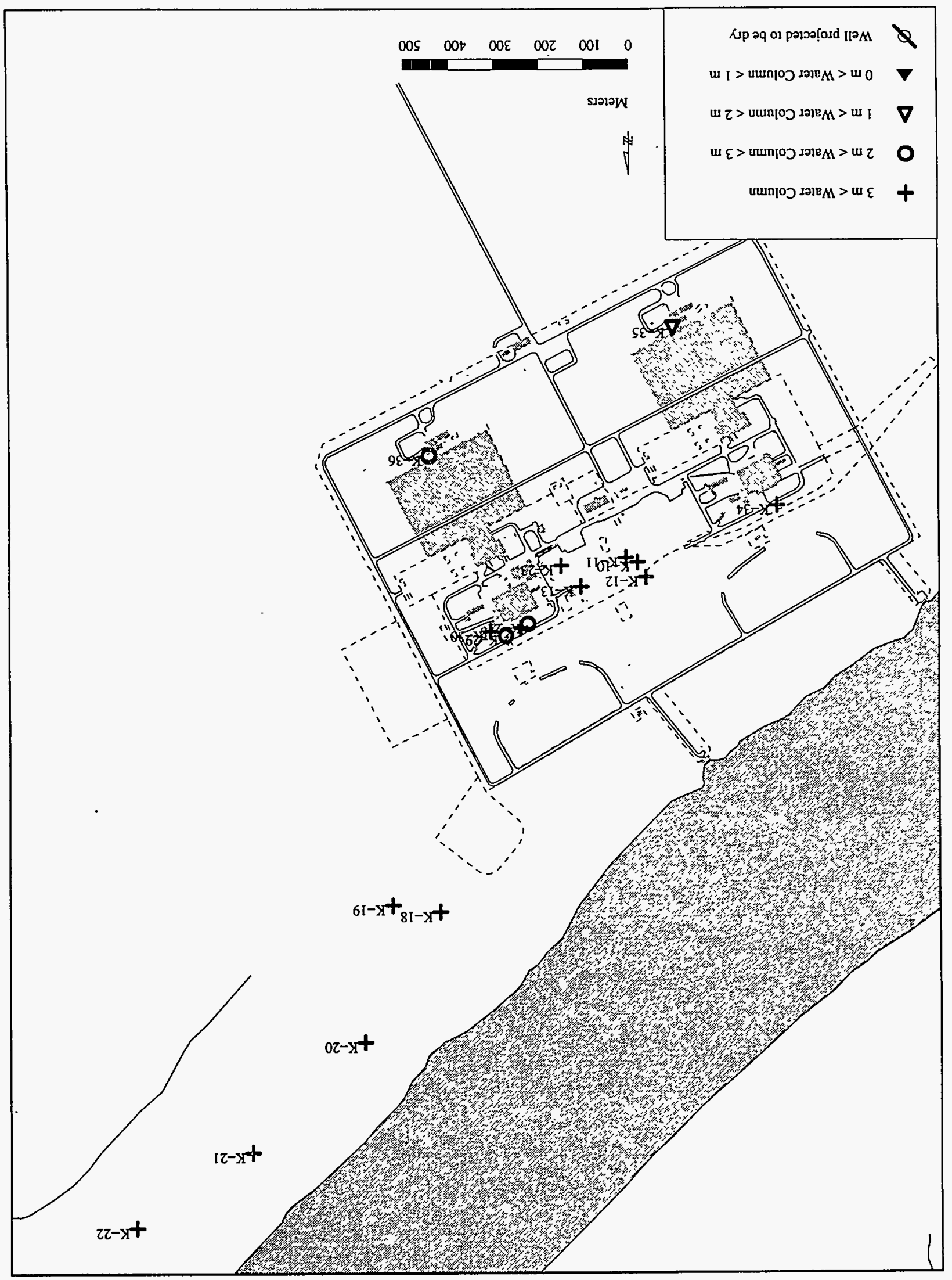




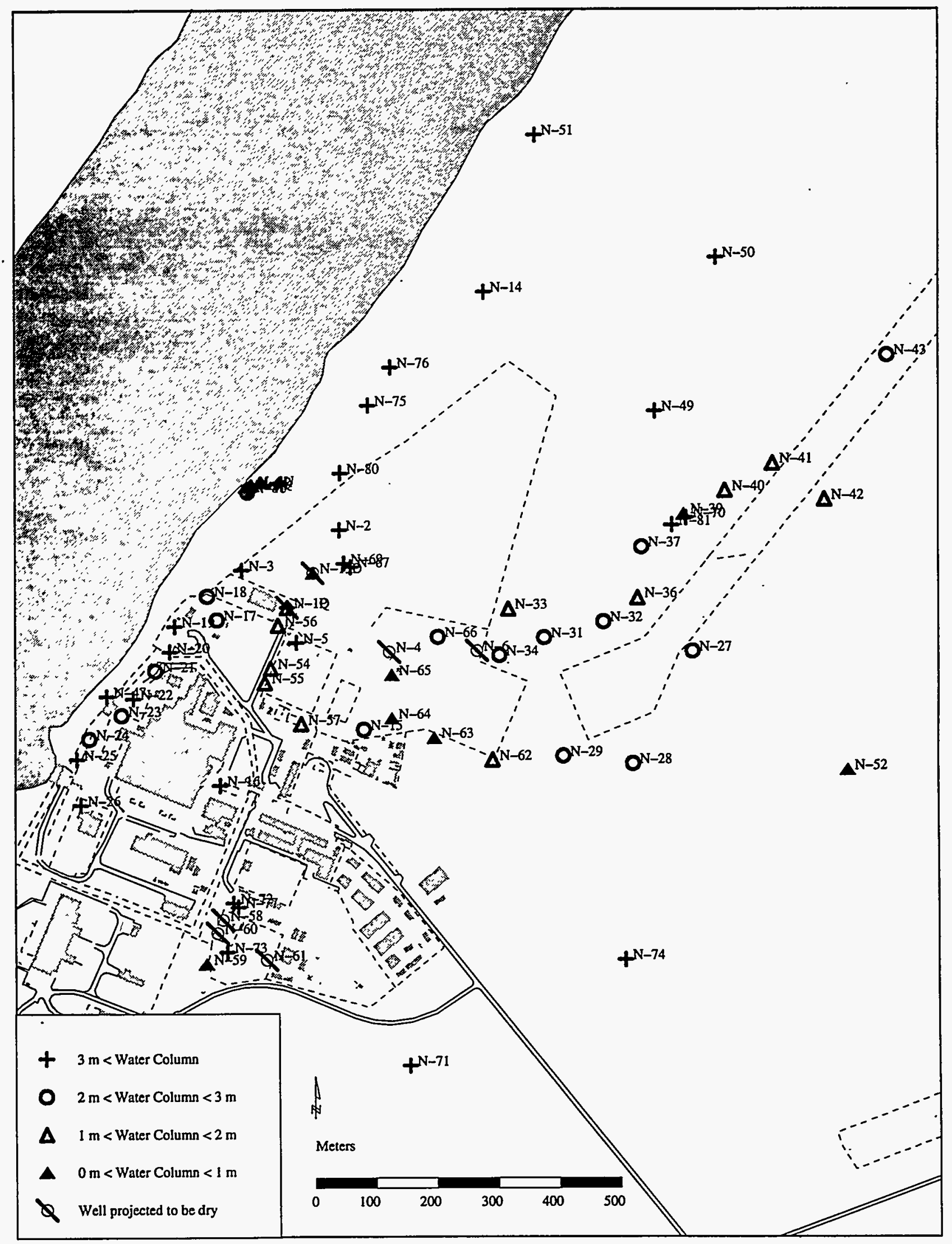

100-N Area selected wells which may be impacted by declining water levels before 1 January 1995 


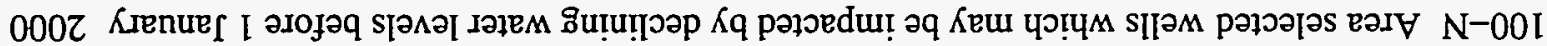

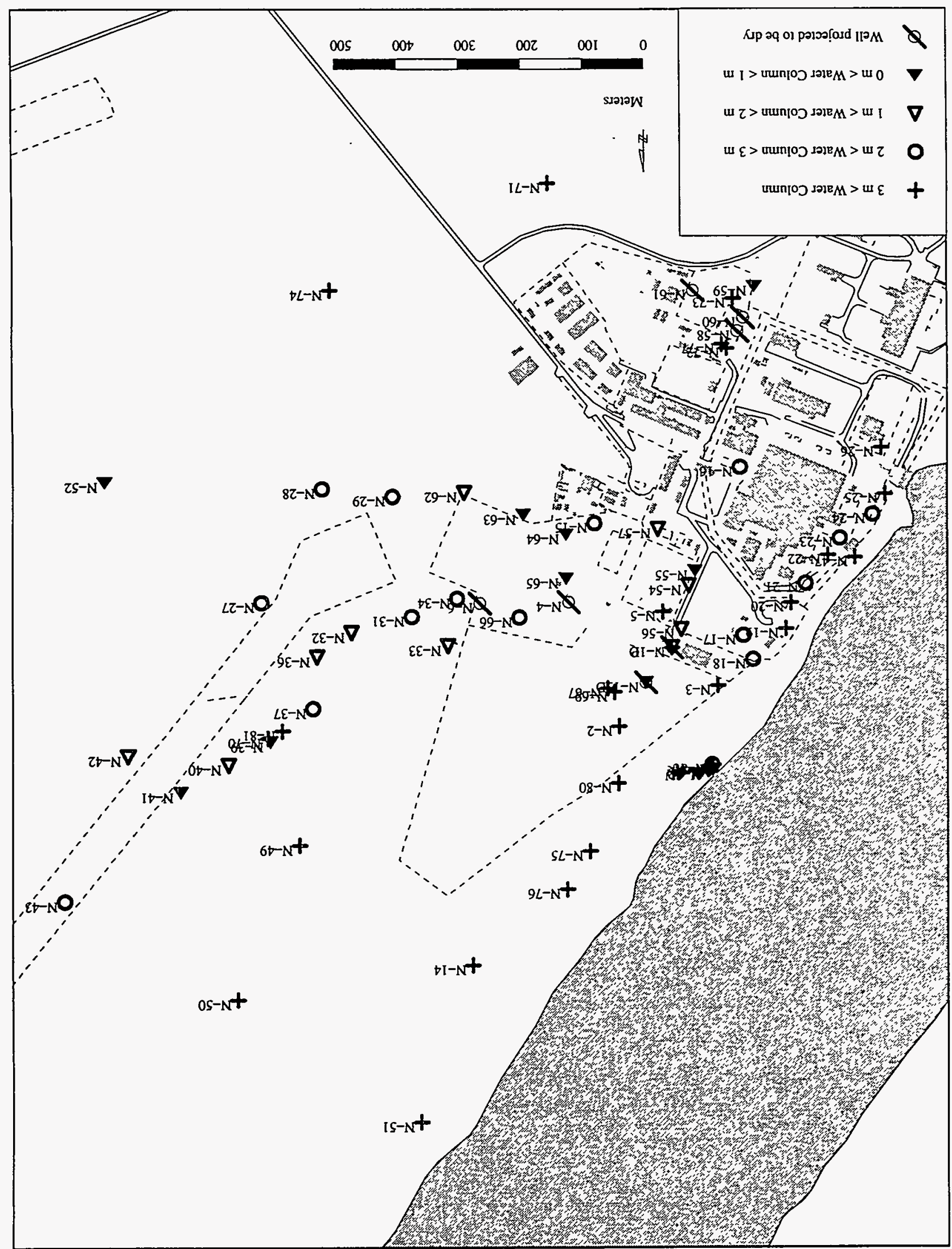




\section{sI'g}

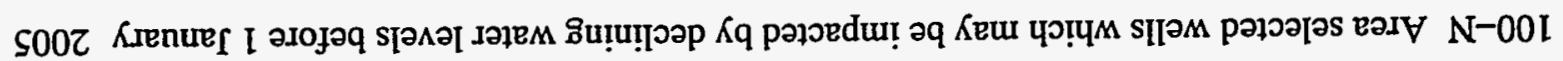

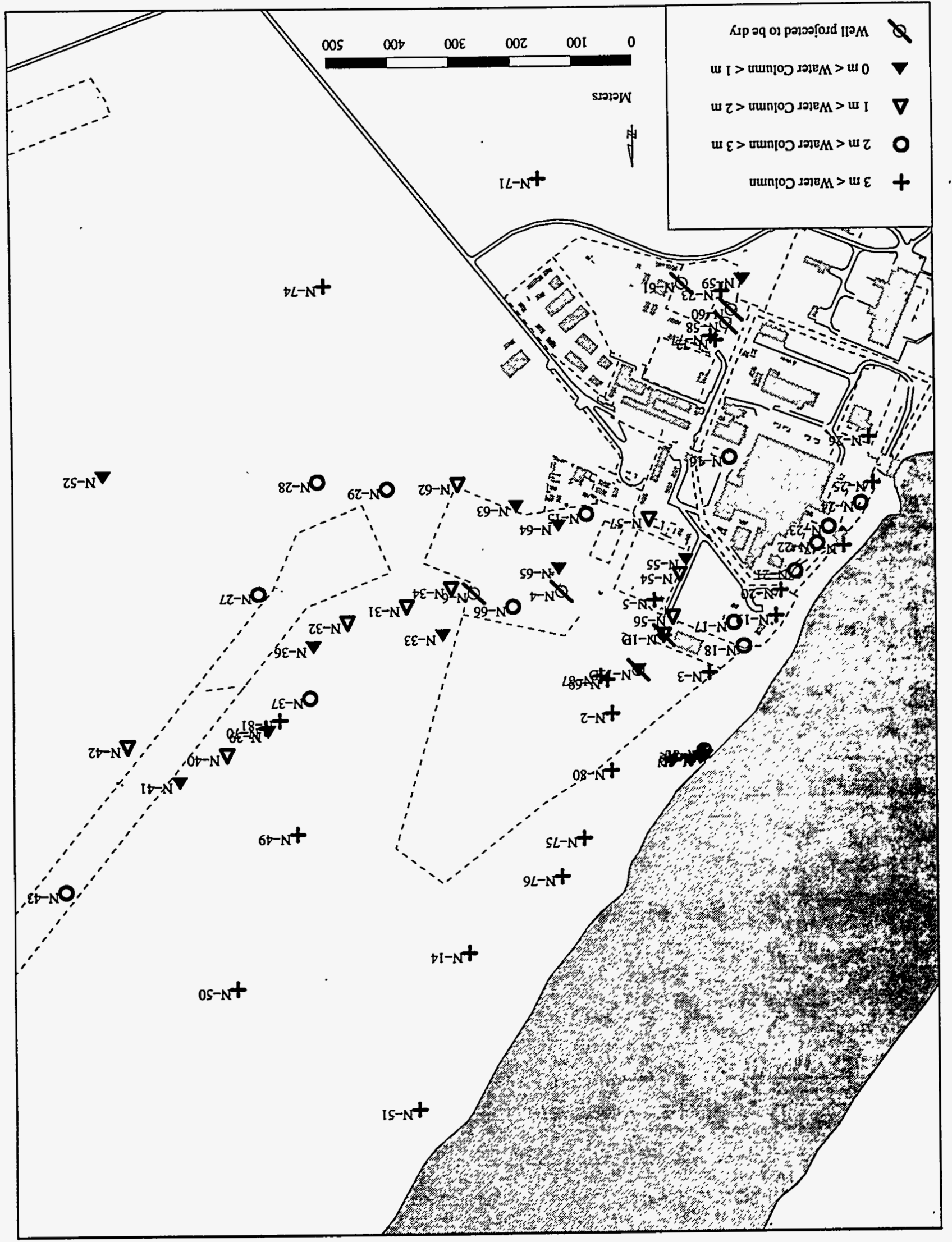




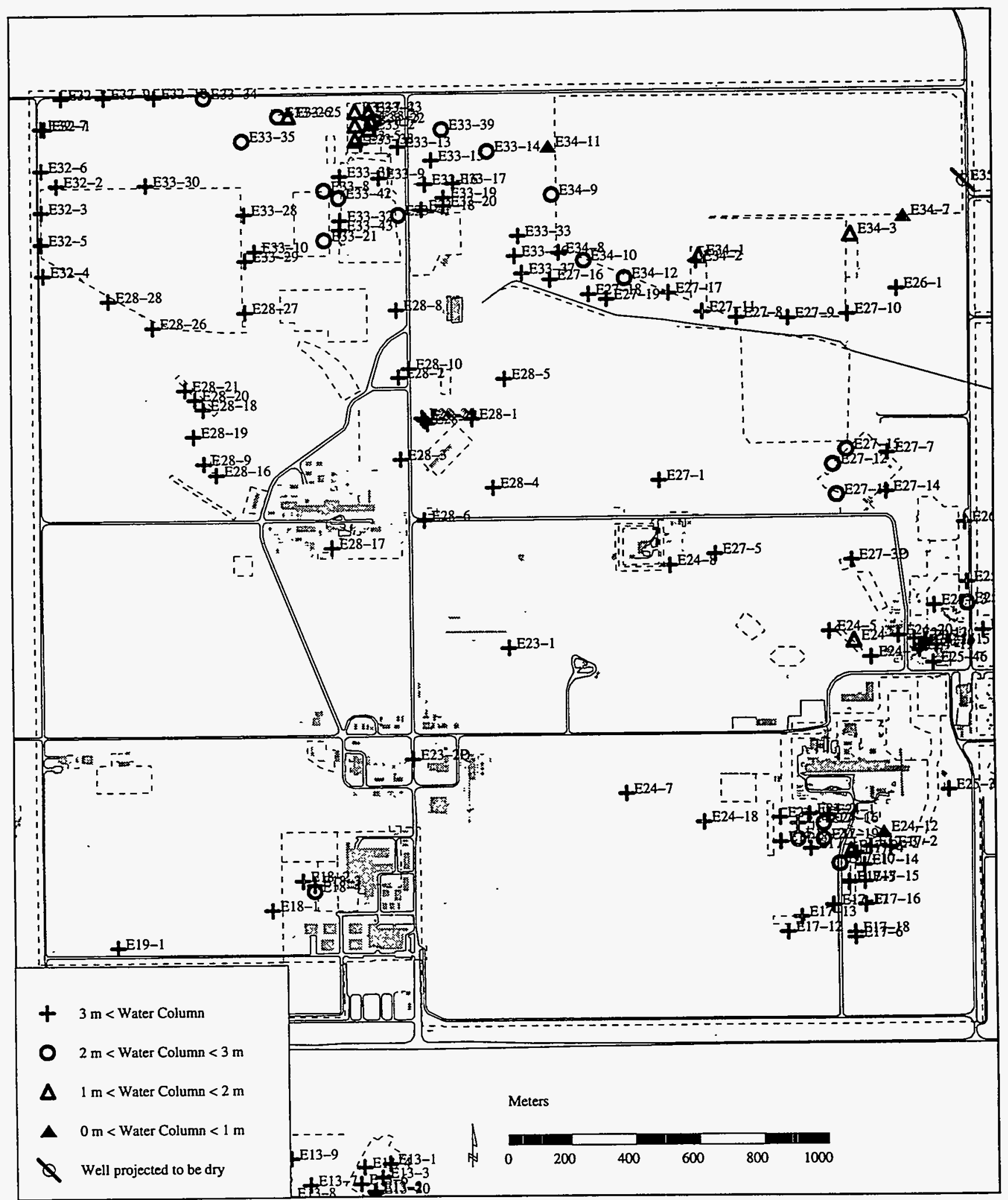

200-East Area selected wells which may be impacted by declining water levels before 1 January 1995

B. 16 


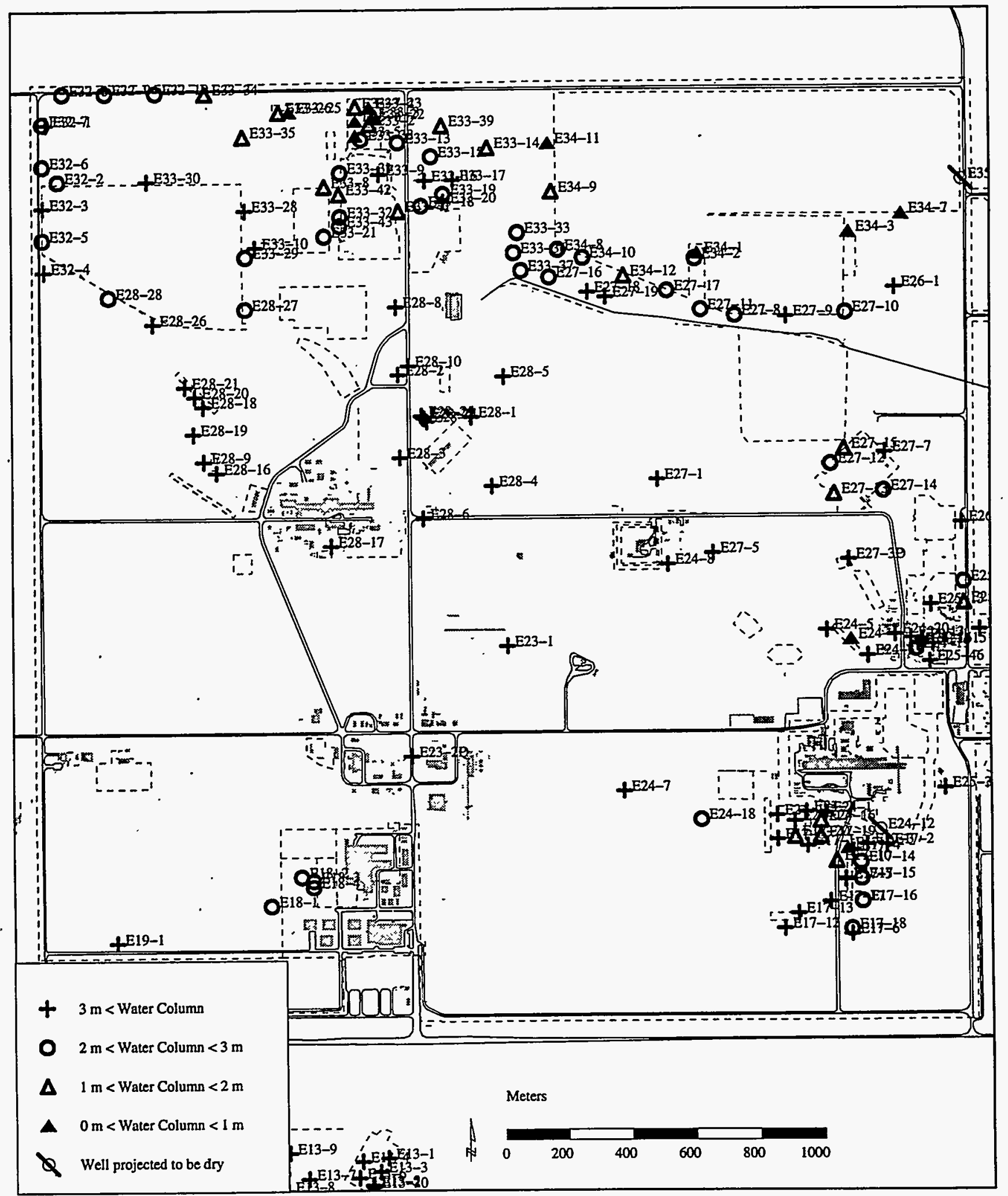

200-East Area selected wells which may be impacted by declining water levels before 1 January 2000 


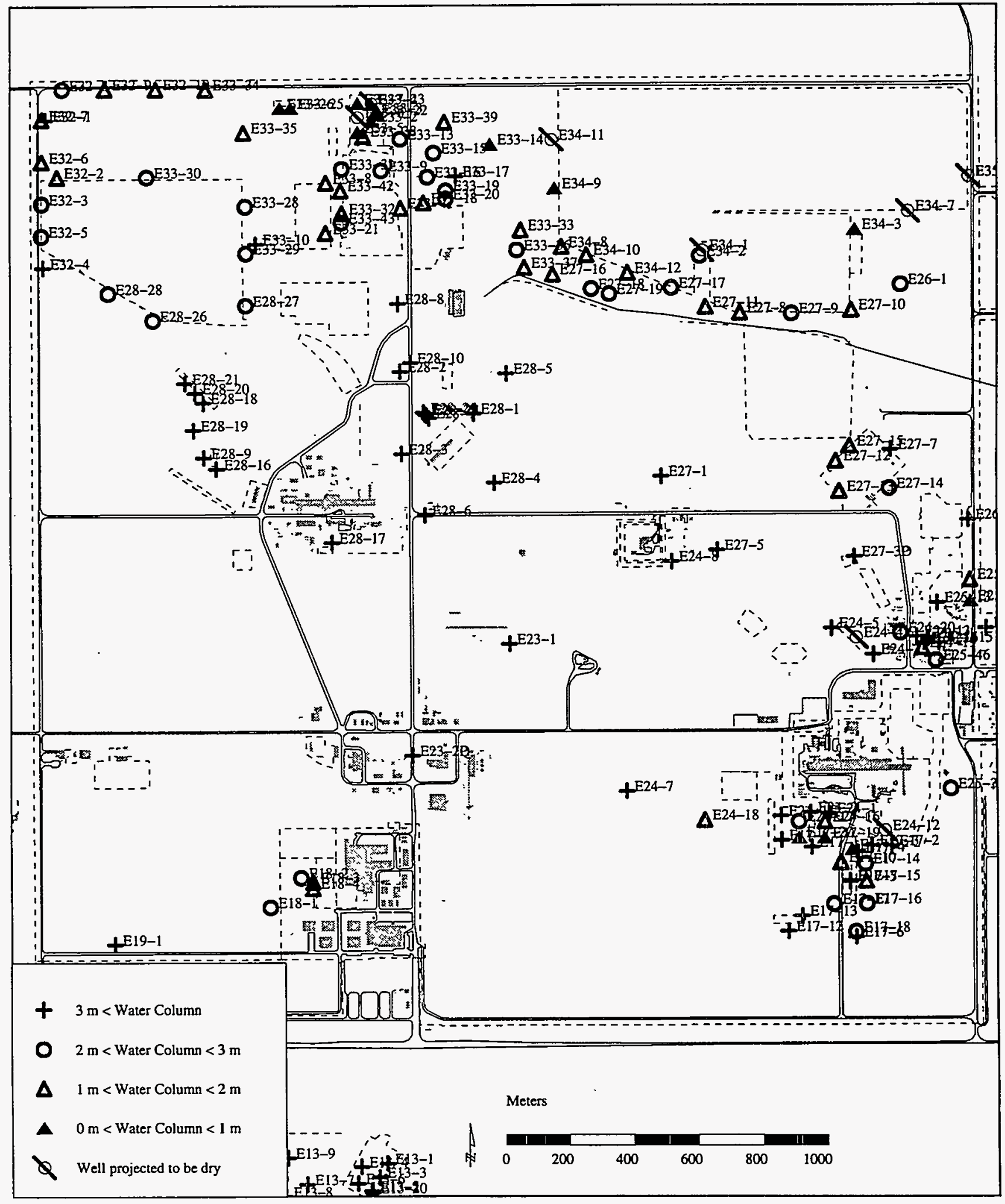

200-East Area selected wells which may be impacted by declining water levels before 1 January 2005 


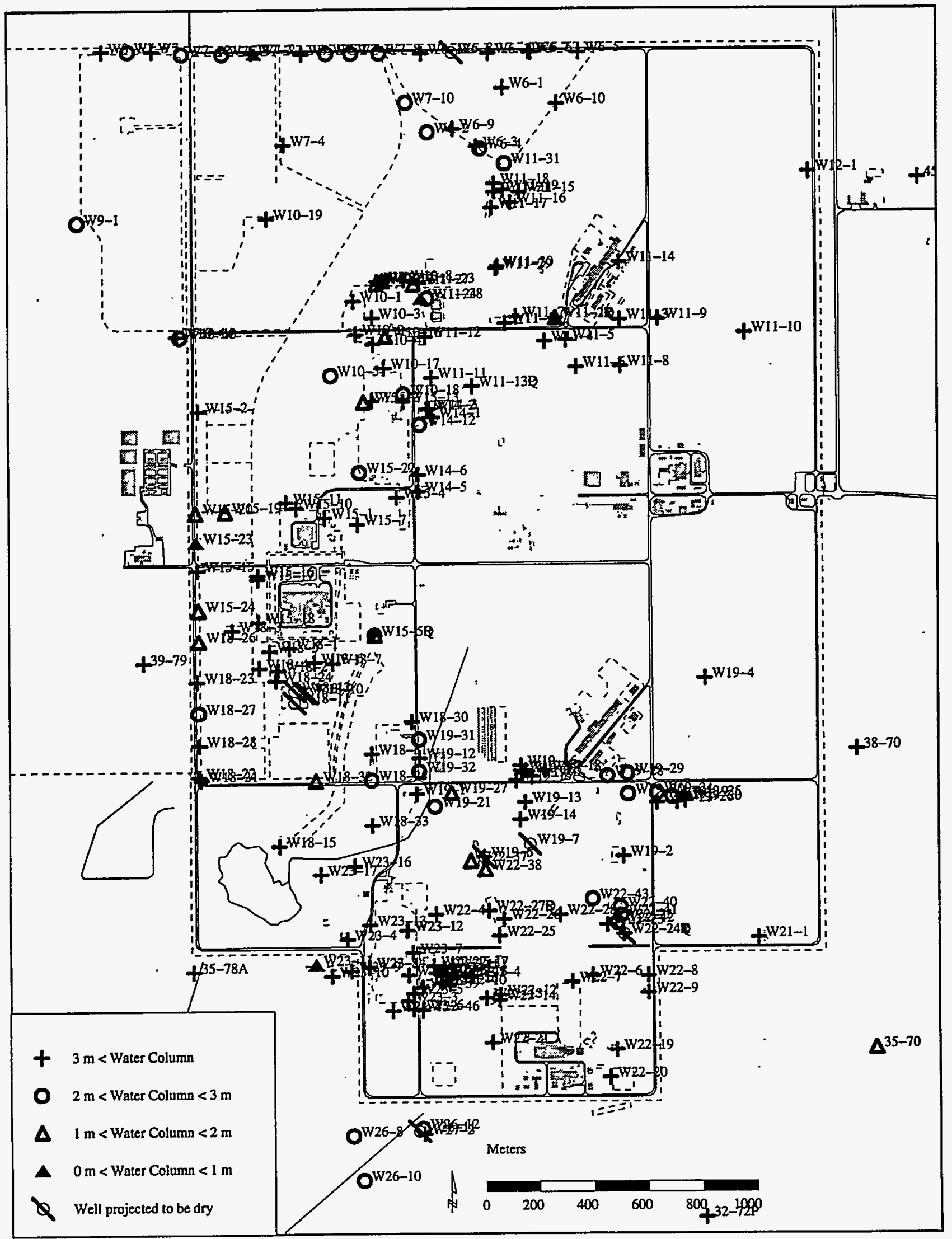

200-West Area selected wells which may be impacted by declining water levels before 1 January 1995 


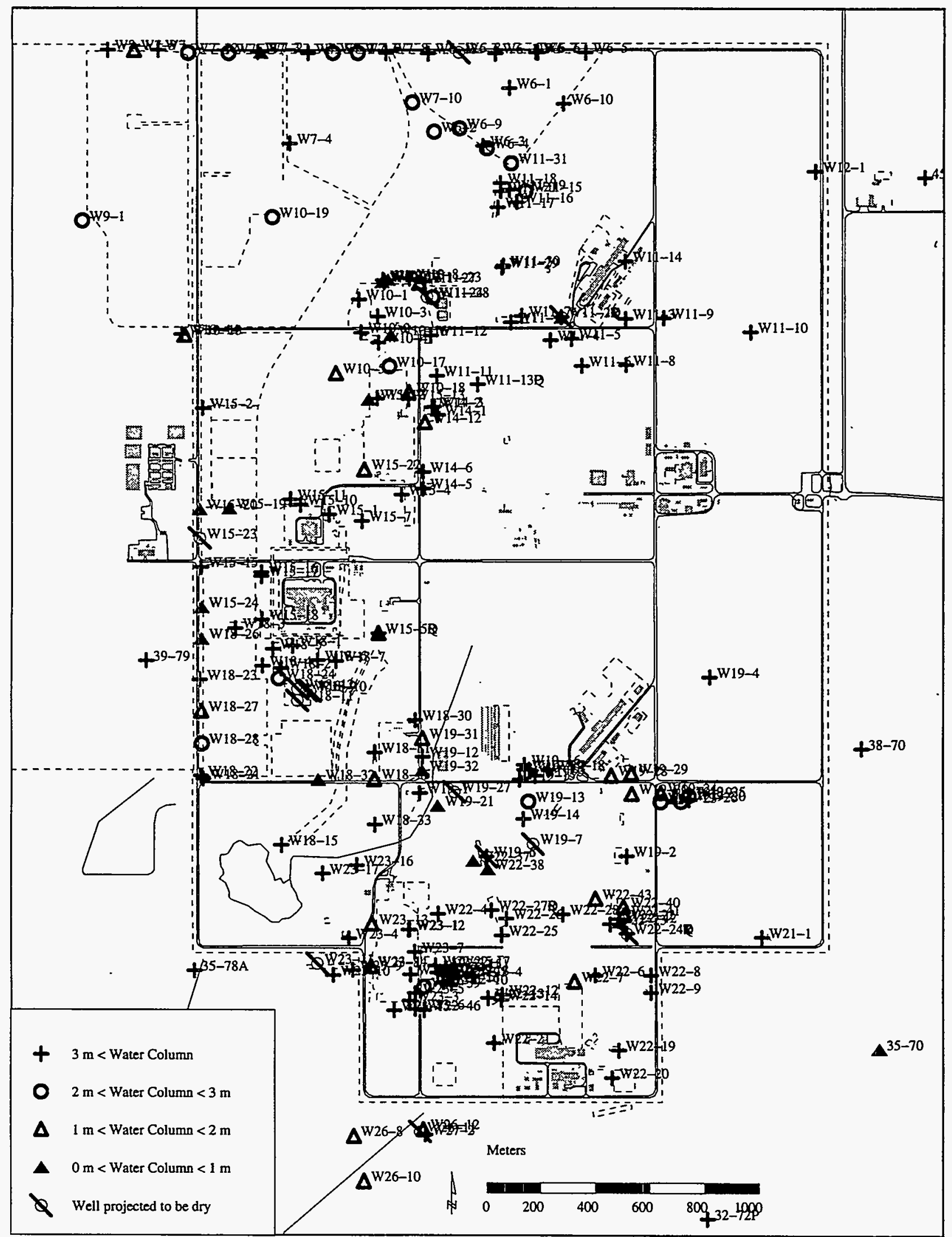

200-West Area selected wells which may be impacted by declining water levels before 1 Januarv 2000 


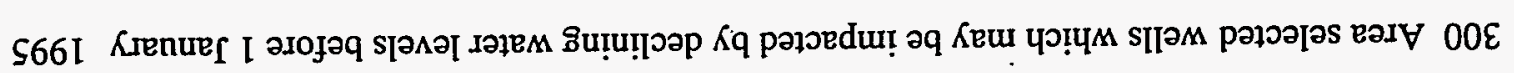

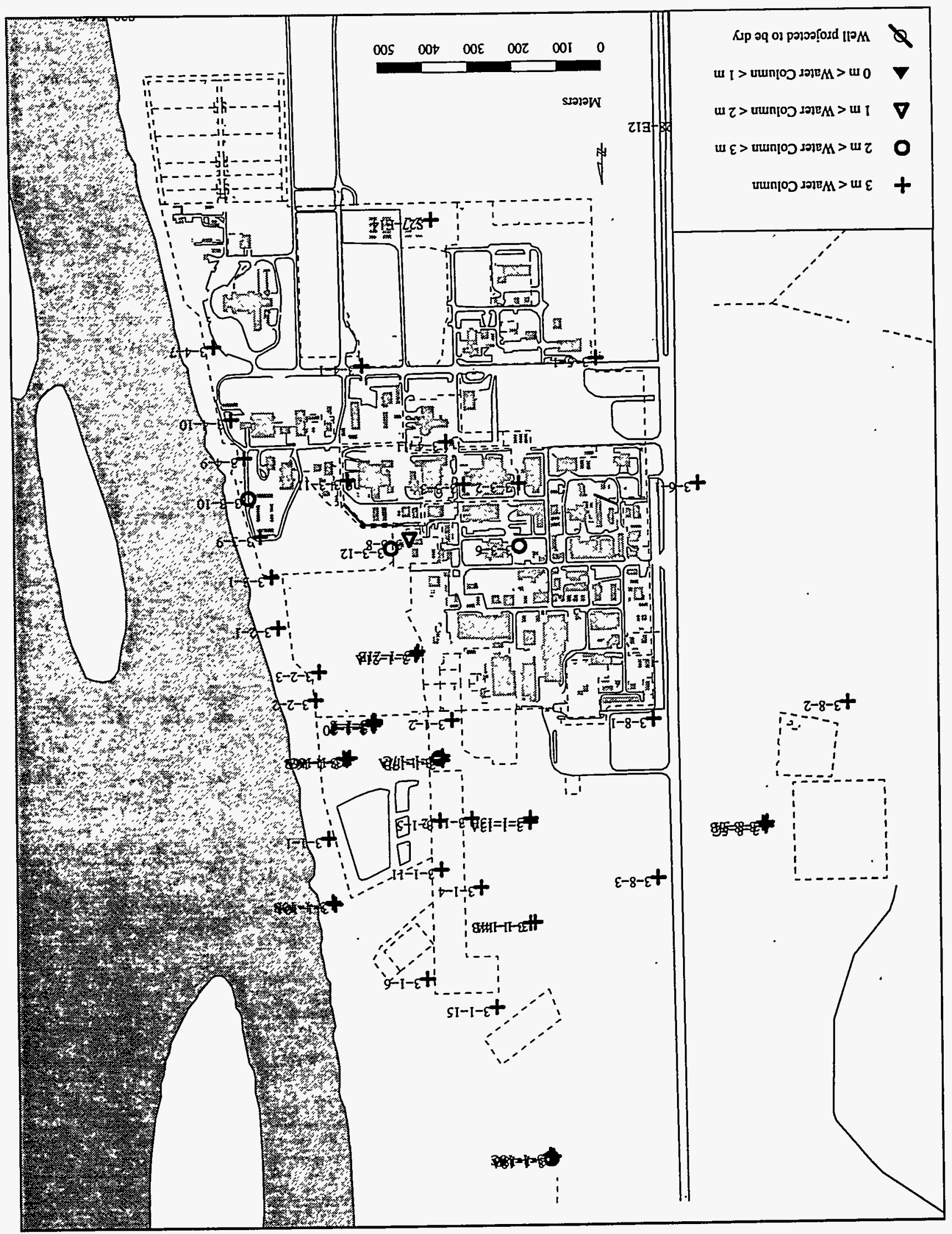




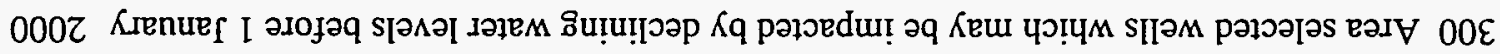

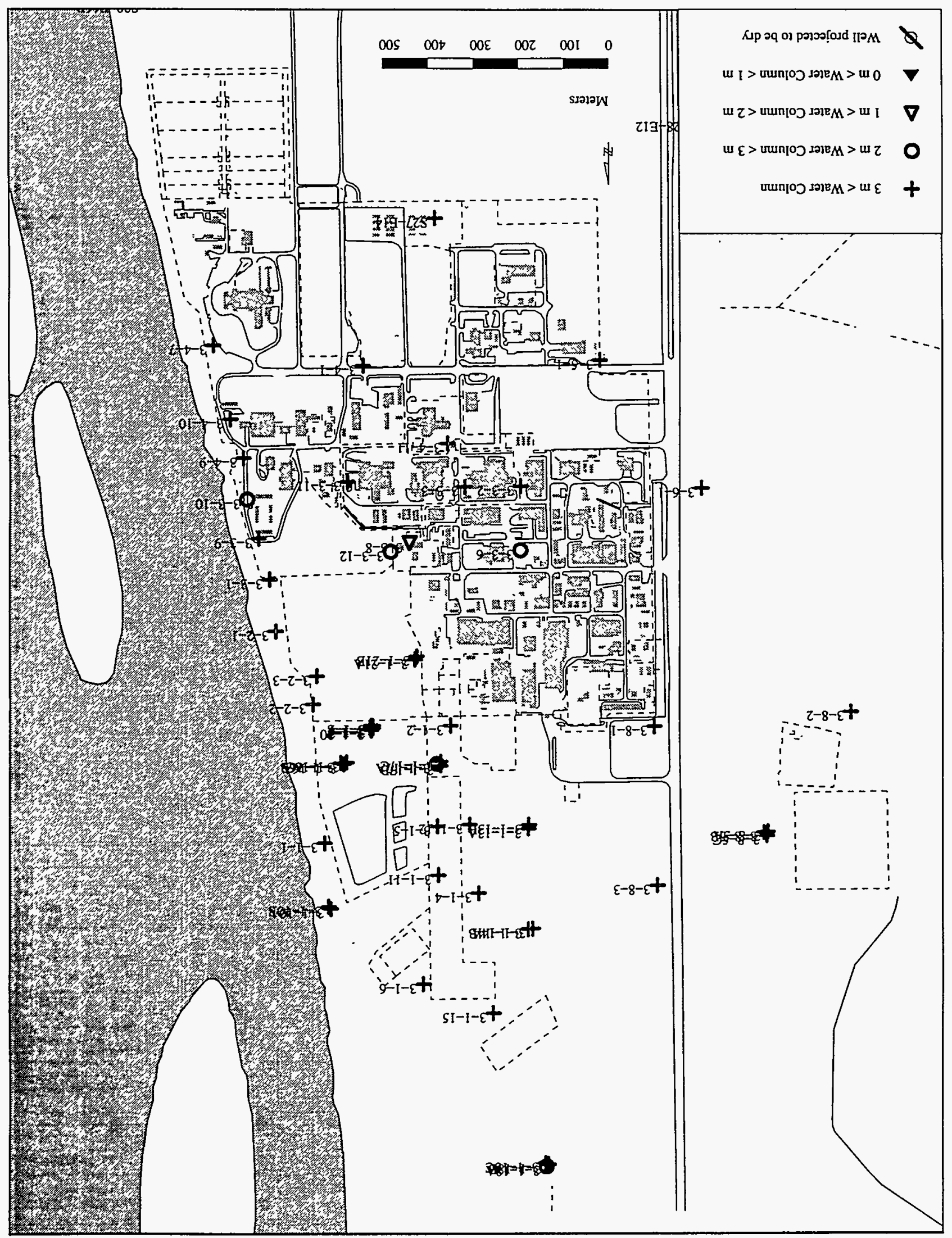




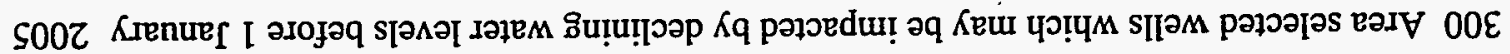

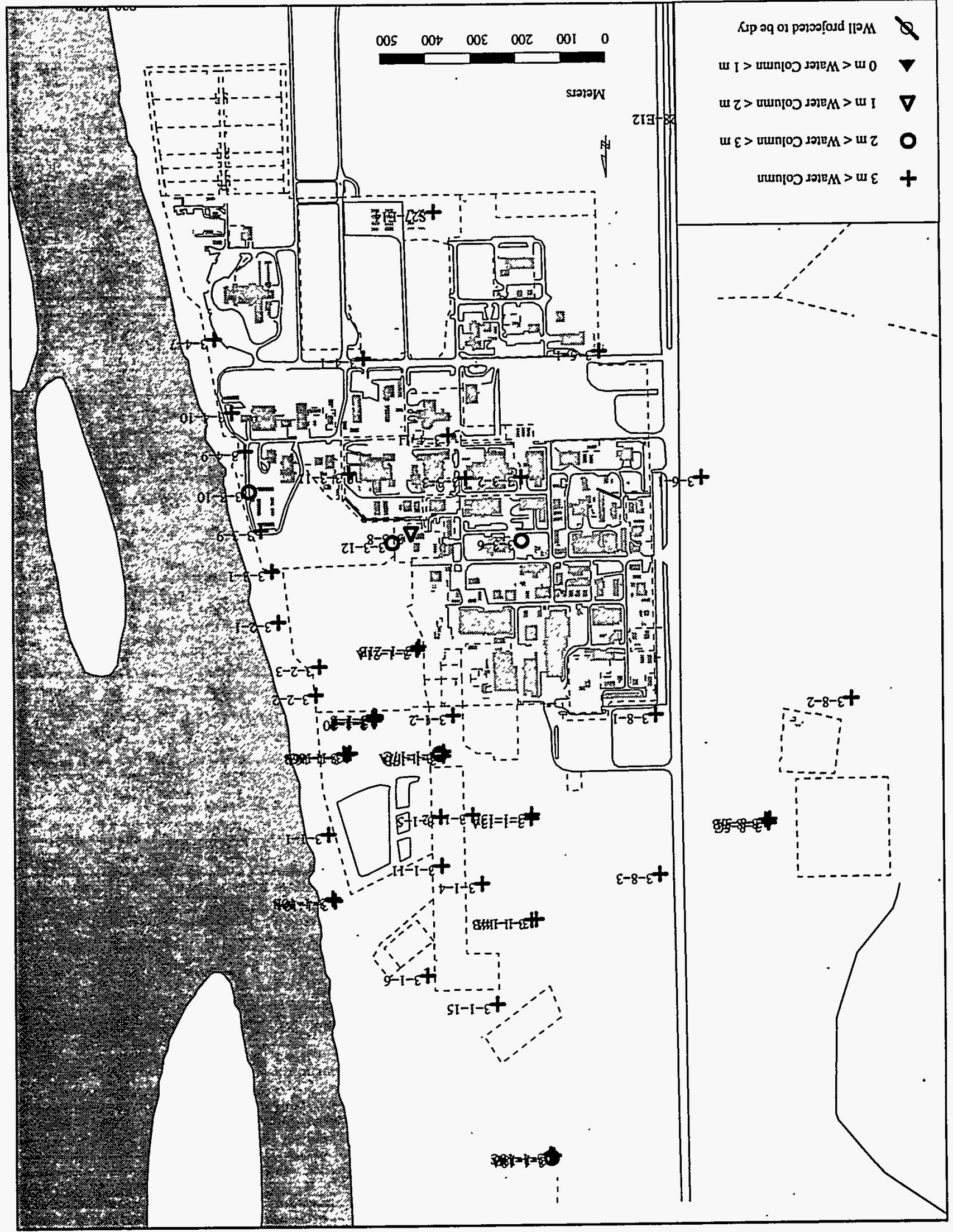




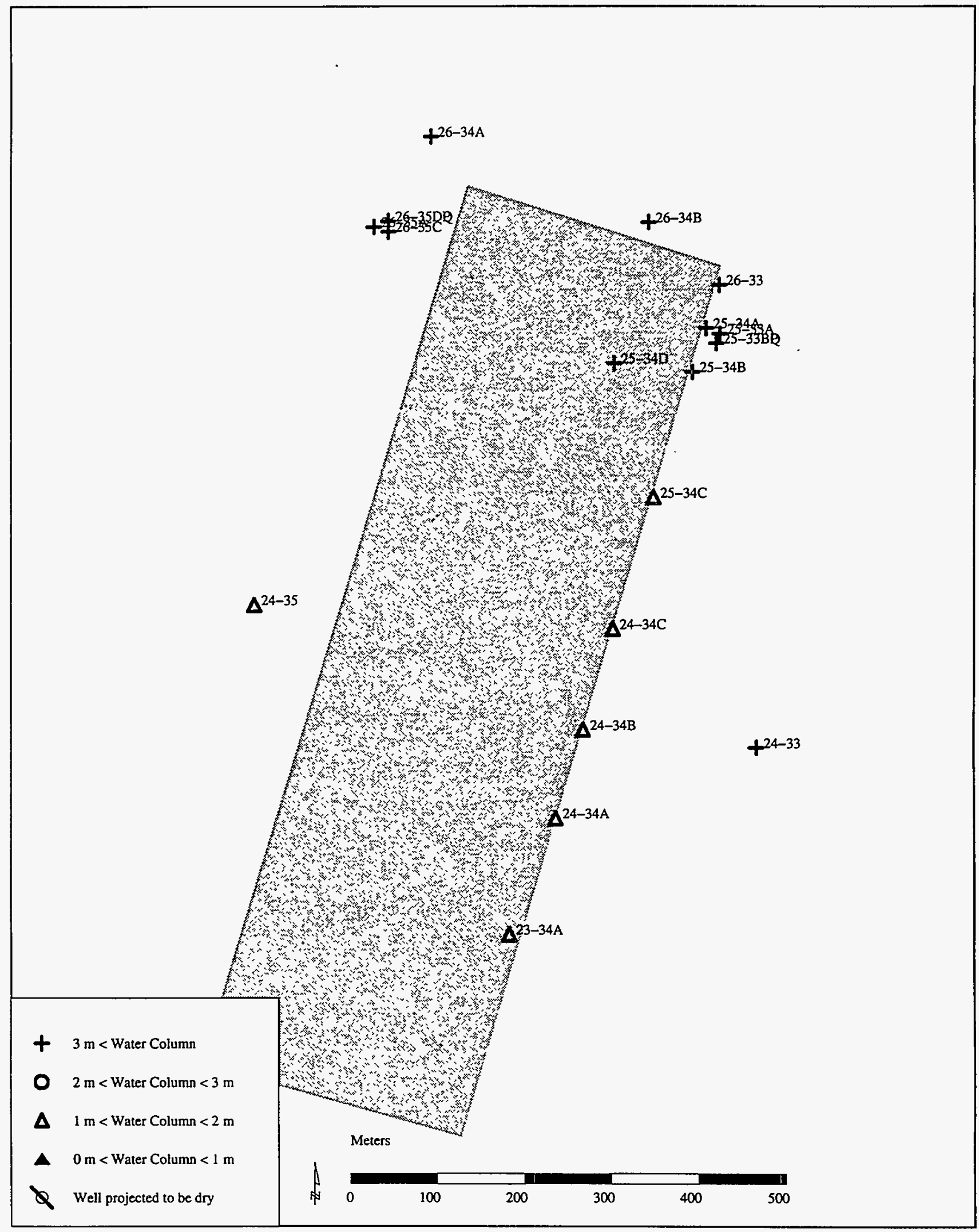

SWL_\&_NRDWL Area selected wells which may be impacted by declining water levels before 1 January 1995 


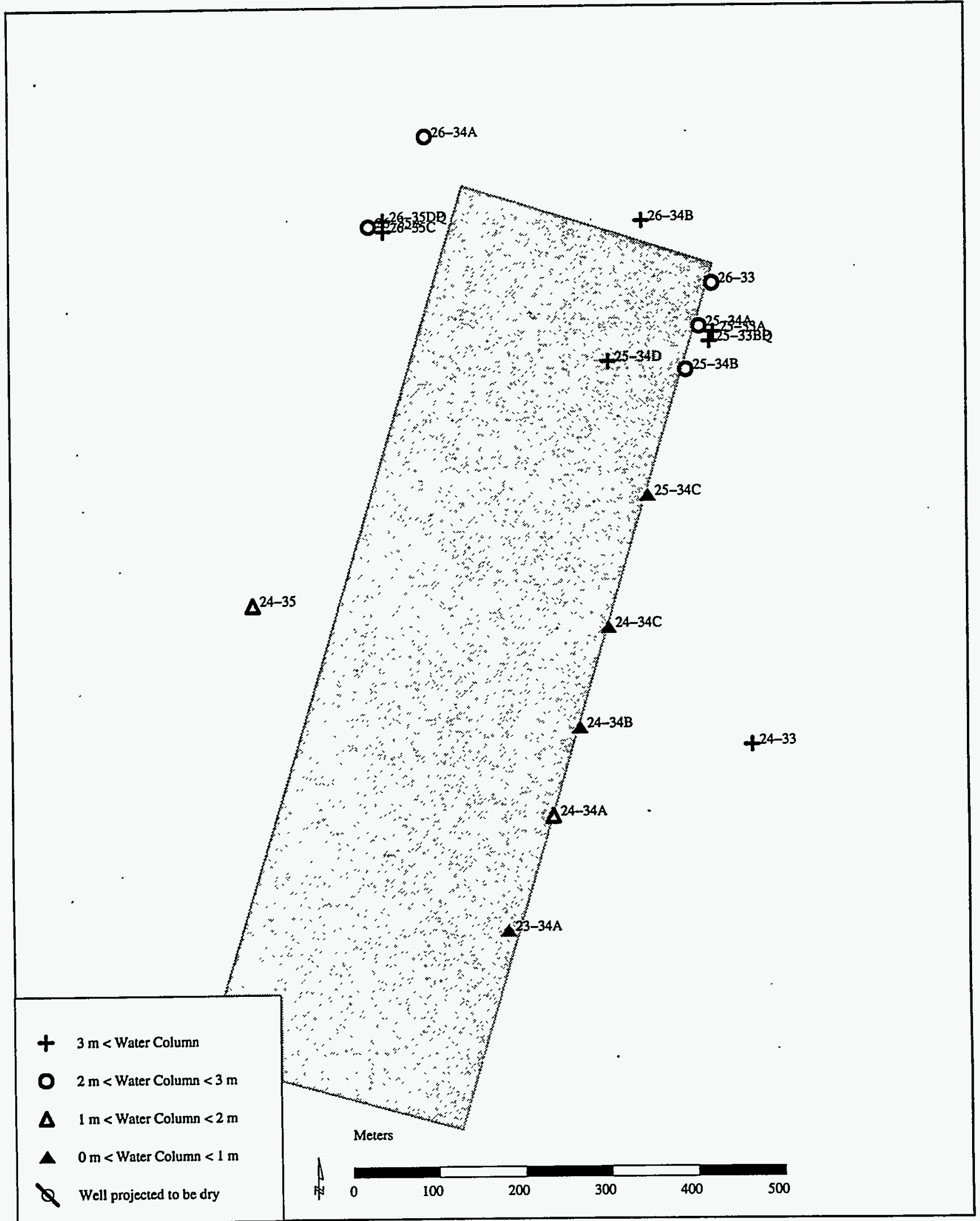

SWL_\&_NRDWL Area selected wells which may be impacted by declining water levels before 1 January 2000 


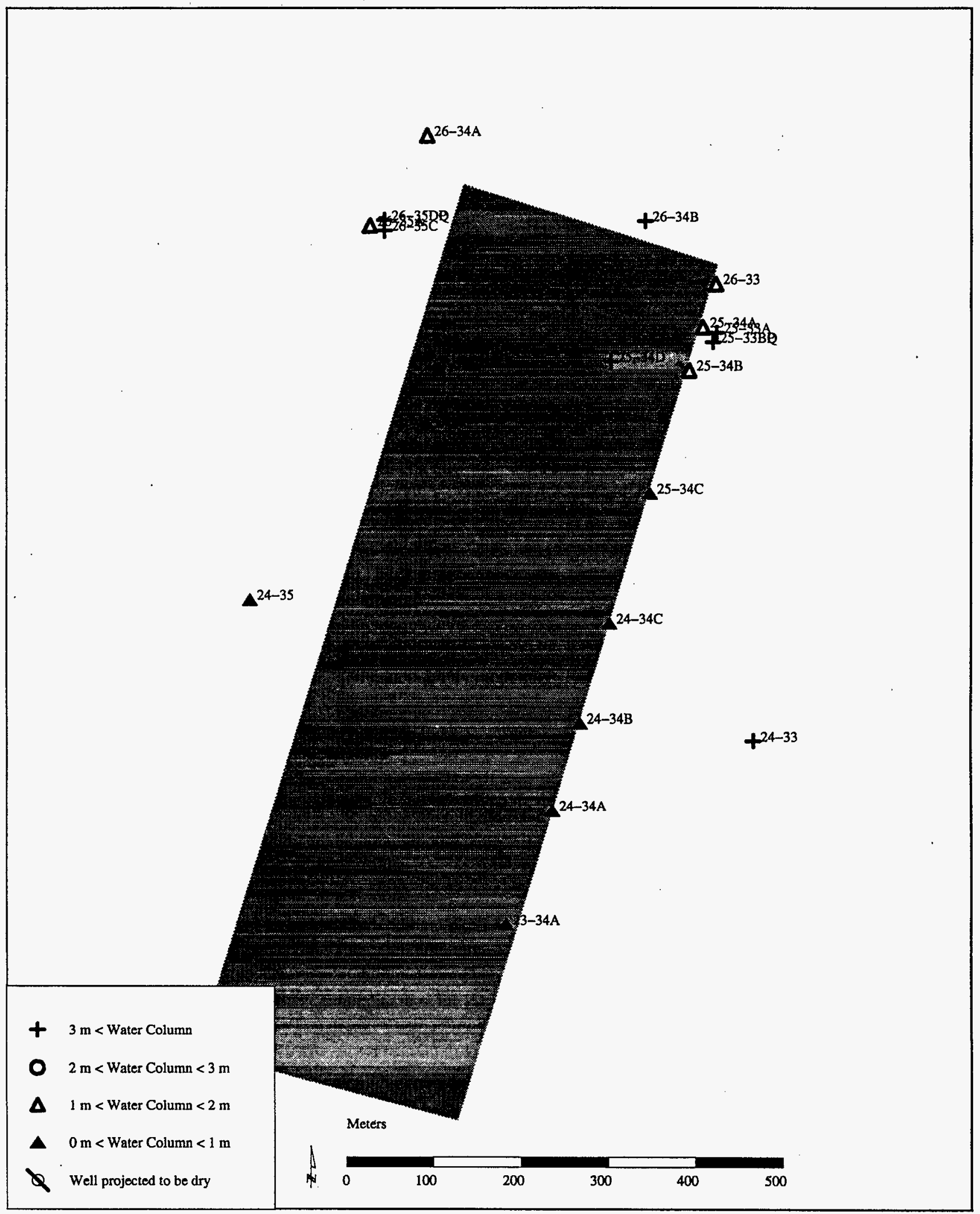

SWL_\&_NRDWL Area selected wells which may be impacted by declining water levels before 1 January 2005

\section{B. 27}




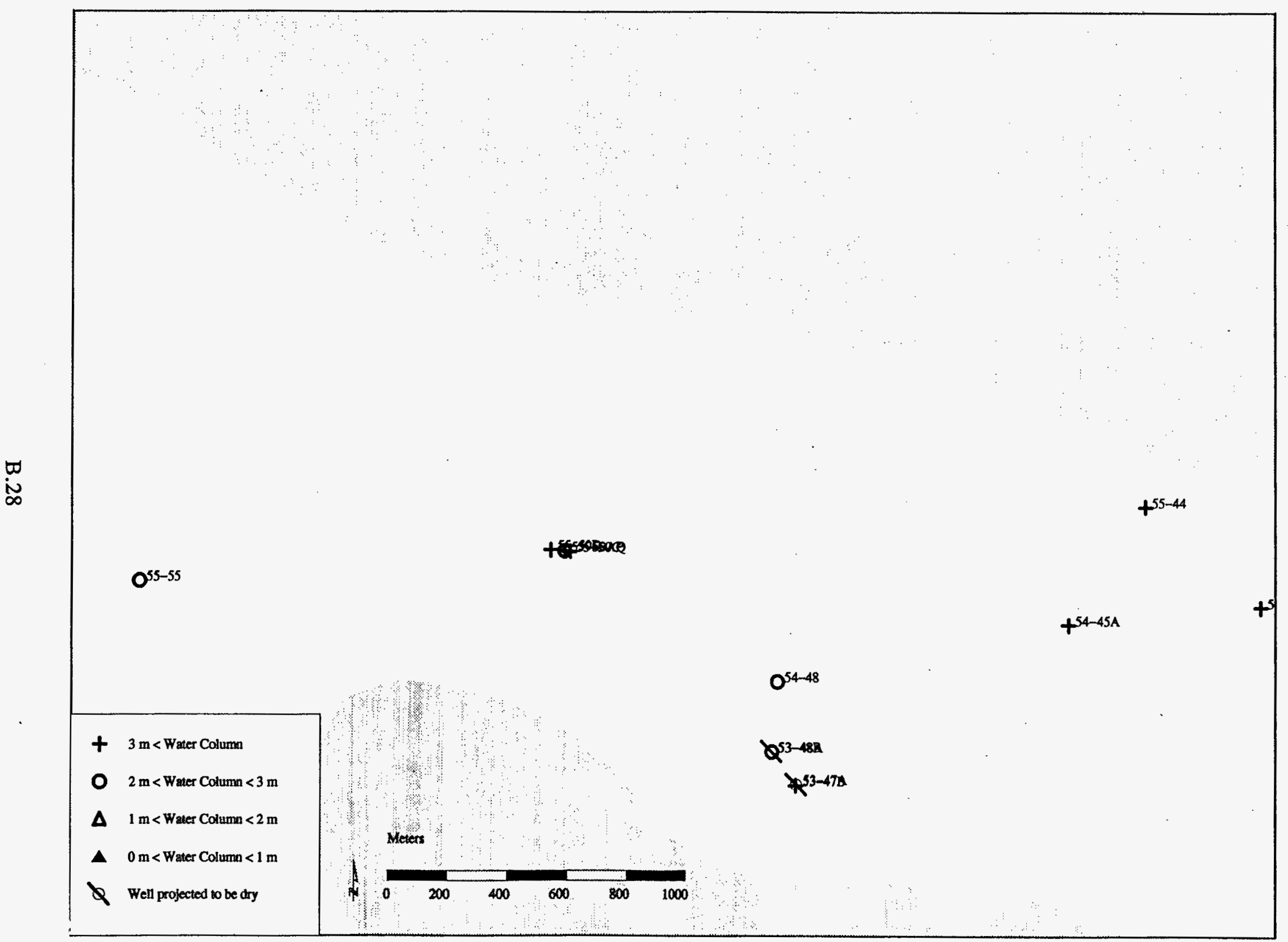

Gable_Mountain Area selected wells which may be impacted by declining water levels before 1 January 1995 


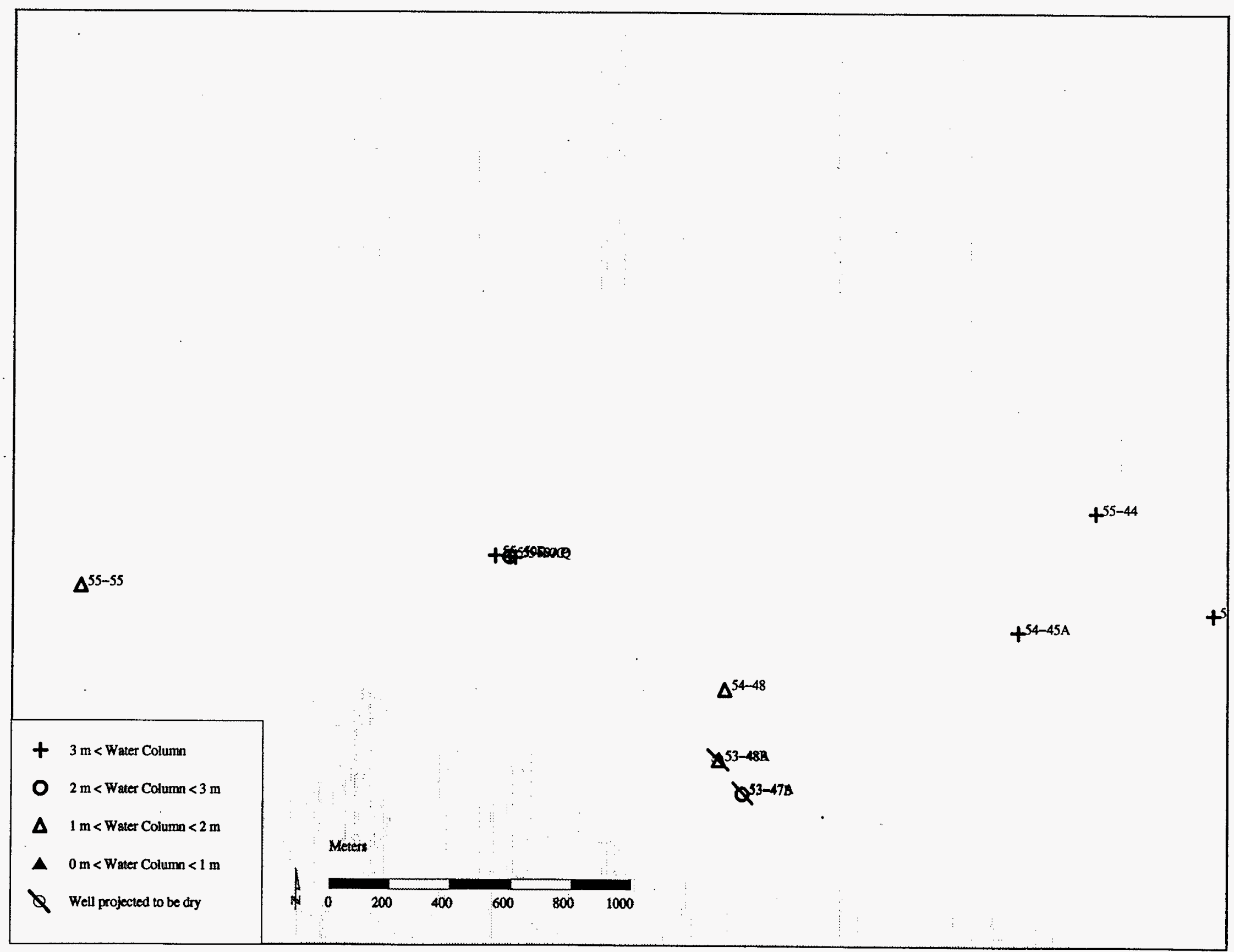

Gable_Mountain Area selected wells which may be impacted by declining water levels before 1 January 2000 


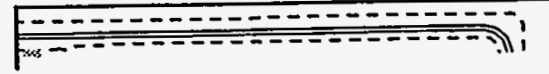

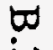

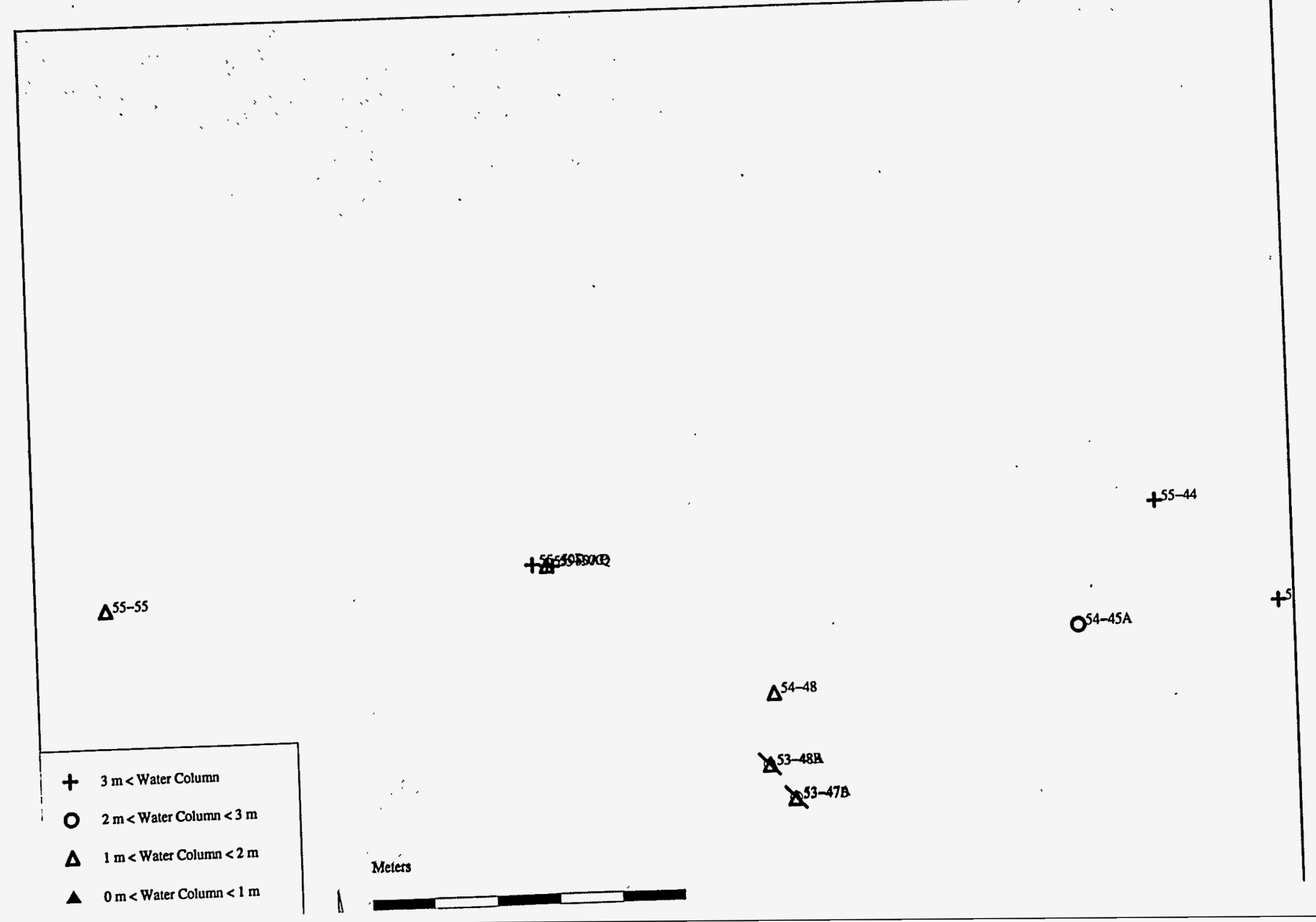




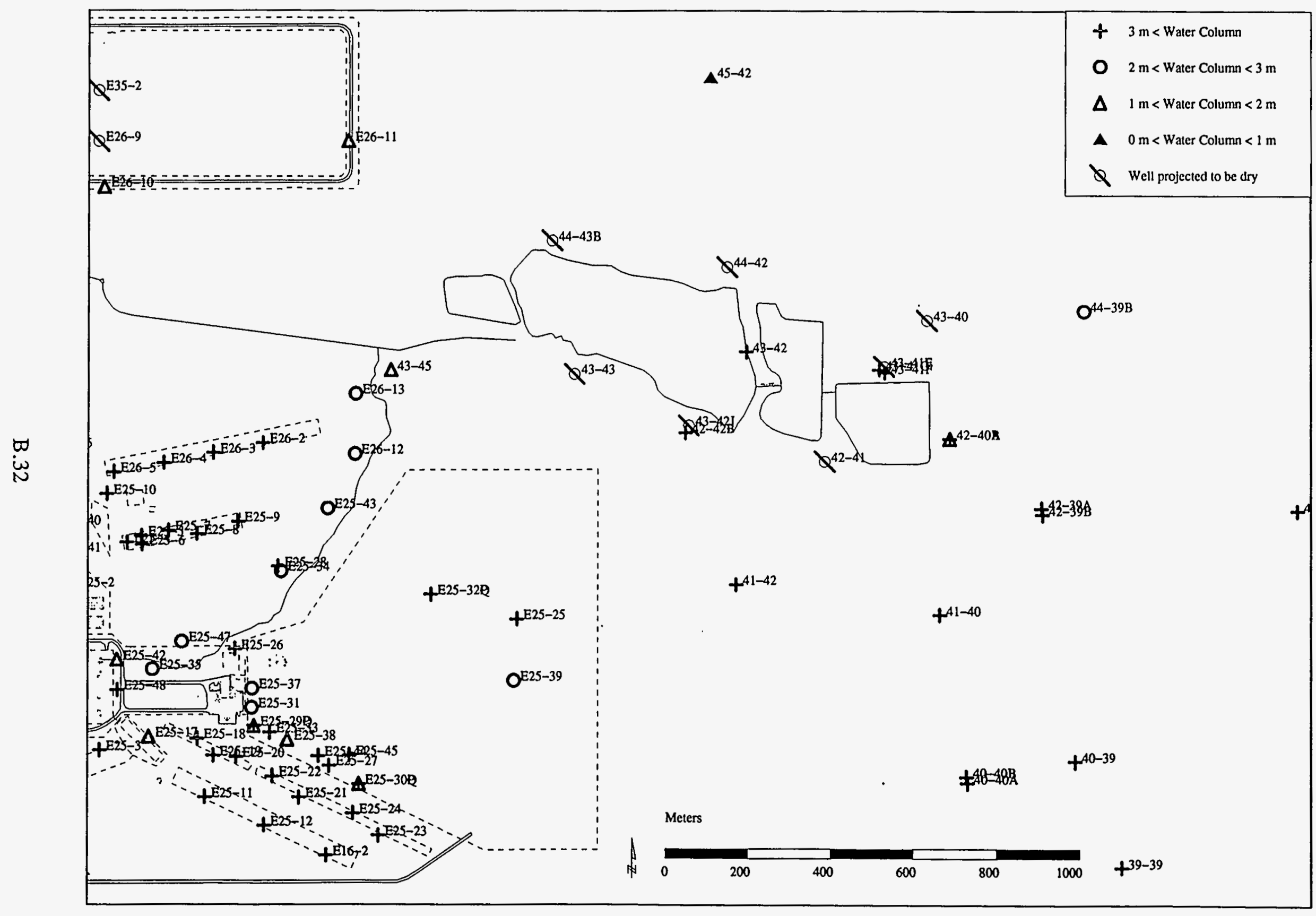

LERF, Grout, A-29 Ditch, and B Pond Area selected wells which may be impacted by declining water levels before 1 January 2000 . 


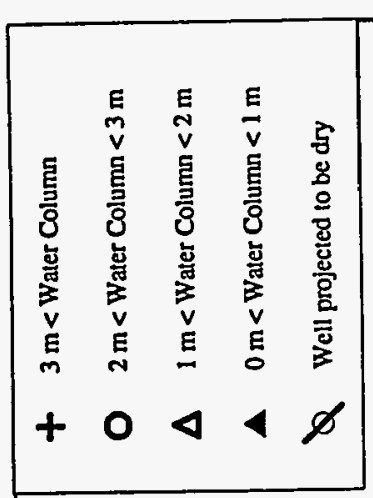

产
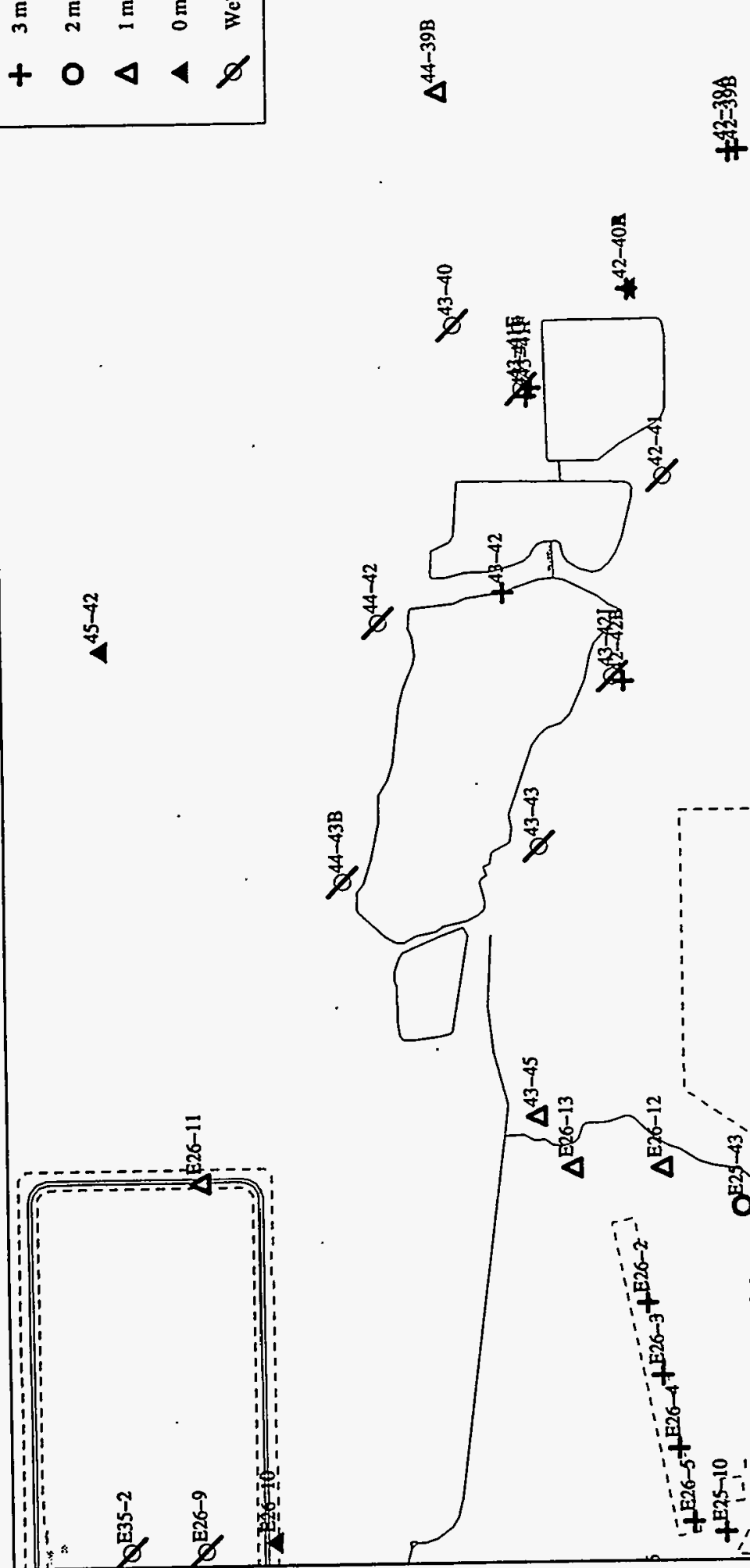

$\frac{8}{7}$

$\frac{7}{7}$

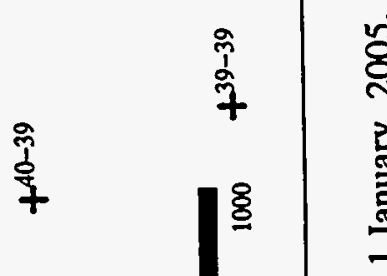

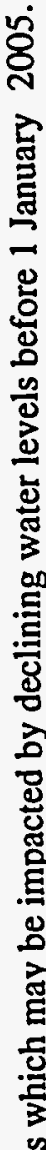

ฟิ

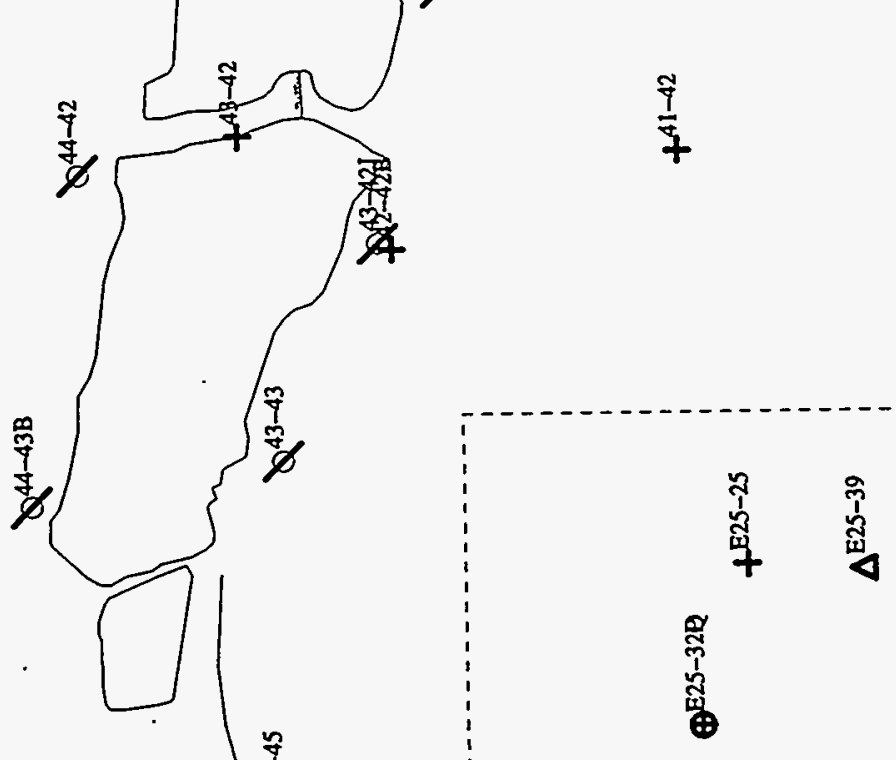

哲

8

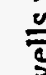

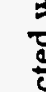

엉

迩

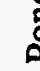

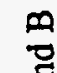

政

돌

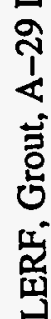


Appendix C

Projected Head Above Bottom of Screen for

Selected Monitoring Wells on the Hanford Site 


\section{Key for Appendix C}

Key for Company:

Orphan $=$ Not claimed by a Contractor Program

PNL $=$ Pacific Northwest Laboratory

WHC $=$ Westinghouse Hanford Company

USACE $=$ U. S. Army Corps of Engineers

PNL-GLDR = Pacific Northwest Laboratory - Golder Associates

WPPSS $=$ Washington Public Power Supply System

Key for Well Owner:

ES\&M $(R O M)=$ RCRA/Operational

Sitewide $=$ Ground-Water Surveillance Project

$\mathrm{ER}=\mathrm{CERCLA}$

TWRS $=$ Tank Farms

PNL-GOLDER = Ground-Water Surveillance Project

WPPSS $=$ Washington Public Power Supply System

FFTF = Fast Flux Test Facility Drinking Water Wells

\section{Key for Pump Type:}

$\mathrm{H}=$ Hydrostar

$\mathrm{S}=$ Submersible

$\mathrm{B}=$ Bailer 


\begin{tabular}{|c|c|c|c|c|c|c|}
\hline & & & Pump & Estimated W & Vater Columr & (m) \\
\hline Wellname & Company & Owner & Type & Dec. 1995 & Dec. 2000 & Dec. 2005 \\
\hline $1199-15-13 \mathrm{C}$ & Orphan & ES\&M (ROM) & & 16.24 & 16.24 & 16.24 \\
\hline $1199-20-13$ & Orphan & ES\&M (ROM) & & 12.6 & 12.6 & 12.6 \\
\hline $1199-22-11 \mathrm{~A}$ & Orphan & ES\&M (ROM) & & 34.55 & 34.53 & 34.53 \\
\hline 1199-34-15A & PNL & Sitewide & & 21.91 & 21.73 & 21.71 \\
\hline 1199-39-16C & PNL & Sitewide & & 11.01 & 10.85 & 10.83 \\
\hline $1199-40-15$ & PNL & Sitewide & & 11.69 & 11.53 & 11.51 \\
\hline $1199-40-16 \mathrm{~B}$ & PNL & Sitewide & & 13.14 & 13 & 12.99 \\
\hline $199-\mathrm{B} 2-13$ & WHC & $\overline{E R}$ & $\mathrm{H}$ & 3.51 & 3.51 & 3.51 \\
\hline 199-B3-1 & WHC & $\overline{E R}$ & $S$ & 4.32 & 4.31 & 4.3 \\
\hline 199-B3-2Q & PNL & Sitewide & & 180.57 & 180.55 & 180.53 \\
\hline 199-B3-46 & WHC & ER & $\mathrm{H}$ & 5.18 & 5.17 & 5.15 \\
\hline 199-B4-1 & WHC & $\overline{E R}$ & $S$ & 7.11 & 7.08 & 7.05 \\
\hline 199-B4-2 & WHC & ER & $\mathrm{B}$ & 5.85 & 5.82 & 5.79 \\
\hline $199-\bar{B} 4-3$ & WHC & $\overline{\mathrm{ER}}$ & $\overline{\mathrm{B}}$ & 5.74 & 5.71 & 5.68 \\
\hline 199-B4-4 & WHC & ER & $S$. & 7.59 & 7.54 & 7.49 \\
\hline 199-B4-5 & PNL & Sitewide & $\mathrm{H}$ & 3.13 & 3.07 & 3.02 \\
\hline 199-B4-6 & WHC & ER & $\mathrm{H}$ & 3.16 & 3.1 & 3.05 \\
\hline 199-B4-7 & PNL & Sitewide & $\mathrm{H}$ & 2.93 & 2.86 & 2.81 \\
\hline 199-B4-8 & WHC & ER & $\overline{\mathrm{H}}$ & 2.09 & 2.03 & 1.99 \\
\hline 199-B4-9 & WHC & ER & $\overline{\mathrm{H}}$ & 1.05 & 1 & 0.95 \\
\hline 199-B5-1 & WHC & ER & $S$ & 11.92 & 11.89 & 11.87 \\
\hline 199-B5-2 & WHC & ER & $\mathrm{H}$ & 2.83 & 2.8 & 2.77 \\
\hline $199-\mathrm{B} 8-6$ & WHC & ER & $\mathrm{H}$ & 2.79 & 2.76 & 2.73 \\
\hline 199-B9-1 & WHC & ER & $S$ & 8.07 & 8 & 7.94 \\
\hline 199-B9-2 & WHC & $\overline{E R}$ & $\overline{\mathrm{H}}$ & 2.53 & 2.46 & 2.4 \\
\hline 199-B9-3 & WHC & ER & & 2.29 & 2.21 & 2.15 \\
\hline 199-D2-5 & WHC & ES\&M (ROM) & $S$ & 3.13 & 3.09 & 3.04 \\
\hline 199-D2-6 & WHC & ES\&M (ROM) & & 4.32 & 4.3 & 4.26 \\
\hline 199-D5-12 & $\overline{\text { WHC }}$ & ES\&M (ROM) & $S$ & 1.36 & 1.33 & 1.29 \\
\hline 199-D5-13 & WHC & ES\&M (ROM) & $\overline{\mathrm{H}}$ & 3.01 & 2.99 & 2.97 \\
\hline 199-D5-14 & WHC & ES\&M (ROM) & & 3.2 & 3.18 & 3.15 \\
\hline 199-D5-15 & WHC & ES\&M (ROM) & $\overline{\mathrm{H}}$ & 3.31 & 3.28 & 3.25 \\
\hline 199-D5-16 & WHC & ES\&M (ROM) & & 2.82 & 2.79 & 2.76 \\
\hline 199-D5-17 & WHC & ES\&M (ROM) & & 3.38 & 3.35 & 3.3 \\
\hline 199-D5-18 & WHC & ES\&M (ROM) & & 3.4 & 3.37 & 3.32 \\
\hline 199-D5-19 & WHC & ES\&M (ROM) & & 4.52 & 4.48 & 4.44 \\
\hline 199-D5-20 & WHC & ES\&M (ROM) & & 3.9 & 3.89 & 3.88 \\
\hline 199-D8-2 & Orphan & $\overline{E S \& M}(\mathrm{ROM})$ & B & 4.26 & 4.25 & 4.24 \\
\hline 199-D8-3 & WHC & ES\&M (ROM) & $S$ & 4.01 & 4 & 3.99 \\
\hline 199-D8-4 & WHC & ES\&M (ROM) & $\overline{\mathrm{H}}$ & 2.78 & 2.77 & 2.76 \\
\hline 199-D8-5 & WHC & ES\&M (ROM) & $\mathrm{H}$ & 4.33 & 4.32 & 4.31 \\
\hline 199-D8-53 & WHC & ES\&M (ROM) & & 3.86 & 3.85 & 3.85 \\
\hline 199-D8-54A & WHC & ES\&M (ROM) & & 4.04 & 4.03 & 4.02 \\
\hline 199-D8-54B & WHC & ES\&M (ROM) & & 24.82 & 24.81 & 24.8 \\
\hline
\end{tabular}




\begin{tabular}{|c|c|c|c|c|c|c|}
\hline$\longdiv { 1 9 9 - D 8 - 5 5 }$ & WHC & ES\&M (ROM) & & 4.14 & 4.14 & 4.13 \\
\hline 199-D8-6 & WHC & $\overline{E S \& M}(\mathrm{ROM})$ & $\mathrm{H}$ & 4.64 & 4.63 & 4.61 \\
\hline 199-F1-2 & WHC & ER & & 2.82 & 2.81 & 2.81 \\
\hline 199-F5-1 & WHC & ES\&M (ROM) & $\mathbf{S}$ & 7.93 & 7.93 & 7.92 \\
\hline 199-F5-2 & Orphan & Orphan & & 17.35 & 17.35 & 17.34 \\
\hline 199-F5-3 & WHC & ES\&M (ROM) & $\bar{B}$ & 7.31 & 7.31 & 7.31 \\
\hline 199-F5-4 & WHC & ES\&M (ROM) & $\mathbf{S}$ & 11.9 & 11.89 & 11.87 \\
\hline $199-\mathrm{F} 5-42$ & WHC & ER & & 4.11 & 4.11 & 4.11 \\
\hline 199-F5-43A & $\overline{W H C}$ & ER & & 4.43 & 4.43 & 4.43 \\
\hline 199-F5-44 & $\overline{\mathrm{WHC}}$ & ER & & 4.66 & 4.66 & 4.66 \\
\hline 199-F5-45 & WHC & $\overline{E R}$ & & 2.57 & 2.57 & 2.55 \\
\hline $199-\mathrm{F} 5-46$ & WHC & $\overline{E R}$ & & 2.89 & 2.89 & 2.88 \\
\hline 199-F5-47 & WHC & ER & & 3.65 & 3.64 & 3.62 \\
\hline 199-F5-48 & WHC & ER & & 2.27 & 2.26 & 2.25 \\
\hline 199-F5-5 & Orphan & Orphan & & 11.55 & 11.55 & 11.54 \\
\hline $199-\mathrm{F} 5-6$ & WHC & ES\&M (ROM) & $\overline{\mathbf{S}}$ & 9.68 & 9.68 & 9.67 \\
\hline 199-F6-1 & WHC & ER & & 4.13 & 4.13 & 4.12 \\
\hline 199-F7-1 & WHC & ER & $\mathbf{S}$ & 12.97 & 12.95 & 12.91 \\
\hline 199-F7-2 & WHC & ER & & 2.62 & 2.61 & 2.58 \\
\hline $199-F 7-3$ & WHC & $\overline{E R}$ & & 3.06 & 3.05 & 3.01 \\
\hline 199-F8-1 & WHC & $\overline{E R}$ & $\mathbf{S}$ & 5.77 & 5.76 & 5.73 \\
\hline 199-F8-2 & $\overline{\text { WHC }}$ & $\overline{E R}$ & $\overline{\mathbf{B}}$ & 4.26 & 4.25 & 4.23 \\
\hline 199-F8-3 & WHC & $\overline{E R}$ & & 0.95 & 0.93 & 0.9 \\
\hline 199-F8-4 & WHC & ER & & 1.52 & 1.52 & 1.5 \\
\hline 199-H3-1 & WHC & ES\&M (ROM) & $\overline{\mathrm{H}}$ & 9.1 & 9.09 & 9.08 \\
\hline 199-H3-2A & WHC & ES\&M (ROM) & $\overline{\mathrm{H}}$ & 3.21 & 3.2 & 3.2 \\
\hline 199-H3-2B & WHC & ES\&M (ROM) & $\overline{\mathrm{H}}$ & 4.24 & 4.24 & 4.24 \\
\hline 199-H3-2C & WHC. & ES\&M (ROM) & $\overline{\mathrm{H}}$ & 21.07 & 21.07 & 21.06 \\
\hline 199-H4-11 & WHC & ES\&M (ROM) & $\overline{\mathrm{H}}$ & 3.9 & 3.9 & 3.9 \\
\hline 199-H4-13 & WHC & ES\&M (ROM) & $\overline{\mathrm{H}}$ & 3.15 & 3.15 & 3.15 \\
\hline 199-H4-14 & WHC & ES\&M (ROM) & $\overline{\mathrm{H}}$ & 2.9 & 2.9 & 2.9 \\
\hline 199-H4-16 & $\overline{\mathrm{WHC}}$ & ES\&M (ROM) & $\overline{\mathrm{H}}$ & 3.09 & 3.09 & 3.08 \\
\hline 199-H4-17 & WHC & ES\&M (ROM) & $\overline{\mathrm{H}}$ & 0.93 & 0.93 & 0.93 \\
\hline 199-H4-18 & WHC & ES\&M (ROM) & $\mathrm{H}$ & 1.52 & 1.52 & 1.52 \\
\hline 199-H4-3 & WHC & ES\&M (ROM) & $\overline{\mathrm{H}}$ & 3.55 & 3.54 & 3.54 \\
\hline 199-H4-45 & $\overline{W H C}$ & ES\&M (ROM) & & 3.89 & 3.89 & 3.88 \\
\hline 199-H4-46 & WHC & ER & & 3.75 & 3.75 & 3.74 \\
\hline 199-H4-47 & WHC & ER & & 3.56 & 3.56 & 3.55 \\
\hline 199-H4-48 & WHC & ER & & 3.32 & 3.32 & 3.32 \\
\hline 199-H4-49 & WHC & ER & & 1.85 & 1.85 & 1.84 \\
\hline $199-\mathrm{H} 4-6$ & WHC & ES\&M (ROM) & $\overline{\mathrm{H}}$ & -2.19 & -2.19 & -2.19 \\
\hline 199-H4-7 & WHC & ES\&M (ROM) & $\overrightarrow{\mathrm{H}}$ & 2.9 & 2.9 & 2.9 \\
\hline 199-H4-8 & WHC & ES\&M (ROM) & $\overline{\mathrm{H}}$ & 1.53 & 1.53 & 1.53 \\
\hline $199-\mathrm{H} 4-9$ & WHC & ES\&M (ROM) & $\overline{\mathrm{H}}$ & 1.48 & 1.48 & 1.48 \\
\hline 199-H5-1A & WHC & ER & & 2.45 & 2.45 & 2.44 \\
\hline 199-H6-1 & WHC & ES\&M (ROM) & & 3.96 & 3.96 & 3.95 \\
\hline
\end{tabular}




\begin{tabular}{|c|c|c|c|c|c|c|}
\hline $199-\mathrm{K}-10$ & Orphan & ES\&M (ROM) & & 27.51 & 27.41 & 27.32 \\
\hline 199-K-11 & WHC & ES\&M (ROM) & $\mathbf{S}$ & 25.69 & 25.59 & 25.5 \\
\hline $199-\mathrm{K}-12$ & Orphan & ES\&M (ROM) & & 19.29 & 19.19 & 19.11 \\
\hline $199-\mathrm{K}-13$ & WHC & ER & & 26.49 & 26.38 & 26.29 \\
\hline 199-K-18 & WHC & ER & & 12.71 & 12.65 & 12.61 \\
\hline 199-K-19 & WHC & ES\&M (ROM) & $S$ & 5.75 & 5.67 & 5.6 \\
\hline $199-\mathrm{K}-20$ & WHC & ES\&M (ROM) & $S$ & 5.41 & 5.36 & 5.32 \\
\hline $199-\mathrm{K}-21$ & WHC & ER & & 5.64 & 5.58 & 5.54 \\
\hline $199-\mathrm{K}-22$ & WHC & ES\&M (ROM) & $\overline{\mathrm{H}}$ & 5.71 & 5.63 & 5.5 \\
\hline $199-\mathrm{K}-23$ & WHC & ER & & 20.45 & 20.32 & 20.23 \\
\hline 199-K-27 & WHC & ES\&M (ROM) & $\mathbf{S}$ & 3.1 & 2.99 & 2.9 \\
\hline 199-K-28 & WHC & ES\&M (ROM) & $S$ & 4.23 & 4.12 & 4.0 \\
\hline $199-\mathrm{K}-29$ & WHC & ES\&M (ROM) & $\mathbf{S}$ & 2.89 & 2.77 & 2.67 \\
\hline 199-K-30 & WHC & ES\&M (ROM) & $\mathbf{S}$ & 3.88 & 3.76 & 3.66 \\
\hline $199-\mathrm{K}-34$ & WHC & ER & & 3.3 & 3.2 & 3.1 \\
\hline 199-K-35 & WHC & ER & & 2.14 & 1.98 & 1.8 \\
\hline $199-\mathrm{K}-36$ & WHC & $\overline{E R}$ & & 2.44 & 2.25 & 2.0 \\
\hline 199-K-37 & WHC & $\overline{E R}$ & & 3 & 2.92 & 2.8 \\
\hline $199-\mathrm{N}-1$ & Orphan & $\overline{\text { ES\&M (ROM) }}$ & & 8.21 & 8.17 & 8.12 \\
\hline $199-N-130$ & Orphan & ES\&M (ROM) & & -4.89 & $\mid-4.93$ & -4.9 \\
\hline 199-N-13P & Orphan & ES\&M (ROM) & & 0.61 & 0.57 & 0.5 \\
\hline $199-\mathrm{N}-14$ & WHC & ES\&M (ROM) & $S$ & 3.69 & 3.67 & 3.6 \\
\hline $199-N-15$ & Orphan & ES\&M (ROM) & $S$ & 2.95 & 2.88 & 2.8 \\
\hline $199-N-16$ & WHC & ES\&M (ROM) & $\bar{S}$ & 3.01 & 2.95 & 2. \\
\hline $199-\mathrm{N}-17$ & WHC & ES\&M (ROM) & $S$ & 2.98 & 2.94 & 2. \\
\hline $199-\mathrm{N}-18$ & WHC & ES\&M (ROM) & B & 2.55 & 2.52 & 2.49 \\
\hline 199-N-19 & WHC & ES\&M (ROM) & $S$ & 3.66 & 3.63 & 3. \\
\hline $199-\mathrm{N}-10$ & Orphan & ES\&M (ROM) & & -2.54 & -2.58 & -2.6 \\
\hline 199-N-1P & Orphan & ES\&M (ROM) & & 9.04 & 9 & 8.9 \\
\hline 199-N-1Q & Orphan & ES\&M (ROM) & & 1.73 & 1.69 & 1.6 \\
\hline $199-\mathrm{N}-2$ & WHC & ES\&M (ROM) & $S$ & 7.02 & 6.98 & 6.93 \\
\hline $199-\mathrm{N}-20$ & WHC & ES\&M (ROM) & $\mathrm{B}$ & 3.08 & 3.04 & 3.01 \\
\hline 199-N-21 & WHC & ES\&M (ROM) & $S$ & 2.75 & 2.72 & 2.6 \\
\hline $199-\mathrm{N}-22$ & PNL & Sitewide & $\bar{B}$ & 3.05 & 3.01 & 2.9 \\
\hline $199-\mathrm{N}-23$ & WHC & ES\&M (ROM) & $\overline{\mathrm{H}}$ & 3 & 2.97 & 2.93 \\
\hline $199-\mathrm{N}-24$ & PNL & Sitewide & $\mathrm{B}$ & 2.95 & 2.92 & 2.89 \\
\hline $199-\mathrm{N}-25$ & WHC & ES\&M (ROM) & $\bar{B}$ & 3.39 & 3.36 & 3.33 \\
\hline $199-\mathrm{N}-26$ & WHC & ES\&M (ROM) & $\overline{\mathrm{H}}$ & 3.53 & 3.49 & 3.4 \\
\hline $199-\mathrm{N}-27$ & WHC & ES\&M (ROM) & $S$ & 2.7 & 2.59 & 2.4 \\
\hline $199-\mathrm{N}-28$ & WHC & ES\&M (ROM) & $S$ & 2.43 & 2.32 & 2.2 \\
\hline $199-\mathrm{N}-29$ & WHC & ES\&M (ROM) & $S$ & 2.38 & 2.28 & 2.19 \\
\hline $199-N-3$ & WHC & ES\&M (ROM) & $S$ & 7.13 & 7.09 & 7.06 \\
\hline $199-N-31$ & WHC & ES\&M (ROM) & $S$ & 2.13 & 2.04 & 1.96 \\
\hline $199-\mathrm{N}-32$ & WHC & ES\&M (ROM) & $S$ & 2.01 & 1.92 & 1.8 \\
\hline $199-\mathrm{N}-33$ & WHC & ES\&M (ROM) & B & 1.09 & 1.01 & 0.9 \\
\hline $199-\mathrm{N}-34$ & WHC & ES\&M (ROM) & $S$ & 2.12 & 2.04 & $1.9^{\prime}$ \\
\hline
\end{tabular}




\begin{tabular}{|c|c|c|c|c|c|c|}
\hline $199-\mathrm{N}-36$ & WHC & ES\&M (ROM) & $S$ & 1.12 & 1.03 & 0.94 \\
\hline $199-N-37$ & PNL & Sitewide & $S$ & 2.24 & 2.15 & 2.07 \\
\hline $199-\mathrm{N}-39$ & PNL & Sitewide & $S$ & 0.32 & 0.24 & 0.16 \\
\hline $199-\mathrm{N}-4$ & $\overline{\mathrm{PNL}}$ & Sitewide & $\mathbf{S}$ & -3.15 & -3.22 & -3.28 \\
\hline $199-N-40$ & WHC & ES\&M (ROM) & & 1.49 & 1.41 & 1.33 \\
\hline 199-N-41 & WHC & ES\&M (ROM) & $S$ & 1.07 & 0.99 & 0.91 \\
\hline $199-\mathrm{N}-42$ & WHC & ES\&M (ROM) & $S$ & 1.87 & 1.78 & 1.69 \\
\hline $199-\mathrm{N}-43$ & Orphan & Orphan & & 2.91 & 2.84 & 2.77 \\
\hline 199-N-46 & $\overline{\text { WHC }}$ & ES\&M (ROM) & & 2.09 & 2.07 & 2.04 \\
\hline $199-N-47$ & $\overline{\mathrm{WHC}}$ & ES\&M (ROM) & $\bar{S}$ & 5.34 & 5.31 & 5.28 \\
\hline $199-N-49$ & $\overline{\mathrm{WHC}}$ & ES\&M (ROM) & $\bar{S}$ & 3.69 & 3.63 & 3.58 \\
\hline $199-N-5$ & PNL & Sitewide & $\overline{\mathrm{B}}$ & 13.02 & 12.97 & 12.92 \\
\hline 199-N-50 & WHC & ES\&M (ROM) & $\bar{S}$ & 6.01 & 5.97 & 5.94 \\
\hline $199-N-51$ & $\overline{\mathrm{WHC}}$ & ES\&M (ROM) & $S$ & 3.17 & 3.17 & 3.16 \\
\hline $199-N-52$ & WHC & ES\&M (ROM) & $S$ & 0.58 & 0.44 & 0.31 \\
\hline 199-N-54 & $\overline{\mathrm{WHC}}$ & ES\&M (ROM) & $\mathrm{H}$ & 1.13 & 1.09 & 1.04 \\
\hline $199-\mathrm{N}-55$ & WHC & ES\&M (ROM) & $\overline{\mathrm{H}}$ & 1.04 & 0.99 & 0.95 \\
\hline $199-\mathrm{N}-56$ & WHC & ES\&M (ROM) & $\overline{\mathrm{H}}$ & 1.22 & 1.17 & 1.13 \\
\hline $199-N-57$ & WHC & ES\&M (ROM) & B & 1.14 & 1.08 & 1.02 \\
\hline $199-N-58$ & $\mathrm{PNL}$ & Sitewide & $\mathrm{H}$ & -1.05 & -1.12 & -1.18 \\
\hline 199-N-59 & WHC & ES\&M (ROM) & $\mathrm{H}$ & 0.35 & 0.28 & 0.21 \\
\hline $199-N-6$ & PNL & Sitewide & $S$ & -2.27 & -2.34 & -2.41 \\
\hline $199-N-60$ & WHC & ES\&M (ROM) & $\overline{\mathrm{H}}$ & -0.79 & -0.86 & -0.93 \\
\hline 199-N-61 & PNL & Sitewide & $\overline{\mathrm{H}}$ & -1.88 & -1.95 & -2.03 \\
\hline $199-N-62$ & WHC & ES\&M (ROM) & & 1.18 & 1.09 & 1 \\
\hline 199-N-63 & WHC & ES\&M (ROM) & & 0.94 & 0.86 & 0.78 \\
\hline $199-N-64$ & WHC & ES\&M (ROM) & B & 0.91 & 0.84 & $0 . \overline{77}$ \\
\hline $199-N-65$ & WHC & ES\&M (ROM) & & 0.92 & 0.86 & 0.79 \\
\hline 199-N-66 & WHC & ES\&M (ROM) & $\mathrm{H}$ & 2.34 & 2.27 & 2.2 \\
\hline 199-N-67 & WHC & ES\&M (ROM) & $\mathrm{B}$ & 3.6 & 3.55 & 3.5 \\
\hline $199-N-69$ & WHC & ES\&M (ROM) & $\mathrm{H}$ & 8.88 & 8.84 & 8.79 \\
\hline 199-N-70 & $\mathrm{WHC}$ & ES\&M (ROM) & $\mathrm{H}$ & 10.13 & 10.05 & 9.97 \\
\hline $199-N-71$ & WHC & ES\&M (ROM) & $\overline{\mathrm{H}}$ & 3.55 & 3.41 & 3.28 \\
\hline 199-N-72 & WHC & ES\&M (ROM) & $\mathrm{H}$ & 3.74 & 3.67 & 3.6 \\
\hline 199-N-73 & WHC & ES\&M (ROM) & $\overline{\mathrm{H}}$ & 3.75 & 3.67 & 3.6 \\
\hline $199-N-74$ & WHC & ES\&M (ROM) & $\overline{\mathrm{H}}$ & 3.57 & 3.43 & 3.29 \\
\hline $199-N-75$ & WHC & ES\&M (ROM) & $\overline{\mathrm{H}}$ & 4.72 & 4.68 & 4.65 \\
\hline $199-N-76$ & WHC & ES\&M (ROM) & $\overline{\mathrm{H}}$ & 5.08 & 5.05 & 5.02 \\
\hline 199-N-77 & WHC & ES\&M (ROM) & $\mathrm{H}$ & 7.37 & 7.3 & 7.24 \\
\hline 199-N-80 & WHC & ER & & 15.41 & 15.38 & 15.34 \\
\hline 199-N-81 & WHC & ES\&M (ROM) & $\mathrm{H}$ & 5.79 & 5.71 & 5.63 \\
\hline 199-N-8P & $\overline{\mathrm{WHC}}$ & ES\&M (ROM) & $\mathrm{B}$ & 25.13 & 25.1 & 25.07 \\
\hline 199-N-8Q & WHC & ES\&M (ROM) & & 13.09 & 13.06 & 13.03 \\
\hline 199-N-8R & WHC & ES\&M (ROM) & B & 9.28 & 9.26 & 9.23 \\
\hline $199-N-8 S$ & WHC & ES\&M (ROM) & $\mathrm{B}$ & 6.4 & 6.37 & 6.34 \\
\hline $199-N-8 \mathrm{~T}$ & WHC & ES\&M (ROM) & $S$ & 3.95 & 3.92 & 3.89 \\
\hline
\end{tabular}




\begin{tabular}{|c|c|c|c|c|c|c|}
\hline $199-N-8 U$ & WHC & ES\&M (ROM) & & 0.9 & 0.88 & 0.85 \\
\hline 299-E13-1 & WHC & ES\&M (ROM) & & 7.64 & 6.82 & 6.21 \\
\hline 299-E13-10 & WHC & ES\&M (ROM) & & 1.9 & 1.08 & 0.47 \\
\hline 299-E13-11 & $\overline{W H C}$ & ES\&M (ROM) & & 9.77 & 8.95 & 8.34 \\
\hline 299-E13-12 & WHC & ES\&M (ROM) & & 9.68 & 8.86 & 8.25 \\
\hline 299-E13-13 & Orphan & ES\&M (ROM) & & 10.05 & 9.23 & 8.62 \\
\hline 299-E13-14 & WHC & ES\&M (ROM) & $\mathbf{S}$ & 3.34 & 2.52 & 1.91 \\
\hline 299-E13-15 & WHC & ES\&M (ROM) & & 10.25 & 9.42 & 8.81 \\
\hline 299-E13-16 & WHC & ES\&M (ROM) & & 7.18 & 6.36 & 5.75 \\
\hline 299-E13-17 & WHC & ES\&M (ROM) & & 8.04 & 7.21 & 6.6 \\
\hline 299-E13-1-8 & WHC & ES\&M (ROM) & & 25.59 & 24.76 & 24.15 \\
\hline 299-E13-19 & WHC & ES\&M (ROM) & B & 10.54 & 9.72 & 9.11 \\
\hline 299-E13-2 & WHC & ES\&M (ROM) & & 7.46 & 6.64 & 6.03 \\
\hline 299-E13-20 & WHC & ES\&M (ROM) & & 74.39 & 73.57 & 72.96 \\
\hline 299-E13-3 & WHC & ES\&M (ROM) & & 7.16 & 6.34 & 5.73 \\
\hline 299-E13-4 & WHC & ES\&M (ROM) & & 8.12 & 7.29 & 6.69 \\
\hline 299-E13-5 & PNL & Sitewide & $S$ & 7.59 & 6.77 & 6.16 \\
\hline 299-E13-6 & WHC & ES\&M (ROM) & & 6.88 & 6.06 & 5.45 \\
\hline 299-E13-7 & WHC & ES\&M (ROM) & & 7.64 & 6.82 & 6.21 \\
\hline 299-E13-8 & WHC & ES\&M (ROM) & $\mathrm{B}$ & 7.42 & 6.59 & 5.98 \\
\hline 299-E13-9 & WHC & ES\&M (ROM) & & 6.4 & 5.58 & 4.97 \\
\hline 299-E16-2 & WHC & ES\&M (ROM) & $\mathrm{B}$ & 17.58 & 16.76 & 16.15 \\
\hline 299-E17-1 & WHC & ES\&M (ROM) & $S$ & 5.07 & 4.25 & 3.65 \\
\hline 299-E17-10 & WHC & ES\&M (ROM) & $S$ & 2.46 & 1.64 & 1.03 \\
\hline 299-E17-12 & WHC & ES\&M (ROM) & $S$ & 5.82 & 5 & 4.4 \\
\hline 299-E17-13 & WHC & ES\&M (ROM) & $S$ & 6.26 & 5.45 & 4.84 \\
\hline 299-E17-14 & WHC & ES\&M (ROM) & $\mathrm{H}$ & 3.69 & 2.87 & 2.26 \\
\hline 299-E17-15 & WHC & ES\&M (ROM) & $\mathrm{H}$ & 3.23 & 2.42 & 1.81 \\
\hline 299-E17-16 & WHC & ES\&M (ROM) & $\mathrm{H}$ & 3.66 & 2.84 & 2.24 \\
\hline 299-E17-17 & WHC & ES\&M (ROM) & $\mathrm{H}$ & 3.94 & 3.12 & 2.51 \\
\hline 299-E17-18 & WHC & ES\&M (ROM) & $\mathrm{H}$ & 3.49 & 2.67 & 2.06 \\
\hline 299-E17-19 & WHC & ES\&M (ROM) & $\mathrm{H}$ & 2.42 & 1.6 & 0.99 \\
\hline 299-E17-2 & PNL & Sitewide & $\mathrm{B}$ & 9.06 & 8.24 & 7.64 \\
\hline 299-E17-20 & WHC & ES\&M (ROM) & $\mathrm{H}$ & 2.21 & 1.39 & 0.78 \\
\hline 299-E17-3 & WHC & ES\&M (ROM) & $\bar{B}$ & 26.62 & 25.8 & 25.19 \\
\hline 299-E17-4 & WHC & ES\&M (ROM) & $\bar{B}$ & 25.53 & 24.71 & 24.1 \\
\hline 299-E17-5 & WHC & ES\&M (ROM) & $S$ & 5.82 & 5 & 4.4 \\
\hline 299-E17-6 & WHC & ES\&M (ROM) & $\underline{S}$ & 43.49 & 42.67 & 42.07 \\
\hline 299-E17-7 & WHC & ES\&M (ROM) & $B$ & 20.91 & 20.09 & 19.49 \\
\hline 299-E17-8 & WHC & ES\&M (ROM) & $S$ & 8.06 & 7.24 & 6.63 \\
\hline 299-E17-9 & WHC & ES\&M (ROM) & $S$ & 1.57 & 0.75 & 0.15 \\
\hline 299-E18-1 & WHC & ES\&M (ROM) & $\overline{\mathrm{H}}$ & 3.59 & 2.77 & 2.16 \\
\hline 299-E18-2 & WHC & ES\&M (ROM) & $\mathrm{H}$ & 3.48 & 2.66 & 2.05 \\
\hline 299-E18-3 & WHC & ES\&M (ROM) & $\mathrm{H}$ & 3.3 & 2.48 & 1.87 \\
\hline 299-E18-4 & WHC & ES\&M (ROM) & $\mathrm{H}$ & 2.99 & 2.16 & 1.55 \\
\hline 299-E19-1 & $\overline{\mathrm{WHC}}$ & ES\&M (ROM) & $\bar{B}$ & 11.5 & 10.67 & 10.06 \\
\hline
\end{tabular}




\begin{tabular}{|c|c|c|c|c|c|c|}
\hline 299-E23-1 & WHC & ES\&M (ROM) & $\bar{B}$ & 9.99 & 9.17 & 8.56 \\
\hline 299-E23-2 & WHC & ES\&M (ROM) & $\bar{B}$ & 35.74 & 34.92 & 34.31 \\
\hline 299-E23-20 & WHC & ES\&M (ROM) & & 6.6 & 5.77 & 5.17 \\
\hline 299-E23-2P & WHC & ES\&M (ROM) & & 41.95 & 41.13 & 40.52 \\
\hline 299-E23-2Q & WHC & ES\&M (ROM) & & 24.88 & 24.06 & 23.46 \\
\hline 299-E24-1 & PNL & Sitewide & $\bar{B}$ & 8.41 & 7.59 & 6.99 \\
\hline 299-E24-11 & $\overline{\text { PNL }}$ & Sitewide & $\overline{\mathrm{B}}$ & 7.14 & 6.32 & 5.72 \\
\hline 299-E24-12 & PNL & Sitewide & $S$ & 0.16 & -0.66 & -1.27 \\
\hline 299-E24-13 & $\overline{\mathrm{WHC}}$ & ES\&M (ROM) & $\overline{\mathrm{B}}$ & 6 & 5.18 & 4.58 \\
\hline 299-E24-14 & WHC & ES\&M (ROM) & & 15.09 & 14.27 & 13.66 \\
\hline 299-E24-16 & WHC & ES\&M (ROM) & $\mathrm{H}$ & 2.72 & 1.9 & 1.29 \\
\hline 299-E24-17 & WHC & ES\&M (ROM) & $\overline{\mathrm{H}}$ & 3.98 & 3.16 & 2.56 \\
\hline 299-E24-18 & WHC & ES\&M (ROM) & $\mathrm{H}$ & 3.37 & 2.55 & 1.94 \\
\hline 299-E24-19 & WHC & ES\&M (ROM) & $\mathrm{H}$ & 3 & 2.18 & 1.58 \\
\hline 299-E24-2 & WHC & ES\&M (ROM) & $S$ & 10.16 & 9.34 & 8.74 \\
\hline 299-E24-20 & WHC & ES\&M (ROM) & $\bar{H}$ & 4.02 & 3.2 & 2.59 \\
\hline 299-E24-3 & WHC & ES\&M (ROM) & & 10.82 & 10 & 9.39 \\
\hline 299-E24-4 & PNL & Sitewide & $\bar{S}$ & 1.26 & 0.44 & -0.17 \\
\hline 299-E24-5 & $\overline{\mathrm{WHC}}$ & ES\&M (ROM) & $\bar{B}$ & 10.13 & 9.3 & 8.7 \\
\hline 299-E24-7 & WHC & ES\&M (ROM) & $S$ & 11.23 & 10.41 & 9.8 \\
\hline 299-E24-8 & WHC & ES\&M (ROM) & $\bar{S}$ & 26.23 & 25.4 & 24.8 \\
\hline 299-E25-1 & WHC & ES\&M (ROM) & $\bar{B}$ & 6.78 & 5.96 & 5.36 \\
\hline 299-E25-10 & WHC & ES\&M (ROM) & $\mathbf{S}$ & 11.58 & 10.75 & 10.15 \\
\hline 299-E25-11 & WHC & ES\&M (ROM) & $\overline{\mathrm{H}}$ & 17.22 & 16.4 & 15.8 \\
\hline 299-E25-12 & WHC & ES\&M (ROM) & $\bar{B}$ & 18.23 & 17.41 & 16.81 \\
\hline 299-E25-13 & WHC & ES\&M (ROM) & $\mathrm{B}$ & 7.74 & 6.92 & 6.31 \\
\hline 299-E25-15 & WHC & ES\&M (ROM) & & 15.57 & 14.75 & 14.15 \\
\hline 299-E25-16 & WHC & ES\&M (ROM) & & 15.14 & 14.31 & 13.71 \\
\hline 299-E25-17 & PNL & Sitewide & $\bar{B}$ & 2.38 & 1.56 & 0.95 \\
\hline 299-E25-18 & WHC & ES\&M (ROM) & $S$ & 5.41 & 4.59 & 3.98 \\
\hline 299-E25-19 & WHC & ES\&M (ROM) & $S$ & 6.28 & 5.45 & 4.85 \\
\hline 299-E25-2 & WHC & ES\&M (ROM) & $\bar{B}$ & 13.22 & 12.4 & 11.79 \\
\hline 299-E25-20 & WHC & ES\&M (ROM) & $S$ & 5.94 & 5.12 & 4.51 \\
\hline 299-E25-21 & $\overline{W H C}$ & ES\&M (ROM) & S & 5.64 & 4.82 & 4.22 \\
\hline 299-E25-22 & $\overline{\mathrm{WHC}}$ & ES\&M (ROM) & $S$ & 7.24 & 6.42 & 5.82 \\
\hline 299-E25-23 & PNL & Sitewide & S & 5.37 & 4.55 & 3.95 \\
\hline 299-E25-24 & WHC & ES\&M (ROM) & $S$ & 4.03 & 3.21 & 2.6 \\
\hline $299-\mathrm{E} 25-25$ & $\overline{\mathrm{WHC}}$ & ES\&M (ROM) & $\mathrm{H}$ & 6.84 & 6.01 & 5.41 \\
\hline 299-E25-26 & $\overline{\mathrm{WHC}}$ & ES\&M (ROM) & $S$ & 7.4 & 6.58 & 5.98 \\
\hline 299-E25-27 & $\overline{W H C}$ & ES\&M (ROM) & $\bar{B}$ & 6.31 & 5.49 & 4.88 \\
\hline 299-E25-28 & $\overline{\mathrm{WHC}}$ & ES\&M (ROM) & s & 24.5 & 23.68 & 23.07 \\
\hline 299-E25-29P & WHC & ES\&M (ROM) & $\mathrm{H}$ & 1.9 & 1.08 & 0.47 \\
\hline 299-E25-29Q & WHC & ES\&M (ROM) & & 18.29 & 17.4 .6 & 16.86 \\
\hline 299-E25-3 & $\overline{\mathrm{PNL}}$ & Sitewide & B & 6.64 & 5.82 & 5.21 \\
\hline 299-E25-30P & $\overline{\mathrm{WHC}}$ & ES\&M (ROM) & $\overline{\mathrm{H}}$ & 16.63 & 15.81 & 15.21 \\
\hline 299-E25-30Q & WHC & ES\&M (ROM) & & 2.55 & 1.73 & 1.12 \\
\hline
\end{tabular}




\begin{tabular}{|c|c|c|c|c|c|c|}
\hline 299-E25-31 & WHC & ES\&M (ROM) & $\mathrm{H}$ & 2.82 & 2 & 1.4 \\
\hline 299-E25-32P & WHC & ES\&M (ROM) & $\bar{H}$ & 19.4 & 18.58 & 17.97 \\
\hline 299-E25-32Q & WHC & ES\&M (ROM) & & 4.32 & 3.49 & 2.89 \\
\hline 299-E25-33 & WHC & ES\&M (ROM) & $\mathrm{H}$ & 10.6 & 9.78 & 9.17 \\
\hline 299-E25-34 & WHC & ES\&M (ROM) & $\overline{\mathrm{H}}$ & 3.52 & 2.7 & 2.09 \\
\hline 299-E25-35 & WHC & ES\&M (ROM) & $\overline{\mathrm{H}}$ & 2.87 & 2.05 & 1.44 \\
\hline 299-E25-36 & WHC & ES\&M (ROM) & $\mathrm{H}$ & 3.85 & 3.03 & 2.42 \\
\hline 299-E25-37 & WHC & ES\&M (ROM) & $\overline{\mathrm{H}}$ & 2.9 & 2.08 & 1.47 \\
\hline 299-E25-38 & WHC & ES\&M (ROM) & $\mathrm{H}$ & 2.71 & 1.89 & 1.29 \\
\hline 299-E25-39 & WHC & ES\&M (ROM) & $\mathrm{H}$ & 2.87 & 2.04 & 1.44 \\
\hline 299-E25-4 & WHC & ES\&M (ROM) & $\overline{\mathrm{B}}$ & 7.45 & 6.62 & 6.02 \\
\hline 299-E25-40 & WHC & ES\&M (ROM) & $\mathrm{H}$ & 3.09 & 2.26 & 1.66 \\
\hline 299-E25-41 & WHC & ES\&M (ROM) & $\mathrm{H}$ & 2.41 & 1.59 & 0.98 \\
\hline 299-E25-42 & WHC & ES\&M (ROM) & $\overline{\mathrm{H}}$ & 2.57 & 1.74 & 1.14 \\
\hline 299-E25-43 & WHC & ES\&M (ROM) & $\mathrm{H}$ & 3.77 & 2.94 & 2.33 \\
\hline 299-E25-44 & WHC & $\overline{E S \& M}(\mathrm{ROM})$ & $\vec{H}$ & 4.08 & 3.26 & 2.66 \\
\hline 299-E25-45 & $\overline{\mathrm{WHC}}$ & ES\&M (ROM) & $\overline{\mathrm{H}}$ & 4.25 & 3.43 & 2.82 \\
\hline 299-E25-46 & WHC & ES\&M (ROM) & $\overline{\mathrm{H}}$ & 4.36 & 3.54 & 2.93 \\
\hline 299-E25-47 & WHC & ES\&M (ROM) & $\mathrm{H}$ & 3.73 & 2.91 & 2.3 \\
\hline 299-E25-48 & WHC & $\overline{\mathrm{ES} \& \mathrm{M}(\mathrm{ROM})}$ & $\overline{\mathrm{H}}$ & 4.6 & 3.78 & 3.17 \\
\hline 299-E25-5 & WHC & $\overline{\mathrm{ES} \& \mathrm{M}(\mathrm{ROM})}$ & & 11.01 & 10.18 & 9.58 \\
\hline 299-E25-6 & WHC & ES\&M (ROM) & B & 9.91 & 9.09 & 8.48 \\
\hline 299-E25-7 & WHC & ES\&M (ROM) & & 10.88 & 10.05 & 9.45 \\
\hline 299-E25-8 & WHC & ES\&M (ROM) & & $8: 69$ & 7.87 & 7.26 \\
\hline 299-E25-9 & WHC & ES\&M (ROM) & $B$ & 10.97 & 10.14 & 9.53 \\
\hline 299-E26-1 & $\overline{\mathrm{WHC}}$ & ES\&M (ROM) & $\bar{B}$ & 3.84 & 3.01 & 2.41 \\
\hline 299-E26-10 & WHC & ES\&M (ROM) & $\overline{\mathrm{H}}$ & 2.28 & 1.45 & 0.85 \\
\hline 299-E26-11 & WHC & ES\&M (ROM) & $\overline{\mathrm{H}}$ & 2.75 & 1.91 & 1.3 \\
\hline 299-E26-12 & WHC & ES\&M (ROM) & H & 3.27 & 2.44 & 1.83 \\
\hline 299-E26-13 & WHC & ES\&M (ROM) & $\mathrm{H}$ & 3.1 & 2.27 & 1.66 \\
\hline 299-E26-2 & WHC & ES\&M (ROM) & $\bar{B}$ & 9.92 & 9.09 & 8.49 \\
\hline 299-E26-3 & WHC & ES\&M (ROM) & $\bar{B}$ & 10.26 & 9.43 & 8.83 \\
\hline 299-E26-4 & WHC & ES\&M (ROM) & B & 11 & 10.17 & 9.57 \\
\hline 299-E26-5 & WHC & ES\&M (ROM) & $B$ & 12.73 & 11.9 & 11.3 \\
\hline 299-E26-6 & WHC & ES\&M (ROM) & $\bar{B}$ & 14.65 & 13.82 & 13.22 \\
\hline 299-E26-9 & WHC & ES\&M (ROM) & $\mathrm{H}$ & 0.27 & -0.56 & -1.16 \\
\hline 299-E27-1 & WHC & ES\&M (ROM) & $\bar{B}$ & 15.64 & 14.81 & 14.21 \\
\hline 299-E27-10 & WHC & ES\&M (ROM) & $\mathrm{H}$ & 3.28 & 2.45 & 1.85 \\
\hline 299-E27-11 & WHC & ES\&M (ROM) & $\bar{H}$ & 3.33 & 2.5 & 1.9 \\
\hline 299-E27-12 & WHC & ES\&M (ROM) & $\mathrm{H}$ & 2.88 & 2.05 & 1.45 \\
\hline 299-E27-13 & WHC & ES\&M (ROM) & $\mathrm{H}$ & 2.6 & 1.77 & 1.17 \\
\hline 299-E27-14 & WHC & ES\&M (ROM) & $\mathrm{H}$ & 3.45 & 2.63 & 2.02 \\
\hline 299-E27-15 & WHC & ES\&M (ROM) & $\mathrm{H}$ & 2.81 & 1.98 & 1.37 \\
\hline 299-E27-16 & WHC & ES\&M (ROM) & $\mathrm{H}$ & 3.17 & 2.34 & 1.74 \\
\hline 299-E27-17 & WHC & ES\&M (ROM) & $\overline{\mathrm{H}}$ & 3.74 & 2.91 & 2.31 \\
\hline 299-E27-18 & WHC & ES\&M (ROM) & $\mathrm{H}$ & 4.41 & 3.59 & 2.99 \\
\hline
\end{tabular}




\begin{tabular}{|c|c|c|c|c|c|c|}
\hline 299-E27-19 & WHC & ES\&M (ROM) & $\overline{\mathrm{H}}$ & 4.37 & 3.55 & 2.95 \\
\hline 299-E27-3 & $\overline{\mathrm{WHC}}$ & ES\&M (ROM) & & 20.59 & 19.77 & 19.16 \\
\hline 299-E27-30 & WHC & ES\&M (ROM) & . & 25.61 & 24.78 & 24.18 \\
\hline 299-E27-3P & WHC & ES\&M (ROM) & & 24.08 & 23.26 & 22.65 \\
\hline 299-E27-5 & WHC & ES\&M (ROM) & $\mathbf{S}$ & 15.5 & 14.67 & 14.07 \\
\hline 299-E27-7 & WHC & ES\&M (ROM) & $\bar{S}$ & 14.99 & 14.16 & 13.56 \\
\hline 299-E27-8 & WHC & ES\&M (ROM) & $\mathrm{H}$ & 3.2 & 2.38 & 1.77 \\
\hline 299-E27-9 & WHC & ES\&M (ROM) & $\overline{\mathrm{H}}$ & 3.87 & 3.05 & 2.44 \\
\hline 299-E28-1 & PNL & Sitewide & $\mathbf{S}$ & 12.08 & 11.26 & 10.66 \\
\hline 299-E28-10 & WHC & ES\&M (ROM) & $\bar{B}$ & 10.42 & 9.59 & 8.99 \\
\hline 299-E28-16 & PNL & Sitewide & $S$ & 6.94 & 6.12 & 5.51 \\
\hline 299-E28-17 & WHC & ES\&M (ROM) & B & 8.94 & 8.12 & 7.51 \\
\hline 299-E28-18 & WHC & ES\&M (ROM) & $\mathbf{S}$ & 10.76 & 9.93 & 9.33 \\
\hline 299-E28-19 & $\overline{\mathrm{PNL}}$ & Sitewide & $\bar{S}$ & 9.26 & 8.44 & 7.84 \\
\hline 299-E28-2 & WHC & ES\&M (ROM) & $\bar{B}$ & 12.17 & 11.35 & 10.75 \\
\hline 299-E28-20 & $\overline{\mathrm{WHC}}$ & ES\&M (ROM) & & 11.46 & 10.63 & 10.03 \\
\hline 299-E28-21 & WHC & ES\&M (ROM) & $S$ & 11.92 & 11.1 & 10.5 \\
\hline 299-E28-23 & WHC & ES\&M (ROM) & $S$ & 13.67 & 12.85 & 12.24 \\
\hline 299-E28-24 & PNL & Sitewide & $\mathrm{B}$ & 13.37 & 12.54 & 11.94 \\
\hline 299-E28-25 & PNL & Sitewide & $\mathrm{B}$ & 13.98 & 13.15 & 12.55 \\
\hline 299-E28-26 & WHC & ES\&M (ROM) & $\mathrm{H}$ & 4.39 & 3.56 & 2.96 \\
\hline 299-E28-27 & WHC & ES\&M (ROM) & $\mathrm{H}$ & 3.77 & 2.95 & 2.34 \\
\hline 299-E28-28 & WHC & ES\&M (ROM) & $\mathrm{H}$ & 3.44 & 2.62 & 2.02 \\
\hline 299-E28-3 & WHC & ES\&M (ROM) & & $10: 36$ & 9.54 & 8.94 \\
\hline 299-E28-4 & $\overline{\mathrm{WHC}}$ & ES\&M (ROM) & $\mathrm{B}$ & 9.85 & 9.02 & 8.42 \\
\hline 299-E28-5 & PNL & Sitewide & $S$ & 7.55 & 6.73 & 6.12 \\
\hline 299-E28-6 & WHC & ES\&M (ROM) & $\mathrm{B}$ & 13.03 & 12.21 & 11.61 \\
\hline 299-E28-7 & WHC & ES\&M (ROM) & $\bar{B}$ & 15.83 & 15.01 & 14.4 \\
\hline 299-E28-8 & WHC & ES\&M (ROM) & $\mathrm{B}$ & 8.7 & 7.88 & 7.28 \\
\hline 299-E28-9 & WHC & ES\&M (ROM) & B & 12.84 & 12.01 & 11.41 \\
\hline 299-E32-1 & WHC & ES\&M (ROM) & $\mathrm{B}$ & 5.35 & 4.53 & 3.93 \\
\hline 299-E32-10 & WHC & ES\&M (ROM) & $\mathrm{H}$ & 3.08 & 2.26 & 1.66 \\
\hline 299-E32-2 & WHC & ES\&M (ROM) & $\mathrm{H}$ & 3.19 & 2.36 & 1.76 \\
\hline 299-E32-3 & WHC & ES\&M (ROM) & $\mathrm{H}$ & 3.86 & 3.03 & 2.43 \\
\hline 299-E32-4 & WHC & ES\&M (ROM) & $\mathrm{H}$ & 4.58 & 3.76 & 3.16 \\
\hline 299-E32-5 & WHC & ES\&M (ROM) & $\mathrm{H}$ & 3.81 & 2.99 & 2.39 \\
\hline 299-E32-6 & WHC & ES\&M (ROM) & $\mathrm{H}$ & 3.29 & 2.47 & 1.87 \\
\hline 299-E32-7 & WHC & ES\&M (ROM) & $\mathrm{H}$ & 3.33 & 2.5 & 1.9 \\
\hline 299-E32-8 & WHC & ES\&M (ROM) & $\mathrm{H}$ & 3.79 & 2.96 & 2.37 \\
\hline 299-E32-9 & WHC & ES\&M (ROM) & $\mathrm{H}$ & 3.26 & 2.43 & 1.84 \\
\hline 299-E33-10 & $\overline{\mathrm{PNL}}$ & Sitewide & $S$ & 6.59 & 5.77 & 5.17 \\
\hline 299-E33-13 & WHC & ES\&M (ROM) & $\mathrm{B}$ & 3.74 & 2.91 & 2.31 \\
\hline 299-E33-14 & WHC & ES\&M (ROM) & $\mathrm{H}$ & 2.37 & 1.54 & 0.94 \\
\hline 299-E33-15 & WHC & ES\&M (ROM) & $\mathrm{B}$ & 3.81 & 2.99 & 2.39 \\
\hline 299-E33-16 & WHC & ES\&M (ROM) & & 4.05 & 3.23 & 2.63 \\
\hline 299-E33-17 & WHC & ES\&M (ROM) & B & 4.62 & 3.8 & 3.2 \\
\hline
\end{tabular}




\begin{tabular}{|c|c|c|c|c|c|c|}
\hline 299-E33-18 & WHC & ES\&M (ROM) & $S$ & 3.34 & 2.51 & 1.91 \\
\hline 299-E33-19 & WHC & ES\&M (ROM) & & 3.69 & 2.86 & 2.26 \\
\hline 299-E33-2 & WHC & ES\&M (ROM) & $\bar{B}$ & 1.83 & 1.01 & 0.41 \\
\hline 299-E33-20 & WHC & ES\&M (ROM) & B & 3.97 & 3.14 & 2.54 \\
\hline 299-E33-21 & WHC & ES\&M (ROM) & $S$ & 2.95 & 2.12 & 1.52 \\
\hline 299-E33-22 & WHC & $\overline{\mathrm{ES} \& \mathrm{M}(\mathrm{ROM})}$ & & 1.52 & 0.7 & 0.1 \\
\hline 299-E33-23 & WHC & ES\&M (ROM) & & 1.38 & 0.56 & -0.04 \\
\hline 299-E33-25 & WHC & ES\&M (ROM) & B & 1.44 & 0.61 & 0.01 \\
\hline 299-E33-26 & WHC & ES\&M (ROM) & $\overline{\mathrm{H}}$ & 2.12 & 1.3 & 0.7 \\
\hline 299-E33-28 & WHC & ES\&M (ROM) & $\mathrm{H}$ & 4.34 & 3.51 & 2.91 \\
\hline 299-E33-29 & WHC & ES\&M (ROM) & $\overline{\mathrm{H}}$ & 3.6 & 2.77 & 2.17 \\
\hline 299-E33-3 & $\overline{\text { WHC }}$ & ES\&M (ROM) & $S$ & 2.46 & 1.63 & 1.03 \\
\hline 299-E33-30 & WHC & ES\&M (ROM) & $\overline{\mathrm{H}}$ & 4.29 & 3.46 & 2.86 \\
\hline 299-E33-31 & WHC & ES\&M (ROM) & $\overline{\mathrm{H}}$ & 3.47 & 2.65 & 2.05 \\
\hline 299-E33-32 & WHC & $\overline{\mathrm{ES} \& \mathrm{M}(\mathrm{ROM})}$ & $\mathrm{H}$ & 3.16 & 2.33 & 1.73 \\
\hline 299-E33-33 & WHC & ES\&M (ROM) & $\overline{\mathrm{H}}$ & $3: 34$ & 2.51 & 1.91 \\
\hline 299-E33-34 & WHC & ES\&M (ROM) & $\overline{\mathrm{H}}$ & 2.56 & 1.73 & 1.13 \\
\hline 299-E33-35 & WHC & ES\&M (ROM) & $\mathrm{H}$ & 2.66 & 1.84 & 1.24 \\
\hline 299-E33-36 & WHC & ES\&M (ROM) & $\mathrm{H}$ & 3.52 & 2.7 & 2.09 \\
\hline 299-E33-37 & WHC & ES\&M (ROM) & $\overline{\mathrm{H}}$ & 3.33 & 2.5 & 1.9 \\
\hline 299-E33-38 & WHC & ES\&M (ROM) & $\overline{\mathrm{H}}$ & 3.17 & 2.35 & 1.75 \\
\hline 299-E33-39 & WHC & ES\&M (ROM) & $\overline{\mathrm{H}}$ & 2.65 & 1.82 & 1.22 \\
\hline 299-E33-4 & WHC & ES\&M (ROM) & B & 1.79 & 0.97 & 0.37 \\
\hline 299-E33-41 & WHC & ES\&M (ROM) & $\mathrm{H}$ & 2.7 & 1.88 & 1.27 \\
\hline 299-E33-42 & $\overline{\mathrm{WHC}}$ & ES\&M (ROM) & $\vec{H}$ & 2.43 & 1.61 & 1.01 \\
\hline 299-E33-43 & WHC & ES\&M (ROM) & $\overline{\mathrm{H}}$ & 3.49 & 2.67 & 2.07 \\
\hline 299-E33-5 & WHC & ES\&M (ROM) & $\bar{S}$ & 1.8 & 0.98 & 0.38 \\
\hline 299-E33-6 & WHC & ES\&M (ROM) & $\bar{B}$ & 1.19 & 0.36 & -0.24 \\
\hline 299-E33-7 & WHC & ES\&M (ROM) & $\bar{B}$ & 1.88 & 1.05 & 0.45 \\
\hline 299-E33-8 & WHC & ES\&M (ROM) & $S$ & 2.76 & 1.93 & 1.33 \\
\hline 299-E33-9 & WHC & $\begin{array}{l}\text { TWRS } \\
\end{array}$ & $B$ & 4.32 & 3.49 & 2.89 \\
\hline 299-E34-1 & WHC & ES\&M (ROM) & $\mathrm{H}$ & 1.03 & 0.2 & -0.4 \\
\hline 299-E34-10 & WHC & ES\&M (ROM) & $\overline{\mathrm{H}}$ & 2.87 & 2.04 & 1.44 \\
\hline 299-E34-11 & WHC & ES\&M (ROM) & $\mathrm{H}$ & 0.83 & 0.01 & -0.59 \\
\hline 299-E34-12 & WHC & ES\&M (ROM) & $\overline{\mathrm{H}}$ & 2.59 & 1.76 & 1.16 \\
\hline 299-E34-2 & WHC & ES\&M (ROM) & $\overline{\mathrm{H}}$ & 3.74 & 2.92 & 2.31 \\
\hline 299-E34-3 & WHC & ES\&M (ROM) & $\mathrm{H}$ & 1.47 & 0.64 & 0.03 \\
\hline 299-E34-7 & WHC & ES\&M (ROM) & $\overline{\mathrm{H}}$ & 0.96 & 0.13 & -0.48 \\
\hline 299-E34-8 & WHC & ES\&M (ROM) & $\overline{\mathrm{H}}$ & 3.12 & 2.29 & 1.69 \\
\hline 299-E34-9 & WHC & ES\&M (ROM) & $\mathrm{H}$ & 2.39 & 1.56 & 0.96 \\
\hline 299-E35-1 & WHC & ES\&M (ROM) & $\overline{\mathrm{H}}$ & -1.02 & -1.85 & -2.46 \\
\hline 299-E35-2 & WHC & ES\&M (ROM) & $\overline{\mathrm{H}}$ & 0.68 & -0.15 & -0.75 \\
\hline $299-\mathrm{W} 10-1$ & WHC & ES\&M (ROM) & $\bar{B}$ & 17.24 & 16.6 & 16.04 \\
\hline 299-W10-10 & WHC & ES\&M (ROM) & $\mathrm{B}$ & 9.47 & 8.87 & 8.32 \\
\hline 299-W10-11 & WHC & ES\&M (ROM) & $\mathrm{B}$ & 9.41 & 8.81 & 8.27 \\
\hline 299-W10-12 & WHC & ES\&M (ROM) & $\bar{B}$ & 9.57 & 8.97 & 8.42 \\
\hline
\end{tabular}




\begin{tabular}{|c|c|c|c|c|c|c|}
\hline 299-W10-13 & WHC & ES\&M (ROM) & $\overline{\mathrm{H}}$ & 2.59 & 1.88 & 1.31 \\
\hline 299-W10-14 & WHC & ES\&M (ROM) & $\overline{\mathrm{H}}$ & 63.44 & 62.73 & 62.16 \\
\hline 299-W10-15 & $\overline{\mathrm{WHC}}$ & ES\&M (ROM) & $\overline{\mathrm{H}}$ & 1.34 & 0.74 & 0.2 \\
\hline $299-W 10-16$ & WHC & ES\&M (ROM) & $\overline{\mathrm{H}}$ & 1.53 & 0.8 & 0.21 \\
\hline 299-W10-17 & WHC & $\mathrm{ES} \& \mathrm{M}(\mathrm{ROM})$ & $\mathrm{H}$ & 3.23 & 2.44 & 1.83 \\
\hline 299-W10-18 & WHC & ES\&M (ROMM) & $\mathrm{H}$ & 2.69 & 1.85 & 1.22 \\
\hline 299-W10-19 & WHC & ES\&M (ROM) & $\overline{\mathrm{H}}$ & 3.22 & 2.77 & 2.28 \\
\hline 299-W10-2 & WHC & ES\&M (ROM) & $\overline{\mathrm{H}}$ & 4.21 & 3.49 & 2.91 \\
\hline 299-W10-3 & WHC & ES\&M (ROM) & B & 5.19 & 4.51 & 3.93 \\
\hline 299-W10-4 & $\overline{\mathrm{WHC}}$ & ES\&M (ROM) & $\bar{S}$ & 10.06 & 9.32 & 8.73 \\
\hline 299-W10-5 & WHC & ES\&M (ROM) & $\bar{B}$ & 2.15 & 1.37 & 0.76 \\
\hline 299-W10-8 & WHC & ES\&M (ROM) & $\overline{\mathrm{B}}$ & 8.78 & 8.17 & 7.62 \\
\hline 299-W10-9 & WHC & ES\&M (ROM) & $S$ & 1.07 & 0.47 & -0.08 \\
\hline 299-W11-1 & $\overline{\mathrm{PNL}}$ & Sitewide & & 18.55 & 17.79 & 17.19 \\
\hline 299-W11-10 & WHC & $\mathrm{ES \& M}(\mathrm{ROM})$ & $\bar{B}$ & 7.12 & 6.46 & 5.88 \\
\hline 299-W11-11 & WHC & ES\&M (ROM) & $\bar{S}$ & 10.21 & 9.4 & 8.77 \\
\hline 299-W11-12 & WHC & ES\&M (ROM) & $\bar{B}$ & 8.78 & 8.03 & 7.43 \\
\hline 299-W11-13 & $\overline{\mathrm{PNL}}$ & Sitewide & $\bar{B}$ & 72.2 & 71.36 & 70.71 \\
\hline 299-W11-130 & PNL & Sitewide & & 4.76 & 3.92 & 3.27 \\
\hline 299-W11-13P & PNL & Sitewide & & 71.2 & 70.35 & 69.71 \\
\hline 299-W11-13Q & $\overline{\mathrm{PNL}}$ & Sitewide & & 50.78 & 49.94 & 49.29 \\
\hline 299-W11-14 & PNL & Sitewide & B & 15.34 & 14.72 & 14.2 \\
\hline 299-W11-15 & PNL & Sitewide & $S$ & 3.11 & 2.78 & 2.35 \\
\hline 299-W11-16 & WHC & ES\&M (ROM) & & 32.23 & 31.86 & 31.42 \\
\hline 299-W11-17 & PNL & Sitewide & & 13.82 & 13.43 & 12.97 \\
\hline 299-W11-18 & PNL & Sitewide & $S$ & 13.03 & 12.72 & 12.3 \\
\hline 299-W11-19 & WHC & ES\&M (ROM) & $\mathrm{B}$ & 34.34 & 34.01 & 33.59 \\
\hline 299-W11-2 & PNL & Sitewide & $\mathrm{B}$ & 75.92 & 75.17 & 74.57 \\
\hline 299-W11-21 & $\overline{\mathrm{PNL}}$ & Sitewide & & 4.07 & 3.74 & 3.31 \\
\hline 299-W11-23 & $\overline{\mathrm{WHC}}$ & ES\&M (ROM) & $S$ & 3.74 & 3.13 & 2.57 \\
\hline 299-W11-24 & WHC & ES\&M (ROM) & $\bar{S}$ & 0.19 & -0.47 & -1.03 \\
\hline 299-W11-27 & WHC & ES\&M (ROM) & $\overline{\mathrm{H}}$ & 1.85 & 1.24 & 0.68 \\
\hline 299-W11-28 & WHC & ES\&M (ROM) & $\mathrm{H}$ & 2.79 & 2.13 & 1.56 \\
\hline 299-W11-29 & PNL & Sitewide & & 8.03 & 7.43 & 6.88 \\
\hline 299-W11-20 & $\mathrm{PNL}$ & Sitewide & & 0.02 & -0.72 & -1.32 \\
\hline 299-W11-2P & PNL & Sitewide & & 75.92 & 75.17 & 74.57 \\
\hline 299-W11-2Q & $\mathrm{PNL}$ & Sitewide & & 53.36 & 52.62 & 52.02 \\
\hline 299-W11-2R & $\mathrm{PNL}$ & Sitewide & & 36.9 & 36.16 & 35.56 \\
\hline 299-W11-2S & PNL & Sitewide & & 19.22 & 18.48 & 17.88 \\
\hline 299-W11-2T & PNL & Sitewide & & 1.55 & 0.8 & 0.2 \\
\hline 299-W11-3 & WHC & ES\&M (ROM) & $B$ & 16.8 & 16.07 & 15.49 \\
\hline 299.W11-30 & $\mathrm{PNL}$ & Sitewide & $\mathrm{B}$ & 8.23 & 7.63 & 7.09 \\
\hline 299-W11-31 & WHC & ES\&M (ROM) & $\mathrm{H}$ & 2.63 & 2.4 & 2.02 \\
\hline 299-W11-4 & PNL & Sitewide & & 13.19 & 12.4 & 11.77 \\
\hline 299-W11-5 & WHC & ES\&M (ROM) & & 15.3 & 14.51 & 13.9 \\
\hline 299-W11-6 & WHC & ES\&M (ROM) & $\mathrm{B}$ & 15.78 & 14.94 & 14.3 \\
\hline
\end{tabular}




\begin{tabular}{|c|c|c|c|c|c|c|}
\hline 299-W11-7 & WHC & ES\&M (ROM) & $B$ & 11.53 & 10.78 & 10.18 \\
\hline 299-W11-8 & WHC & ES\&M (ROM) & & 13.78 & 12.96 & 12.34 \\
\hline 299-W11-9 & WHC & ES\&M (ROM) & $\mathrm{B}$ & 6.47 & 5.75 & 5.19 \\
\hline $299-W 12-1$ & WHC & ES\&M (ROM) & $B$ & 8.03 & 7.62 & 7.2 \\
\hline 299-W14-1 & WHC & ES\&M (ROM) & $\bar{B}$ & 6.92 & 6.04 & 5.38 \\
\hline 299-W14-12 & WHC & ES\&M (ROM) & $\mathrm{H}$ & 2.11 & 1.22 & 0.55 \\
\hline 299-W14-2 & PNL & Sitewide & $\mathbf{S}$ & 4.01 & 3.15 & 2.49 \\
\hline $299-W 14-3$ & WHC & ES\&M (ROM) & & 19.67 & 18.8 & 18.15 \\
\hline 299-W14-5 & WHC & ES\&M (ROM) & $S$ & 5.36 & 4.36 & 3.66 \\
\hline 299-W14-6 & WHC & ES\&M (ROM) & $S$ & 5.97 & 5 & 4.3 \\
\hline 299-W15-1 & WHC & ES\&M (ROM) & & 17.53 & 16.52 & 15.81 \\
\hline 299-W15-10 & $\overline{\mathrm{WHC}}$ & ES\&M (ROM) & $S$ & 24.88 & 23.89 & 23.19 \\
\hline 299-W15-11 & $\overline{\mathrm{WHC}}$ & ES\&M (ROM) & $S$ & 24.6 & 23.62 & 22.92 \\
\hline 299-W15-12 & WHC & ES\&M (ROM) & $\bar{B}$ & 1.26 & 0.42 & -0.22 \\
\hline 299-W15-13 & WHC & ES\&M (ROM) & & 4.16 & 3.31 & 2.66 \\
\hline 299-W15-15 & WHC & ES\&M (ROM) & $\mathrm{H}$ & 5.15 & 4.12 & $\overline{3.41}$ \\
\hline 299-W15-16 & WHC & ES\&M (ROM) & $\mathrm{H}$ & 4.43 & 3.37 & 2.63 \\
\hline 299-W15-17 & WHC & ES\&M (ROM) & $\mathrm{H}$ & 63.8 & 62.73 & 61.99 \\
\hline $299-W 15-18$ & WHC & ES\&M (ROM) & $\overline{\mathrm{H}}$ & 4.21 & 3.1 & 2.35 \\
\hline 299-W15-19 & WHC & ES\&M (ROM) & $\overline{\mathrm{H}}$ & 1.39 & 0.42 & -0.27 \\
\hline 299-W15-2 & WHC & ES\&M (ROM) & $\mathrm{B}$ & 7.74 & 6.91 & 6.29 \\
\hline 299-W15-20 & WHC & ES\&M (ROM) & $\overline{\mathrm{H}}$ & 1.31 & 0.36 & -0.32 \\
\hline 299-W15-22 & WHC & ES\&M (ROM) & $\overline{\mathrm{H}}$ & 2.67 & 1.71 & 1.02 \\
\hline 299-W15-23 & WHC & ES\&M (ROM) & $\overline{\mathrm{H}}$ & 0.5 & -0.49 & -1.19 \\
\hline 299-W15-24 & WHC & ES\&M (ROM) & $\mathrm{H}$ & 1.1 & 0.03 & -0.71 \\
\hline 299-W15-3 & WHC & ES\&M (ROM) & $\bar{B}$ & 10.24 & 9.4 & 8.76 \\
\hline 299-W15-4 & WHC & ES\&M (ROM) & $\mathbf{S}$ & 4.1 & 3.1 & 2.39 \\
\hline 299-W15-5 & WHC & ES\&M (ROM) & $B$ & 1.96 & 0.82 & 0.05 \\
\hline 299-W15-50 & WHC & ES\&M (ROM) & & 2.79 & 1.65 & 0.88 \\
\hline 299-W15-5P & WHC & ES\&M (ROM) & & 116.79 & 115.64 & 114.87 \\
\hline 299-W15-5Q & WHC & ES\&M (ROM) & & 94.23 & 93.09 & 92.32 \\
\hline 299-W15-5R & WHC & ES\&M (ROM) & & 75.95 & 74.8 & 74.03 \\
\hline 299-W15-5S & WHC & ES\&M (ROM) & & 57.66 & 56.51 & 55.74 \\
\hline 299-W15-7 & PNL & Sitewide & $S$ & 44.52 & 43.49 & 42.77 \\
\hline 299-W18-1 & $\overline{\mathrm{PNL}}$ & Sitewide & $S$ & 6.44 & 5.31 & 4.55 \\
\hline 299-W18-10 & WHC & ES\&M (ROM) & $\underline{S}$ & -1.14 & -2.3 & -3.08 \\
\hline 299-W18-11 & WHC & ER & & -1.22 & -2.37 & -3.15 \\
\hline 299-W18-12 & WHC & $\overline{E R}$ & $S$ & -2.43 & -3.58 & -4.36 \\
\hline 299-W18-15 & WHC & ES\&M (ROM) & $S$ & 13.13 & 11.95 & 11.14 \\
\hline 299-W18-2 & WHC & ER & B & 10.74 & 9.61 & 8.84 \\
\hline 299-W18-21 & WHC & ES\&M (ROM) & $\mathrm{H}$ & 5.71 & 4.57 & 3.79 \\
\hline 299-W18-22 & WHC & ES\&M (ROM) & $\mathrm{H}$ & 73.42 & 72.29 & 71.5 \\
\hline 299-W18-23 & WHC & ES\&M (ROM) & $\mathrm{H}$ & 4.92 & 3.82 & 3.06 \\
\hline 299-W18-24 & WHC & ES\&M (ROM) & $\mathrm{H}$ & 3.78 & 2.64 & 1.87 \\
\hline 299-W18-25 & WHC & ES\&M (ROM) & $\mathrm{H}$ & 2.7 & 1.48 & 0.66 \\
\hline 299-W18-26 & WHC & ES\&M (ROM) & $\mathrm{H}$ & 1.9 & 0.81 & 0.06 \\
\hline
\end{tabular}




\begin{tabular}{|c|c|c|c|c|c|c|}
\hline 299-W18-27 & WHC & $\mathrm{ES} \& \mathrm{M}(\mathrm{ROM})$ & $\mathrm{H}$ & 2.86 & 1.74 & 0.97 \\
\hline 299-W18-28 & WHC & ES\&M (ROM) & $\overline{\mathrm{H}}$ & 3.39 & 2.26 & 1.48 \\
\hline 299-W18-3 & $\overline{\mathrm{WHC}}$ & ES\&M (ROM) & $\mathrm{B}$ & 6.78 & 5.69 & 4.94 \\
\hline 299-W18-30 & $\overline{W H C}$ & ES\&M (ROM) & $\overline{\mathrm{H}}$ & $6 . \overline{43}$ & 5.23 & 4.42 \\
\hline 299-W18-31 & WHC & ES\&M (ROM) & $\overline{\mathrm{H}}$ & 5.57 & 4.36 & 3.55 \\
\hline 299-W18-32 & WHC & ES\&M (ROM) & $\mathrm{H}$ & 1.88 & 0.69 & -0.11 \\
\hline 299-W18-33 & $\overline{\mathrm{WHC}}$ & $\overline{\mathrm{ES} \& \mathrm{M}(\mathrm{ROM})}$ & $\mathbf{S}$ & 5.89 & 4.67 & 3.85 \\
\hline 299-W18-4 & PNL & Sitewide & $\bar{B}$ & 8.98 & 7.85 & 7.09 \\
\hline 299-W18-5 & PNL & Sitewide & $\mathbf{S}$ & 15.96 & 14.84 & 14.07 \\
\hline 299-W18-6 & WHC & ER & $\mathrm{B}$ & 24.49 & 23.35 & 22.58 \\
\hline 299-W18-7 & $\overline{\mathrm{WHC}}$ & ER & $\bar{B}$ & 24.27 & 23.12 & 22.35 \\
\hline 299-W18-9 & $\overline{\mathrm{WHC}}$ & ER & $\overline{\mathrm{B}}$ & -1.08 & -2.23 & -3.01 \\
\hline 299-W19-1 & WHC & ES\&M (ROM) & $\overline{\mathrm{B}}$ & 25.83 & 24.61 & 23.78 \\
\hline 299-W19-11 & WHC & ES\&M (ROM) & $S$ & 3.75 & 2.53 & 1.7 \\
\hline 299-W19-12 & $\overline{\mathrm{WHC}}$ & ES\&M (ROM) & $S$ & 11.05 & 9.84 & 9.03 \\
\hline 299-W19-13 & PNL & Sitewide & $S$ & 3.87 & 2.66 & 1.81 \\
\hline 299-W19-14 & WHC & ES\&M (ROM) & $\bar{S}$ & 4.42 & 3.21 & 2.36 \\
\hline 299-W19-15 & $\overline{\mathrm{WHC}}$ & ES\&M (ROM) & $\bar{S}$ & 12.13 & 10.92 & 10.08 \\
\hline 299-W19-16 & $\overline{\text { WHC }}$ & ES\&M (ROM) & $\mathbf{S}$ & 10.08 & 8.87 & 8.04 \\
\hline 299-W19-17 & WHC & ES\&M (ROM) & $\bar{S}$ & 34.77 & 33.56 & 32.73 \\
\hline 299-W19-18 & WHC & ES\&M (ROM) & $\mathbf{S}$ & 34.73 & 33.51 & 32.67 \\
\hline 299-W19-19 & WHC & ES\&M (ROM) & $\mathbf{S}$ & 2.95 & 1.79 & 0.93 \\
\hline 299-W19-2 & $\overline{\mathrm{WHC}}$ & ES\&M (ROM) & 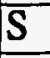 & 17.11 & 15.93 & 15.07 \\
\hline 299-W19-20 & WHC & ES\&M (ROM) & $\mathbf{S}$ & 4.36 & 3.2 & $2 . \overline{34}$ \\
\hline 299-W19-21 & WHC & ES\&M (ROM) & $\mathbf{S}$ & 2.05 & 0.83 & 0 \\
\hline 299-W19-23 & WHC & ES\&M (ROM) & $\bar{S}$ & 3.47 & 2.3 & 1.44 \\
\hline 299-W19-24 & WHC & ES\&M (ROM) & $S$ & 2.73 & 1.56 & 0.7 \\
\hline 299-W19-25 & WHC & ES\&M (ROM) & $S$ & 3.27 & 2.11 & 1.25 \\
\hline 299-W19-26 & WHC & ES\&M (ROM) & $S$ & 3.31 & 2.15 & 1.29 \\
\hline 299-W19-27 & WHC & ES\&M (ROM) & $S$ & 1.04 & -0.17 & -1 \\
\hline 299-W19-28 & WHC & ES\&M (ROM) & $\overline{\mathrm{H}}$ & 2.71 & 1.51 & 0.66 \\
\hline 299-W19-29 & WHC & ES\&M (ROM) & $\mathrm{H}$ & 2.63 & 1.44 & 0.59 \\
\hline 299-W19-3 & WHC & ES\&M (ROM) & $\overline{\mathrm{H}}$ & 12.24 & 11.03 & 10.19 \\
\hline 299-W19-30 & PNL & Sitewide & $\overline{\mathrm{H}}$ & 2.9 & 1.72 & 0.87 \\
\hline 299-W19-31 & WHC & ES\&M (ROM) & $\mathrm{H}$ & 2.42 & 1.22 & 0.4 \\
\hline 299-W19-32 & WHC & ES\&M (ROM) & $\overline{\mathrm{H}}$ & 2.12 & 0.91 & 0.09 \\
\hline 299-W19-4 & WHC & ES\&M (ROM) & $\bar{B}$ & 48.69 & 47.57 & 46.73 \\
\hline 299-W19-5 & WHC & ES\&M (ROM) & $\bar{B}$ & -3.6 & -4.81 & -5.66 \\
\hline 299-W19-6 & WHC & ES\&M (ROM) & $\mathrm{B}$ & 55.65 & 54.44 & 53.59 \\
\hline 299-W19-7 & $\overline{W H C}$ & ES\&M (ROM) & & -2.98 & -4.18 & -5.03 \\
\hline 299-W19-9 & WHC & ES\&M (ROM) & $\mathbf{S}$ & 66.45 & 65.24 & 64.41 \\
\hline 299-W21-1 & WHC & ES\&M (ROM) & $\mathrm{B}$ & 13.06 & 11.94 & 11.06 \\
\hline 299-W22-1 & WHC & ES\&M (ROM) & $S$ & 21.15 & 19.95 & 19.1 \\
\hline $299-W 22-10$ & PNL & Sitewide & $\mathrm{B}$ & 29.47 & 28.27 & 27.42 \\
\hline 299-W22-11 & WHC & ES\&M (ROM) & $S$ & 29.12 & 27.91 & 27.06 \\
\hline 299-W22-12 & PNL & Sitewide & $\mathrm{B}$ & 4.56 & 3.37 & 2.51 \\
\hline
\end{tabular}




\begin{tabular}{|c|c|c|c|c|c|c|}
\hline 299-W22-13 & WHC & ES\&M (ROM) & & 36.19 & 35 & 34.14 \\
\hline 299-W22-14 & WHC & ES\&M (ROM) & & 36.25 & 35.07 & 34.2 \\
\hline 299-W22-15 & WHC & ES\&M (ROM) & & 15.58 & 14.38 & 13.52 \\
\hline 299-W22-16 & WHC & ES\&M (ROM) & & 9.77 & 8.57 & 7.71 \\
\hline 299-W22-17 & WHC & ES\&M (ROM) & B & 14.13 & 12.93 & 12.07 \\
\hline 299-W22-18 & WHC & ES\&M (ROM) & $\bar{B}$ & 26.32 & 25.12 & 24.26 \\
\hline 299-W22-19 & WHC & ES\&M (ROM) & B & 51.19 & 50.06 & 49.18 \\
\hline $299-W 22-2$ & PNL & Sitewide & $\mathrm{B}$ & 22.83 & 21.63 & 20.77 \\
\hline 299-W22-20 & PNL & Sitewide & $S$ & 23.46 & 22.33 & 21.45 \\
\hline 299-W22-21 & WHC & ES\&M (ROM) & $\overline{\mathrm{B}}$ & 21.85 & 20.69 & 19.82 \\
\hline 299-W22-22 & $\overline{\mathrm{WHC}}$ & ES\&M (ROM) & $\mathrm{H}$ & 19.77 & 18.6 & 17.73 \\
\hline 299-W22-23 & WHC & ES\&M (ROM) & & 19.62 & 18.45 & 17.57 \\
\hline 299-W22-24 & WHC & ES\&M (ROM) & B & 98.26 & 97.1 & 96.22 \\
\hline 299-W22-24O & WHC & ES\&M (ROM) & & -2.39 & -3.56 & -4.43 \\
\hline 299-W22-24P & WHC & ES\&M (ROM) & & 98.5 & 97.34 & 96.47 \\
\hline 299-W22-24Q & WHC & ES\&M (ROM) & & 79 & 77.84 & 76.96 \\
\hline 299-W22-24R & WHC & ES\&M (ROM) & & 60.71 & 59.54 & 58.67 \\
\hline $299-\mathrm{W} 22-24 \mathrm{~S}$ & WHC & ES\&M (ROM) & & 42.43 & 41.26 & 40.39 \\
\hline 299-W22-24T & WHC & ES\&M (ROM) & & 24.14 & 22.97 & 22.1 \\
\hline $299-\mathrm{W} 22-25$ & WHC & ES\&M (ROM) & & 22.69 & 21.5 & 20.64 \\
\hline $299-W 22-26$ & WHC & ES\&M (ROM) & $S$ & 22.87 & 21.67 & 20.81 \\
\hline 299-W22-27 & WHC & ES\&M (ROM) & & 20.42 & 19.21 & 18.36 \\
\hline 299-W22-27P & WHC & ES\&M (ROM) & & 105.96 & 104.76 & 103.9 \\
\hline 299-W22-27Q & WHC & ES\&M (ROM) & & 81.58 & 80.37 & 79.52 \\
\hline 299-W22-27R & WHC & ES\&M (ROM) & & 60.24 & 59.04 & 58.18 \\
\hline 299-W22-27S & WHC & ES\&M (ROM) & & 38.9 & $\mid 37.7$ & 36.85 \\
\hline 299-W22-28 & WHC & ES\&M (ROM) & $\mathrm{B}$ & 19.52 & 18.34 & 17.47 \\
\hline $299-W 22-3$ & WHC & ES\&M (ROM) & & 22.25 & 21.05 & 20.2 \\
\hline 299-W22-37 & WHC & ES\&M (ROM) & & 1.35 & \begin{tabular}{|l|}
0.13 \\
\end{tabular} & -0.71 \\
\hline 299-W22-38 & WHC & ES\&M (ROM) & & 1.54 & 0.33 & -0.52 \\
\hline 299-W22-39 & WHC & ES\&M (ROM) & $\mathrm{H}$ & 3.46 & 2.26 & 1.41 \\
\hline 299-W22-4 & WHC & ES\&M (ROM) & & 30.01 & 28.81 & 27.95 \\
\hline 299-W22-40 & WHC & ES\&M (ROM) & $\mathrm{H}$ & 2.17 & 1 & 0.13 \\
\hline 299-W22-41 & WHC & ES\&M (ROM) & $\overline{\mathrm{H}}$ & 2.56 & 1.4 & 0.52 \\
\hline $299-W 22-42$ & WHC & ES\&M (ROM) & $\mathrm{H}$ & 2.16 & 1 & 0.12 \\
\hline $299-W 22-43$ & WHC & ES\&M (ROM) & $\mathrm{H}$ & 2.47 & 1.29 & 0.42 \\
\hline 299-W22-44 & WHC & ES\&M (ROM) & $\overline{\mathrm{H}}$ & 6.92 & 5.71 & 4.86 \\
\hline 299-W22-45 & WHC & ES\&M (ROM) & $\overline{\mathrm{H}}$ & 7.93 & 6.72 & 5.87 \\
\hline 299-W22-46 & WHC & ES\&M (ROM) & $\mathrm{H}$ & 4.85 & 3.66 & 2.8 \\
\hline $299-W 22-5$ & WHC & ES\&M (ROM) & & 31.18 & 29.99 & 29.13 \\
\hline 299-W22-6 & WHC & ES\&M (ROM) & & 18.79 & 17.63 & 16.75 \\
\hline 299-W22-7 & WHC & ES\&M (ROM) & B & 3.1 & 1.93 & 1.05 \\
\hline 299-W22-8 & WHC & ES\&M (ROM) & $\mathrm{B}$ & 16.31 & 15.16 & 14.28 \\
\hline 299-W22-9 & PNL & Sitewide & $\mathrm{B}$ & 5.79 & 4.64 & 3.77 \\
\hline 299-W23-1 & WHC & ES\&M (ROM) & $\bar{B}$ & 8.31 & 7.11 & 6.27 \\
\hline 299-W23-10 & PNL & Sitewide & $S$ & 7.6 & 6.4 & 5.56 \\
\hline
\end{tabular}




\begin{tabular}{|c|c|c|c|c|c|c|}
\hline 299-W23-11 & WHC & ES\&M (ROM) & S & 0.87 & -0.33 & -1.16 \\
\hline 299-W23-12 & WHC & ES\&M (ROM) & & 6.94 & 5.74 & 4.89 \\
\hline 299-W23-13 & WHC & ES\&M (ROM) & $\overline{\mathrm{H}}$ & 3.15 & 1.94 & 1.1 \\
\hline 299-W23-14 & WHC & ES\&M (ROM) & $\overline{\mathrm{H}}$ & 3.19 & 1.99 & 1.15 \\
\hline 299-W23-15 & WHC & ES\&M (ROM) & $\bar{H}$ & 7.81 & 6.63 & 5.77 \\
\hline 299-W23-16 & WHC & ES\&M (ROM) & & 6.02 & 4.81 & 3.99 \\
\hline 299-W23-17 & WHC & ES\&M (ROM) & & 5.6 & 4.4 & 3.57 \\
\hline 299-W23-2 & WHC & ES\&M (ROM) & $\mathrm{B}$ & 9.18 & 7.98 & 7.13 \\
\hline 299-W23-3 & WHC & ES\&M (ROM) & $\mathrm{B}$ & 6.98 & 5.79 & 4.93 \\
\hline 299-W23-4 & WHC & ES\&M (ROM) & $\overline{\mathrm{B}}$ & 29.59 & 28.38 & 27.55 \\
\hline 299-W23-5 & WHC & TWRS & & 11.97 & 10.77 & 9.92 \\
\hline 299-W23-6 & $\overline{\text { WHC }}$ & ES\&M (ROM) & $\bar{B}$ & 12.06 & 10.87 & 10.02 \\
\hline 299-W23-7 & $\overline{\mathrm{WHC}}$ & ES\&M (ROM) & $\overline{\mathrm{B}}$ & 13.11 & 11.91 & 11.06 \\
\hline 299-W23-8 & $\overline{\mathrm{WHC}}$ & ES\&M (ROM) & $\bar{B}$ & 7.7 & 6.5 & 5.65 \\
\hline 299-W23-9 & PNL & Sitewide & $\bar{B}$ & 7.6 & 6.41 & 5.56 \\
\hline 299-W26-10 & WHC & ES\&M (ROM) & $\overline{\mathrm{H}}$ & 2.69 & 1.57 & 0.7 \\
\hline 299-W26-11 & $\overline{W H C}$ & ES\&M (ROM) & & -24.9 & -26.04 & -26.91 \\
\hline 299-W26-12 & $\overline{\mathrm{WHC}}$ & ES\&M (ROM) & $\mathrm{H}$ & 2.93 & 1.79 & 0.92 \\
\hline 299-W26-7 & $\overline{\mathrm{WHC}}$ & ES\&M (ROM) & $\overline{\mathrm{H}}$ & 3.84 & 2.76 & 1.89 \\
\hline 299-W26-8 & WHC & ES\&M (ROM) & $\overline{\mathrm{H}}$ & 2.43 & 1.29 & 0.43 \\
\hline 299-W26-9 & $\overline{W H C}$ & ES\&M (ROM) & $\mathrm{H}$ & 2.87 & 1.77 & 0.9 \\
\hline 299-W27-2 & WHC & ES\&M (ROM) & $\mathrm{H}$ & 59.98 & 58.84 & 57.97 \\
\hline 299-W6-1 & WHC & ES\&M (ROM) & $\mathrm{B}$ & 9.09 & 9.12 & 8.85 \\
\hline 299-W6-10 & $\overline{\mathrm{WHC}}$ & ES\&M (ROM) & $\overline{\mathrm{H}}$ & 3.07 & 3 & 2.71 \\
\hline 299-W6-11 & WHC & ES\&M (ROM) & $\overline{\mathrm{H}}$ & 6.22 & 6.37 & 6.15 \\
\hline 299-W6-12 & $\overline{\mathrm{WHC}}$ & ES\&M (ROM) & $\mathrm{H}$ & 5.9 & 6.15 & 5.89 \\
\hline 299-W6-2 & $\overline{\mathrm{WHC}}$ & ES\&M (ROM) & $\mathrm{H}$ & 2.4 & 2.24 & 1.87 \\
\hline 299-W6-3 & WHC & ES\&M (ROM) & $\overline{\mathrm{H}}$ & 53.17 & 52.99 & 52.62 \\
\hline 299-W6-4 & $\overline{\mathrm{WHC}}$ & ES\&M (ROM) & $\mathrm{H}$ & 2.75 & 2.56 & 2.19 \\
\hline 299-W6-5 & $\overline{\text { WHC }}$ & ES\&M (ROM) & $\overline{\mathrm{H}}$ & 6.12 & 6.15 & 5.93 \\
\hline 299-W6-6 & $\overline{\mathrm{WHC}}$ & ES\&M (ROM) & $\overline{\mathrm{H}}$ & 56.54 & 56.66 & 56.44 \\
\hline 299-W6-7 & WHC & ES\&M (ROM) & $\mathrm{H}$ & 7.07 & 7.19 & 6.97 \\
\hline 299-W6-8 & WHC & ES\&M (ROM) & $\mathrm{H}$ & -1.92 & -1.75 & -1.99 \\
\hline 299-W6-9 & WHC & ES\&M (ROM) & $\mathrm{H}$ & 3.13 & 3 & 2.64 \\
\hline 299-W7-1. & WHC & ES\&M (ROM) & $\overline{\mathrm{H}}$ & 3.58 & 3.32 & 2.96 \\
\hline 299-W7-10 & WHC & ES\&M (ROM) & $\mathrm{H}$ & 2.03 & 2.01 & 1.66 \\
\hline 299-W7-11 & WHC & ES\&M (ROM) & $\mathrm{H}$ & 2.31 & 2.12 & 1.77 \\
\hline 299-W7-12 & WHC & ES\&M (ROM) & $\overline{\mathrm{H}}$ & 2.86 & 2.62 & 2.26 \\
\hline 299-W7-2. & WHC & ES\&M (ROM) & $\mathrm{H}$ & 0.99 & 0.83 & 0.48 \\
\hline 299-W7-3 & WHC & ES\&M (ROM) & $\mathrm{H}$ & 76.41 & 76.26 & 75.91 \\
\hline 299-W7-4 & WHC & ES\&M (ROM) & $\overline{\mathrm{H}}$ & 5.81 & 5.51 & 5.08 \\
\hline $299-W 7-5$ & WHC & ES\&M (ROM) & $\overline{\mathrm{H}}$ & 3.41 & 3.46 & 3.13 \\
\hline 299-W7-6 & $\overline{\text { WHC }}$ & ES\&M (ROM) & $\mathrm{H}$ & 2 & 2.27 & 1.97 \\
\hline 299-W7-7 & WHC & ES\&M (ROM) & $\mathrm{H}$ & 2.82 & 2.97 & 2.66 \\
\hline 299-W7-8 & WHC & ES\&M (ROM) & $\mathrm{H}$ & 2.91 & 3.27 & 2.99 \\
\hline 299-W7-9 & WHC & ES\&M (ROM) & $\mathrm{H}$ & 2.03 & 1.74 & 1.38 \\
\hline
\end{tabular}




\begin{tabular}{|c|c|c|c|c|c|c|}
\hline \begin{tabular}{|l|}
$299-W 8-1$ \\
$299-W 9-1$
\end{tabular} & $\begin{array}{l}\text { WHC } \\
\text { WHC }\end{array}$ & \begin{tabular}{|l} 
ES\&M (ROM) \\
ES\&M (ROM)
\end{tabular} & \begin{tabular}{|l}
$\mathrm{H}$ \\
$\mathrm{H}$
\end{tabular} & \begin{tabular}{|l|}
3.96 \\
2.61
\end{tabular} & \begin{tabular}{|l|}
3.66 \\
2.06
\end{tabular} & \begin{tabular}{r|}
3.3 \\
1.56
\end{tabular} \\
\hline $3099-42-16$ & PNL & Sitewide & $\bar{S}$ & 19.93 & 19.83 & 19.82 \\
\hline $399-1-1$ & WHC & ES\&M (ROM) & $S$ & 13.61 & 13.61 & 13.61 \\
\hline $399-1-10 \mathrm{~A}$ & WHC & ES\&M (ROM) & $\mathrm{H}$ & 3.33 & 3.33 & 3.33 \\
\hline 399-1-10B & WHC & ER & $\mathrm{H}$ & 25.75 & 25.75 & 25.75 \\
\hline $399-1-11$ & WHC & ES\&M (ROM) & $\overline{\mathrm{H}}$ & 4.59 & 4.59 & 4.59 \\
\hline $399-1-12$ & WHC & ES\&M (ROM) & $\overline{\mathrm{H}}$ & 6.55 & 6.55 & 6.54 \\
\hline $399-1-13 A$ & WHC & ES\&M (ROM) & $\overline{\mathrm{H}}$ & 3.19 & 3.19 & 3.19 \\
\hline $399-1-13 B$ & WHC & ER & $\overline{\mathrm{H}}$ & 22.7 & 22.7 & 22.7 \\
\hline 399-1-14A & WHC & ES\&M (ROM) & $\overline{\mathrm{H}}$ & 3.01 & 3.01 & \\
\hline $399-1-14 \mathrm{~B}$ & WHC & ER & $\overline{\mathrm{H}}$ & 22.36 & 22.35 & 22.35 \\
\hline $399-1-15$ & WHC & ES\&M (ROM) & $\mathrm{H}$ & 3.18 & 3.18 & 3.17 \\
\hline $399-1-16 \mathrm{~A}$ & WHC & ES\&M (ROM) & $\overline{\mathrm{H}}$ & 3.68 & 3.68 & 3.68 \\
\hline $399-1-16 B$ & WHC & ES\&M (ROM) & $\overline{\mathrm{H}}$ & 24.21 & 24.21 & 24.21 \\
\hline $399-1-16 C$ & WHC & ES\&M (ROM) & $\overline{\mathrm{H}}$ & 42.91 & 42.91 & 42.91 \\
\hline $399-1-17 A$ & WHC & ES\&M (ROM) & $\overline{\mathrm{H}}$ & 2.55 & 2.54 & 2.54 \\
\hline $399-1-17 \mathrm{~B}$ & WHC & ES\&M (ROM) & $\overline{\mathrm{H}}$ & 23.76 & 23.76 & 23.76 \\
\hline $399-1-17 C$ & WHC & ES\&M (ROM) & $\mathrm{H}$ & 42.28 & 42.27 & 42.27 \\
\hline $399-1-18 \mathrm{~A}$ & WHC & ES\&M (ROM) & $\mathrm{H}$ & 2.86 & 2.86 & 2.86 \\
\hline $399-1-18 B$ & WHC & ES\&M (ROM) & $\overline{\mathrm{H}}$ & 22.79 & 22.79 & 22.79 \\
\hline $399-1-18 \mathrm{C}$ & WHC & ES\&M (ROM) & $\overline{\mathrm{H}}$ & 29.77 & 29.77 & 29.76 \\
\hline $399-1-2$ & $\overline{\text { WHC }}$ & ES\&M (ROM) & $\overline{\mathrm{H}}$ & 11.07 & 11.07 & 11.06 \\
\hline $399-1-20$ & PNL & Sitewide & & 19.25 & 19.25 & 19.25 \\
\hline $399-1-21 \mathrm{~A}$ & WHC & ER & $\mathrm{H}$ & 4.73 & 4.72 & 4.72 \\
\hline 399-1-21B & WHC & ER & $\overrightarrow{\mathrm{H}}$ & 22.67 & 22.67 & 22.66 \\
\hline $399-1-3$ & WHC & ES\&M (ROM) & $S$ & 9.42 & 9.42 & 9.42 \\
\hline $399-1-4$ & WHC & ES\&M (ROM) & $S$ & 10.62 & 10.62 & 10.62 \\
\hline $399-1-5$ & WHC & ES\&M (ROM) & $\mathrm{H}$ & 3.36 & 3.36 & 3.36 \\
\hline $399-1-6$ & WHC & ES\&M (ROM) & $\mathbf{S}$ & 4.88 & 4.88 & 4.88 \\
\hline $399-1-7$ & WHC & ES\&M (ROM) & $\overline{\mathrm{H}}$ & 10.67 & 10.66 & 10.66 \\
\hline $399-1-8$ & WHC & ES\&M (ROM) & $\overline{\mathrm{H}}$ & 20.03 & 20.03 & 20.03 \\
\hline $399-1-9$ & WHC & ES\&M (ROM) & $\overline{\mathrm{H}}$ & 42.92 & 42.92 & 42.92 \\
\hline $399-2-1$ & WHC & ES\&M (ROM) & $S$ & 13.72 & 13.72 & 13.72 \\
\hline $399-2-2$ & WHC & $\overline{\mathrm{ES} \& \mathrm{M}(\mathrm{ROM})}$ & $\overline{\mathrm{H}}$ & 7.6 & 7.6 & 7.59 \\
\hline $399-2-3$ & WHC & ES\&M (ROM) & $\mathrm{H}$ & 7.62 & 7.62 & 7.62 \\
\hline $399-3-1$ & WHC & ES\&M (ROM) & $\mathbf{S}$ & 7.87 & 7.87 & 7.87 \\
\hline $399-3-10$ & WHC & ES\&M (ROM) & $\overline{\mathrm{H}}$ & 2.67 & 2.67 & 2.66 \\
\hline $399-3-11$ & WHC & ER & $S$ & 6.05 & 6.05 & 6.05 \\
\hline $399-3-12$ & WHC & ES\&M (ROM) & $S$ & 2.5 & 2.49 & 2.49 \\
\hline 399-3-2 & WHC & ER & $\mathbf{S}$ & 8.73 & 8.72 & 8.72 \\
\hline 399-3-3 & WHC & ER & $S$ & 8.93 & 8.92 & 8.92 \\
\hline 399-3-6 & WHC & ES\&M (ROM) & $\underline{s}$ & 2.47 & 2.46 & 2.46 \\
\hline $399-3-8$ & WHC & ES\&M (ROM) & & 1.84 & 1.84 & 1.83 \\
\hline 399-3-9 & WHC & ES\&M (ROM) & $\underline{S}$ & 3.67 & 3.67 & 3.67 \\
\hline $399-4-1$ & WHC & ES\&M (ROM) & $S$ & 9.09 & 9.08 & 9.08 \\
\hline
\end{tabular}




\begin{tabular}{|c|c|c|c|c|c|c|}
\hline $399-4-10$ & WHC & ES\&M (ROM) & $\mathrm{H}$ & 5.32 & 5.32 & 5.32 \\
\hline $399-4-11$ & $\overline{\mathrm{WHC}}$ & ES\&M (ROM) & $\mathrm{H}$ & 3.41 & 3.4 & 3.39 \\
\hline $399-4-7$ & WHC & ES\&M (ROM) & $S$ & 14.74 & 14.73 & 14.73 \\
\hline $399-4-9$ & $\overline{\mathrm{WHC}}$ & ES\&M (ROM) & $S$ & 6.36 & 6.36 & 6.36 \\
\hline $399-5-1$ & $\overline{\mathrm{WHC}}$ & ES\&M (ROM) & $\mathbf{S}$ & 15.48 & 15.46 & $\overline{15.45}$ \\
\hline $399-6-1$ & $\overline{\mathrm{WHC}}$ & ES\&M (ROM) & $S$ & 6.63 & 6.6 & 6.6 \\
\hline $399-8-1$ & $\overline{\mathrm{WHC}}$ & ES\&M (ROM) & $\overline{\mathrm{H}}$ & 10.12 & 10.11 & 10.11 \\
\hline $399-8-2$ & $\overline{\mathrm{WHC}}$ & ES\&M (ROM) & $\mathrm{H}$ & 14.03 & 14.01 & 14.01 \\
\hline 399-8-3 & WHC & ES\&M (ROM) & $\mathrm{H}$ & 14.43 & 14.42 & 14.41 \\
\hline $399-8-4$ & WHC & ES\&M (ROM) & $\mathbf{S}$ & 4.48 & 4.46 & 4.45 \\
\hline $399-8-5 A$ & WHC & ER & $\mathrm{H}$ & 5.01 & 5 & 4.99 \\
\hline 399-8-5B & WHC & ER & $\overline{\mathrm{H}}$ & 34.1 & 34.09 & 34.08 \\
\hline $399-8-5 C$ & $\overline{\mathrm{WHC}}$ & $\overline{\mathrm{ER}}$ & $\overline{\mathrm{H}}$ & 46.55 & 46.54 & 46.53 \\
\hline 499-S0-7 & WHC & FFTF & $\bar{S}$ & 72.09 & 71.87 & 71.55 \\
\hline 499-S0-8 & WHC & FFTF & $\mathbf{S}$ & 37.67 & 37.44 & 37.11 \\
\hline 499-S1-7A & Orphan & & & 10.5 & 10.29 & 9.98 \\
\hline 499-S1-7B & WPPSS & WPPSS & $B$ & 34.85 & 34.65 & 34.34 \\
\hline 499-S1-7C & WPPSS? & ES\&M (ROM) & $S$ & 11.96 & 11.76 & 11.44 \\
\hline 499-S1-7E & Destroyed & Destroyed & & 9.68 & 9.48 & 9.17 \\
\hline $699-1-18$ & WHC & ES\&M (ROM) & $S$ & 7.14 & 6.74 & 6.31 \\
\hline 699-10-E12 & $\overline{\mathrm{PNL}}$ & Sitewide & $S$ & 80.39 & 80.41 & 80.41 \\
\hline 699-10-E12Q & PNL & Sitewide & & 7.24 & 7.26 & 7.25 \\
\hline $699-101-48 \mathrm{~A}$ & Orphan & ES\&M (ROM) & & 11.57 & 11.57 & 11.57 \\
\hline 699-101-48B & WHC & ES\&M (ROM) & $\mathbf{S}$ & 11.29 & 11.29 & 11.29 \\
\hline $699-101-48 \mathrm{C}$ & Orphan & ES\&M (ROM) & & 11.8 & 11.79 & 11.79 \\
\hline $699-11-45 A$ & PNL & Sitewide & $\mathbf{S}$ & 14.33 & 14.26 & 14.06 \\
\hline $699-11-45 \mathrm{C}$ & PNL & Sitewide & & 1.02 & 0.95 & 0.74 \\
\hline 699-14-38 & PNL & Sitewide & $S$ & 90.21 & 89.57 & 89.01 \\
\hline 699-14-38P & PNL & Sitewide & & 93.56 & 92.93 & 92.37 \\
\hline $699-14-38 Q$ & PNL & Sitewide & & 20.41 & 19.77 & 19.21 \\
\hline $699-14-47$ & $\overline{\mathrm{PNL}}$ & Sitewide & $\mathrm{B}$ & 1.96 & 1.82 & 1.53 \\
\hline 699-14-E6R & WHC & ES\&M (ROM) & & 89.48 & 89.51 & 89.49 \\
\hline 699-14-E6S & WHC & ES\&M (ROM) & & 61.25 & 61.28 & 61.25 \\
\hline 699-14-E6T & WHC & ES\&M (ROM) & $B$ & 8.03 & 8.06 & 8.03 \\
\hline $699-15-15 A$ & PNL & Sitewide & & 147.8 & 147.32 & 146.84 \\
\hline 699-15-15AP & PNL & Sitewide & & 19.72 & 19.25 & 18.77 \\
\hline 699-15-15B & PNL & Sitewide & $S$ & 2.96 & 2.48 & 2 \\
\hline $699-15-26$ & $\overline{\mathrm{PNL}}$ & Sitewide & $\mathbf{S}$ & 8 & 7.35 & 6.8 \\
\hline $699-17-26 A$ & WHC & ES\&M (ROM) & & $4 . \overline{36}$ & 3.69 & 3.14 \\
\hline 699-17-26BP & PNL-GLDR & PNL-GOLDER & & 51.89 & 51.23 & 50.68 \\
\hline 699-17-26BQ & $\overline{\text { PNL-GLDR }}$ & PNL-GOLDER & & 13.01 & 12.34 & 11.79 \\
\hline 699-17-26BR & PNL-GLDR & PNL-GOLDER & & 2.1 & 1.44 & 0.89 \\
\hline 699-17-26СР & PNL-GLDR & PNL-GOLDER & & 22.99 & 22.32 & 21.77 \\
\hline $699-17-26 \mathrm{CQ}$ & PNL-GLDR & PNL-GOLDER & & 13.55 & 12.88 & 12.33 \\
\hline 699-17-26CR & PNL-GLDR & PNL-GOLDER & & 2.9 & 2.24 & 1.68 \\
\hline $699-17-5$ & PNL & Sitewide & $\mathbf{S}$ & 1.49 & 1.43 & 1.21 \\
\hline
\end{tabular}




\begin{tabular}{|c|c|c|c|c|c|c|}
\hline 699-18-25A & \begin{tabular}{|l|} 
PNL \\
\end{tabular} & Sitewide & & 59.24 & 58.57 & 58.01 \\
\hline 699-18-25BR & PNL-GLDR & PNL-GOLDER & & 51.5 & 50.83 & 50.27 \\
\hline 699-18-28 & PNL-GLDR & PNL-GOLDER & & 5.06 & 4.38 & 3.82 \\
\hline 699-19-26A & $\overline{\text { PNL-GLDR }}$ & PNL-GOLDER & & 4.15 & 3.47 & 2.9 \\
\hline 699-19-26BP & PNL-GLDR & PNL-GOLDER & & 20.73 & 20.05 & 19.48 \\
\hline 699-19-26BQ & PNL-GLDR & PNL-GOLDER & & 3.02 & 2.34 & 1.77 \\
\hline $699-19-43$ & PNL & Sitewide & $S$ & 10.4 & 9.66 & 9.07 \\
\hline 699-19-58 & PNL & Sitewide & $S$ & 10.61 & 10.42 & 10.01 \\
\hline $699-2-3$ & $\overline{\mathrm{WHC}}$ & ES\&M (ROM) & $S$ & 8.14 & 7.98 & 7.71 \\
\hline 699-2-33A & PNL & Sitewide & $S$ & 12.77 & 12.55 & 12.22 \\
\hline $699-2-7$ & WHC & ES\&M (ROM) & S & 12.89 & 12.65 & 12.32 \\
\hline $699-20-20$ & PNL & Sitewide & $\bar{S}$ & 11.04 & 10.4 & 9.85 \\
\hline 699-20-39 & PNL & Sitewide & $S$ & 138.68 & 137.92 & 137.33 \\
\hline 699-20-39P & $\overline{\mathrm{PNL}}$ & Sitewide & & 23.39 & 22.63 & 22.04 \\
\hline 699-20-E12 & PNL & Sitewide & B & 20.9 & 20.91 & 20.91 \\
\hline 699-20-E12O & PNL & Sitewide & & 5.58 & 5.59 & 5.6 \\
\hline 699-20-E12Q & PNL & Sitewide & & 52.23 & 52.24 & 52.24 \\
\hline 699-20-E12R & PNL & Sitewide & & 35.47 & 35.48 & 35.48 \\
\hline 699-20-E12S & PNL & Sitewide & & 17.18 & 17.19 & 17.19 \\
\hline 699-20-E5A & PNL & \begin{tabular}{|l|} 
Sitewide \\
\end{tabular} & $S$ & 0.67 & 0.72 & 0.72 \\
\hline 699-20-E5R & WHC & $\overline{\mathrm{ES} \& \mathrm{M}(\mathrm{ROM})}$ & $\bar{B}$ & 63.73 & 63.78 & 63.78 \\
\hline 699-20-E5S & WHC & ES\&M (ROM) & & 36.71 & 36.77 & 36.76 \\
\hline 699-20-E5T & WHC & ES\&M (ROM) & & 6.8 & 6.86 & 6.85 \\
\hline $699-21-6$ & PNL & Sitewide & $S$ & 4.06 & 3.95 & 3.68 \\
\hline 699-23-34A & WHC & ES\&M (ROM) & $\mathrm{H}$ & 1.53 & 0.78 & 0.2 \\
\hline 699-24-1R & Orphan & ES\&M (ROM) & $\mathrm{B}$ & 65.67 & 65.71 & 65.64 \\
\hline 699-24-1S & Orphan & ES\&M (ROM) & $\bar{B}$ & 37.24 & 37.28 & 37.21 \\
\hline 699-24-1T & PNL & Sitewide & & 10.96 & 11 & 10.93 \\
\hline 699-24-33 & WHC & ES\&M (ROM) & S & 11.11 & 10.36 & 9.78 \\
\hline 699-24-34A & WHC & ES\&M (ROM) & $\mathrm{H}$ & 1.94 & 1.18 & 0.6 \\
\hline 699-24-34B & WHC & ES\&M (ROM) & $\mathrm{H}$ & 1.53 & 0.78 & 0.19 \\
\hline 699-24-34C & WHC & ES\&M (ROM) & $\overline{\mathrm{H}}$ & 1.63 & 0.88 & 0.29 \\
\hline 699-24-35 & WHC & ES\&M (ROM) & $\mathrm{H}$ & 1.83 & 1.07 & 0.48 \\
\hline 699-24-46 & PNL & Sitewide & $S$ & 12.51 & 11.72 & 11.12 \\
\hline 699-25-33A & WHC & ES\&M (ROM) & $S$ & 22.48 & 21.73 & 21.14 \\
\hline 699-25-33BP & PNL & Sitewide & & 28.57 & 27.81 & 27.23 \\
\hline 699-25-33BQ & PNL & Sitewide & & 20.3 & 19.54 & 18.95 \\
\hline $699-25-34 \mathrm{~A}$ & WHC & ES\&M (ROM) & $S$ & 3.03 & 2.27 & 1.68 \\
\hline 699-25-34B & WHC & ES\&M (ROM) & $S$ & 3.15 & 2.4 & 1.81 \\
\hline $699-25-34 \mathrm{C}$ & WHC & ES\&M (ROM) & $\mathrm{H}$ & 1.46 & 0.7 & 0.11 \\
\hline 699-25-34D & WHC & ES\&M (ROM) & $\mathrm{H}$ & 7.88 & 7.12 & 6.53 \\
\hline $699-25-55$ & PNL & Sitewide & $S$ & 11.62 & 10.84 & 10.2 \\
\hline $699-25-70$ & PNL & Sitewide & $S$ & 10.31 & 9.46 & 8.62 \\
\hline $699-26-15 \mathrm{~A}$ & PNL & Sitewide & $S$ & 13.63 & 13.12 & 12.62 \\
\hline 699-26-33 & WHC & ES\&M (ROM) & $S$ & 3.04 & 2.29 & 1.7 \\
\hline 699-26-34A & WHC & ES\&M (ROM) & $S$ & 3.24 & 2.48 & 1.89 \\
\hline
\end{tabular}




\begin{tabular}{|c|c|c|c|c|c|c|}
\hline $699-26-34 B$ & WHC & ES\&M (ROM) & $\bar{H}$ & 7.65 & 6.89 & 6.3 \\
\hline $699-26-35 A$ & WHC & ES\&M (ROM) & $S$ & 3.22 & 2.46 & 1.87 \\
\hline $699-26-35 \mathrm{C}$ & WHC & ES\&M (ROM) & $S$ & 21.99 & 21.23 & 20.64 \\
\hline 699-26-35DP & Orphan & ES\&M (ROM) & & 29.79 & 29.03 & 28.44 \\
\hline $699-26-35 \mathrm{DQ}$ & Orphan & ES\&M (ROM) & & 21.19 & 20.43 & 19.84 \\
\hline $699-26-89$ & PNL & Sitewide & $S$ & 15.19 & 14.86 & 14.49 \\
\hline $699-27-8$ & PNL & Sitewide & $S$ & 0.3 & 0.13 & -0.19 \\
\hline $699-28-40$ & PNL & Sitewide & $\bar{S}$ & 21.86 & 21.08 & 20.48 \\
\hline $699-28-40 \mathrm{P}$ & $\overline{\mathrm{PNL}}$ & Sitewide & $\bar{B}$ & 92.87 & 92.1 & 91.5 \\
\hline $699-28-40 Q$ & PNL & Sitewide & & 60.26 & 59.48 & 58.89 \\
\hline $699-28-52 A$ & PNL & Sitewide & $\mathrm{B}$ & 10.16 & 9.35 & 8.74 \\
\hline $699-28-55$ & Orphan & $\mathrm{ES} \& \mathrm{M}(\mathrm{ROM})$ & & -1.2 & -2.02 & -2.65 \\
\hline $699-29-4$ & PNL & Sitewide & $S$ & 1.05 & 1.04 & 0.91 \\
\hline $699-29-78$ & PNL & Sitewide & $\bar{S}$ & 23.12 & 22.09 & 21.22 \\
\hline $699-3-45$ & PNL & Sitewide & $\overline{\mathrm{B}}$ & 10.65 & 10.69 & 10.7 \\
\hline 699-31-11 & $\overline{\text { PNL }}$ & Sitewide & $S$ & 43.91 & 43.57 & 43.15 \\
\hline $699-31-31$ & PNL & Sitewide & $S$ & 46.43 & 45.68 & 45.09 \\
\hline 699-31-31P & PNL & Sitewide & & 143.97 & 143.22 & 142.63 \\
\hline $699-31-31 Q$ & PNL & Sitewide & & 73.86 & 73.11 & 72.52 \\
\hline $699-31-53 A$ & PNL & Sitewide & & 11.25 & 10.43 & 9.82 \\
\hline 699-31-53BO & $\overline{\mathrm{PNL}}$ & Sitewide & & 4.63 & 3.81 & 3.2 \\
\hline 699-31-53BP & PNL & Sitewide & & 38.16 & 37.34 & 36.73 \\
\hline $699-31-65$ & $\overline{\mathrm{WHC}}$ & ES\&M (ROM) & & 24.66 & 23.84 & 23.03 \\
\hline $699-31-650$ & WHC & ES\&M (ROM) & & 3.24 & 2.42 & 1.61 \\
\hline 699-31-65P & WHC & ES\&M (ROM) & & 55.06 & 54.24 & 53.43 \\
\hline 699-31-65Q & WHC & ES\&M (ROM) & & 42.87 & 42.05 & 41.24 \\
\hline 699-31-65R & WHC & ES\&M (ROM) & & 24.58 & 23.76 & 22.95 \\
\hline $699-32-42$ & Orphan & ES\&M (ROM) & & 1.5 & 0.71 & 0.11 \\
\hline 699-32-43 & PNL & Sitewide & $S$ & 1.74 & 0.95 & 0.35 \\
\hline $699-32-62$ & PNL & Sitewide & $\mathrm{B}$ & 14.37 & 13.54 & 12.84 \\
\hline 699-32-62P & PNL & Sitewide & & 63.45 & 62.61 & 61.91 \\
\hline $699-32-62 Q$ & PNL & Sitewide & & 25.04 & 24.21 & 23.51 \\
\hline 699-32-70B & PNL & Sitewide & $S$ & 33.58 & 32.61 & 31.73 \\
\hline $699-32-72$ & WHC & ES\&M (ROM) & & 81.56 & 80.52 & 79.64 \\
\hline 699-32-72P & WHC & ES\&M (ROM) & & 76.99 & 75.95 & 75.07 \\
\hline $699-32-77$ & PNL & Sitewide & $S$ & 29.01 & 27.92 & 27.05 \\
\hline 699-33-42 & PNL & Sitewide & $S$ & 1.63 & 0.84 & 0.24 \\
\hline $699-33-56$ & $\overline{\mathrm{PNL}}$ & Sitewide & $S$ & 29.04 & 28.22 & 27.6 \\
\hline $699-34-39 A$ & PNL & Sitewide & $S$ & 9.81 & 9.03 & 8.43 \\
\hline 699-34-41B & PNL & Sitewide & $\mathrm{B}$ & 1.97 & 1.18 & 0.58 \\
\hline 699-34-42 & PNL & Sitewide & $S$ & 2.18 & 1.39 & 0.79 \\
\hline $699-34-51$ & PNL & Sitewide & $S$ & 15.56 & 14.75 & 14.14 \\
\hline 699-34-88 & PNL & Sitewide & $\mathrm{B}$ & 14.44 & 13.95 & 13.45 \\
\hline 699-35-59 & US ECOL & US ECOL & $\mathrm{B}$ & 8.82 & 7.99 & 7.37 \\
\hline $699-35-66 \mathrm{~A}$ & PNL & Sitewide & $S$ & 8.75 & 7.89 & 7.09 \\
\hline $699-35-70$ & PNL & Sitewide & $\bar{B}$ & 1.7 & 0.7 & -0.17 \\
\hline
\end{tabular}




\begin{tabular}{|c|c|c|c|c|c|c|}
\hline $699-35-78 A$ & PNL & Sitewide & $S$ & 10.58 & 9.44 & 8.62 \\
\hline $699-35-9$ & PNL & Sitewide & $\overline{\mathrm{S}}$ & 1.2 & 1.01 & 0.72 \\
\hline $699-36-46 \mathrm{P}$ & Orphan & $\overline{\mathrm{ES} \& M}(\mathrm{ROM})$ & $\mathrm{B}$ & 66.19 & 65.39 & 64.78 \\
\hline $699-36-46 Q$ & PNL & Sitewide & & 45.1 & 44.29 & 43.69 \\
\hline $699-36-46 \mathrm{R}$ & Orphan & ES\&M (ROM) & $\bar{B}$ & 23.62 & 22.81 & 22.21 \\
\hline $699-36-46 S$ & WHC & ES\&M (ROM) & B & 2.53 & 1.72 & 1.12 \\
\hline $699-36-61 A$ & PNL & Sitewide & $\bar{S}$ & 5.97 & 5.13 & 4.51 \\
\hline 699-36-61B & PNL & Sitewide & $\mathrm{B}$ & 10.79 & 9.95 & 9.33 \\
\hline $699-36-93$ & PNL & Sitewide & $\mathrm{B}$ & 14.2 & 14.22 & 14.2 \\
\hline $699-37-43$ & PNL & Sitewide & $S$ & 14.13 & 13.33 & 12.73 \\
\hline $699-37-82 A$ & $\overline{\mathrm{PNL}}$ & Sitewide & $\mathbf{S}$ & 0.96 & 0.03 & -0.7 \\
\hline 699-37-82B & Orphan & ES\&M (ROM) & & 0.26 & -0.68 & -1.41 \\
\hline $699-37-82 \mathrm{BO}$ & Orphan & ES\&M (ROM) & & 4.15 & 3.21 & 2.49 \\
\hline 699-37-82BP & Orphan & ES\&M (ROM) & & 118.45 & 117.51 & 116.78 \\
\hline 699-37-82BQ & Orphan & ES\&M (ROM) & & 72.73 & 71.79 & 71.07 \\
\hline 699-37-82BR & Orphan & ES\&M (ROM) & & 48.35 & 47.41 & 46.68 \\
\hline 699-37-82BS & Orphan & ES\&M (ROM) & & 23.96 & 23.03 & 22.3 \\
\hline 699-37-E4 & PNL & Sitewide & $S$ & 21.57 & 21.58 & 21.57 \\
\hline $699-38-15$ & PNL & Sitewide & $S$ & 5.17 & 4.71 & 4.25 \\
\hline 699-38-65 & PNL & Sitewide & $S$ & 56.42 & 55.61 & 54.91 \\
\hline $699-38-65 P$ & PNL & Sitewide & & 53.37 & 52.56 & 51.86 \\
\hline $699-38-70$ & WHC & ES\&M (ROM) & $S$ & 35.82 & 34.79 & 33.93 \\
\hline 699-39-0 & PNL & Sitewide & $S$ & 2.79 & 2.8 & 2.77 \\
\hline 699-39-39 & PNL & Sitewide & $S$ & 18.45 & 17.68 & 17.08 \\
\hline 699-39-79 & WHC & ES\&M (ROM) & $S$ & 25.55 & 24.47 & 23.73 \\
\hline $699-40-1$ & PNL & Sitewide & $\bar{S}$ & 44.17 & 44.18 & 44.15 \\
\hline $699-40-33 A$ & $\mathrm{PNL}$ & Sitewide & $S$ & 15.19 & 14.37 & 13.76 \\
\hline $699-40-36$ & WHC & ES\&M (ROM) & $\overline{\mathrm{H}}$ & 28.61 & 27.84 & 27.25 \\
\hline 699-40-39 & WHC & ES\&M (ROM) & $\mathrm{H}$ & 22.34 & 21.56 & 20.97 \\
\hline $699-40-40 A$ & WHC & ES\&M (ROM) & $\mathrm{H}$ & 26.74 & 25.92 & 25.32 \\
\hline 699-40-40B & WHC & ES\&M (ROM) & $\mathrm{H}$ & 18.14 & 17.32 & 16.71 \\
\hline $699-40-62$ & PNL & Sitewide & $S$ & 5.84 & 5.01 & 4.4 \\
\hline 699-41-1 & PNL & Sitewide & $\bar{S}$ & 5.66 & 5.67 & 5.64 \\
\hline 699-41-23 & PNL & Sitewide & 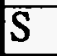 & 6.21 & 5.61 & 5.07 \\
\hline 699-41-35 & WHC & ES\&M (ROM) & $\mathrm{H}$ & 25.34 & 24.51 & 23.9 \\
\hline $699-41-40$ & WHC & ES\&M (ROM) & $\mathrm{H}$ & 9.66 & 8.8 & 8.2 \\
\hline $699-41-42$ & WHC & ES\&M (ROM) & $\mathrm{H}$ & 40.48 & 39.63 & 39.03 \\
\hline $699-42-12 A$ & PNL & Sitewide & $S$ & 11.97 & 11.8 & 11.58 \\
\hline $699-42-2$ & $\mathrm{PNL}$ & Sitewide & $S$ & 5.93 & 5.93 & 5.89 \\
\hline $699-42-37$ & WHC & ES\&M (ROM) & $\overline{\mathrm{H}}$ & 11.99 & 11.14 & 10.54 \\
\hline $699-42-39 A$ & WHC & ES\&M (ROM) & $\mathrm{H}$ & 7.84 & 6.98 & 6.37 \\
\hline 699-42-39B & WHC & ES\&M (ROM) & $\mathrm{H}$ & 18.06 & 17.2 & 16.59 \\
\hline $699-42-40 \mathrm{~A}$ & WHC & ES\&M (ROM) & $S$ & 8.86 & 7.98 & 7.37 \\
\hline $699-42-40 \mathrm{~B}$ & PNL & Sitewide & $\mathrm{B}$ & 2.18 & 1.29 & 0.68 \\
\hline $699-42-41$ & WHC & ES\&M (ROM) & $\mathrm{H}$ & -2.67 & -3.54 & -4.15 \\
\hline $699-42-42 B$ & WHC & ES\&M (ROM) & $\mathrm{H}$ & 6.89 & 6.03 & 5.42 \\
\hline
\end{tabular}




\begin{tabular}{|c|c|c|c|c|c|c|}
\hline $699-43-104$ & PNL & Sitewide & $\mathrm{B}$ & 23.12 & 23.19 & 23.22 \\
\hline $699-43-3$ & PNL & Sitewide & $S$ & 9.24 & 9.23 & 9.19 \\
\hline $699-43-40$ & WHC & ES\&M (ROM) & $\overline{\mathrm{H}}$ & -0.96 & -1.87 & -2.48 \\
\hline $699-43-41 E$ & WHC & ES\&M (ROM) & $\mathrm{H}$ & -0.44 & -1.33 & -1.95 \\
\hline $699-43-41 F$ & WHC & ES\&M (ROM) & $\mathrm{H}$ & 8.61 & 7.71 & 7.1 \\
\hline $699-43-41 G$ & WHC & ES\&M (ROM) & $\mathrm{H}$ & 15.49 & 14.6 & 13.99 \\
\hline $699-43-42$ & WHC & ES\&M (ROM) & $\bar{B}$ & 17.96 & 17.08 & 16.47 \\
\hline $699-43-42 \mathrm{~J}$ & WHC & ES\&M (ROM) & $\mathrm{H}$ & -0.36 & -1.23 & -1.84 \\
\hline $699-43-43$ & WHC & ES\&M (ROM) & $\mathrm{H}$ & 0.34 & -0.51 & -1.12 \\
\hline 699-43-45 & WHC & ES\&M (ROM) & $\mathrm{H}$ & 2.62 & 1.78 & 1.17 \\
\hline $699-43-88$ & WHC & ER & $\mathbf{S}$ & 3.77 & 3.51 & 3.23 \\
\hline $699-43-89$ & PNL & Sitewide & B & 21.05 & 20.8 & 20.52 \\
\hline 699-44-39B & WHC & ES\&M (ROM) & $\overline{\mathrm{H}}$ & 3.04 & 2.11 & 1.5 \\
\hline $699-44-4$ & $\mathrm{PNL}$ & Sitewide & $\overline{\mathrm{B}}$ & 7.54 & 7.53 & 7.5 \\
\hline $699-44-42$ & WHC & ES\&M (ROM) & $\mathrm{H}$ & -1.28 & -2.18 & -2.79 \\
\hline $699-44-43 B$ & WHC & ES\&M (ROM) & $\mathrm{H}$ & -0.25 & -1.11 & -1.72 \\
\hline $699-44-64$ & $\overline{\mathrm{PNL}}$ & Sitewide & $S$ & 37.06 & 36.24 & 35.63 \\
\hline $699-45-2$ & PNL & Sitewide & $S$ & 5.6 & 5.59 & 5.58 \\
\hline $699-45-42$ & PNL & Sitewide & $S$ & 1.89 & 0.98 & 0.37 \\
\hline $699-45-69 A$ & WHC & ES\&M (ROM) & $S$ & 24.47 & 24.05 & 23.61 \\
\hline 699-46-21B & PNL & Sitewide & $S$ & 2.41 & 2.21 & 1.95 \\
\hline $699-46-4$ & $\overline{\text { PNL-GLDR }}$ & PNL-GOLDER & & 7.67 & 7.66 & 7.63 \\
\hline $699-47-35 A$ & PNL & Sitewide & $S$ & 9.25 & 8.42 & 7.84 \\
\hline $699-47-35 B$ & WHC & ES\&M (ROM) & $\mathrm{B}$ & 7.62 & 6.81 & 6.22 \\
\hline $699-47-35 \mathrm{C}$ & Orphan & ES\&M (ROM) & & 8.62 & 7.8 & 7.21 \\
\hline $699-47-5$ & PNL & Sitewide & $S$ & 6.57 & 6.57 & 6.55 \\
\hline $699-47-60$ & PNL & Sitewide & $S$ & 8.62 & 7.79 & 7.2 \\
\hline 699-48-18 & PNL & Sitewide & $S$ & 5.52 & 5.43 & 5.32 \\
\hline $699-48-50$ & WHC & ES\&M (ROM) & $\mathrm{H}$ & 2.54 & 1.71 & 1.11 \\
\hline $699-48-71$ & PNL & Sitewide & $S$ & 16.36 & 16.26 & 16.12 \\
\hline $699-48-77 A$ & WHC & ES\&M (ROM) & $\overline{\mathrm{H}}$ & 3.87 & 4.17 & 3.96 \\
\hline $699-49-12 A$ & Orphan & ES\&M (ROM) & & 12.14 & 12.09 & 12.04 \\
\hline $699-49-12 \mathrm{~B}$ & Orphan & ES\&M (ROM) & & 11.1 & 11.06 & 11.01 \\
\hline $699-49-13 \mathrm{C}$ & Orphan & ES\&M (ROM) & & 3.05 & 3 & 2.95 \\
\hline 699-49-13D & Orphan & ES\&M (ROM) & & 9.25 & 9.2 & 9.15 \\
\hline 699-49-13E & PNL & Sitewide & $S$ & 7.64 & 7.59 & 7.54 \\
\hline 699-49-28 & PNL & Sitewide & $\mathrm{B}$ & -0.39 & -0.19 & -0.13 \\
\hline $699-49-55 A$ & WHC & ES\&M (ROM) & $\overline{\mathrm{H}}$ & 1.98 & 1.15 & 0.56 \\
\hline 699-49-57A & WHC & ES\&M (ROM) & & 3.05 & 2.23 & 1.63 \\
\hline $699-49-79$ & WHC & ES\&M (ROM) & $S$ & 9.11 & 9.01 & 8.81 \\
\hline $699-50-28 B$ & WHC & ES\&M (ROM) & S & 1.62 & 1.82 & 1.93 \\
\hline $699-50-30$ & PNL & Sitewide & $\bar{B}$ & 25.89 & 26.08 & 26.15 \\
\hline $699-50-42$ & PNL & Sitewide & $s$ & .0 .49 & -0.51 & -1.15 \\
\hline $699-50-42 P$ & PNL & Sitewide & & 16.03 & 15.03 & 14.39 \\
\hline $699-50-53 A$ & WHC & ES\&M (ROM) & $\mathrm{H}$ & 0.46 & -0.37 & -0.96 \\
\hline 699-50-53B & WHC & ES\&M (ROM) & $\overline{\mathrm{H}}$ & 21.19 & 20.37 & 19.77 \\
\hline
\end{tabular}




\begin{tabular}{|c|c|c|c|c|c|c|}
\hline 699-50-85 & PNL & Sitewide & $S$ & 22.73 & 22.58 & 22.45 \\
\hline $699-50-85 P$ & $\overline{\mathrm{PNL}}$ & Sitewide & & 73.02 & 72.88 & 72.74 \\
\hline $699-51-36 \mathrm{~B}$ & $\overline{\mathrm{PNL}}$ & Sitewide & & 14.39 & 14.14 & 13.77 \\
\hline $699-51-36 C$ & $\overline{\mathrm{PNL}}$ & Sitewide & & 13.78 & 13.53 & 13.15 \\
\hline $699-51-63$ & $\overline{\text { PNL }}$ & Sitewide & $\bar{S}$ & 4.27 & 3.46 & 2.87 \\
\hline $699-51-75$ & $\overline{\mathrm{PNL}}$ & Sitewide & $S$ & 52.78 & 53.16 & 53.27 \\
\hline $699-51-75 P$ & PNL & Sitewide & & 54.3 & 54.68 & 54.79 \\
\hline $699-52-18 C$ & Orphan & ES\&M (ROM) & & 16.05 & 16.04 & 16.01 \\
\hline $699-52-19$ & PNL & Sitewide & $\bar{B}$ & 12.69 & 12.68 & 12.65 \\
\hline $699-52-54$ & WHC & ES\&M (ROM) & $\bar{H}$ & 0.23 & -0.58 & -1.17 \\
\hline $699-52-57$ & WHC & $\mathrm{ES} \& \mathrm{M}(\mathrm{ROM})$ & $\overline{\mathrm{H}}$ & 0.01 & -0.81 & -1.4 \\
\hline $699-53-47 A$ & PNL & Sitewide & $S$ & -0.86 & -1.69 & -2.29 \\
\hline $699-53-47 B$ & WHC & ES\&M (ROM) & $\bar{S}$ & 3.01 & 2.18 & 1.58 \\
\hline $699-53-48 A$ & PNL & Sitewide & $\bar{S}$ & 2.56 & 1.72 & 1.13 \\
\hline $699-53-48 B$ & WHC & $\overline{\mathrm{ES}} \& \mathrm{M}(\mathrm{ROM})$ & $\bar{S}$ & -1 & -1.83 & -2.42 \\
\hline $699-53-55 \AA$ & WHC & ES\&M (ROM) & $\overline{\mathrm{H}}$ & 32.23 & 31.41 & 30.83 \\
\hline $699-53-55 B$ & WHC & ES\&M (ROM) & $\overline{\mathrm{H}}$ & 23.61 & 22.8 & 22.21 \\
\hline 699-53-55C & WHC & ES\&M (ROM) & $\mathrm{H}$ & 14.23 & 13.41 & 12.82 \\
\hline $699-54-18 \mathrm{~A}$ & Orphan & ES\&M (ROM) & & 9.96 & 9.95 & 9.93 \\
\hline $699-54-18 C$ & Orphan & ES\&M (ROM) & & 19.11 & 19.1 & 19.09 \\
\hline $699-54-42$ & PNL & Sitewide & $\bar{B}$ & 27.9 & 27.05 & 26.44 \\
\hline $699-54-45 A$ & $\overline{\mathrm{PNL}}$ & Sitewide & $\bar{B}$ & 4.06 & 3.21 & 2.61 \\
\hline $699-54-48$ & $\overline{\mathrm{PNL}}$ & Sitewide & $\overline{\mathrm{S}}$ & 2.56 & $1 . \overline{73}$ & 1.14 \\
\hline $699-55-40$ & PNL & Sitewide & $\overline{\mathrm{B}}$ & 0.19 & $-0 . \overline{38}$ & -0.93 \\
\hline $699-55-44$ & PNL & Sitewide & B & 10.04 & 9.2 & 8.6 \\
\hline 699-55-50A & $\mathrm{PNL}$ & Sitewide & $\overline{\mathrm{B}}$ & 18.14 & 17.32 & 16.73 \\
\hline $699-55-50 \mathrm{AO}$ & PNL & Sitewide & & 2.83 & 2.01 & 1.42 \\
\hline 699-55-50AP & $\overline{\mathrm{PNL}}$ & Sitewide & & 17.59 & 16.77 & 16.18 \\
\hline 699-55-50AQ & PNL & Sitewide & & 11.52 & 10.7 & 10.11 \\
\hline $699-55-50 \mathrm{C}$ & WHC & ES\&M (ROM) & $S$ & 5.15 & 4.33 & 3.74 \\
\hline 699-55-50D & PNL & Sitewide & $\overline{\mathrm{B}}$ & 15.82 & 15 & 14.41 \\
\hline $699-55-55$ & WHC & ES\&M (ROM) & $\overline{\mathrm{H}}$ & 2.42 & 1.61 & 1.03 \\
\hline $699-55-57$ & WHC & ES\&M (ROM) & $\mathrm{H}$ & 0.89 & 0.08 & -0.5 \\
\hline $699-55-60 A$ & Orphan & ES\&M (ROM) & & 17.97 & 17.18 & 16.61 \\
\hline 699-55-60B & Orphan & ES\&M (ROM) & $\bar{B}$ & 34.48 & 33.69 & 33.13 \\
\hline $699-55-70$ & PNL & Sitewide & B & 19.12 & 19.38 & 19.53 \\
\hline 699-55-70P & PNL & Sitewide & & 18.66 & 18.92 & 19.08 \\
\hline $699-55-76$ & PNL & Sitewide & $\mathbf{S}$ & 22.98 & 23.17 & 23.33 \\
\hline $699-55-89$ & PNL & Sitewide & $S$ & 15.38 & 15.6 & 15.74 \\
\hline $699-55-95$ & $\overline{\mathrm{PNL}}$ & Sitewide & B & 22.38 & 22.55 & 22.65 \\
\hline $699-55-95 \mathrm{P}$ & $\overline{\mathrm{PNL}}$ & Sitewide & & 45.14 & 45.32 & 45.42 \\
\hline $699-55-95 Q$ & PNL & Sitewide & & 44.84 & 45.02 & 45.12 \\
\hline $699-56-26 B$ & Orphan & ES\&M (ROM) & & 8.54 & 8.51 & 8.47 \\
\hline $699-57-25 \mathrm{~A}$ & $\mathrm{PNL}$ & Sitewide & $S$ & 9.16 & 9.13 & 9.09 \\
\hline $699-57-29 A$ & PNL & Sitewide & $\bar{S}$ & 6.87 & 6.84 & 6.79 \\
\hline $699-57-29 B$ & PNL & Sitewide & B & 9.1 & 9.06 & 9.01 \\
\hline
\end{tabular}




\begin{tabular}{|c|c|c|c|c|c|c|}
\hline $699-57-59$ & WHC & ES\&M (ROM) & $\mathrm{H}$ & 3.46 & 2.71 & 2.15 \\
\hline $699-57-83 A$ & $\overline{\mathrm{PNL}}$ & Sitewide & $\mathbf{S}$ & 14.44 & 14.59 & 14.7 \\
\hline $699-58-24$ & PNL & Sitewide & B & 1.7 & 1.68 & 1.65 \\
\hline 699-59-33 & Orphan & Orphan & & 2.3 & 2.24 & 2.15 \\
\hline $699-59-58$ & PNL & Sitewide & $S$ & 3.94 & 3.22 & 2.69 \\
\hline 699-59-80B & PNL & Sitewide & $\bar{B}$ & 9.46 & 9.62 & 9.76 \\
\hline 699-60-57 & PNL & Sitewide & $S$ & 1.66 & 0.98 & 0.47 \\
\hline 699-60-60. & WHC & ES\&M (ROM) & $\bar{B}$ & -0.55 & -1.24 & -1.75 \\
\hline 699-60-60P & WHC & ES\&M (ROM) & & 4.02 & 3.33 & 2.82 \\
\hline $699-61-37$ & PNL & Sitewide & $\bar{S}$ & 2.23 & 2.07 & 1.88 \\
\hline $699-61-41$ & $\overline{\mathrm{PNL}}$ & Sitewide & $S$ & -1.2 & -1.46 & -1.75 \\
\hline $699-61-62$ & $\overline{\mathrm{PNL}}$ & Sitewide & $S$ & 0.67 & 0.02 & -0.47 \\
\hline 699-61-66 & PNL & Sitewide & $S$ & 11.2 & 10.61 & 10.17 \\
\hline $699-62-31$ & PNL & Sitewide & $\bar{S}$ & 5.49 & 5.43 & 5.34 \\
\hline $699-62-43 A$ & PNL & Sitewide & $\bar{B}$ & 6.51 & 6.19 & 5.85 \\
\hline $699-62-43 C$ & Orphan & ES\&M (ROM) & & 7.84 & 7.52 & 7.19 \\
\hline 699-62-43D & Orphan & ES\&M (ROM) & & 7.84 & 7.52 & 7.19 \\
\hline $699-62-43 E$ & Orphan & ES\&M (ROM) & & 12.65 & 12.33 & 12 \\
\hline $699-62-43 \mathrm{~F}$ & PNL & Sitewide & $S$ & 11.62 & 11.3 & 10.96 \\
\hline $699-62-43 G$ & Orphan & ES\&M (ROM) & & 12.67 & 12.35 & 12.01 \\
\hline $699-62-43 \mathrm{H}$ & Orphan & ES\&M (ROM) & & 12.53 & 12.21 & 11.87 \\
\hline 699-62-43I & Orphan & ES\&M (ROM) & & 10.55 & 10.23 & 9.9 \\
\hline $699-62-43 J$ & Orphan & ES\&M (ROM) & & 10.84 & 10.51 & 10.17 \\
\hline $699-62-43 K$ & Orphan & ES\&M (ROM) & & 10.06 & 9.74 & 9.41 \\
\hline $699-62-43 \mathrm{~L}$ & Orphan & ES\&M (ROM) & & 13.14 & 12.81 & 12.48 \\
\hline $699-62-43 \mathrm{M}$ & Orphan & ES\&M (ROM) & & 10.38 & 10.06 & 9.72 \\
\hline $699-62-43 N$ & Orphan & ES\&M (ROM) & & 6.25 & 5.93 & 5.59 \\
\hline $699-63-25 A$ & PNL & Sitewide & $S$ & 20 & 19.99 & 19.96 \\
\hline $699-63-51$ & PNL & Sitewide & $S$ & 0.81 & 0.32 & -0.1 \\
\hline 699-63-55 & PNL & Sitewide & $\mathbf{S}$ & 11.03 & 10.47 & 10.03 \\
\hline 699-63-58 & PNL & Sitewide & $S$ & 8.55 & 7.93 & 7.46 \\
\hline 699-63-90 & $\mathrm{PNL}$ & Sitewide & $S$ & 11.87 & 11.89 & 11.89 \\
\hline 699-64-27 & PNL & Sitewide & $S$ & 7.99 & 7.95 & 7.89 \\
\hline 699-64-62 & PNL & Sitewide & $\bar{S}$ & 2.76 & 2.16 & 1.7 \\
\hline $699-65-50$ & PNL & Sitewide & $S$ & 16.44 & 15.96 & 15.54 \\
\hline $699-65-59 A$ & PNL & Sitewide & $\mathbf{S}$ & 5.01 & 4.43 & 3.98 \\
\hline 699-65-59B & PNL & Sitewide & & 25.1 & 24.52 & 24.08 \\
\hline 699-65-59C & PNL & Sitewide & & 9.86 & 9.28 & 8.84 \\
\hline $699-65-72$ & PNL & Sitewide & $S$ & 3.95 & 3.66 & 3.44 \\
\hline 699-66-23 & PNL & Sitewide & $S$ & 21.83 & 21.82 & 21.81 \\
\hline 699-66-38 & $\mathrm{PNL}$ & Sitewide & $S$ & 27.02 & 26.85 & 26.63 \\
\hline 699-66-39 & PNL & Sitewide & $S$ & -1.78 & -1.99 & -2.24 \\
\hline 699-66-58 & PNL & Sitewide & $S$ & 2.18 & 1.62 & 1.19 \\
\hline 699-66-64 & $\mathrm{PNL}$ & Sitewide & $S$ & 2.5 & 1.98 & 1.56 \\
\hline 699-67-51P & PNL & Sitewide & & 32.98 & 32.49 & 32.08 \\
\hline $699-67-51 Q$ & PNL & Sitewide & & 20.03 & 19.54 & 19.13 \\
\hline
\end{tabular}




\begin{tabular}{|c|c|c|c|c|c|c|}
\hline 699-67-86 & PNL & Sitewide & $S$ & 117.23 & 117.21 & 117.2 \\
\hline $699-67-98$ & $\overline{\mathrm{PNL}}$ & Sitewide & $S$ & 22.11 & 22.11 & 22.11 \\
\hline $699-68-105$ & $\overline{\text { PNL }}$ & Sitewide & $\bar{B}$ & 10.94 & 10.94 & 10.94 \\
\hline $699-69-45$ & PNL & Sitewide & & 62.38 & 62.03 & 61.68 \\
\hline $699-69-450$ & PNL & Sitewide & & 6.53 & 6.17 & 5.83 \\
\hline $699-69-45 P$ & PNL & Sitewide & & 55.3 & 54.94 & 54.6 \\
\hline 699-69-45Q & PNL & Sitewide & & 62.31 & 61.96 & 61.61 \\
\hline 699-69-45R & PNL & Sitewide & & 25.04 & 24.68 & 24.33 \\
\hline $699-70-68$ & WHC & ER & $\mathbf{S}$ & 5.07 & 4.73 & 4.46 \\
\hline $699-71-30$ & PNL & Sitewide & $\mathbf{S}$ & 16.52 & 16.49 & 16.44 \\
\hline $699-71-52$ & PNL & Sitewide & $\bar{S}$ & 10.07 & 9.61 & 9.22 \\
\hline 699-71-77 & PNL & Sitewide & $\bar{S}$ & 63.81 & 63.75 & 63.7 \\
\hline $699-72-73$ & $\overline{\mathrm{PNL}}$ & Sitewide & $S$ & 26.51 & 26.4 & 26.31 \\
\hline $699-72-88$ & PNL & Sitewide & $\bar{S}$ & 1.99 & 1.98 & 1.98 \\
\hline $699-72-92$ & WHC & ER & $S$ & 42.72 & 42.72 & 42.72 \\
\hline 699-73-61 & PNL & Sitewide & $S$ & 3.3 & 2.85 & 2.48 \\
\hline $699-74-44$ & $\overline{\text { PNL }}$ & Sitewide & $S$ & 3.69 & 3.43 & 3.15 \\
\hline $699-74-48$ & $\overline{\text { PNL }}$ & Sitewide & $\bar{B}$ & 16.23 & 15.86 & 15.52 \\
\hline $699-77-36$ & PNL & Sitewide & S & 14.17 & 14.12 & 14.04 \\
\hline $699-77-54$ & PNL & Sitewide & $S$ & 10.38 & 10 & 9.66 \\
\hline $699-78-62$ & PNL & Sitewide & $\bar{S}$ & 9.63 & 9.29 & 9.01 \\
\hline $699-8-17$ & PNL & Sitewide & $S$ & 9.84 & 9.36 & 8.89 \\
\hline $699-8-25$ & PNL & Sitewide & $S$ & 15.53 & 14.98 & 14.48 \\
\hline $699-8-32$ & PNL & Sitewide & $\bar{S}$ & $1: 87$ & 1.37 & 0.88 \\
\hline 699-80-43P & Orphan & ES\&M (ROM) & $\bar{B}$ & 127.37 & 127.23 & 127.04 \\
\hline $699-80-43 Q$ & Orphan & ES\&M (ROM) & $B$ & 62.26 & 62.11 & 61.92 \\
\hline $699-80-43 R$ & Orphan & ES\&M (ROM) & $\overline{\mathrm{B}}$ & 34.64 & 34.49 & 34.3 \\
\hline $699-80-43 S$ & WHC & ES\&M (ROM) & $\bar{B}$ & 7.27 & 7.13 & 6.94 \\
\hline $699-81-58$ & WHC & ES\&M (ROM) & $S$ & 30.77 & 30.5 & 30.27 \\
\hline 699-83-47 & PNL & Sitewide & $S$ & 31.02 & 30.88 & 30.71 \\
\hline 699-84-35AQ & $\overline{\mathrm{PNL}}$ & Sitewide & & 77.84 & 77.83 & 77.81 \\
\hline 699-84-35AR & $\overline{\mathrm{PNL}}$ & Sitewide & & 58.34 & 58.32 & 58.3 \\
\hline 699-84-35AS & PNL & Sitewide & & 38.83 & 38.82 & 38.79 \\
\hline $699-87-55$ & PNL & Sitewide & $\mathbf{S}$ & 6.51 & 6.4 & 6.29 \\
\hline $699-89-35$ & PNL & Sitewide & $S$ & 15.19 & 15.19 & 15.19 \\
\hline 699-9-E2 & PNL & Sitewide & $S$ & 4.7 & 4.7 & 4.58 \\
\hline $699-90-45$ & $\overline{\text { PNL }}$ & Sitewide & $\bar{S}$ & 0.4 & 0.37 & 0.31 \\
\hline 699-93-48A & PNL & Sitewide & $\overline{\mathrm{H}}$ & 1.95 & 1.92 & 1.88 \\
\hline $699-96-43$ & PNL & Sitewide & $\overline{\mathrm{H}}$ & 1.58 & 1.57 & 1.56 \\
\hline $699-96-49$ & $\overline{\text { PNL }}$ & Sitewide & $S$ & 17.66 & 17.65 & 17.64 \\
\hline 699-96-49P & PNL & Sitewide & & 15.52 & 15.51 & 15.5 \\
\hline $699-97-43$ & PNL & Sitewide & $S$ & 16.39 & 16.39 & 16.38 \\
\hline 699-97-51A & PNL & Sitewide & $S$ & 5.63 & 5.63 & 5.62 \\
\hline 699-S12-3 & PNL & Sitewide & $\overline{\mathrm{B}}$ & 12.46 & 12.46 & 12.38 \\
\hline 699-S14-20A & PNL & Sitewide & $S$ & 7.05 & 6.99 & 6.82 \\
\hline 699-S18-E2A & PNL & Sitewide & $\mathrm{B}$ & 48.8 & 48.79 & 48.76 \\
\hline
\end{tabular}




\begin{tabular}{|c|c|c|c|c|c|c|}
\hline 699-S18-E2AP & PNL & Sitewide & & 54.82 & 54.8 & 54.77 \\
\hline 699-S19-11 & PNL & Sitewide & $\mathbf{S}$ & 2.17 & 2.15 & 2.04 \\
\hline 699-S19-E13 & PNL & Sitewide & $S$ & 9.09 & 9.08 & 9.08 \\
\hline 699-S19-E14 & $\overline{\mathrm{PNL}}$ & Sitewide & $\overline{\mathrm{H}}$ & 3.63 & 3.63 & 3.63 \\
\hline 699-S22-E9A & $\overline{\mathrm{PNL}}$ & Sitewide & $\overline{\mathrm{H}}$ & 3.44 & 3.43 & 3.42 \\
\hline 699-S22-E9B & PNL & Sitewide & $\mathrm{H}$ & 37.24 & 37.22 & 37.21 \\
\hline 699-S22-E9C & PNL & Sitewide & & 47.07 & 47.06 & 47.05 \\
\hline 699-S27-E14 & $\overline{\mathrm{PNL}}$ & Sitewide & $S$ & 15.58 & 15.56 & 15.56 \\
\hline 699-S27-E9A & PNL & Sitewide & $\mathrm{H}$ & 4.01 & 3.96 & 3.94 \\
\hline 699-S27-E9B & PNL & Sitewide & $\overline{\mathrm{H}}$ & 40.72 & 40.67 & 40.65 \\
\hline 699-S27-E9C & $\overline{\mathrm{PNL}}$ & Sitewide & $\overline{\mathrm{H}}$ & 48.13 & 48.08 & 48.06 \\
\hline 699-S28-E0 & PNL & Sitewide & $S$ & 26.35 & 26.27 & 26.22 \\
\hline 699-S28-E12 & $\overline{\mathrm{PNL}}$ & Sitewide & $\overline{\mathrm{H}}$ & 4.74 & 4.7 & 4.69 \\
\hline 699-S29-E11 & USACE & ER & & 2.19 & 2.12 & 2.11 \\
\hline 699-S29-E12 & USACE & ER & $S$ & 11.56 & 11.51 & 11.5 \\
\hline 699-S29-E16A & PNL & Sitewide & $\mathrm{H}$ & 4 & 4 & 4 \\
\hline 699-S29-E16B & PNL & Sitewide & $\mathrm{H}$ & 21.03 & 21.02 & 21.02 \\
\hline 699-S29-E16C & $\overline{\mathrm{PNL}}$ & Sitewide & & 43.1 & 43.09 & 43.09 \\
\hline 699-S3-25 & PNL & Sitewide & $S$ & 12.28 & 12.06 & 11.75 \\
\hline 699-S3-E12 & PNL & Sitewide & $\mathbf{S}$ & 11.1 & 11.11 & 11.1 \\
\hline 699-S30-E10A & USACE & ER & $\overline{\mathrm{H}}$ & 3.48 & 3.4 & 3.38 \\
\hline 699-S30-E10B & USACE & ER & $\overline{\mathrm{H}}$ & 3.5 & 3.41 & 3.39 \\
\hline 699-S30-E15A & PNL & Sitewide & $\overline{\mathrm{H}}$ & 7.08 & 7.07 & 7.06 \\
\hline 699-S31-1 & PNL & Sitewide & $S$ & -0.42 & -0.53 & -0.58 \\
\hline 699-S31-E10A & USACE & ER & $\overline{\mathrm{H}}$ & 3.37 & 3.27 & 3.25 \\
\hline 699-S31-E10B & USACE & ER & $\overline{\mathrm{H}}$ & 2.03 & 1.93 & 1.91 \\
\hline 699-S31-E10C & USACE & ER & $\overline{\mathrm{H}}$ & 5.96 & 5.86 & 5.84 \\
\hline 699-S31-E10D & USACE & ER & $\mathrm{H}$ & 2.44 & 2.34 & 2.32 \\
\hline 699-S31-E10E & PNL & Sitewide & $\overline{\mathrm{H}}$ & 19.5 & 19.4 & 19.38 \\
\hline 699-S31-E11 & USACE & ER & & 3.3 & 3.21 & 3.2 \\
\hline 699-S32-E11 & USACE & ER & & 4 & 3.87 & 3.85 \\
\hline 699-S32-E13A & WHC & ER & $S$ & 8.09 & 8.02 & 8.01 \\
\hline 699-S32-E13B & WHC & ER & $S$ & 6.94 & 6.86 & 6.85 \\
\hline 699-S32-E8 & PNL & Sitewide & $\mathrm{H}$ & 16.59 & 16.46 & 16.43 \\
\hline 699-S34-E10 & USACE & ER & $\overline{\mathrm{H}}$ & 3.02 & 2.84 & 2.82 \\
\hline 699-S34-E15 & PNL & Sitewide & & 0.81 & 0.73 & 0.72 \\
\hline 699-S36-E12B & USACE & ER & $B$ & 4.89 & 4.72 & 4.7 \\
\hline 699-S36-E13A & WHC & ER & $S$ & 6.74 & 6.59 & 6.58 \\
\hline 699-S36-E13B & USACE & $\overline{\mathrm{ER}}$ & $\bar{B}$ & 4.6 & 4.44 & 4.42 \\
\hline 699-S37-E11A & USACE & ER & $\overline{\mathrm{H}}$ & 2.7 & 2.48 & 2.46 \\
\hline 699-S37-E14 & PNL & Sitewide & $\mathrm{H}$ & 1.02 & 0.89 & 0.87 \\
\hline 699-S38-E11 & PNL & Sitewide & $\mathrm{H}$ & 3.13 & 2.87 & 2.84 \\
\hline 699-S38-E12A & USACE & ER & $\overline{\mathrm{H}}$ & 3.59 & 3.38 & 3.36 \\
\hline 699-S38-E12B & PNL & Sitewide & $\bar{H}$ & 10.56 & 10.35 & 10.32 \\
\hline 699-S40-E13A & USACE & ER & $\overline{\mathrm{H}}$ & 3.78 & 3.57 & 3.55 \\
\hline 699-S40-E13B & USACE & ER & $\mathrm{H}$ & 3.24 & 3.04 & 3.01 \\
\hline
\end{tabular}




\begin{tabular}{|c|c|c|c|c|c|c|}
\hline 699-S40-E13C & USACE & ER & $\mathrm{H}$ & 3.53 & 3.33 & 3.3 \\
\hline 699-S40-E14 & WHC & $\overline{\mathrm{ER}}$ & $\mathrm{H}$ & 2.94 & 2.77 & 2.75 \\
\hline 699-S41-E11A & USACE & ER & $\mathrm{H}$ & 2.59 & 2.29 & 2.26 \\
\hline 699-S41-E12 & USACE & $\overline{\mathrm{ER}}$ & $\mathrm{H}$ & 4.59 & 4.32 & 4.29 \\
\hline 699-S41-E13A & PNL & Sitewide & $\mathrm{H}$ & -0.19 & -0.42 & -0.44 \\
\hline 699-S41-E13B & PNL & Sitewide & $\overline{\mathrm{H}}$ & 8.91 & 8.68 & 8.65 \\
\hline 699-S41-E13C & $\overline{\mathrm{PNL}}$ & Sitewide & $\mathrm{H}$ & 19.25 & 19.02 & 18.99 \\
\hline 699-S43-E12 & $\overline{\text { PNL }}$ & Sitewide & $\overline{\mathrm{H}}$ & 0.84 & 0.57 & 0.54 \\
\hline 699-S6-E14A & PNL & Sitewide & $\bar{S}$ & 10.75 & 10.75 & 10.75 \\
\hline 699-S6-E4B & PNL & Sitewide & $S$ & 5.63 & 5.64 & 5.58 \\
\hline 699-S6-E4C & $\overline{\mathrm{PNL}}$ & Sitewide & & 58.01 & 58.02 & 57.96 \\
\hline 699-S6-E4D & PNL & Sitewide & $S$ & 1.71 & 1.72 & 1.66 \\
\hline 699-S6-E4E & PNL & Sitewide & & 11.27 & 11.28 & 11.22 \\
\hline 699-S6-E4F & PNL & Sitewide & & 13.16 & 13.17 & 13.11 \\
\hline 699-S6-E4G & PNL & Sitewide & & 11.53 & 11.54 & 11.49 \\
\hline 699-S7-34 & PNL & Sitewide & $\mathrm{B}$ & 2.46 & 2.49 & 2.42 \\
\hline 699-S7-34Q & PNL & Sitewide & & 39.64 & 39.67 & 39.61 \\
\hline 699-S8-19 & PNL & Sitewide & $S$ & \begin{tabular}{|l|}
4.37 \\
\end{tabular} & 4.28 & 4.06 \\
\hline
\end{tabular}


Appendix D

Hydrographs of Selected Monitoring Wells Showing Observed Data and Model Results 


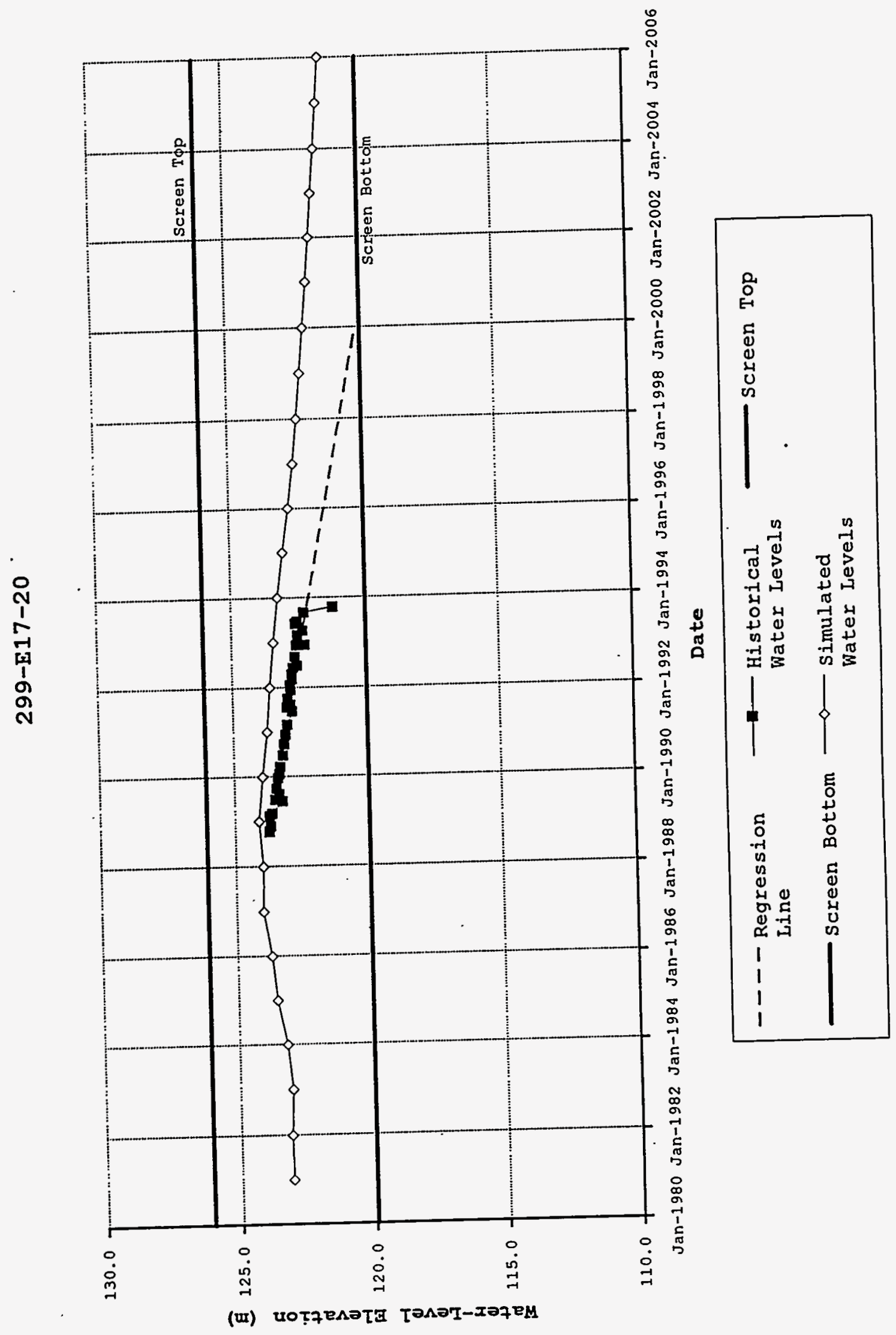

D.1 


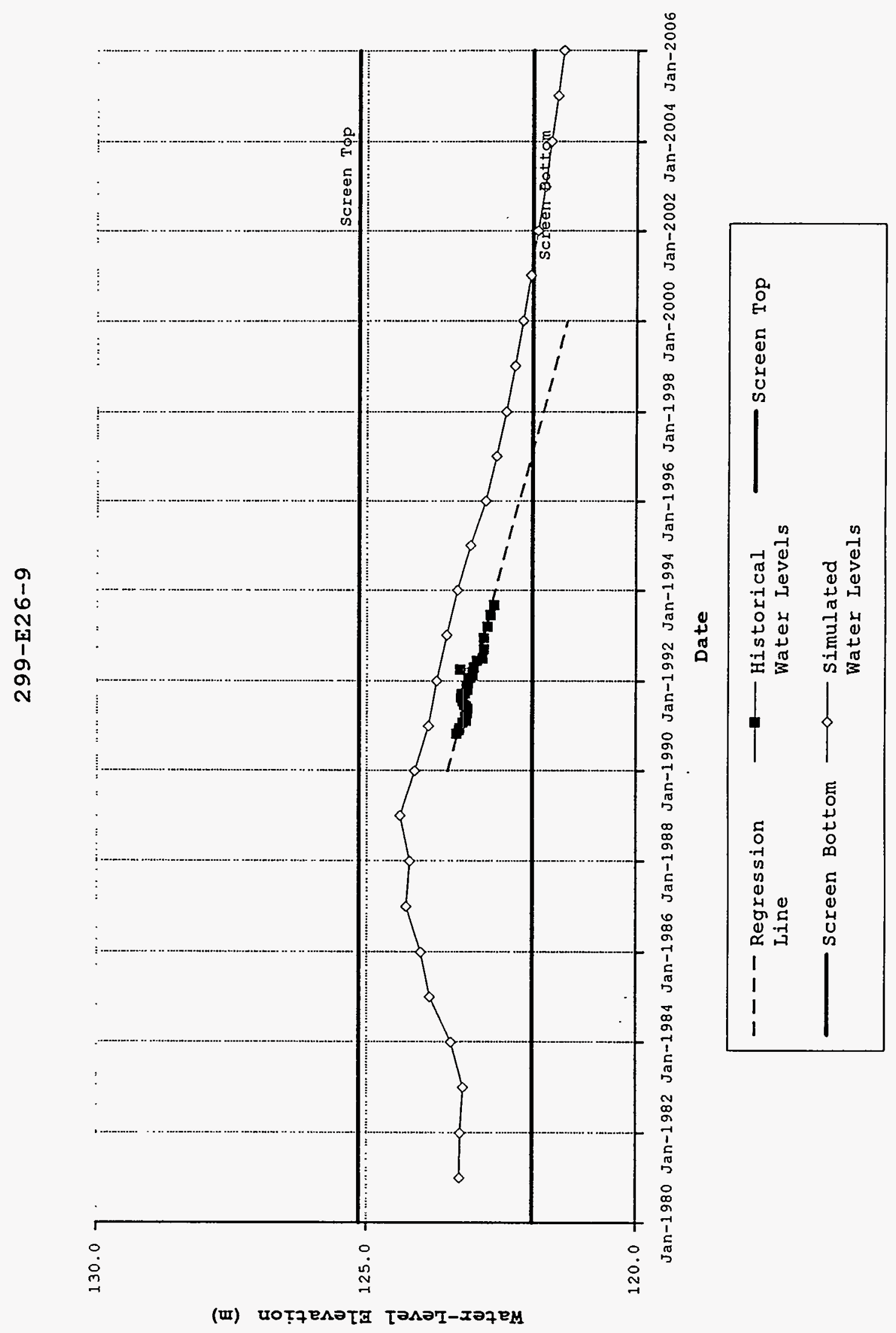

D. 2 


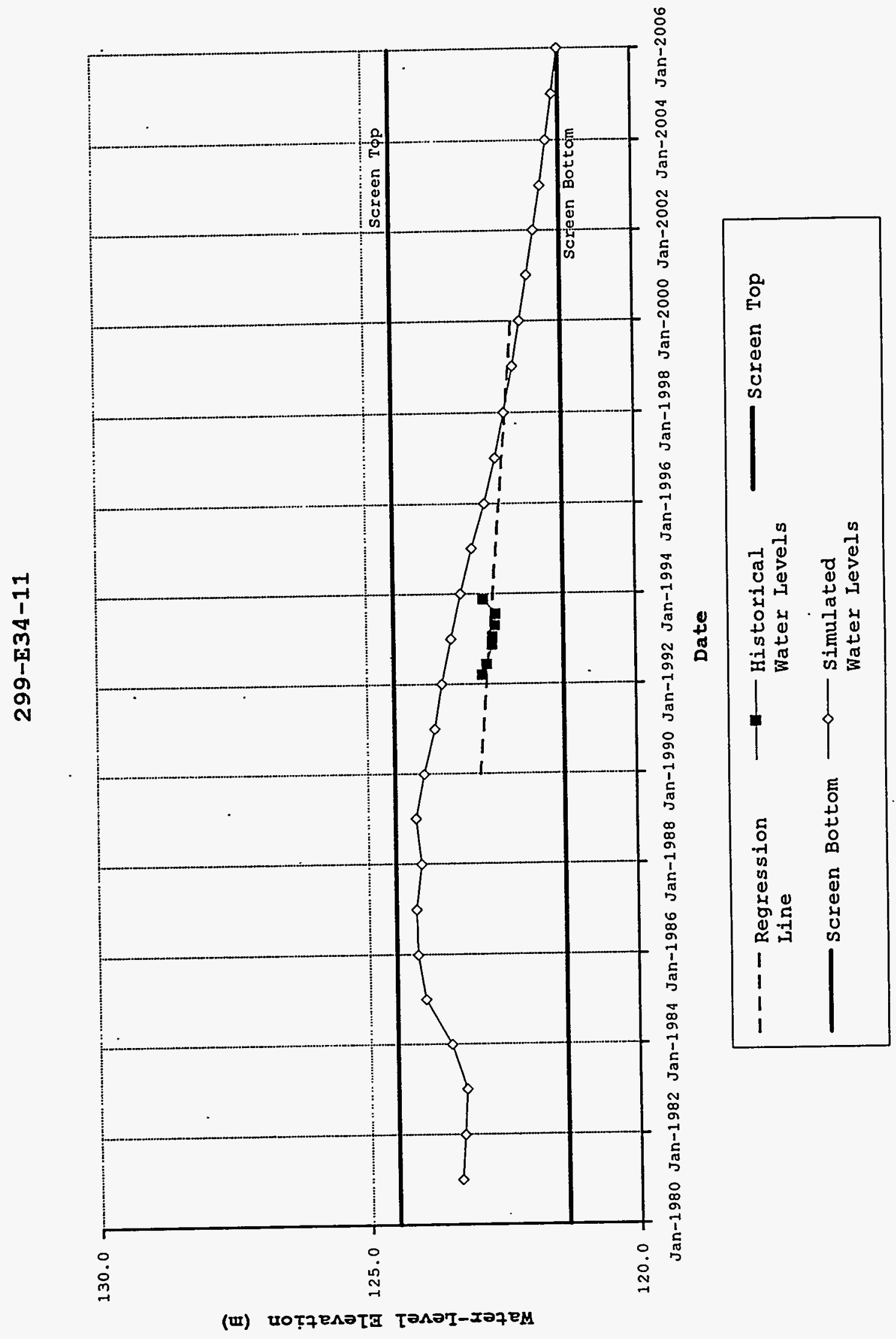

D. 3 


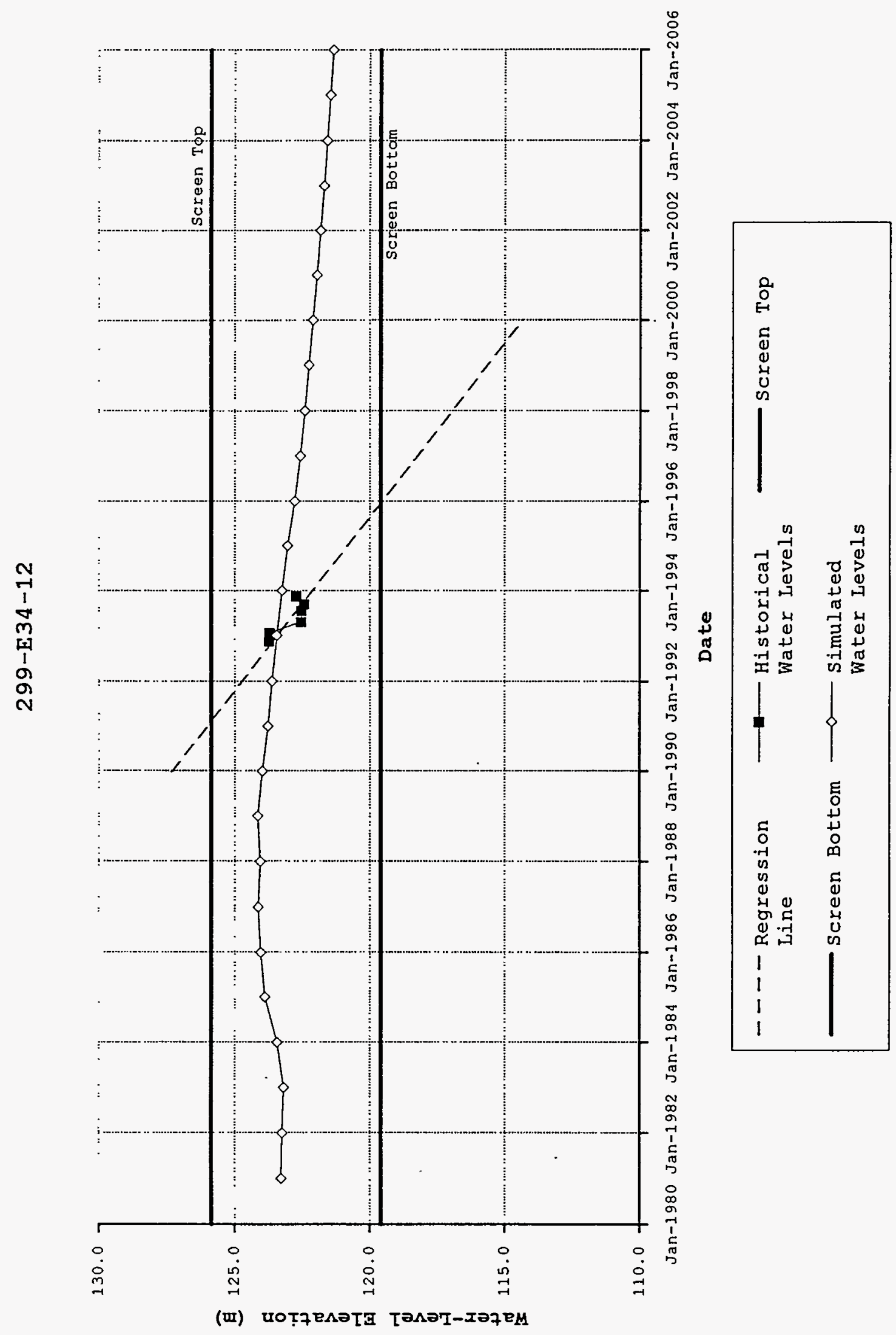

D. 4 


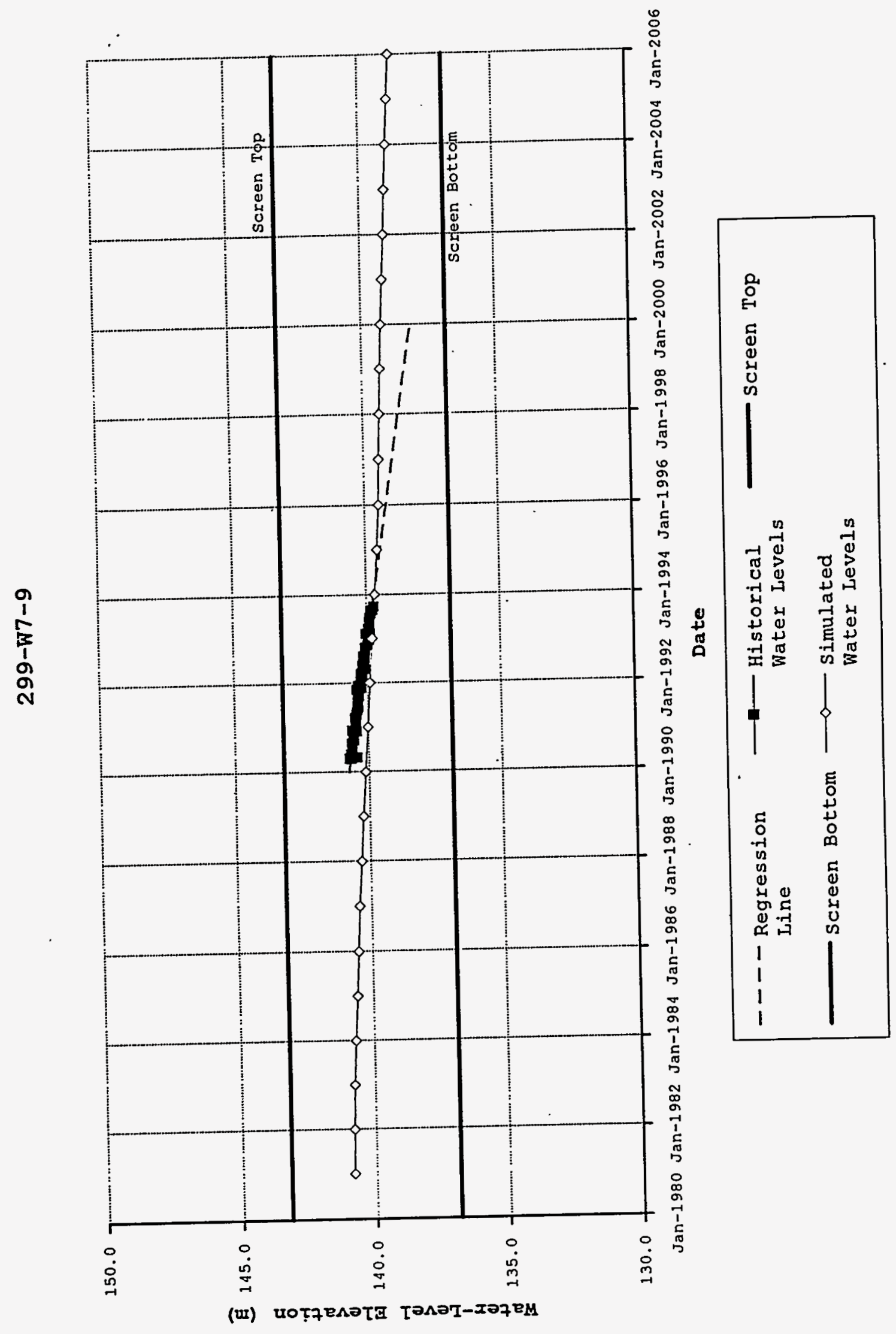

D. 5 


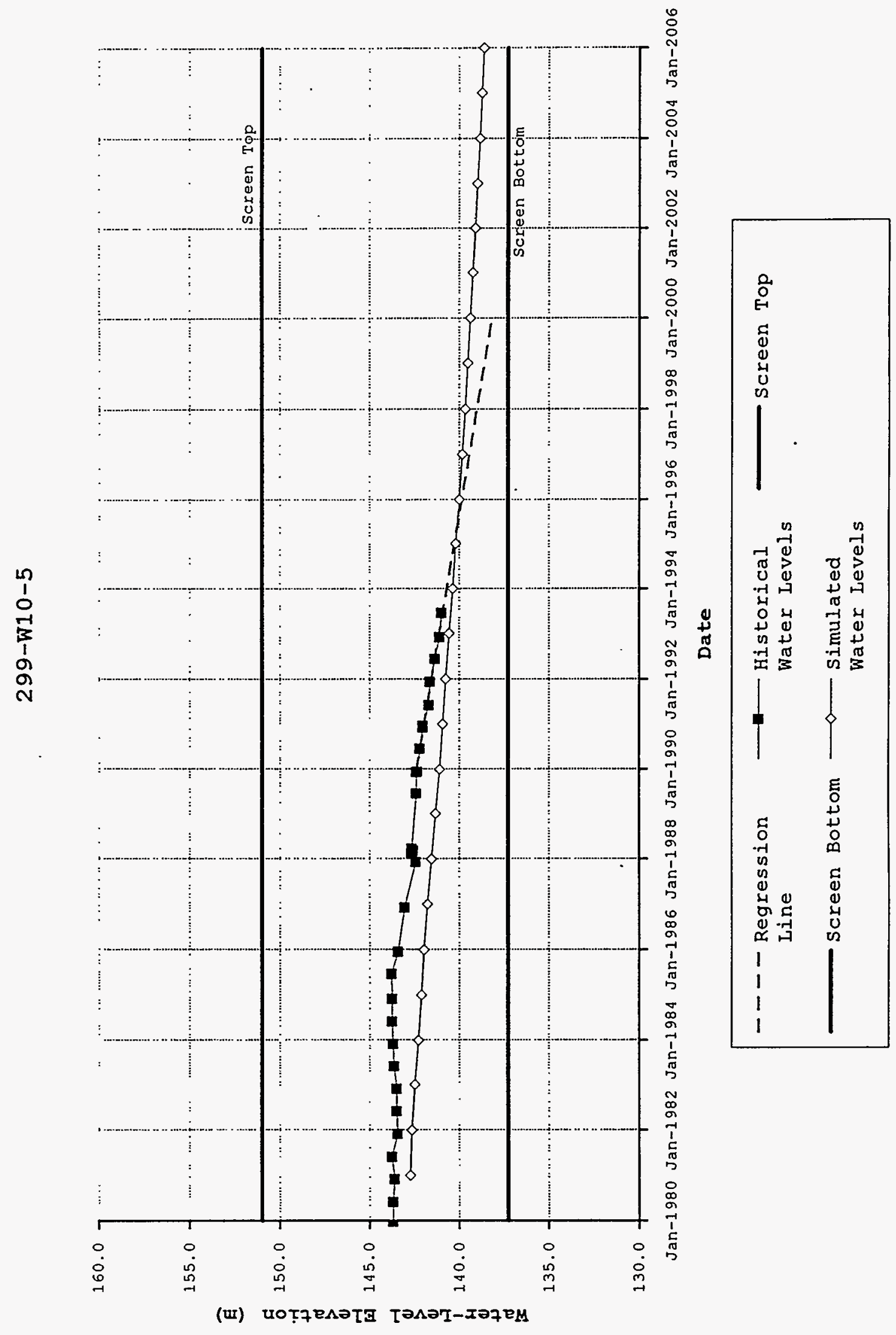

D. 6 


\section{9-พ18-32}

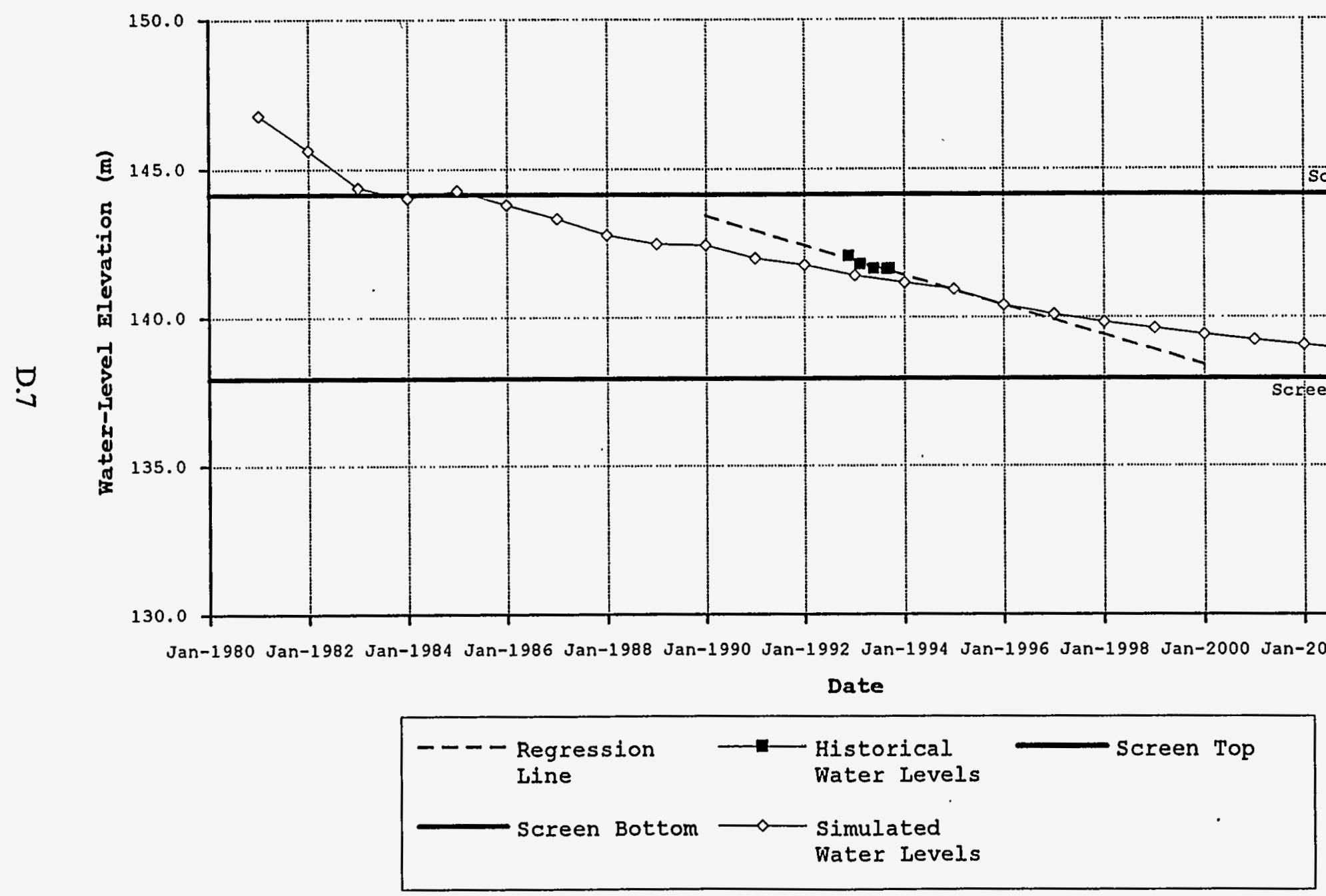




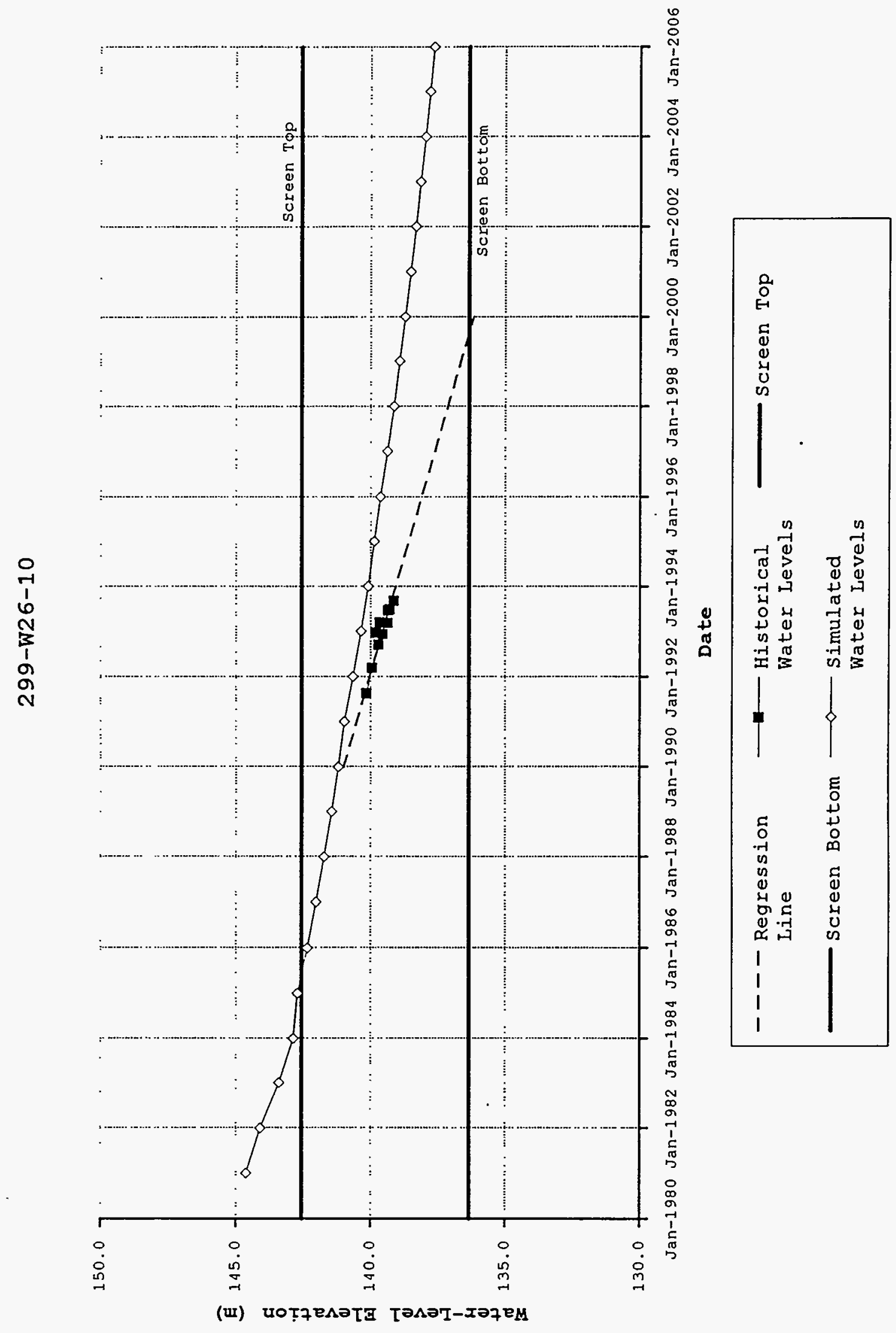

D. 8 


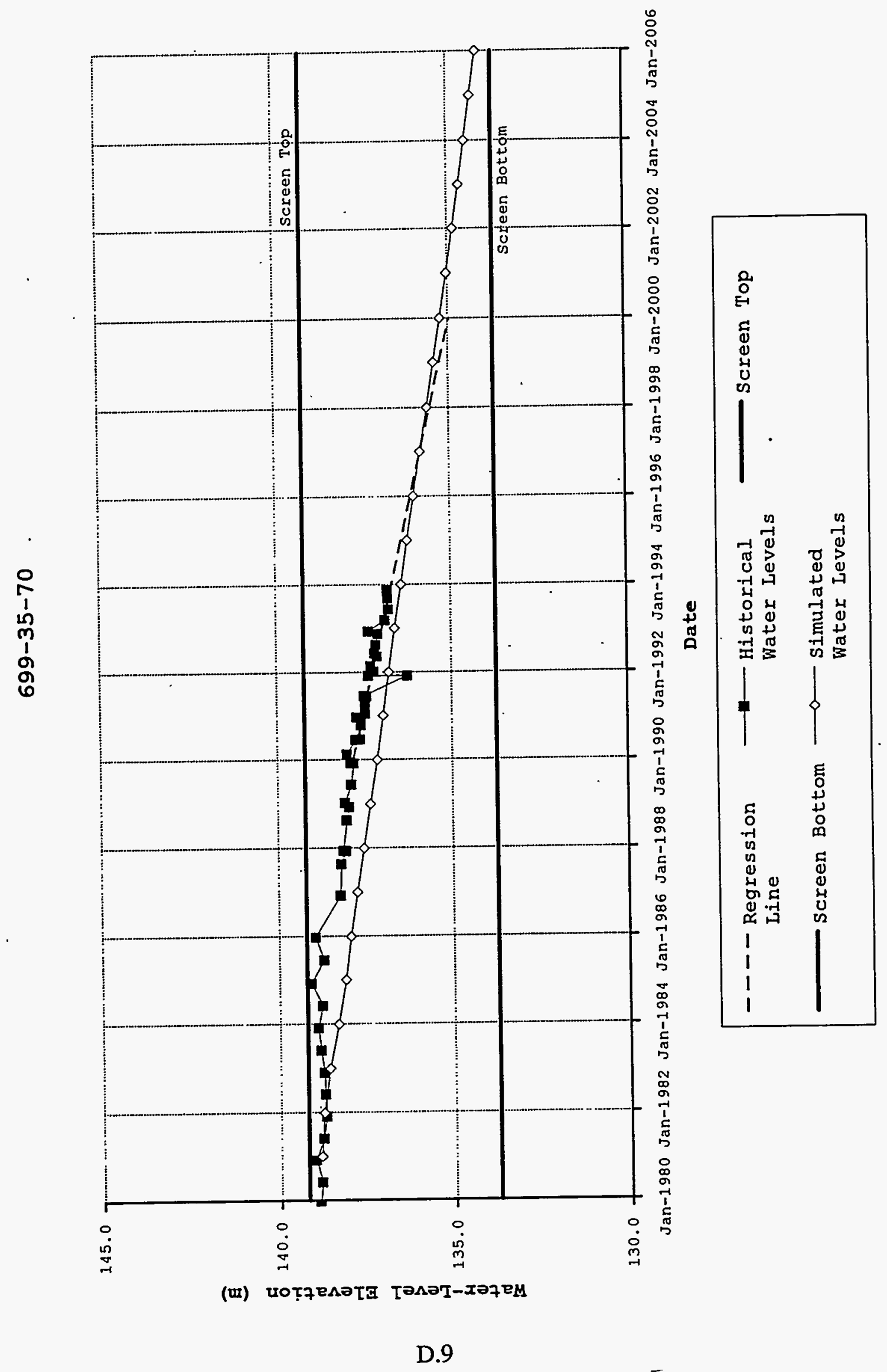




\section{Distribution}

No. of

Copies

OFFSITE

12 DOE/Office of Scientific and Technical Information

B. Blake

133 1st Avenue North

Minneapolis, MN 55401

R. Buck, Jr.

Wanapum Indian Band

P.O. Box 878

Ephrata, WA 98823

2 C. Cline

R. Stanley

Washington State Department of Ecology

99 South Sound Center

M.S. 7600

Olympia, WA $98504-7600$

R. A. Danielson

Washington State Department of Health

2 South 45th Ave.

Yakima, WA 98908

2 B. Drost

W. Lumm

U.S. Geological Survey

1201 Pacific Avenue, Suite 600

Tacoma, WA 98402

J. Erickson

Washington State Department of Health

Division of Radiation

Protection

Airdustrial Center

Building 5, M.S. L-13

Olympia, WA 98503
No. of

Conies

2 T. Gilmore

J. R. Wilkinson

Environmental Planning/

Rights Protection

Confederated Tribes of the

Umatilla Indian Reservation

Hanford Projects

P.O. Box 638

Pendleton, OR 97801

2 S. Harris

D. Powaukee

Environmental Restoration/

Waste Management

Nez Perce Tribe

P.O. Box 365

Lapwai, ID 83540-0365

2 R. Jim

B. A. Cook

Yakama Indian Nation

Environmental Restoration/

Waste Management

P.O. Box 151

Toppenish, WA 98948

R. Patt

Oregon State Department of

Water Resources

3850 Portland Road

Salem, OR 97310

\section{ONSITE}

\section{DOE Richland Operations Office}

J. D. Bauer

A3-42

G. M. Bell

A5-52

R. D. Freeberg

R. F. Brich

A5-19

M. J. Furman 
No. of

Copies

J. D. Goodenough

J. B. Hall

R. D. Hildebrand (10)

J. M. Hennig

R. G. Holt

R. A. Holten

R. G. McLeod

J. E. Mecca

P. M. Pak

R. M. Rosselli

W. A. Rutherford

C. O. Ruud

R. P. Saget

T. R. Sheridan

R. K. Stewart

K. M. Thompson

D. M. Wanek

12 Bechtel Hanford, Inc.

M. P. Connelly

K. R. Fecht

L. C. Hulstrom

G. L. Kasza

A. J. Knepp

M. J. Lauterbach

D. A. Myers

D. L. Parker

J. W. Roberts

L. C. Swanson

S. J. Trent

S. R. Weil

3 CH2MHill

J. V. Borghese

R. L. Jackson

R. E. Peterson

1 MACTEC

S. D. Barry

S7-73

3 U.S. Army Corps of Engineers

W. L. Greenwald

M. P. Johansen

W. D. Perro

H6-07

H4-80

H6-01

H6-04

H4-80

H6-01

H4-79

H6-02

H6-03

H6-03

H4-80

H4-80

H6-04

H6-04

H6-05

A5-19

A5-19
No. of

Copies

A5-19
A5-55
A5-55
S7-55
A5-15
R3-81
A5-19
R3-81
A5-19
K8-50
A5-58
S7-54
A5-54
S7-50
A5-19
A5-19
A5- 19

4 U.S. Environmental Protection Agency

P. R. Beaver

B5-01

D. A. Faulk

B5-01

D. R. Sherwood (2)

B5-01

4 Washington State Department of

Ecology

J. Atwood (3)

$\mathrm{N} 1-05$

D. N. Goswami

N1-05

6 Westinghouse Hanford Company

J. W. Cammann

H6-06

J. D. Davis

$\mathrm{HO}-33$

V. G. Johnson H6-06

A. G. Law H6-06

R. R. Thompson H6-32

J. S. Schmid

H6-06

\section{Pacific Northwest Laboratory}

M. P. Bergeron

K9-33

R. W. Bryce

K6-96

M. A. Chamness

K9-48

C. R. Cole

K9-36

J. L. Devary

P. E. Dresel

K9-56

J. C. Evans

K6-96

M. J. Fayer

M. D. Freshley

K6-96

K9-33

G. W. Gee

K9-36

T. J. Gilmore

K9-33

K9-48

S. H. Hall

R. E. Jaquish

K6-96

C. T. Kincaid

K9-25

K9-33

G. V. Last

T. L. Liikala

K9-48

K9-48

P. E. Long

K9-48

S. P. Luttrell (10)

K6-96

J. P. McDonald

Q. C. Macdonald

K6-96

P. D. Meyer

K6-96

D. R. Newcomer

K9-36

K6-96

K. B. Olsen

K6-96

J. T. Rieger

K6-96

M. L. Rockhold

K9-33 
No. of

Conies

R. E. Schrempf

F. A. Spane, Jr.

S. S. Teel

P. D. Thorne

V. R. Vermeul

W. D. Webber

S. K. Wurstner (10)

Publishing Coordination

Technical Report Files (5)

Public Reading Room
No. of

Copies

K6-86

K6-96

K9-48

K6-96

K6-96

K6-96

K9-36

K1-06
Routing

R. M. Ecker

M. J. Graham

P. M. Irving

S. A. Rawson

P. C. Hays (last)
SEQUIM

K9-38

K6-98

K6-81

K9-41

Distr.3 